University of Louisville

ThinkIR: The University of Louisville's Institutional Repository

Electronic Theses and Dissertations

$8-2020$

\title{
Aqueous redox flow batteries with Boron doped diamond as an electrode.
}

Alex M. Bates

University of Louisville

Follow this and additional works at: https://ir.library.louisville.edu/etd

Part of the Other Chemical Engineering Commons, Other Materials Science and Engineering Commons, and the Other Mechanical Engineering Commons

\section{Recommended Citation}

Bates, Alex M., "Aqueous redox flow batteries with Boron doped diamond as an electrode." (2020). Electronic Theses and Dissertations. Paper 3525.

https://doi.org/10.18297/etd/3525

This Doctoral Dissertation is brought to you for free and open access by ThinkIR: The University of Louisville's Institutional Repository. It has been accepted for inclusion in Electronic Theses and Dissertations by an authorized administrator of ThinkIR: The University of Louisville's Institutional Repository. This title appears here courtesy of the author, who has retained all other copyrights. For more information, please contact thinkir@louisville.edu. 


\title{
AQUEOUS REDOX FLOW BATTERIES WITH BORON DOPED DIAMOND AS AN ELECTRODE
}

By

Alex M. Bates

B.S., University of Louisville, 2014

M.E., University of Louisville, 2015

\begin{abstract}
A Dissertation
Submitted to the Faculty of the

J.B. Speed School of Engineering of the University of Louisville in Partial Fulfillment of the Requirements

for the Degree of
\end{abstract}

Doctor of Philosophy

in Mechanical Engineering

Department of Mechanical Engineering

University of Louisville

Louisville, Kentucky

August 2020 
Copyright 2020 by Alex Martin Bates

All rights reserved 



\title{
AQUEOUS REDOX FLOW BATTERIES WITH BORON DOPED DIAMOND AS AN ELECTRODE
}

\author{
By
}

\author{
Alex M. Bates \\ B.S., University of Louisville, 2014 \\ M.E., University of Louisville, 2015
}

A Dissertation Approved on

July 24, 2020

by the following Dissertation Committee:

\begin{tabular}{c}
\hline Dissertation Director \\
Dr. Sam Park \\
\\
\hline
\end{tabular}

Dr. Thomas Berfield

Dr. Hui Wang

Dr. Mahendra Sunkara

Dr. Gamini Sumanasekera 


\section{DEDICATION}

This dissertation is dedicated to my wife

Dr. Yukyung Kang

and my mother

Ms. Mary Karen Bates

who have always given me constant love, strength, and encouragement. 


\section{ACKNOWLEDGEMENTS}

First and foremost, I would like to take this opportunity to thank my advisor and mentor, Dr Sam Park, for his patience, guidance, encouragement, and belief in me as a researcher and as a person. I would also like to thank my committee members, Dr Mahendra Sunkara, Dr Gamini Sumanasekera, Dr Thomas Berfield, and Dr Hui Wang for their invaluable advice and numerous discussions which helped guide me along my path and improve my research. I am especially thankful to the Conn Center for Renewable Energy for allowing a graduate student the opportunity to lead and grow a redox flow battery lab from the ground up. This is a point of extreme pride and confidence that will be essential to my future as a researcher. A special thank you goes to Dr Hank Paxton and Dr Joshua Spurgeon who have been my hands-on, experimental guide and electrochemical guru, respectively. Lacking intimate knowledge and background in my research, both phenomenal researchers have nonetheless guided me to numerous successes. I would also like to recognize a list of particularly influential faculty, staff, and colleagues at the University of Louisville who, through numerous interactions and conversations, have helped advance my research and shape my ideologies of what a good researcher and person; Dr Jacek Jasinski, Dr Arjun Thapa, Dr Ellen Brehob, Dr Kevin Murphy, Dr Santanu Mukkherjee, Dr Daniel Jaramillo, Dr Muhammad Zain Akram, Dr Ruchia Dharmasena, Dr Patrick Ajayi, Dr Jacob Strain, Ms Sonia Calero-Barney, Mr Nicholas Schuppert, and Mr

John Jones. Last but, certainly not lost, my sincerest gratitude goes to my wife, Dr Yukyung Kang, and my family for years of encouragement and support. 해냈어! 


\begin{abstract}
AQUEOUS REDOX FLOW BATTERIES WITH BORON DOPED DIAMOND AS AN ELECTRODE
\end{abstract}

\author{
Alex M. Bates
}

July 24, 2020

As the interest and implementation of renewable energy accelerates, so does that of grid energy storage. It is widely believed that a cost-effective energy storage technology will bring about the proliferation of renewable energy. Redox flow battery (RFB) technology represents a promising solution to cost-effective grid energy storage. Compared to other technologies, RFBs have a long lifetime, high efficiency, are non-flammable, significantly reduce cost, and separately scale power and energy. The separation of power and energy enables increased energy capacity by simply adding electrolyte volume.

Of the challenges facing RFB technology, one readily apparent is the cost of the active species in the all-vanadium RFB, the most commercialized of the RFB iterations. One route aimed at answering this challenge is the examination of a wide range of lowcost active species. The aim of this dissertation is to extend that search through the utilization of an electrode material not previously considered for RFBs.

This dissertation will examine the utilization of boron doped diamond (BDD) as an alternative electrode in aqueous RFBs with the potential for a longer lifetime, higher efficiency, and lower cost active species compared to traditional RFB electrodes. The 
benefits of BDD include high conductivity, low capacitive currents, inertness, fouling and corrosion resistance, and high overpotential to gas evolution.

The growth of BDD using microwave plasma-assisted chemical vapor deposition is investigated using different growth recipes and substrates. Characterization includes scanning electron microscopy (SEM), energy-dispersive x-ray spectroscopy (EDX), Raman spectroscopy, and Fourier-transform infrared spectroscopy (FTIR). The viability of various redox chemistries is examined using electrochemical methods including charge/discharge cycling, cyclic voltammetry (CV), and electrochemical impedance spectroscopy (EIS).

It is found that $\mathrm{Ce}^{3+} / \mathrm{Ce}^{4+}$ and $\mathrm{Mn}^{2+} / \mathrm{Mn}^{3+}$ are promising redox couples on a BDD electrode. Based on peak-to-peak separations of $254.8 \mathrm{mV}$ for cerium and $140 \mathrm{mV}$ for $\mathrm{Mn}$, low overpotentials are evident. High reversibility and long lifetimes are apparent based on peak current ratios nearing unity and cycling data exceeding 300 cycles with improved peak current densities. In addition, the ability to scale up BDD was shown via growth on various materials including porous graphite and quartz fibers. 
TABLE OF CONTENTS

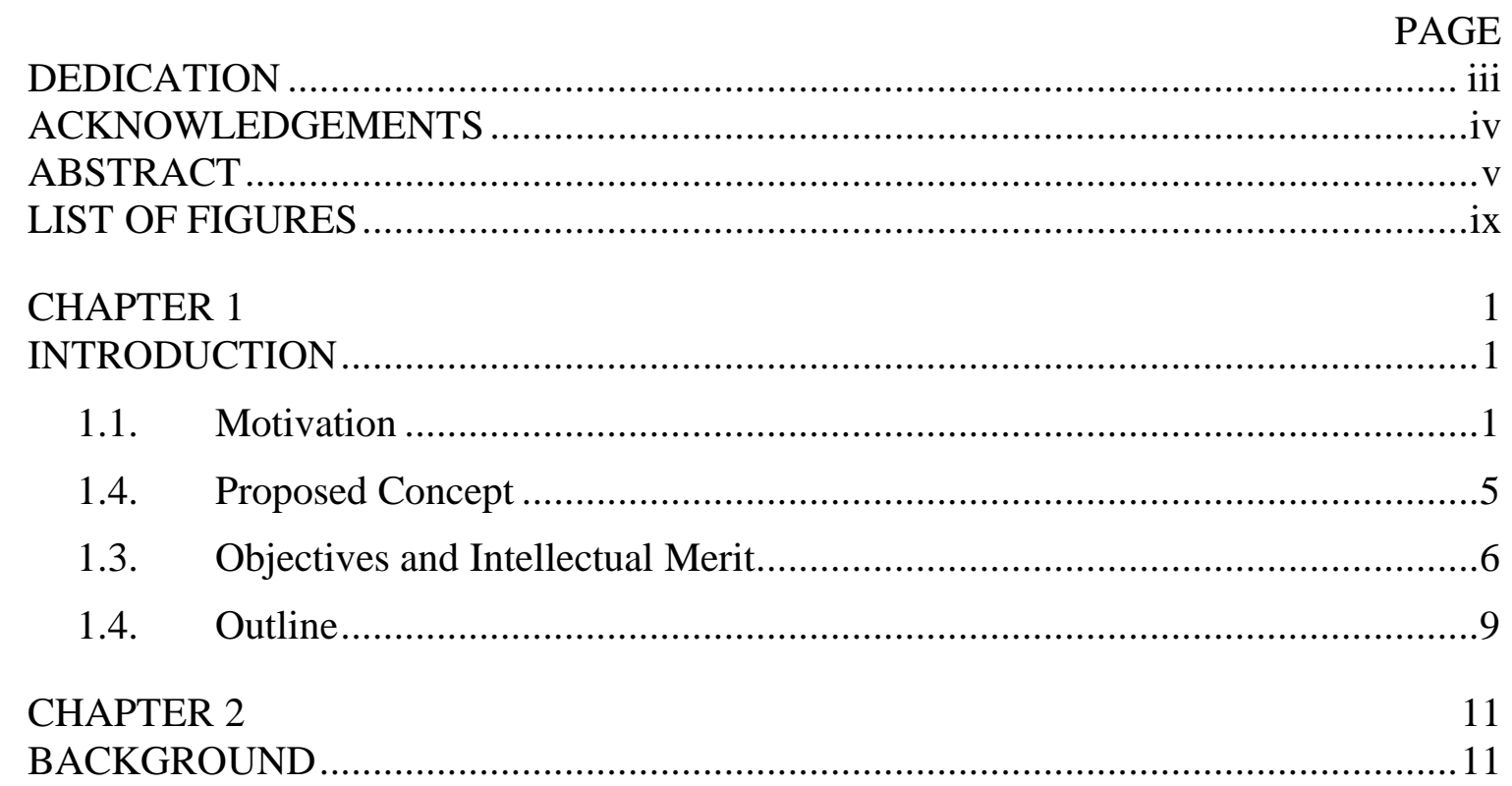

2.1. Redox Flow Batteries................................................................ 11

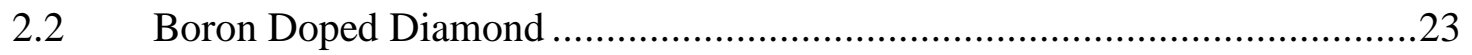

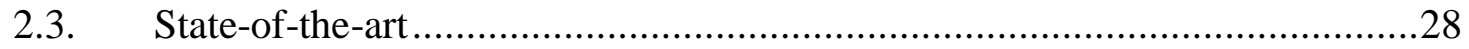

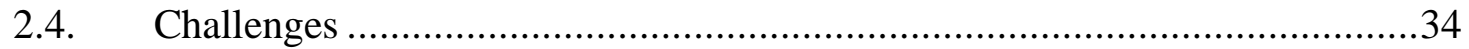

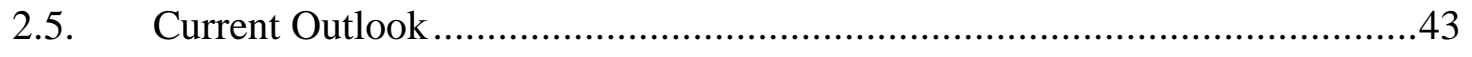

CHAPTER $3 \quad 48$

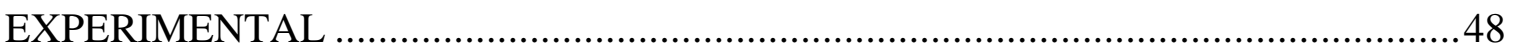

3.1. Electrochemical testing setups..................................................... 48

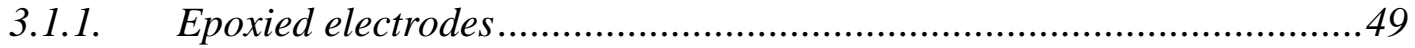

3.1.2. Multi-port boiling flask ................................................................50

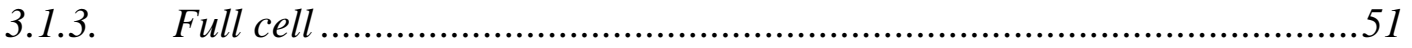

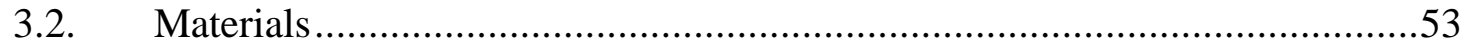

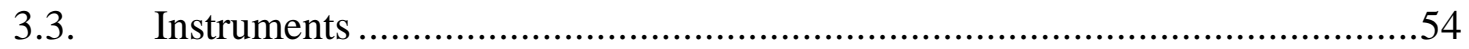

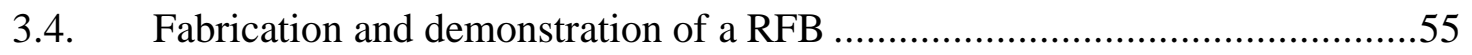




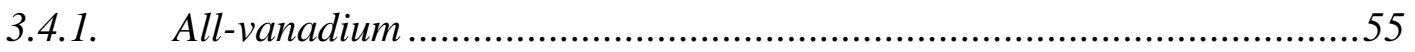

3.4.2. Vanadium/manganese …………………......................................63

3.4.3. Zinc/manganese ...........................................................................67

CHAPTER 4

THE $\mathrm{Mn}^{2+} / \mathrm{Mn}^{3+}$ AND Ce ${ }^{3+} / \mathrm{Ce}^{4+}$ REDOX COUPLES AS CATHOLYTES FOR RFBs 73

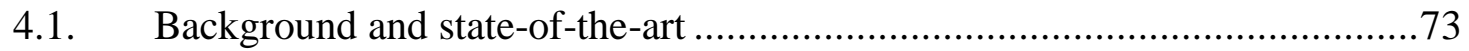

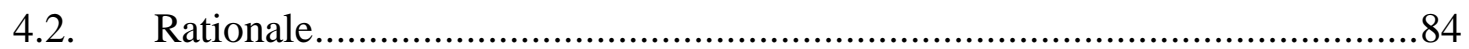

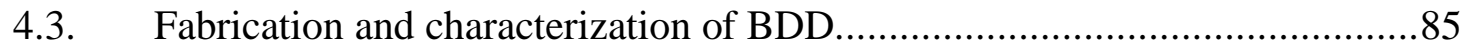

4.4. Performance of the $\mathrm{Mn}^{2+} / \mathrm{Mn}^{3+}$ and $\mathrm{Ce}^{3+} / \mathrm{Ce}^{4+}$ redox couples on BDD ..........95

4.5. Durability of BDD as an electrode in RFBs.............................................103

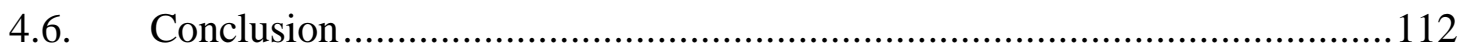

CHAPTER 5

EXPLORATION OF OTHER ECONOMICAL REDOX COUPLES ON A BDD

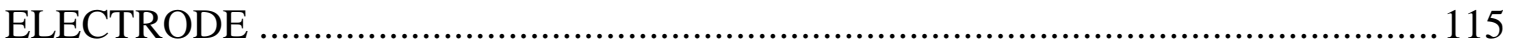

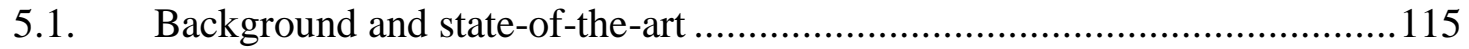

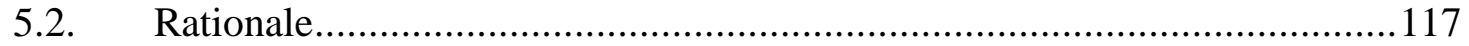

5.3. Testing of various redox couples on a BDD electrode …...........................118

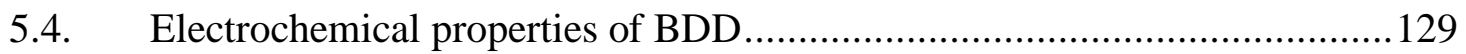

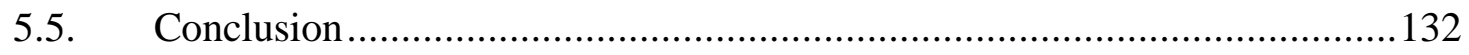

CHAPTER 6

SCALABILITY DEMONSTRATION OF BDD AS AN ELECTRODE FOR RFBS ... 134

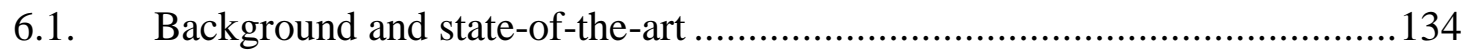

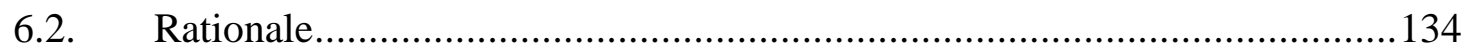

6.3. Utilize a full cell testing setup to demonstrate the capability of BDD .........135

6.4. Modify the BDD electrode to increase surface area ....................................137

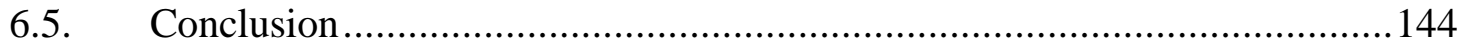

CHAPTER $7 \quad 145$

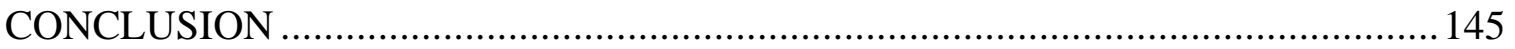

CHAPTER $8 \quad 148$

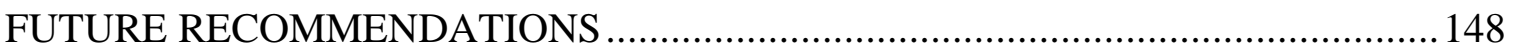

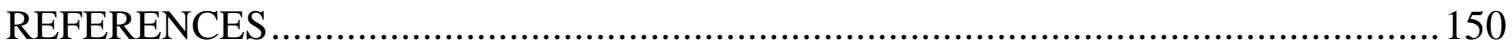

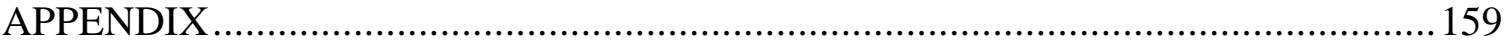

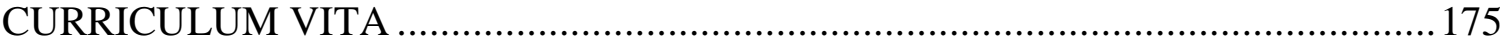




\section{LIST OF FIGURES}

FIGURE

PAGE

1. Schematic of a (a) pumped hydro system and (b) CAES system [25; 42] ...........14

2. Schematic of a (a) Li-ion battery and a (b) NaS battery $[39 ; 59] \ldots \ldots \ldots \ldots \ldots \ldots \ldots . . . .17$

3. Schematic of a RFB.

4. Effect of varying the boron content in BDD shown by (a) CV in $0.1 \mathrm{M}$ sulfuric acid with a scan rate of $100 \mathrm{mV} \mathrm{s}^{-1}$ and (b) $\mathrm{CV}$ in $1 \mathrm{mM} \mathrm{K}_{3}\left[\mathrm{Fe}(\mathrm{CN})_{6}\right]$ and $1 \mathrm{M}$

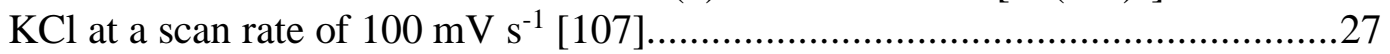

5. Component cost for a $1 \mathrm{MW}, 4 \mathrm{MWh}$ RFB of the listed chemistries [20]..........40

6. Example of an epoxied electrode. The active surface area on the left side is defined by the applied epoxy......

7. Multi-port boiling flask with 3 ports for reference electrode, counter electrode, and gas flow, 2 ports for filling of electrolyte solution, and one port for the working electrode.

8. Full RFB designed and fabricated at the University of Louisville. ....................52

9. Full RFB testing setup complete with two storage reservoirs and a peristaltic pump.

10. Initial charge curve to convert V(IV) to V(II).

11. Coulombic, voltage, and energy efficiency of the in-house fabricated flow battery which underwent 49 charge/discharge cycles.

12. Charge and discharge capacity over 49 cycles using the in-house fabricated allvanadium RFB.

13. UV-vis of the discharged anode electrolyte. expected to be $\mathrm{V}^{3+}$, compared to literature [12].

14. Five cycle $\mathrm{CV}$ of an unmodified graphite felt at a scan rate of $5 \mathrm{mV} \mathrm{s}^{-1}$ with $\mathrm{Ag} / \mathrm{AgCl}$ as the reference electrode.

15. Charge/discharge cycles for the RFB using vanadium as the anolyte and manganese as the catholyte.

16. Coulombic, voltage, and energy efficiency for an RFB using vanadium as the anolyte and manganese as the catholyte.

17. Charge/discharge cycles for an RFB using vanadium as the anolyte and manganese as the catholyte with a charge/discharge current density of $50 \mathrm{~mA} \mathrm{~cm}^{-}$ 2

18. Coulombic, voltage, and energy efficiency for an RFB using vanadium as the anolyte and manganese as the catholyte with a charge/discharge current density of $50 \mathrm{~mA} \mathrm{~cm}^{-2}$. 
19. Charge/discharge cycles for an RFB using zinc as the anolyte and manganese as the catholyte with a charge/discharge current density of $20 \mathrm{~mA} \mathrm{~cm}^{-2}$.

20. Coulombic, voltage, and energy efficiency for an RFB using zinc as the anolyte and manganese as the catholyte with a charge/discharge current density of $20 \mathrm{~mA}$ $\mathrm{cm}^{-2}$

21. SEM images of graphite felt (a) before testing, (b) after testing of the anode in the vanadium manganese RFB, (c) after testing of the cathode in the zinc/manganese RFB, and (d) higher magnification after testing of the cathode in the zinc/manganese RFB.

22. Morphology of $\mathrm{MnO}_{2}$ precipitates after charging to $90 \% \mathrm{SOC}$ in a $1 \mathrm{M}$ manganese and $3 \mathrm{M}$ sulfuric acid solution with (a) $0 \mathrm{M}$ titanium and (b) $1 \mathrm{M}$ titanium; (c) cyclic voltammetry of a $1 \mathrm{M}$ manganese and $3 \mathrm{M}$ sulfuric acid solution with a varying concentration of titanium (Ti $100 \%$ being $1 \mathrm{M}$ titanium) [51].

23. (a) Charge/discharge testing of a full RFB using a mixed titanium and manganese electrolyte [22]. (b) EIS of a symmetric cell [22].

24. (a) Linear polarization of a manganese electrolyte at different rotation rates [41].

(b) Limiting current at different potentials plotted against the square root of the angular velocity for a manganese electrolyte [41].....

25. Schematic and photograph of cylindrical cell used to operate a manganesehydrogen hybrid battery.

26. SEM image of a boron doped diamond surface showing continuous crystal growth across a silicon wafer surface.

27. SEM images of a BDD thin film on a tungsten substrate at (a) 5,000x,

(b) 10,000x, (c) 30,000x, and (d) 50,000x magnification.

28. SEM images showing the cross section of a BDD film on silicon substrate grown in the small Seki reactor with a microwave power of $1.2 \mathrm{~kW}$ and chamber pressure of 40 torr.

29. SEM images showing the (a) surface and (b) cross section of nitrogen incorporated diamond on a silicon substrate grown in the big Seki reactor with a microwave power of $1.6 \mathrm{~kW}$, chamber pressure of 60 torr, and stage temperature of $850{ }^{\circ} \mathrm{C}$.

30. BDD grown on a silicon substrate using diborane gas at (a) 5,000x, (b) 10,000x, (c) 30,000x, and (d) 50,000x.

31. Raman of a BDD thin film on a tungsten substrate showing the diamond, $\mathrm{sp}^{3}$ peak, at random positions on the diamond surface.

32. FTIR spectra of as-grown BDD.

33. $\mathrm{CV}$ of a BDD electrode at a scan rate of $3 \mathrm{mV} \mathrm{s}^{-1}$ in an electrolyte solution consisting of $0.1 \mathrm{M} \mathrm{MnSO}_{4}$ and $0.5 \mathrm{M} \mathrm{H}_{2} \mathrm{SO}_{4}$

34. $\mathrm{CV}$ scans of $10 \mathrm{mM} \mathrm{Mn}\left(\mathrm{NO}_{3}\right)_{2}$ in $1 \mathrm{M} \mathrm{HNO}_{3}$ at a scan rate of $1 \mathrm{mV} \mathrm{s}^{-1}$ before and after 180 cycles.

35. $\mathrm{CV}$ scans of BDD working electrodes in $1 \mathrm{mM} \mathrm{Ce}\left(\mathrm{NO}_{3}\right)_{3}$ in $1 \mathrm{M} \mathrm{H}_{2} \mathrm{SO}_{4}$ at scan rates from 1 to $5 \mathrm{mV} \mathrm{s}^{-1}$.

36. CVs of BDD working electrodes in a $50 \mathrm{mM} \mathrm{Ce}_{2}\left(\mathrm{CO}_{3}\right) 3,50 \mathrm{mM} \mathrm{Ce}\left(\mathrm{SO}_{4}\right)_{2}$, and $1 \mathrm{M} \mathrm{H}_{2} \mathrm{SO}_{4}$ electrolyte. 
37. CVs of a BDD working electrode in a $1 \mathrm{mM} \mathrm{Ce}\left(\mathrm{NO}_{3}\right)_{3}$ and $1 \mathrm{M} \mathrm{HNO}_{3}$ electrolyte. 100

38. $\mathrm{CV}$ of a BDD electrode at a scan rate of $3 \mathrm{mV} \mathrm{s}^{-1}$ in an electrolyte solution consisting of $0.1 \mathrm{M}$ manganese and $0.5 \mathrm{M}$ sulfuric acid. Two BDD substrates, silicon and tungsten, are compared along with platinum foil.

39. SEM and EDX of a BDD thin film on a silicon substrate after CV cycling in a manganese electrolyte. ( $a$ and $b$ ) were taken at a location with only diamond observable on the electrode surface. (c and d) were taken at a location with significant deposits of non-diamond material.

40. Raman of a BDD thin film on a silicon substrate.

41. Before and after images of fuel cell grade graphite showing a hole electrochemically etched through the graphite.

42. SEM images before and after long-term charging at high potential on BDD.....109

43. Raman spectra before and after long-term charging at high potential............... 110

44. $1 \mathrm{mM} \mathrm{Ce}\left(\mathrm{NO}_{3}\right)_{3}$ in $1 \mathrm{M} \mathrm{HNO}_{3}$ on fuel cell grade graphite and BDD compared using (a) cyclic voltammetry at $1 \mathrm{mV} \mathrm{s}^{-1}$, (b) potentiodynamic anodic polarization, and (c) cyclic voltammetry at different active species concentration.

45. CV scans of $100 \mathrm{mM} \mathrm{MnSO}_{4}$ in $500 \mathrm{mM} \mathrm{H}_{2} \mathrm{SO}_{4}$ on a BDD electrode.

46. CV of a BDD electrode at a scan rate of $20 \mathrm{mV} \mathrm{s}^{-1}$ in an electrolyte solution consisting of $0.1 \mathrm{M}$ potassium iodide. Two BDD samples are compared, one used and the other new, along with a graphite rod.

47. CV of a BDD electrode at a scan rate of $20 \mathrm{mV} \mathrm{s}^{-1}$ for platinum and $5 \mathrm{mV} \mathrm{s}^{-1}$ for the BDD electrode in an electrolyte solution consisting of $0.1 \mathrm{M}$ iron and $2 \mathrm{M}$ sulfuric acid. A BDD electrode is compare to platinum wire.

48. CV of a BDD electrode on a tungsten substrate at a scan rate of $20 \mathrm{mV} \mathrm{s}^{-1}$ in an electrolyte solution consisting of $0.1 \mathrm{M}$ copper and $2 \mathrm{M} \mathrm{H}_{2} \mathrm{SO}_{4}$.....

49. CV scans of $10 \mathrm{mM} \mathrm{CuSO}_{4}$ and $3 \mathrm{M} \mathrm{H}_{2} \mathrm{SO}_{4}$ on a BDD electrode.

50. CV of a BDD electrode on a tungsten substrate at a scan rate of $10 \mathrm{mV} \mathrm{s}^{-1}$ in an electrolyte solution consisting of $3 \mathrm{mM}$ ferricyanide and $1 \mathrm{M}$ potassium chloride.

51. CV scans of $1 \mathrm{mM} \mathrm{KI}$ and $1 \mathrm{M} \mathrm{KCl}$ on a BDD electrode.

52. $\mathrm{CV}$ scans of $1 \mathrm{mM} \mathrm{Fe}_{2}\left(\mathrm{SO}_{4}\right)_{3}$ and $1 \mathrm{M} \mathrm{H}_{2} \mathrm{SO}_{4}$ on a BDD electrode.

53. Solvent window comparison of BDD, glassy carbon, and platinum electrodes by $\mathrm{CV}$ in $0.5 \mathrm{M} \mathrm{H}_{2} \mathrm{SO}_{4}$.

54. Current density versus the square root of scan rate for $50 \mathrm{mM} \mathrm{Ce}_{2}\left(\mathrm{CO}_{3}\right)_{3}$ and $50 \mathrm{mM} \mathrm{Ce}\left(\mathrm{SO}_{4}\right)_{2}$ in $1 \mathrm{M} \mathrm{H}_{2} \mathrm{SO}_{4}$.

55. Evolution of fuel cell testing configurations using a (a) 2D BDD sample on a silicon substrate, (b) BDD grown on a porous graphite substrate with a CNC milled pocket or channels, and (c) BDD grown on a 2D but, porous graphite substrate.

56. BDD grown on a porous graphite substrate with a serpentine channel.

57. SEM images of BDD grown on a porous graphite plate showing growth of diamond on the top of the serpentine channels, inside the channels, and inside a pore in a channel. 
58. Growth of BDD on a porous graphite substrate using a high-pressure growth recipe.

59. BDD growth on quartz wool. The first image is quartz wool without any diamond growth. The middle and right images have significant diamond growth.............142

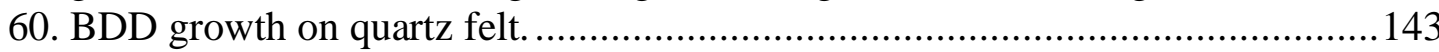

61. (a) Raman spectra of BDD grown on quartz felt and (b) $\mathrm{CV}$ of $1 \mathrm{mM} \mathrm{Ce}\left(\mathrm{NO}_{3}\right)_{3}$ in $2 \mathrm{M} \mathrm{HNO}_{3}$ at $1 \mathrm{mV} \mathrm{s}^{-1}$ on platinum wire and BDD on quartz felt. ....................143 


\section{CHAPTER 1 \\ INTRODUCTION}

\subsection{Motivation}

Due to the climate crisis, the trend in energy research worldwide is a movement from non-renewable to renewable energy sources. In contrast with non-renewable energy sources, which generate power on demand, renewables, such as wind and solar, are inherently intermittent. As a result, renewable energy sources require grid energy storage to store excess energy when generation exceeds demand, and make available energy when demand exceeds generation. Reduction in the cost of grid energy storage is necessary for the proliferation of renewable energy.

There exists a wide number of grid energy storage technologies competing for the prize of widespread commercialization. Currently, pumped-storage hydroelectricity commands $\sim 96 \%$ of the world's energy storage due to its maturity and longevity [37]. However, due to its extreme location dependence, it is not suitable for widespread use in terms of renewable energy. In fact, in locations prime for renewable energy, which have an absence of significant elevation change, pumped hydro falls flat.

Another serious competitor in recent years is the Li-ion battery. Spurred by advancements in the electric vehicle industry, Li-ion has received significant attention, along with increases in efficiency, reliability, and production methods. As a result, Li-ion for grid energy has begun rapid expansion into the category of grid energy storage. There 
are two primary downfalls to Li-ion technology, precious material costs and safety. Li-ion batteries require several precious materials that are speculated to increase significantly in cost in the coming years. With expansion into the grid energy storage domain, the increase in materials cost is likely to accelerate. Safety is a major concern due to the reactivity of the electrolyte with oxygen. Small discrepancies in manufactured materials can lead to defects in the 10 s of thousands of battery cells required for grid energy storage. Breakdown of one Li-ion cell can release a tremendous amount of heat and lead to a cascading effect with adjacent cells. This domino effect results in the rapid and catastrophic destruction of any cells in proximity. Due to the scale of grid energy storage, this is a significant issue that cannot be ignored.

Aside from the two grid energy storage technologies referred to previously, only one other has reached commercial success, the redox flow battery (RFB). RFBs are uniquely fit for grid energy storage of intermittent renewable energy. RFB technology offers the ability to separately scale power and energy, are very flexible in location requirements, can achieve high efficiency, and have demonstrated long cycle life. The major obstacle to the widespread adoption of the RFB is cost. Currently, the most promising iteration of RFB technology is the all-vanadium system, which has seen commercial success. A substantial portion of the capital cost for an all-vanadium RFB is the vanadium species itself. To overcome this obstacle, an alternative active species is required. Although many alternatives have been tried, all have failed for various reasons.

The goals outlined for grid energy storage by the U.S. Department of Energy include the metrics of capital cost, levelized cost, efficiency, and lifetime [38]. A redox flow battery meets these goals handedly in the areas of efficiency and lifetime. The all- 
vanadium RFB has significant ground to cover to reduce costs. The $\mathrm{Zn} / \mathrm{Br} \mathrm{RFB}$, another iteration of the RFB that has seen some commercial success, is closer at achieving the cost goals but falters a bit in the area of efficiency. The $\mathrm{Zn} / \mathrm{Br}$ RFB includes a plating reaction which suffers from the issues of dendrite formation, electrode shape change, and the recoupling of energy and power in a RFB system. For these reasons, the $\mathrm{Zn} / \mathrm{Br}$ system, which is far cheaper than the all-vanadium system, has not received as much commercial success.

The current state of the art in all-vanadium RFBs can achieve energy efficiencies greater than $95 \%$, with coulombic efficiencies greater than $99 \%$, at a current density of $25 \mathrm{~mA} \mathrm{~cm}^{-2}$ [35]. Even with increasing current density, which is desirable as it increasing the operating power density, a RFB can achieve an energy efficiency of $80 \%$ at $300 \mathrm{~mA} \mathrm{~cm}^{-2}$ [101]. The $\mathrm{Zn} / \mathrm{Br}$ system has demonstrated an energy efficiency of $82 \%$ at a current density of $40 \mathrm{~mA} \mathrm{~cm}{ }^{-2}$ [104]. BDD has never been examined for use in RFBs; however, it has received attention in a large number of other applications which marked success including, chemical sensors, reduction of $\mathrm{CO}_{2}$, and power systems, among many others $[49 ; 82]$.

How can an alternative, low-cost active species be employed in RFBs while maintaining the attractive benefits of the all-vanadium system? Pondering this question lead to consideration of the electrode material. In all RFBs, there exists a common theme, the utilization of various carbon allotropes including graphite, amorphous carbon, and carbon nanotubes, as electrode materials. To date, perhaps the most well-known allotrope of carbon, diamond, has not been considered for use in a RFB. The benefits of traditional carbon-based electrodes (conductivity, inertness, and durability) can be realized in 
diamond through boron doping. In addition, in an aqueous electrolyte, boron doped diamond (BDD) exhibits one of the widest solvent windows of any known electrode material.

The solvent window is one of the key features of BDD ripe for exploitation in realizing an alternative, low-cost active species. The solvent window is the potential separation between the onset of solvent breakdown. Solvent breakdown in an aqueous solution results in gas evolution, specifically oxygen and hydrogen gas. Gas evolution is fundamentally in kinetic competition with the redox reactions of the active species. This means, if gas evolution is occurring, the coulombic efficiency of the RFB is reduced. The wide solvent window of BDD extends the boundary at which gas evolution occurs in an aqueous solution. This boundary extension permits the inclusion of redox couples often seen as impractical on traditional carbon-based electrodes due. These redox couples, included by the advantages of BDD, may be low-cost and competitive electrochemically with existing technologies.

The other substantial benefit of BDD is its ability to withstand extreme potentials and highly acidic environments without exhibiting corrosion. Traditional carbon-based electrodes have been shown to corrode when under a high-potential environment. This corrosion coincides with the solvent window of the traditional carbon-based electrode due to the significant presence of oxygen at the cathode, which increases the rate of corrosion. Electrode corrosion results in a shortening of the RFB lifetime and may result in a short circuit of the RFB itself or leaking of the electrolyte solution. Leaking of the electrolyte could lead to loss of life or severe adverse effects on the surrounding environment. In addition, like gas evolution, corrosion is in kinetic competition with the active species 
redox reactions, leading to decreased efficiency. By allowing for the utilization of a high potential redox couple, without sacrificing efficiency or electrode integrity, BDD can catapult RFBs into the forefront of grid energy storage.

\subsection{Proposed Concept}

This dissertation seeks to expand the domain of redox chemistries that are of practical use in aqueous RFBs. By doing so, redox chemistries that are low-cost and high potential can be realized without limited coulombic efficiency or electrode degradation. This dissertation contains the demonstration and electrochemical characterization of two low-cost and high potential redox couples, $\mathrm{Ce}^{3+} / \mathrm{Ce}^{4+}$ and $\mathrm{Mn}^{2+} / \mathrm{Mn}^{3+}$, on a BDD electrode. The fabrication and characterization of BDD has been shown, along with material and electrochemical characterization of the BDD electrode itself. In addition, other redox chemistries have been explored, with various benefits, on BDD. In this manner, the viability of BDD as an electrode for RFB has been established. Last, an examination of what is necessary to bring BDD to full scale was conducted, including pathways to increased surface area.

The continuation of this research will primarily include extending this technology to full cell RFB testing. In doing so, the coulombic, voltage, and energy efficiencies can be obtained, allowing direct comparison to other RFB systems. This will require further refinement of the full electrochemical cell and a method of reliably increasing BDD surface area that does not result in significant non-diamond carbon content. Another important extension of this dissertation is the further examination of additional redox chemistries, potentially including expansion into non-aqueous chemistries. 


\subsection{Objectives and Intellectual Merit}

The goal of this research is to enable the development an aqueous redox flow battery (RFB) that is low cost, efficient, and has a long cycle life. To achieve that goal, this research will focus on the use of boron doped diamond (BDD) as an electrode in RFBs. BDD offers high conductivity, low capacitive currents, inertness, resistance to degradation, and high overpotential to gas evolution. BDD can enable access to cheap redox chemistries previously thought to be unviable due to low efficiency, corrosion, and/or decreased longevity. To date, BDD has not been explored as an electrode for use in RFBs. Research will be focused on fabrication, physical characterization, and electrochemical characterization to determine the viability of BDD thin films as an electrode in RFBs, with a focus on underexplored redox couples.

This research demonstrates the $\mathrm{Mn}^{2+} / \mathrm{Mn}^{3+}$ and $\mathrm{Ce}^{3+} / \mathrm{Ce}^{4+}$ redox couples using a BDD electrode which exhibits low overpotential to redox reactions, good kinetics, good reversibility, improved longevity, and a high overpotential to gas evolution. Manganese and cerium are earth abundant and low-cost compared to the already commercialized vanadium. Other potential redox couples, with promise due to low-cost, high energy density, and/or good system characteristics are explored.

This dissertation seeks to develop and examine BDD electrodes for use in a RFB. To achieve that aim, various objectives, shown below, have been determined. BDD electrodes will be grown under various conditions and characterized physically and electrochemically. Characterization will include morphology, boron doping concentration, non-diamond carbon (NDC) content, solvent window, reaction kinetics, and reversibility of RFB active species on BDD electrodes. Fouling and degradation resistance of BDD 
electrodes will be investigated as this is an important aspect of their cycle life. Several redox couples will be explored on the BDD electrode to determine their viability for use in a RFB. Redox couples to be tested will be chosen based on their bulk cost, electrochemical potential, novelty, and reversibility on BDD electrodes. Finally, this study will validate the scalability of BDD electrodes by demonstrating operation of full electrochemical cell using a BDD electrode in at least one of the half-cells. Methods to increase the surface area of BDD electrodes will also be explored as this is important to the scalability of BDD electrodes. These goals will be achieved through the competition of three objectives:

\section{Objective 1: The $\mathrm{Mn}^{2+} / \mathrm{Mn}^{3+}$ and $\mathrm{Ce}^{3+} / \mathrm{Ce}^{4+}$ redox couples as catholytes for RFBs Objective 2: Exploration of other economical redox couples on a BDD electrode Objective 3: Scalability demonstration of BDD as an electrode for RFBs}

The goal of Objective 1 is to demonstrate, through material and electrochemical characterization, the viability of a low-cost and high potential redox couple on a BDD electrode. The success of this objective will spur the continuing research of BDD as an electrode for RFBs. Objective 2 seeks to further develop the usefulness of BDD in a RFB setting by outlining additional redox couples, with other advantages, on a BDD electrode. In addition, this objective explores the surface electrochemistry of BDD. The next step in the development of BDD as an electrode for RFBs is showing a pathway to scaleup from a 2D electrode to a full scale RFB electrode with high surface area, Objective 3.

The realization of objectives in this study stands to explode the advancement of intermittent renewable energy by increasing profitability through low-cost, efficient, and long cycle life grid energy storage. In making renewable energy cost effective, a means for 
significantly reducing global emissions of greenhouse gases that are contributing to climate change is realized.

The impact and intellectual merit of this study may be summarized as follows:

1. Advancement in the field of BDD research focusing on thin film characteristics and their use in electrochemistry

2. Development of a novel RFB electrode that is resistant to gas evolution, fouling, and degradation in highly corrosive and extreme potential environments

3. Initiation of a new domain of possible advancements in the RFB research field

4. Proliferation of intermittent renewable energy by increasing profitability through low-cost, efficient, and long cycle life grid energy storage

Exploration of the viability of BDD as an electrode for RFBs will advance the knowledge of diamond thin film characteristics and their use in electrochemistry as applied to RFBs. Development of BDD as a novel RFB electrode will expand the domain of practical redox couples in aqueous RFB research by increasing energy efficiency through the reduction of gas evolution and increasing longevity through fouling and degradation resistance in highly corrosive and extreme potential environments. In doing so, this study will have a significant impact on the RFB research community, opening a new realm for possible advancements in the field in terms of decreased costs and increased longevity, power, and energy density. 


\subsection{Outline}

This dissertation will begin with a background of RFBs and BDD in chapter 2 followed by experimental methods in chapter 3. Chapter 4 covers the $\mathrm{Mn}^{2+} / \mathrm{Mn}^{3+}$ and $\mathrm{Ce}^{3+} / \mathrm{Ce}^{4+}$ redox couples along with the durability and fouling resistance of BDD. Chapter 5 demonstrates other potential redox couples and the electrochemical properties of BDD. Chapter 6 explores the scale up of BDD through increased surface area. Chapter 7 concludes the dissertation results and chapter 8 offers recommendations for future research.

A background to grid energy storage, RFBs, and BDD is provided in section 2.1 and 2.2. The current state-of-the-art in RFBs and BDD, along with their challenges, and the current outlook of the technology with be presented in section 2.3, 2.4, and 2.5, respectively. Section 3.1, 3.2, and 3.3 will present all experimental methods, including electrochemical cell designs, along with the materials and instruments utilized. Section 3.4 will describe the fabrication of a full RFB electrochemical cell followed by a demonstration of that cell with various chemical compositions, as part of a preliminary study.

In chapter 4 , the $\mathrm{Mn}^{2+} / \mathrm{Mn}^{3+}$ and $\mathrm{Ce}^{3+} / \mathrm{Ce}^{4+}$ redox couples are explored on BDD. Section 4.1 presents a background and the current state-of-the-are on these redox couples with respect to RFBs. Section 4.2 develops the reason for exploring these two redox couples in particular. Section 4.3 gives the method of fabrication for BDD and results from physical characterization. The electrochemical performance of the $\mathrm{Mn}^{2+} / \mathrm{Mn}^{3+}$ and $\mathrm{Ce}^{3+} / \mathrm{Ce}^{4+}$ redox couples on BDD is given in section 4.4. Section 4.5 discusses the durability and fouling resistance of BDD. The chapter is concluded in section 4.6.

Chapter 5 examines other possible redox couples on BDD. The chapter begins with a background and state-of-the-art in section 5.1 followed by the reason for the redox couple 
selections in section 5.2. Section 5.3 presents the results of electrochemical testing for the selected redox couples. Section 5.4 examines the electrochemical properties of BDD in an aqueous electrolyte solution. The chapter is concluded in section 5.5.

Chapter 6 looks at scale up of the BDD electrode to a full RFB electrochemical cell through increasing surface area beyond a 2D layer. Section 6.1 and 6.2 provide a background and rationale for scalability of BDD. Section 6.3 discusses the electrochemical cell requirements for demonstrating a full cell. Section 6.4 explores modifying BDD to increase surface area, through several techniques. The chapter is concluded in section 6.5. Chapter 7 offers a wrap up of the dissertation results. Chapter 8 looks to the future and offers results for continuing research. 


\section{CHAPTER 2}

\section{BACKGROUND}

\subsection{Redox Flow Batteries}

Traditional, non-renewable energy sources can meet demand by scaling the production of electricity. When demand is high, generation of electricity is increased through the consumption of more fuel (coal, natural gas, etc.). Renewable energy sources are inherently intermittent. The two most widespread renewable energy sources include wind and solar. Wind refers to the capture of wind energy using wind turbines connected to electrical generators. If the wind is not blowing, wind turbines cannot generate electricity. Solar refers to the capture of solar energy by conversion of sunlight into heat and then electricity or directly to electricity through photovoltaics. Like wind energy, solar energy can only be captured when sunlight is available. Due to the inherent intermittency of renewable energy sources, grid energy storage is required to complete a renewable energy system.

Grid energy storage refers to the large-scale storage of energy for use within a power grid. All grid energy storage devices consist of two major components, storage and power conversion. Grid energy storage is necessary for load balancing, the storage of energy when generation is in excess of demand and the release of energy when demand is in excess of generation. There exists a wide range of energy storage technologies, each with a different set of advantages and disadvantages. Currently, there is no clear winner in 
the competition for best energy storage device. As a result, grid energy storage research is widespread and fast paced. Energy storage is often separated into five broad categories: mechanical, chemical, electrochemical, electrical, and thermal. Some technologies can straddle two or more of these categories (e.g. electrochemical capacitors and thermochemical energy storage). Examples of grid energy storage technologies in each category are shown in Table 1.

Table 1. Categorization and examples of grid energy storage.

\begin{tabular}{c|l}
\hline \multirow{4}{*}{ Mechanical } & Pumped-storage hydroelectricity \\
\cline { 2 - 2 } & Compressed air energy storage \\
\cline { 2 - 2 } Chemical & Flywheel energy storage \\
\hline \multirow{4}{*}{ Electrochemical } & Gravitational potential energy storage \\
\cline { 2 - 2 } & Power to gas \\
\cline { 2 - 2 } & Hydrogen storage \\
\cline { 2 - 2 } & Redox flow battery \\
\cline { 2 - 2 } & Li-ion battery \\
\hline \multirow{4}{*}{ Electrical } & Sodium-ion battery \\
\cline { 2 - 2 } & Molten-state battery \\
\cline { 2 - 2 } Thermal & Superconducting magnetic energy storage \\
\cline { 2 - 2 } & Capacitors \\
\hline \multirow{2}{*}{} & Pumped heat electricity storage \\
\hline
\end{tabular}

The U.S. Department of Energy (DOE) released a set of goals for grid energy storage in 2013 which has been widely used as a source of comparison for current research endeavors. The near-term goals include a system capital cost under $250 \$ \mathrm{kWh}^{-1}$, a 
levelized cost of $0.20 \$ \mathrm{kWh}^{-1}$ cycle $^{-1}$, a system efficiency over $75 \%$, and a cycle life greater than 4,000 cycles [38]. For grid energy storage, a cycle is generally considered about one day. In this way, 4,000 cycles are roughly equivalent to a 10-year lifetime. A summary of various grid energy storage technologies and their characteristics is given in Table 2. The technologies presented in Table 2 represent the leading technologies based on maturity and current/theoretical cost. These technologies are briefly detailed in the following paragraphs to demonstrate the unique benefits and challenges present in grid energy storage research.

Table 2. Characteristics of grid energy storage technologies $[19 ; 37 ; 79 ; 90]$.

\begin{tabular}{ccccc}
\hline Technology & $\begin{array}{c}\text { Typical power rating } \\
(\mathrm{MW})\end{array}$ & $\begin{array}{c}\text { Lifetime } \\
(\text { years })\end{array}$ & $\begin{array}{c}\text { Cost } \\
\left(\$ \mathrm{kWh}^{-1}\right)\end{array}$ & $\begin{array}{c}\text { Efficiency } \\
(\%)\end{array}$ \\
\hline Pumped hydro & 500 & $30-60$ & $420-430$ & $75-82$ \\
CAES & 100 & $20-30$ & $60-125$ & $60-70$ \\
Li-ion battery & 10 & $5-20$ & $400-600$ & $85-95$ \\
Lead-acid battery & 10 & $3-4$ & $65-120$ & $70-80$ \\
NaS battery & 30 & $15-25$ & $520-550$ & $75-80$ \\
Vanadium flow battery & 50 & $20-30$ & $620-740$ & $80-90$ \\
Zn/Br flow battery & 50 & $20-30$ & $290-350$ & $70-80$ \\
\hline
\end{tabular}

Mechanical grid energy storage technologies viable for grid energy storage include pumped-storage hydroelectricity and compressed-air energy storage (CAES). Pumpedstorage hydroelectricity, or pumped hydro, stores energy by pumping a volume of water 
into a reservoir through a significant elevation change. A schematic of pumped hydro energy storage is shown in Figure 1(a). The reservoir of water represents a substantial amount of stored energy which is recovered by allowing the water to flow back down through turbines, converting the waters potential energy into electricity. Pumped hydro is the most widespread grid energy storage technology, accounting for $\sim 96 \%$ of the world's energy storage [37]. Pumped hydro is a mature technology with energy efficiency ranging from 70 to $85 \%$, a lifetime of $50-60$ years, and is currently the most cost effective despite its high capital cost $[37 ; 38]$.

CAES stores energy by compressing a volume of air using excess energy when generation exceeds demand. A schematic of a CAES system, where compressed air is stored underground, is shown in Figure 1(b). For grid energy storage, it is often proposed to store the compressed air in a large carven such as a natural or mined cave. CAES offers efficiency from 60 to $70 \%$, has a lifetime of 20 - 30 years, and low maintenance costs comparable to a simple cycle combustion engine [19]. Pumped hydro and CAES have very specific geographical requirements and so, are not in direct competition with each other.

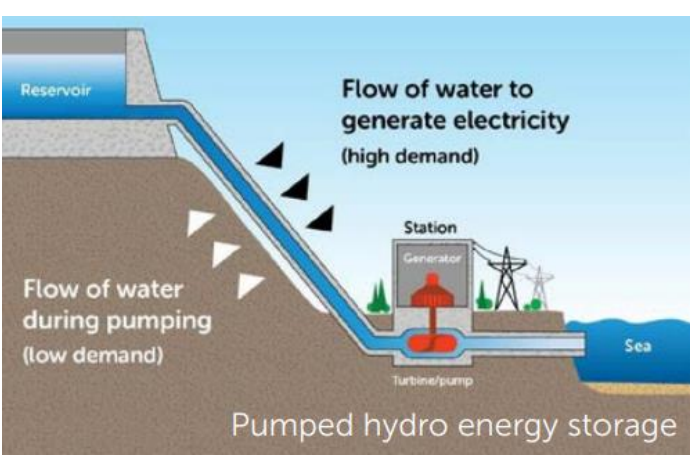

(a)

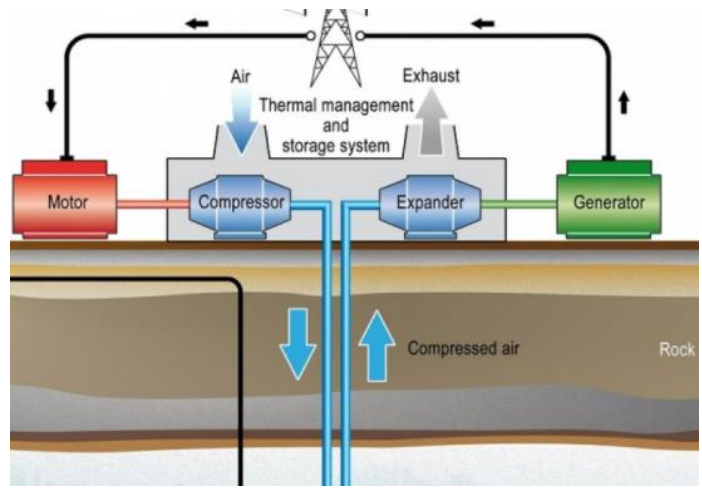

(b)

Figure 1. Schematic of a (a) pumped hydro system and (b) CAES system [25; 42]. 
Chemical energy storage is the storage of a fuel, in any phase, which contains potential energy in the form of chemical bonds. An example of a grid energy storage technology in this category is power-to-gas. Surplus energy is utilized to convert a low potential energy substance, such as water or carbon dioxide, into an energy rich substance, such as hydrogen gas or methane, respectively. The energy rich fuel is then stored using traditional methods until demand exceeds generation. In general, chemical energy storage allows for transportation and storage of fuel, with minimal energy loss long-term, utilizing existing infrastructure.

The category of electrochemical energy storage includes a wide variety of secondary batteries and is continuously expanding due to unceasing demand for applications ranging from cell phones to grid energy storage. Lithium-ion (Li-ion) batteries have recently attracted the most interest the area of grid energy storage as they are an established technology with advanced production methods driven by the electric vehicle industry. Li-ion batteries utilize Li ions as the main energy carrier. During charge and discharge, $\mathrm{Li}$ ions move through an electrolyte toward an electrode. Once at the electrode surface, $\mathrm{Li}$ ions proceed into the electrode via intercalation. Intercalation is the reversible incorporation of an ion or molecule into the layered structure of a material. A schematic of a Li-ion battery is shown in Figure 2(a) illustrating the intercalation of Li ions into the cathode during charge and anode during discharge. Li-ion batteries have a very high energy and power density compared to other grid energy storage methods which results in a much smaller system footprint. Li-ion batteries also exhibit good cyclability and high energy efficiency, greater than $85 \%$ [24; 79]. The lead-acid battery, perhaps one of the most widespread and well-known secondary battery, has been considered for grid energy storage 
due to its low capital cost and the maturity of the technology in terms of research and production [62]. In a lead-acid battery, hydrogen ions are the charge carriers, moving through an electrolyte solution. During charge, lead(II) sulfate is converted to solid lead at the anode and lead(IV) dioxide at the cathode.

For grid energy storage, another secondary battery type being heavily researched is the sodium-sulfur $(\mathrm{NaS})$ battery. This battery technology utilizes a molten salt as its electrolyte, transporting ions and containing the active species. Unlike Li-ion or lead-acid, the electrodes are liquid (sodium as the anode and sulfur as the cathode) and simultaneously act as the current collector and active species transport media. During discharge, a sodium atom donates an electron to the external circuit and the resulting sodium ion moves through the liquid sodium anode, across a separator, and into the liquid sulfur cathode resulting in a sodium tetrasulfide compound. In this type of battery, a separator is required to avoid self-discharge through reaction of the anode and cathode, while allowing for ion transport. A schematic of a NaS battery is shown in Figure 2(b). Beta alumina is the separator that only allows for the passage of $\mathrm{Na}^{+}$ions. The materials required for a $\mathrm{NaS}$ battery are inexpensive compared to $\mathrm{Li}$-ion. In addition, the $\mathrm{NaS}$ battery exhibits a long cycle life, high efficiency, and high energy density. 


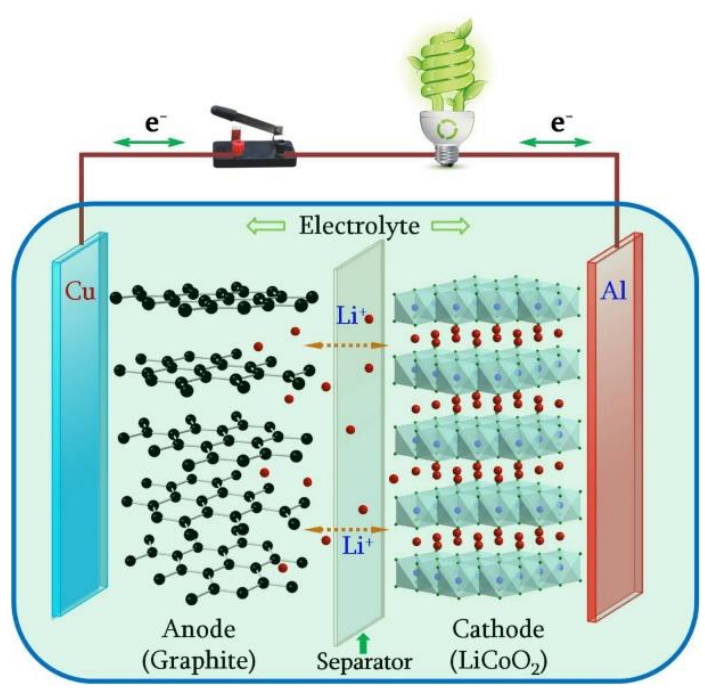

(a)

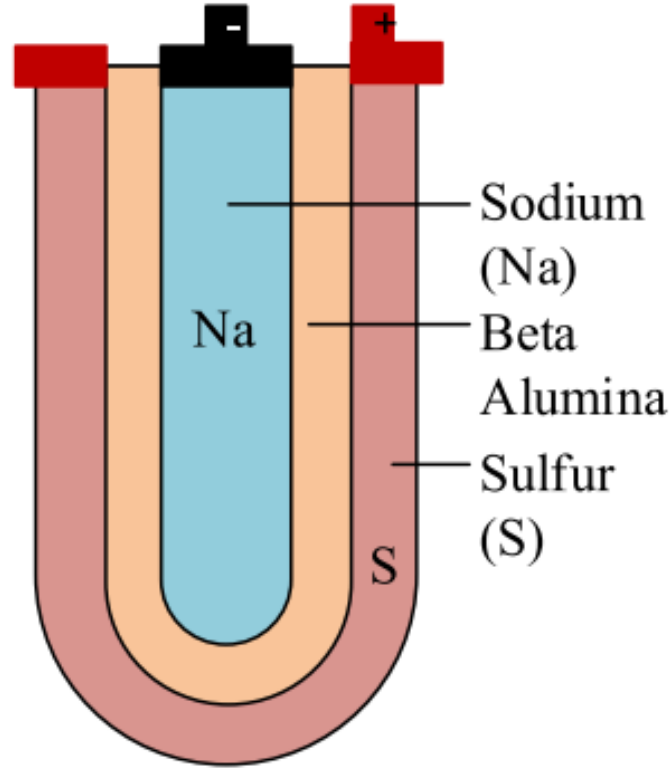

(b)

Figure 2. Schematic of a (a) Li-ion battery and a (b) NaS battery [39; 59].

The redox flow battery (RFB) is another type of electrochemical energy storage. In this technology, the active species is present in a liquid electrolyte that flows through the electrochemical cell. Both anode and cathode are stationary within the electrochemical cell and in most iterations, a separator is required. The flowing electrolyte supplies active species to the electrode to undergo electrochemical oxidation or reduction. In this way, the portion of the battery containing the potential energy, the liquid electrolyte, can be stored outside of the electrochemical cell itself. That is the key feature difference between RFB technology and other secondary batteries. The RFB is the focus of this dissertation and as such, will be detailed more thoroughly in the following chapter. A schematic of a RFB is shown in Figure 3. RFBs offer a long cycle life (>20 years), high efficiency ( $75-85 \%)$, no location dependence, and the ability to scale power and energy separately $[2 ; 80 ; 90 ; 108 ; 112]$. 
A RFB operates via an exchange of energy through the simultaneous electrochemical reduction and oxidation of redox couples in half-cells. A redox couple consists of an oxidizing species and its corresponding reducing species. For example, $\mathrm{V}^{5+}$ is an oxidizing species because it wants to accept an electron and $\mathrm{V}^{4+}$ is a reducing species because it wants to give up an electron. Together, $\mathrm{V}^{5+}$ and $\mathrm{V}^{4+}$ make up a redox couple. The fundamental RFB consists of two electrolytes (which contain two different redox couples), two electrodes, and an ion-exchange membrane, as shown in Figure 3. The electrolytes are stored in external tanks and are typically cycled through the electrochemical cell via pumps. The electrolyte with a redox couple that has a more positive potential compared to a reference is termed the catholyte. The electrolyte with a redox couple that has a less positive compared to the same reference is termed the anolyte. Additionally, by convention utilized in published literature, the side in which the catholyte flows contains the cathode (regardless of its function during charge and discharge). In the same manner, the side in which the anolyte flows contains the anode.

As the electrolytes flow, redox species are brought to the electrode surface where they will either give or receive an electron from an external source. During this process, the electrochemical cell is either charged or discharged and the converted species flow back to the storage tanks. The purpose of the ion-exchange membrane is to electrically separate the two half-cells while allowing preservation of charge balance through the migration of ions. The migration of active redox species would lead to self-discharge and a reduction in the efficiency and longevity of the cell. The ion-exchange membrane must block the transfer of the active species. Typically, preservation of charge balance is performed by a hydrogen ion, or hydron, which is provided by a supporting electrolyte such as $\mathrm{H}_{2} \mathrm{SO}_{4}$. The 
supporting electrolyte also supports the cells function by maintaining high ionic conductivity, $\mathrm{pH}$, and assisting in the prevention of active species crossover due to migration in the electric field.

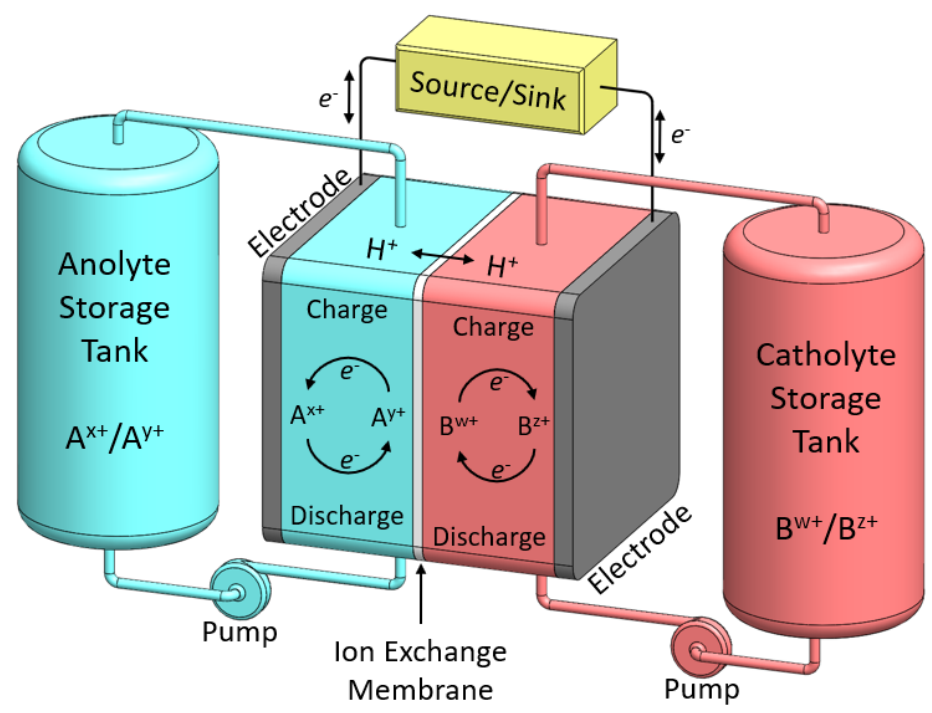

Figure 3. Schematic of a RFB.

The first recorded notion resembling the modern day RFB was found in a patent by Pierre André Pissoort, published in 1933 [77]. The next mention of the RFB, which describes the concept explicitly as it is thought of today, comes from a patent by Walther Kangro published in 1954 [52]. Following this was a publication in 1955 by Posner titled "Redox Fuel Cell" and a hybrid zinc-air flow battery with a flowing liquid electrolyte was tested in 1966 by Vertes et al. [78; 97]. In the 1970s, RFB research really began to take off with research by the National Aeronautics and Space Administration (NASA) in the USA, as well as groups in Japan and France $[6 ; 7 ; 8 ; 17 ; 31 ; 71 ; 93]$. In the 1980 s the, now commercialized, all-vanadium RFB by Maria Skyllas-Kazacos made its debut [88]. Today, 
the number of publications exploring different aspects of RFBs is ever increasing. There exists a wide variety of RFB types. RFBs can utilize different redox species, aqueous or non-aqueous electrolytes, plating reactions, gas phase electrolyte solutions, etc. The focus of this dissertation is on a wide variety of redox couples, configurations, material improvements, and optimizations.

As RFB technology has advanced and grown, categorizing different types of RFBs became necessary. To date, the best way to categorize RFBs is based on some key aspect of their operation instead of focusing on what redox couples they use. However, not all configurations fit neatly into a category and some fall completely outside the currently referenced categories. The three main categories separating RFB configurations are allliquid, hybrid, and, semi-solid.

In an all-liquid RFB, which includes the well-known all-vanadium RFB developed by Skyllas-Kazacos, both anolyte and catholyte remain in the liquid state during charge and discharge. For the vanadium example, vanadium ions transfer between the (II) and (III) oxidation states in the anolyte and the (IV) and (V) oxidation states in the catholyte; the ions remain in solution during the process. Some all-liquid RFBs employ a single chemical species, such as $\mathrm{V}$, as both the anolyte and catholyte active species, which avoids cross contamination due to crossover.

The hybrid RFB category includes configurations in which one or both electrolytes exist in any phase other than liquid. One or both electrolytes may undergo a plating reaction during charge or discharge so that a solid phase exists. In some embodiments of RFBs a gas phase exists in one half cell. In the hybrid RFB with deposition, one or both electrolytes are electroplated. Often the electrolyte undergoing electrodeposition is the anolyte during 
charge, while the catholyte remains a solution during charge and discharge. A wellresearched example of a hybrid RFB redox couple is zinc-bromine. The anolyte solution containing $\mathrm{Zn}^{2+}$ ions in its discharged state, undergoes electrodeposition at the anode to become solid zinc, $\mathrm{Zn}^{0}$. At the cathode, bromine remains as a solution.

The semi-solid category of RFBs is an emerging field of research with intriguing prospects. The semi-solid RFB, sometimes referred to as semiflow or slurry, uses a network of electrically conductive particles suspended in the anolyte and/or catholyte to act as the electrodes. When the concentration of particles is above some threshold, it forms an electrically conductive network allowing for the transfer of electrons from reaction to a current collector and out of the cell or vice versa. This slurry of electrically conductive particles can flow through the cell introducing fresh particles for reaction. With a redox couple that involves a plating chemistry, the cycling of particles through the cell is the key feature of the semi-solid RFB. The plating reaction can take place on the electrically conductive particles, or electrodes, and be removed from the cell; again, separating power and energy. So, systems that showed promise in safety and cost; but, where prohibited by a plating reaction, are now back on the playing field. Additionally, this flowing network of electrodes can offer increased reaction area compared to stationary electrodes.

The three previously described categories can be subdivided further by key aspects of their design. Often, the electrolyte solvent is categorized as aqueous or non-aqueous. Due to the low energy density of a RFB and the volume required for grid energy storage, this is an important categorization. Especially in recent years as research in non-aqueous RFBs have exploded primarily due to the Aziz group out of Harvard. Using water as a solvent is beneficial because it is cheap and accessible in large volumes. The drawback is 
the limited solvent window, outside of which the breakdown of water into hydrogen and oxygen occurs. Additionally, the solubility of some active species is limited in aqueous solutions. Non-aqueous solvents are, in general, significantly more expensive than aqueous solvents; however, they offer the ability to increase the solvent window and energy density well above that of an aqueous electrolyte RFB.

RFBs can also be categorizes by classifying the active species as non-organic or organic. Redox couples existing in non-organic active species, typically metals, tend to be cheaper to mass produce into electrolyte solutions and have a more facile reaction process. In fact, some redox couples react via outer sphere electron transfer in which the species is not required to adsorb onto the electrode surface for electron transfer to occur. This feature tends to result in increased kinetics and decreased overpotentials which leads to better energy efficiency. Organic active species are more complex and, in general, more expensive to produce with complex reaction steps. However, organic materials are tunable in that they can be "manufactured" to exhibit certain features such as high solubility, high reversibility, multi-electron transfer, etc.

Another commonly used categorization is based on the type of separator utilized, cation exchange membrane (CEM) or anion exchange membrane (AEM). RFBs utilizing CEMs must supply mobile cations to be transported through the separator, maintaining the charge balance in the electrochemical cell. CEMs are a well-researched technology due to their use in hydrogen/oxygen fuel cells. Unfortunately, they represent a large portion of the capital cost for RFBs. Recent research has been devoted to developing low cost CEMs but, they often come with a tradeoff of reduced energy efficiency and/or increased active species crossover. In recent years, AEMs have gained interest. With this type of membrane, 
an anion must be supplied in the electrolyte solution to balance charge. This is often a hydroxyl ion which results from a basic solution. AEMs offer increased resistance to active species crossover but, typically result in reduced conductivity, decreasing coulombic efficiency. Different variations of these separates have been utilized; however, they all operate using the same general principle of allowing the charge balancing species to cross.

Beyond these categories are some unique RFB configurations including the membraneless RFB. Membraneless RFBs were developed to remove the expensive ionexchange membrane from the cell and typically operate by utilizing a laminar flow regime so that minimal mixing occurs between the two redox couples; however, charge balancing can still move via migration. Another configuration utilizes a cheap separator filled with some form of ion exchange resin to facilitate charge balancing ion transfer. This method may result in significantly cheaper electrolyte separation; however, these separators also contain active species and the lack of a physical barrier may result in irreversible side reactions [36]. In addition to RFB, there are several types of flow batteries which store and release energy without using redox species. The concentration gradient flow battery stores and releases energy based on a concentration gradient, such as the separation and mixing of salt water [95]. A acid-base junction battery utilizes the energy involved in the separation of two solutions with a significant difference in $\mathrm{pH}$ levels [47].

\subsection{Boron Doped Diamond}

Carbon-based materials, such as glassy carbon, graphite, carbon nanotubes, etc., have been widely popular in the field of energy storage for years. These materials tend to be highly conductive, inert in a wide range of environments, and mechanically strong. In 
addition, some forms are carbon-based materials are relatively cheap and easy to fabricate into a wide variety of forms. In Li-ion batteries, graphite is universally employed as the anode material. During charge, Li ions intercalate in the graphite structure and are stored there until discharge. Graphite is beneficial due to its low voltage, reversibility, and mechanical strength during intercalation expansion. Carbon-based materials are also extensively utilized in RFBs. Although, intercalation does not occur in RFBs.

In RFBs, the electrode must be highly conductive, inert, mechanically strong, lowcost, and have a wide solvent window. The electrode must be highly conductive to facilitate the movement of electrons away from or toward the active species so that oxidation or reduction can occur, respectively. The electrode must be inert so that it does not interfere with the oxidation and reduction of the active species, creating unwanted side reactions which may degrade the integrity of the electrode. Mechanical strength is required so that the electrode can be fabricated into a structure with the ability to contain the liquid electrolyte. This typically entails significant pressure being applied to the structure itself. Stacks, which consist of multiple electrochemical cells, must have good electrical contact between each cell to allow for electron flow.

A key electrode feature required for RFBs is the solvent window of the electrode. The solvent window is the potential range between the onset of solvent breakdown at the anode and cathode. Every solvent has a potential at which the molecules making up the solvent breakdown. In aqueous solutions, with water as the solvent, breakdown consists of hydrogen evolution at the anode and oxygen evolution at the cathode. Based on the standard electrode potential, hydrogen evolution occurs at $0 \mathrm{~V}$ vs. SHE and oxygen evolution occurs at $1.23 \mathrm{~V}$ vs. SHE. These are the ideal values and are dependent on several 
factors including the $\mathrm{pH}$ of the solution, the contents of the solution, and the material at which reaction may occur.

In RFBs there is a balance between the electrochemical cells operating potential and the occurrence of gas evolution. The operating potential should be as high as possible while resulting in a minimum amount of gas evolution. In Li-ion batteries, gas evolution typically results in catastrophic failure of the battery because the seal, which prevents infiltration by atmospheric air, is compromised. RFBs can safely tolerate gas evolution but, it should be limited as it is an unwanted side reaction, leaching energy which could otherwise be stored, resulting in lower energy efficiencies. On the other hand, a higher potential electrochemical cell means a higher energy and power density which correlates with the cost of the energy storage system in terms of $\$ \mathrm{kWh}^{-1}$.

In RFBs, other electrode materials have been tested, typically metals, but, so far, nothing has rivaled the ability of traditional carbon-based materials. Many carbon-based materials have been utilized, each with a wide variety of modification; however, one allotrope of carbon that has not been considered is diamond. From here on, all carbonbased materials utilized in RFBs except for diamond will be referred to as "traditional" carbon-based materials. Diamond is a very intriguing material and, in recent years, has attracted increasing attention for applications in electronics and electrochemistry. To make diamond useful as an electrode, it must be conductive. There are several ways to modify diamond resulting in conductivity, mainly doping methods. The most often used dopant which results in conductivity for diamond is $\mathrm{B}$, which can substitutionally replace a $\mathrm{C}$ in the diamond lattice. $\mathrm{B}$ is to the left of $\mathrm{C}$ on the periodic table meaning it is deficient one electron, resulting in P-type conductivity in diamond. 
Currently, boron doped diamond (BDD) has not been considered as an electrode for use in RFBs; however, its application has been noticed for other electrochemical systems. The advantages of BDD include one of the widest solvent windows in aqueous solutions, low background and capacitive currents, reduced fouling compared to conventual electrodes, and the ability to withstand extreme potentials, corrosive, and high temperature/pressure environments [61]. For these reasons, BDD has gained popularity for electrochemical applications in the fields of electroanalysis, sensor technology, electrosynthesis, water treatment, and more [73]. Siuzdak et al. have utilized nanocrystalline BDD electrodes for the detection of the influenza virus [87]. He et al. explored the use of BDD electrode for the oxidation of organic pollutants to treat wastewater and remove toxins from drinking water [40]. Xu et al. are using BDD to reduce $\mathrm{CO}_{2}$ byproducts resulting from fossil fuel combustion into formic acid and hydrogen [107].

Of key interest to RFBs is BDD's wide electrochemical window, reduced fouling, and ability to withstand high potential and corrosive environments. The way BDD electrodes are fabricated has a significant effect on material properties including dopant density, non-diamond-carbon (NDC) content, grain morphology, and surface chemistry [61]. These features can in turn significantly affect the way the BDD electrode interacts with the electrolyte of a RFB. Boron content effects the electrical conductivity of diamond by acting as an acceptor, attracting electrons from neighboring bonds, creating a pathway for electrons to travel. With a high enough doping concentration, $>10^{20}$ atoms $\mathrm{cm}^{-3}$, BDD shows metal-like conductivity [46]. According to Macpherson, metal-like conduction in BDD exhibits a resistivity of $<10 \mathrm{~m} \Omega \mathrm{cm}$ [61]. Increasing the dopant concentration can lead to higher capacitance and the likelihood of NDC. So, an optimal doping concentration 
exists which gives high conductivity without significantly increasing NDC content. Hutton et al. found the optimum concentration to be $\sim 3 \times 10^{20} \mathrm{~B}$ atoms $\mathrm{cm}^{-3}$ [46]. Increased NDC content results in a more electrocatalytically active electrode which reduces the solvent window and increases the surface's susceptibility to fouling [61]. The changing solvent window of BDD due to changes in boron content are seen in Figure 4(a) where an increase in boron content results in a reduction of the solvent window. The effect of changes in boron content on the ability of the BDD electrode for the ferri/ferrocyanide redox couple is shown in Figure 4(b). An increase in boron content results in a lower peakto-peak potential separation which indications higher electrochemical reactivity.

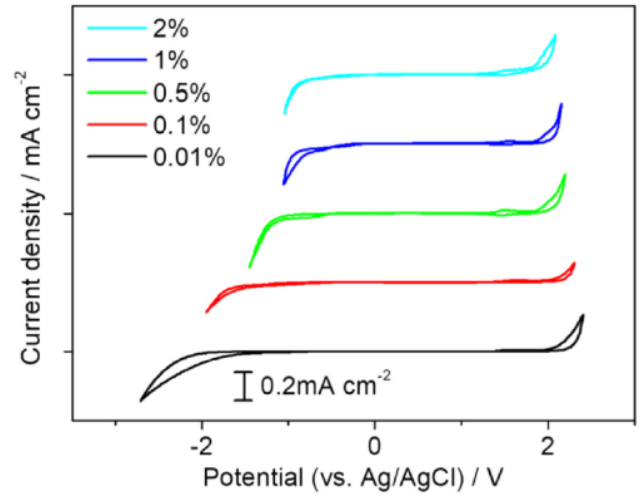

(a)

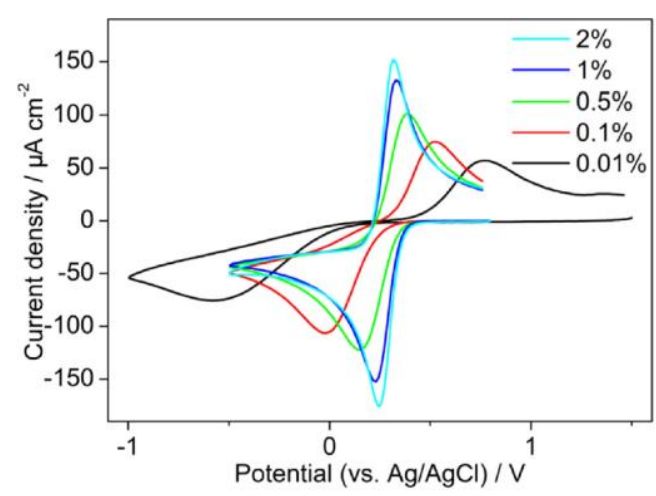

(b)

Figure 4. Effect of varying the boron content in BDD shown by (a) CV in $0.1 \mathrm{M}$ sulfuric acid with a scan rate of $100 \mathrm{mV} \mathrm{s}^{-1}$ and (b) $\mathrm{CV}$ in $1 \mathrm{mM} \mathrm{K}_{3}\left[\mathrm{Fe}(\mathrm{CN})_{6}\right]$ and $1 \mathrm{M} \mathrm{KCl}$ at a scan rate of $100 \mathrm{mV} \mathrm{s}^{-1}$ [107].

Surface termination of the BDD electrode has a strong influence on electron transfer kinetics and wetting properties [61]. Surface termination of BDD electrodes has been studied by many groups; however, it remains a source of contention. To date, a 
standard methodology does not exist for the determination of the surface functionality of diamond. This is due to several factors including the difficulty in determining the presence of hydrogen termination. Hydrogen surface termination causes semiconducting BDD to behave metal-like due to increased surface conductivity [46]. This behavior can make characterizing BDD difficult as a semi-conductive material can appear to be metal-like until the surface termination changes. The difficulties present in characterizing surface groups on BDD additionally influence the interpretation of results often creating confusing or contradictory findings amongst groups studying similar phenomena.

\subsection{State-of-the-art}

The current state of the art in RFB technology is the all-vanadium RFB which utilizes a vanadium redox couple in both electrolytes. The catholyte and anolyte redox couple chemical reactions and their standard electrode potentials are, respectively,

$$
\begin{array}{cc}
\mathrm{VO}_{2}^{+}+2 \mathrm{H}^{+}+e^{-} \leftrightarrow \mathrm{VO}^{2+}+\mathrm{H}_{2} \mathrm{O} & 0.991 \text { (vs. SHE) (1) } \\
\mathrm{V}^{3+}+e^{-} \leftrightarrow \mathrm{V}^{2+} & -0.255 \text { (vs. SHE) (2) }
\end{array}
$$

where the overall standard cell potential is $1.246 \mathrm{~V}$ [96]. In the literature, all-vanadium RFB energy efficiencies range from $80 \%$ up to $96 \%$ with varying current densities. Typically, energy efficiency goes down as current density goes up. This is an important point to take into consideration when examining RFB data. If the energy density is too low, the size of the electrochemical cell needed to produce a specific power output will be 
prohibitive. Gonzales et al. reported an energy efficiency of $95.8 \%$ at a current density of $25 \mathrm{~mA} \mathrm{~cm}^{-2}$ [35]. The reported energy efficiency is wonderful; however, the current density is quite low. Without factoring in overpotentials, pumping losses, or other sources of inefficiencies, an all-vanadium RFB system operating at $25 \mathrm{~mA} \mathrm{~cm}{ }^{-2}$ would require $3,210 \mathrm{~m}^{2}\left(34,552 \mathrm{ft}^{2}\right)$ of active area to generate $1 \mathrm{MW}$. At that current density, the capital cost of the electrochemical cell stack may become prohibitively expensive. Wei et al. achieved an energy efficiency of $80.1 \%$ at a current density of $300 \mathrm{~mA} \mathrm{~cm}^{-2}$ [101]. The results of Wei et al. show that more practical current densities are achievable while maintaining an energy efficiency comparable to pumped hydro [101].

The zinc/bromine flow battery (ZBFB) has had commercial success as well [53]. The ZBFB is considered a hybrid RFB due to the electroplating of zinc during charge. The ZBFB has a high energy density and low electrolyte cost; however, the electroplating of zinc means that energy and power are no longer separated, and the storage capacity is limited to the volume available for electroplating. Another drawback of the ZBFB is zinc dendrite formation, which can puncture the ion exchange membrane causing an electrical short and total malfunction of the RFB. Even so, ZBFB have been commercialized and are available for purchase from several companies. In the literature, the ZBFB has achieved energy efficiencies and charge/discharge current densities comparable to the all-vanadium RFB. Wu et al. reported an energy efficiency of $81.8 \%$ at a current density of $40 \mathrm{~mA} \mathrm{~cm}^{-2}$ with a maximum operational current density of $80 \mathrm{~mA} \mathrm{~cm}^{-2}$ [104]. Suresh et al. reported similar results with an energy efficiency of $79.4 \%$ at a current density of $40 \mathrm{~mA} \mathrm{~cm}{ }^{-2}$ [92]. Besides the all-vanadium and ZBFB, other RFBs have been commercialized with limited success. 
A slurry electrode was developed by Petek et al. utilizing Fe redox couples in both half-cells [76]. The Fe redox couple in the anode half-cell undergoes a plating reaction during charge. The slurry electrode allows for the charged, plated Fe species to flow out of the cell and be stored in tanks for later use, decoupling energy and power in a hybrid RFB. The slurry particles were multiwalled carbon nanotubes (MWCNTs) with a surface area of $40 \mathrm{~m}^{2} \mathrm{~g}^{-1}$. The MWCNT loading was 4.8 vol\%. The RFB was able to operate at current densities $>200 \mathrm{~mA} \mathrm{~cm}^{-2}$ [76]. This is a much higher current density then typically reported, even for the all-vanadium RFB. At this stage in development, reported voltage efficiency is quite low at about $50 \%$ [76]. This results in a low energy efficiency; however, the group believes significant improvements are possible in future iterations.

A wide variety of non-aqueous RFBs have been explored due to the ability to engineer wider solvent windows, faster electron-transfer kinetics, and operation in extended temperature ranges [45]. Non-aqueous RFBs can employ a wide variety of redox couples including organic molecules and metal-ligand complexes. Typical solvents employed in non-aqueous RFBs include propylene carbonate and acetonitrile, which has a solvent window of $5 \mathrm{~V}$ [45]. Like aqueous RFBs, non-aqueous RFBs require a supporting electrolyte. Typical supporting electrolytes include $\mathrm{TEABF}_{4}$ and $\mathrm{EMIPF}_{6}$ due to their solubility in organic solvents [45].

Ding et al. explored the use of ferrocene and cobaltocene as redox couples in a nonaqueous RFB with N,N-dimethylformamide (DMF) as the solvent [113]. This RFB exhibited a reaction rate up to $10^{-3} \mathrm{~cm} \mathrm{~s}^{-1}$, two orders of magnitude greater than the reaction rates found in aqueous RFBs [113]. The discharge potential was shown to be between 1.6 and $1.7 \mathrm{~V}, \sim 35 \%$ higher than that of an all-vanadium RFB [113]. The RFB developed by 
Ding et al. achieved an energy efficiency $85 \%$ at a current density of $1.5 \mathrm{~mA} \mathrm{~cm}^{-2}$ [113]. The cell potential and energy efficiency of this RFB meets the current standard in RFBs in general.

An all-iron non-aqueous RFB was with an open circuit voltage (OCV) of $1.34 \mathrm{~V}$ was proposed by Zhen et al. [115]. The solubility of the limiting redox couple was found to be $0.37 \mathrm{M}$ in the supporting electrolyte tetraethylammonium bis(trifluoromethylsulfonyl)imide (TEATFSI) [115]. The RFB showed good cyclability over 100 cycles with an average energy efficiency of $83.4 \%$ at $10 \mathrm{~mA} \mathrm{~cm} \mathrm{~cm}^{-2}$ [115]. This non-aqueous RFB demonstrates a cheap and abundant active species, Fe, with a high energy efficiency and OCV.

Organic redox couples have been increasingly explored in recent years fueled by the ability to tailor organic molecules to meet specific needs such as high solubility, fast kinetics, and high cell voltage. In addition, organic molecules tend to be composed of abundant, low-cost elements. Organic RFBs include those which utilize an organic species in one or both half-cells. The types of organic species can include organometallic materials and organic ligand complexes which can exist in aqueous and non-aqueous solvents. For these reasons, the number of possible electrolyte solutions consisting of one or more organic species is vast and rich for exploration.

An organic, quinone-based RFB using water as a solvent was demonstrated by Yang et al. [110]. As this RFB avoids expensive heavy metals and is water based, it has the potential to meet DOE cost targets while being non-flammable. Yang et al. were able to achieve an energy efficiency of $70 \%$ over 100 cycles with a current density of $100 \mathrm{~mA}$ $\mathrm{cm}^{-2}$; however, the power density was $55 \mathrm{~mW} \mathrm{~cm}^{-2}$ and the discharge potential was $<0.7 \mathrm{~V}$ 
[110]. Xing et al. demonstrated an all-organic RFB with a high theoretical solubility using acetonitrile as the solvent [106]. The organic redox couples showed an OCV of $2.89 \mathrm{~V}$ and achieved an energy efficiency of $66 \%$ at a current density of $7.5 \mathrm{~mA} \mathrm{~cm}{ }^{-2}$ [106]. The kinetics of the anolyte redox couple was found to be $6.92 \times 10^{-6} \mathrm{~cm}^{2} \mathrm{~s}^{-1}$ [106].

A membraneless RFB concept was presented by Gong et al. using the immiscible nature of an aqueous and non-aqueous electrolyte solutions [34]. In this RFB, the $\mathrm{Zn}^{2+} / \mathrm{Zn}$ redox couple in an aqueous electrolyte is used as the anolyte and a ferrocene $\left(\mathrm{Fc}^{+} / \mathrm{Fc}\right)$ redox couple in butyl acetate is use as the catholyte [34]. The $\mathrm{Zn}$ was supplied using a $\mathrm{ZnCl}_{2}$ salt, from which the $\mathrm{Cl}^{-}$ions become the charge balancing species and can cross the immiscible boundary via migration. This RFB demonstrated an energy efficiency of $51 \%$ at $0.1 \mathrm{~mA} \mathrm{~cm}^{-2}$ that was stable over 20 cycles [34].

As stated previously, BDD exhibits a wide solvent window, low capacitance, reduced fouling, mechanical strength, corrosion resistance in high temperature, pressure, and corrosive environments, and biocompatibility [61]. These features make BDD attractive for a wide variety of applications including power electronics and electrochemistry. A recently popular use for BDD in the field of electrochemistry as an electrochemical sensor due to its negligible adsorption phenomenon, low background current, and resistance to contamination [49]. Jevtić et al. utilized BDD to accurately determine the existence of bentazone, an herbicide with suspected toxicity in humans, at levels as low as $0.5 \mu \mathrm{M}$ [49]. The study determined BDD was an ideal sensor showing good selectivity without requiring electrochemical pretreatment or additives. Looking at published literature in recent years, a wide variety of electrochemical sensing applications have been found which utilize BDD well. 
Another intriguing application for $\mathrm{BDD}$ is the reduction of $\mathrm{CO}_{2}$ into a useful byproduct. $\mathrm{CO}_{2}$ is considered a greenhouse gas at high concentrations; however, it is the byproduct in most power plants and many industrial processes. The ability to control $\mathrm{CO}_{2}$ levels by conversion into a useful byproduct may prove vital to the welfare of human beings. A major challenge in $\mathrm{CO}_{2}$ reduction is product selectivity and competition with hydrogen evolution. Roy et al. demonstrated the selective reduction of $\mathrm{CO}_{2}$ into $\mathrm{CO}$ with minimal HER using a BDD electrode modified with $\mathrm{Cu}$ in an ionic liquid [82]. Other areas of interest using BDD electrodes include electro-organic synthesis, bio-sensor applications, electroanalysis, and nanoscale materials $[18 ; 58 ; 67 ; 99 ; 111]$.

BDD offers a wide solvent window in aqueous solutions, low capacitance, mechanical strength, high fouling resistance, and corrosion resistance. The wide solvent window allows for a higher potential electrochemical cell compared to traditional carbonbased electrodes while reducing the amount of gas evolution. This can lead to increased energy and power density, as well as increased energy efficiency.

In a RFB, low capacitance means a thinner double layer boundary and a lower potential barrier to reaction. The thinner double layer boundary allows for active species to diffuse closer to the electrode surface and the lower potential barrier requires less energy to force an electron from the active species to the electrode or vice versa. This results in higher voltage efficiency. High mechanical strength allows for assembly of stacks without destroying the electrode material. High fouling resistance is beneficial in some implementations of RFBs such as with the $\mathrm{Mn}^{2+} / \mathrm{Mn}^{3+}$ redox couple which can result in $\mathrm{MnO}_{2}$. Because of the high fouling resistance of $\mathrm{BDD}$, the $\mathrm{MnO}_{2}$ does not stick to and accumulate on the surface which would degrade the batteries performance. 
Corrosion resistance results in a battery with a long lifetime. Compared to traditional carbon-based electrodes, this is an important feature. When oxygen evolution occurs on traditional carbon-based electrodes, carbon corrosion tends to occur, reducing the lifetime of the material. In addition, as carbon corrosion occurs, this leads to increased oxygen evolution which, in turn, results in more corrosion. The strength of the diamond bond is such that it is highly resistant to carbon corrosion. Theoretically, a BDD electrode in a RFB can last forever. When the maintenance cost of a RFB is considered over its useful lifetime, the longevity of materials is very important. If an electrochemical cell can be built that seldom requires maintenance or replacement of its internal components, the projected cost over the lifetime of the battery is significantly reduced.

\subsection{Challenges}

The primary challenge with any grid energy storage technology boils down to cost. The current market leaders in renewable energy are intermittent by nature. This disadvantage in renewable energy, intermittency, is the major driver for grid energy storage. If the cost of renewable energy sources were cheap enough, perhaps an increased cost in grid energy storage could be offset. Unfortunately, this is not the current status of the field. Solar and wind technology are improving, leading to cost reductions; however, these technologies are relatively mature and so, require a significant technological breakthrough to significantly reduce costs. As it stands now, without subsidies, renewable energy is still not cost effective when compared to non-renewables. For these reasons, researchers have turned to grid energy storage as the source of cost reductions necessary to allow renewable energy to take command of the world's energy needs. If a sufficiently 
cheap and high efficiency grid energy storage technology is available, it can increase the effectiveness of renewable energy sources while reducing the overall cost of a renewable energy system.

Each energy storage technology has its own unique advantages and disadvantages when applied to grid scale energy storage. The disadvantages of pumped-storage hydroelectricity include a large capital cost, location dependence, low energy density, and environmental concerns $[11 ; 13 ; 60]$. Pumped hydro has a low energy density so, a pumped hydro system is large and requires a significant initial capital investment and a long timeframe to develop and build. Pumped hydro also requires very specific geological features to be present, namely a large elevation change near a water source with a proper climate. Building a manmade reservoir with the proper elevation difference is cost prohibitive. If the water in the reservoir turns to ice and/or evaporates, that results in a loss of stored energy. Also, pumped hydro stations can have a significant impact on the surrounding wildlife by adversely affecting their natural habitat. Even though pumped hydro represents the most cost effective and mature grid energy storage technology, the disadvantages make it unusable in a vast majority of locations around the globe.

CAES is a promising technology in terms of cost and efficiency; however, it suffers from a similar location dependence as pumped hydro. There must be a large cavern present into much a pressure gradient can be established. These caverns can result from manmade activities but, building a cavern for this specific use is cost prohibitive without the benefit of mining a sellable product. In that regard, the development and building of a grid energy storage CAES system is very long-term. Interest in above ground CAES systems has grown in recent years, unfortunately when applied to grid energy storage, the cost of vessels to 
store pressurized gas become prohibitive. In addition, CAES systems significantly alter the habitat in which they are developed which may have a profound effect on the local wildlife.

Li-ion has received more attention in recent years than any other energy storage technology due to the advancement of electric vehicles. Li-ion does not have the location dependence concerns of pumped hydro and CAES; however, there are environmental concerns due to the flammability of the Li-ion batteries themselves. Li-ion batteries have had catastrophic failures in cell phones, e-cigarettes, and electric vehicles. On a grid energy storage scale, the result could be even more devastating. Currently, Li-ion technology is relatively cost effective and so, has been deployed in several locations for grid energy storage. This is a result of the maturity of the technology and significant push by Tesla in the fabrication of Li-ion batteries with its Giga facility. Unfortunately, current iterations of Li-ion batteries require materials that are not abundant, including the Li itself. As the number of electric vehicles continue to grow and if the technology advances in the grid energy storage field, material costs will only increase. However, concerns over the availability for cost-effective lithium (among other required rare materials) of sufficient quality and the safety of the Li-ion battery in a large grid scale structure, strongly point to the need for alternative solutions $[54 ; 66 ; 81]$. Since there is little left to gain in terms of efficiency, this technology appears to some to be a dead end in terms of grid energy storage. $\mathrm{NaS}$ batteries answer the challenges of location dependence and material abundance issues; however, it presents its own special set of challenges. Sodium and sulfur are abundant but, in their molten liquid form, extremely dangerous. Pure sodium spontaneously burns on contact with air and moisture. In 2011, the Japan Mitsubishi Materials Corporation plant caught fired due to a $2000 \mathrm{~kW} \mathrm{NaS}$ battery [102]. The danger 
inherent with a $\mathrm{NaS}$ battery requires special consideration in the plant design, increasing cost. To compound that challenge, the harsh environment of a NaS battery is extremely corrosive. In addition, dendritic sodium growth can occur leading to damage in the ceramic separator and a battery short.

RFBs were tailor made for stationary, long-term, and high capacity energy storage making them ideal for grid energy storage. Unfortunately, RFBs are not without their own set of challenges. There are many different iterations of the RFB, which will be outlined in more detail in the following chapter. In some iterations, the redox active species themselves are too expensive to be cost competitive on a grid energy storage platform. On the sentiment of cost, the separator utilized in most aqueous RFBs is expensive. Much research has gone into driving down that cost but, it always comes with a sacrifice in efficiency. In other iterations, the primary challenge is dendritic growth of one or both active species as cycling continues. After some time, the dendrite can pierce the separator resulting in an short circuit of the battery where both electrodes are in contact. Methods have been developed to limit or reverse dendrite growth; however, none completely solve the problem while considering overall system cost and longevity.

The use of RFBs to solve the problem of grid energy storage is promising regarding their ability to separate energy and power, along with published results of high energy efficiency and a long lifetime; however, several hurdles remain in the push to control the market. According to Soloveichik, current research in RFBs should focus on the following challenges: finding a better redox couple, fundamental understanding of electrochemical processes, evaluation of environmental concerns, and further development of ion-exchange membranes, cell/stack design, and computational models [90]. 
The major disadvantage with a hybrid RFB is that now, power and energy are no longer decoupled. The amount of energy stored is dependent on the accessible plating volume which is dependent on the size of the electrochemical cells and stack. Some additional plating volume can be obtained by increasing the gap between electrode and ionexchange membrane; however, this comes with an efficiency cost due to ion transport and electrolyte conductivity that varies with the charge state.

A slurry electrode shows promise in again separating the power and energy of a hybrid RFB while providing additional reactant surface area. Unfortunately, slurry electrode must balance high viscosity with conductivity. The slurry particles must exist at a concentration that crosses the conductivity threshold. Unfortunately, this conductive slurry electrode tends to be highly viscous with increasing viscosity as the particles are plated. Viscosity creates two problems, first it causes more pump work reducing system efficiency and second, it brings about a new failure mode which is clogging of the electrochemical cell. Another challenge is that of plating distribution. For the slurry RFB to function properly, plating should occur on the slurry particles. However, plating can occur on the stationary current collector, resulting in a hybrid RFB instead of a slurry RFB.

A non-aqueous RFB can obtain a desirable energy efficiency and cell potential as observed in the examples above. However, the operating current density, and so the power density is generally much lower than their aqueous counterparts. Additionally, nonaqueous solvents tend to flammable, harmful to the environment, and expensive compared to water. Difficulties facing organic RFBs include limitations in cost, safety, environmental friendliness, electrode materials, and large-scale fabrication methods. Theoretically, organic molecules are cheap in terms of the elements that make them up but, considering 
the equipment and fabrication methods required to make them, they are considered expensive. This price should be driven down substantially with large-scale fabrication methods but, those methods are yet to be determined.

Membraneless RFBs remove one of the significant cost factors in a RFB, the ion exchange membrane. The price paid for reducing RFB cost is efficiency. As shown in the example above and in other literature, energy efficiency in membraneless RFBs tends to be extremely low. Additionally, they operate at very low current densities resulting in low power densities. These factors make membraneless RFBs impractical for grid energy storage currently.

For the widely commercialized all-vanadium RFB, the cost of the active species, $\mathrm{V}$, is the largest contributors to the capital cost of the system, see Figure $5[21 ; 103]$. The cost of the active redox species significantly effects the capital cost of a RFB system due to the volume of electrolyte necessary for grid energy storage. Currently, most research on the all-vanadium RFB is attempting to increase system performance by modifying the electrodes to increase overall energy efficiency and power density. These improvements will help to further reduce costs; but, only in small increments. Therefore, to continue the drive toward widespread commercialization of RFBs, another redox couple, that is cheaper than $\mathrm{V}$, should be studied and optimized to produce a comparable system performance. 


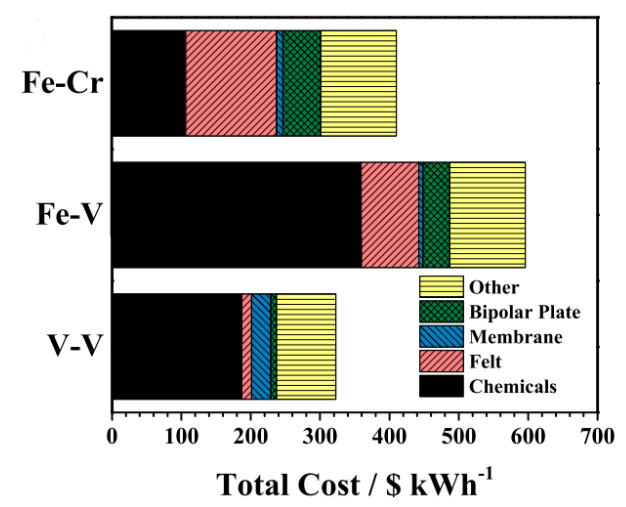

Figure 5. Component cost for a $1 \mathrm{MW}, 4 \mathrm{MWh}$ RFB of the listed chemistries [20].

The potentials at which the redox couples operate is one of the most important aspect of choosing active species. The potential difference of the two redox couples should be as high as possible to increase the power density of the RFB, which results in a smaller system size. The limit on the position of the redox couples' potentials is set by the potential at which solvent breakdown occurs. In aqueous solutions, this is referred to as water electrolysis. The potential range bounded by solvent breakdown is termed the solvent window. At a $\mathrm{pH}$ of zero, the gas evolution half reactions in water are as follows

$$
\begin{array}{cc}
\mathrm{O}_{2}+4 \mathrm{H}^{+}+4 e^{-} \rightleftharpoons 2 \mathrm{H}_{2} \mathrm{O} & 1.229 \mathrm{~V}(\mathrm{vs} . \mathrm{SHE})(3) \\
2 \mathrm{H}^{+}+2 e^{-} \rightleftharpoons \mathrm{H}_{2} & 0.00 \mathrm{~V}(\text { vs. SHE) (4) }
\end{array}
$$

In RFB research, gas evolution is typically considered an unwanted side reaction and represents a loss in energy efficiency. Capturing and utilization of evolved gases can be done; however, it adds another level of complexity to an RFB system that is typically avoided as it increases system cost in terms of capital cost and maintenance. The potential 
at which redox couples operate should, theoretically, be within the limits, shown above, set by gas evolution. However, it is not this unequivocal as there are other contributions to the potential at which gas evolution occurs. That is, the electrode can have a significant effect on the potential of gas evolution by introducing overpotentials to the gas evolution reaction. An overpotential is any extra potential, above the standard potential, required for a reaction to proceed. The traditional electrode chosen for RFBs is the graphite felt electrode which has a high overpotential to gas evolution in aqueous solutions. As a result, the potential at which a redox couple operates can extend well beyond its standard potential and cross over into the gas evolution territory. For example, in the all-vanadium cell, the standard potential of the $\mathrm{V}^{2+} / \mathrm{V}^{3+}$ redox couple is -0.255 (vs. SHE) which is below that of HER at low $\mathrm{pH}$. Because of this, the reaction at the anode of an all-vanadium RFB competes with HER.

Finding suitable redox chemistries for use in a RFB is complex. Redox couples must be low cost, have good energy density, high reversibility, good reaction kinetics, have reasonable potentials (which are inside the gas evolution potentials of aqueous solutions on the electrode of choice), and be resistant to and/or amenable to crossover. Energy density is dependent on the solubility of the active species, the number of electrons transferred per reaction, and the overall potential difference of the electrochemical cell. The redox species need to have high solubility. Solubility is not only limited by the solubility limit of the active species but, also by the viscosity of the solution. A highly viscous solution is undesirable as it will decrease ionic conductivity be increasing the diffusion coefficient and increase the cost of the pumping system beyond practical values. Multi-electron transfer reactions are preferred as they increase the effective energy density; however, they are often limited by reaction kinetics as multi-electron transfer reactions are 
more complex. Good reaction kinetics correlates to a higher charge and discharge current density. Also, high reversibility generates high efficiencies. Both characteristics, kinetics and reversibility, are dependent on the relationship between the active species and the electrode. High efficiency and good reaction kinetics contribute significantly to cost by reducing the size of the RFB system necessary to fit specific energy and power requirements.

Several challenges exist in the use of BDD electrodes for RFBs. Perhaps the most glaring is the ability to fabricate BDD films in a cost-effective way. Diamond is notorious for the price tag it carries. However, several factors point to a promise of low-cost BDD electrodes. First and foremost is the abundance of carbon. Carbon is readily available in many shapes and forms. The form commonly utilized as a carbon source for microwave plasma-assisted chemical vapor deposition (MPCVD) is methane. Methane is relatively abundant and even exists in the atmosphere. We can obtain it from many sources from dairy farms to the reduction of $\mathrm{CO}_{2}$. Additionally, the diamond used as an electrode can exist as a thin film, with a thickness of several nm, on a more easily fabricated substrate, such as graphite. Boron, on the other hand, is higher cost then methane, fortunately the amount of $\mathrm{B}$ required in BDD is very small compared to $\mathrm{C}$, less than $0.06 \% \mathrm{~B}$ atoms by at.\% to achieve metal-like conductivity.

The fabrication method used in this manuscript, MPCVD, requires a significant amount of energy and time to produce BDD films. However, other methods for fabricating BDD already exist. Hot filament chemical vapor deposition (HFCVD) can be used to make BDD films and can be engineered to grow thin films in a conveyer belt fashion. HFCVD does not typically generate the purity to produce gem quality, single crystal diamond but, 
the quality is acceptable for large scale fabrication acceptable electrode BDD thin films. In addition, the diamond industry is pushing the advancement of fabrication technology as each company fights for a market share of the ever-growing industry. From gem quality diamond to power electronics and even power tools, diamond is emerging in many facets of everyday life. Based on the maturity of the technology and the demand for its products, it is imagined the cost of thin film BDD fabrication will be continually drive down for years to come.

\subsection{Current Outlook}

Grid energy storage is currently a hot topic with no clear front runner to solving the demand in place by intermittent renewable energy sources. There is a wide variety of potential grid energy storage technologies, as demonstrated previously. Each of these technologies is being explored by multiple groups worldwide with some technologies, such as Li-ion and RFB, commanding the attention of hundreds of researchers.

In addition to energy storage of intermittent renewable energy sources, grid energy storage technologies are in demand for other reasons. Grid energy storage can increase the value of renewable energy by utilization as a load leveling device. Grid energy storage technologies can help augment power from renewable sources to maintain the proper specifications needed for entry into the power grid. Grid energy storage can assist the aging power infrastructure in the US and other countries by keeping energy storage on the load side of transmission constraint points, which makes the grid more secure, reliable, and responsive [38]. Grid energy storage also assists the aging infrastructure by reducing linecongestion and line-loss through moving electricity at off-peak times, which reduces 
generation requirements during peak times [38]. This effect reduces the power load of transmission and distribution lines during peak hours which can extend the life of existing infrastructure.

Grid energy storage may also benefit the advancement of electric transportation. Grid energy storage technologies are generally non-polluting and, in some cases, nondestructive to the environment. This allows for more relaxed regulations on the placement of grid energy storage sites. As a result, they can be placed closer to cities and residential areas. This would greatly facilitate the movement of transportation services to electricity power by extending the spread of "plug-in" points throughout the country. Grid energy storage may also be significant in emergency preparedness by increasing the vitality of our power grid. In addition, grid energy storage can enhance the resilience and robustness of our power grid when considering weather outages and other potential disruptions. For the reasons outlined here, grid energy storage is poised to grow dramatically in the coming years.

Currently, pumped hydro and CAES systems have the lowest cost in terms of $\$ \mathrm{kWh}^{-1}$, while pumped hydro is a mature technology with a high energy efficiency [63]. However, the maturity of the technology and the long timeline for development means the cost of pumped hydro is unlikely to be significantly reduced in the short term. Grid scale demonstration of CAES is still necessary to prove its viability. It also requires a significant timeline for development. Li-ion offers the best grid energy storage option when considering together cost, cycle life, and maturity [63]. The maturity of Li-ion is attractive in that, it is well known what to expect from these systems; however, realizing cost reductions may be difficult. NaS batteries are expected to significantly reduce capital costs 
associated with grid energy storage. Unfortunately, this technology is still in its infancy and, considering the associated hazards, the future is unknown.

RFBs are often considered the up and coming front runner to Li-ion technology. It is well known that, due to its efficiency, lifetime, and maturity, pumped hydro will be around for years to come; however, based on its location dependence and environmental impact, widespread use of this technology in terms of grid scale energy storage for intermittent renewable energy is unlikely. In terms of maturity, RFB technology is in between $\mathrm{Li}$-ion and $\mathrm{NaS}$ batteries. It is mature enough to have already been implemented as a grid scale energy device in multiple locations throughout the world. Yet, there is still room for significant improvement in terms of technological advancements as well as optimization of the electrochemical cell itself and the battery management system. Rapid improvements in cost, performance, life, and technological and manufacturing readiness are expected in the coming years [63].

The outlook for RFBs is extremely promising. Pumped hydro storage, which currently holds the lion's share of energy storage applications, cannot support grid energy storage for the expanding renewable energy sector due to its location dependence. As a result, renewable energy producers are increasingly turning to emerging technologies as an alternative. These alternatives have primarily been Li-ion batteries and RFBs. Li-ion batteries are a good choice for the reasons outlined previously in Chapter 2; however, they do not currently result in a cost competitive overall grid energy system when compared to non-renewable sources. In addition, the maturity of the technology leaves little room for significant improvement in terms of functionality and more importantly cost. As electric vehicles gain popularity and more Li-ion batteries are fabricated, cost will become an ever- 
increasing concern due to the relative rarity of Li on Earth. So, renewable energy producers are poised to embrace RFBs in terms of grid energy storage.

RFB technology has matured to the level of several grid scale implementations throughout the world. Even so the technology has a vast amount of room to grow due to technological advancements as well as optimization. Currently, RFB technology leans heavily on materials and processes that were developed for other technologies such as the Nafion membrane from hydrogen/oxygen fuel cells. These membranes are expensive and focus on the transfer of hydrogen ions while rejecting all other species transport. However, a RFB utilizes liquid phase electrolytes instead of gases and these electrolytes can be made from a wide variety of active species, solvents, and supporting electrolytes. As a result, Nafion may be far from ideal for a RFB system. There are an overwhelming number of avenues to explore when considering RFB research. In addition, optimization of RFBs has only scratched the surface. Advancements are continually being made in the proper charge/discharge regimes implemented into real world applications. Flow cell shapes and designs are another source of optimization, along with the method of electrode incorporation.

Utilizing a low-cost active species would significantly drive the price of the RFB system down. Finding suitable redox chemistries for use in a RFB is complex. Redox couples must be low cost, have good energy density, high reversibility, good reaction kinetics, have reasonable potentials (which are inside the gas evolution potentials of aqueous solutions on the electrode of choice) and be resistant to and/or amenable to crossover. Energy density is dependent on the solubility of the active species, the number 
of electrons transferred per reaction, and the overall potential difference of the electrochemical cell.

The redox species need to have high solubility. Solubility is not only limited by the solubility limit of the active species but, also by the viscosity of the solution. A highly viscous solution is undesirable as it will decrease ionic conductivity be increasing the diffusion coefficient and increase the cost of the pumping system beyond practical values.

Multi-electron transfer reactions are preferred as they increase the effective energy density; however, they are often limited by reaction kinetics as multi-electron transfer reactions are more complex. Good reaction kinetics correlates to a higher charge and discharge current density. Also, high reversibility generates high efficiencies. Both characteristics, kinetics and reversibility, are dependent on the relationship between the active species and the electrode. High efficiency and good reaction kinetics contribute significantly to cost by reducing the size of the RFB system necessary to fit specific energy and power requirements. 


\section{CHAPTER 3}

\section{EXPERIMENTAL}

\subsection{Electrochemical testing setups}

Several testing setups will be utilized including a full RFB system, epoxied electrodes, and multi-port boiling flask. Epoxied electrodes will be used as a straightforward method for electrochemical testing of BDD thin films. They allow for standard electrochemical tests such as CV and EIS; however, this method is limited in its ability to represent scale up. The multi-port boiling flask testing setup was developed to avoid using epoxy, which is impossible to remove without damaging or at least modifying the electrode surface. The multi-port boiling flask testing setup has the same abilities as the epoxied electrodes method but, the electrodes can be removed after testing and recharacterized to determine changes brought on by electrochemical methods. The full RFB system will allow for charge/discharge testing of an actual RFB. In this research, the RFB will be limited to a single cell. Scale up to multiple cells can be realized with similar results; however, additional considerations outside of the scope of this research must be accounted for. These included leaching currents across cells, ohmic resistances, and sealing of multiple cells. 


\subsubsection{Epoxied electrodes}

To begin initial testing on BDD electrodes, an alternative to a typical RFB electrochemical cell must be utilized. BDD electrode samples fabricated for this research are around $1 \mathrm{~cm}^{2}$ in size. Modifying them for use in a full RFB and assembling the RFB each time a new test is performed is not practical. So, the BDD samples are modified by affixing a conductive wire to them and attaching them to a glass tube with epoxy, an example electrode is shown in Figure 6. For BDD on silicon substrates, the back of the electrode, where bare silicon resides, is scratched with a diamond scribe to remove the oxide layer. A gallium and indium eutectic is placed on the scratched surface forming ohmic contact with the conductive silicon substrate. Then a coiled wire is placed in the gallium and indium eutectic and silver paste is painted on top. The silver paste serves as a conductive and protective layer to cover and hold the coiled wire in the gallium and indium eutectic so that a stronger epoxy can be layered on top. A chemically and electrically resistant epoxy is used to cover the BDD sample while attaching it to a glass tube that also contains the wire. The wire runs through and out of the glass tube and is used as the electrical connection to the testing equipment. Additionally, the epoxy forms a window on the BDD surface which defines the electrochemical surface area available for reaction. The result is a stick like electrode that can be inserted into an electrolyte solution for testing and easily removed. Metal substrates were prepared in almost the same way, less the oxide layer scratching and eutectic. 


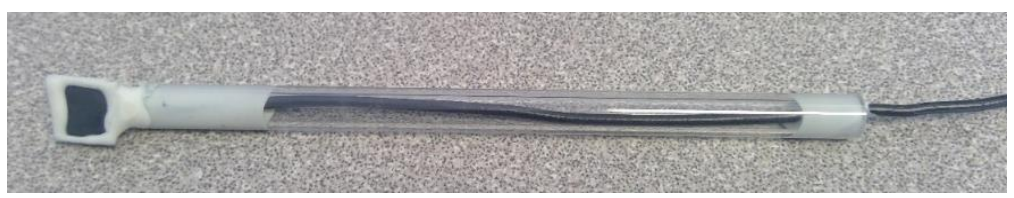

Figure 6. Example of an epoxied electrode. The active surface area on the left side is defined by the applied epoxy.

\subsubsection{Multi-port boiling flask}

The epoxied electrode works well for electrochemical testing; however, its limitation is in the permanence of the epoxy. It is impossible to remove the epoxy without damaging the sample itself. As a result, non-electrochemical characterization of the BDD sample is difficult or impractical to perform. To solve this issue, another testing apparatus was developed which utilizes a boiling flask with multiple ports, shown in Figure 7. Two of these ports are used for filling the electrolyte, depending on the orientation of the flask. Three ports are used for reference and counter electrodes as well as an optional gas bubble or blanket to prevent reaction of the electrolyte with the outside air. The last port is an Oring joint that is placed on top of the BDD sample. A Viton O-ring and a horseshoe clamp will allow for sealing of the BDD sample against the boiling flask. The O-ring joint defines the active area of the BDD electrode surface which is exposed to the electrolyte. This testing apparatus does not permanently affix and/or deform the BDD sample so, it can easily be taken out and characterized before and after each test. 


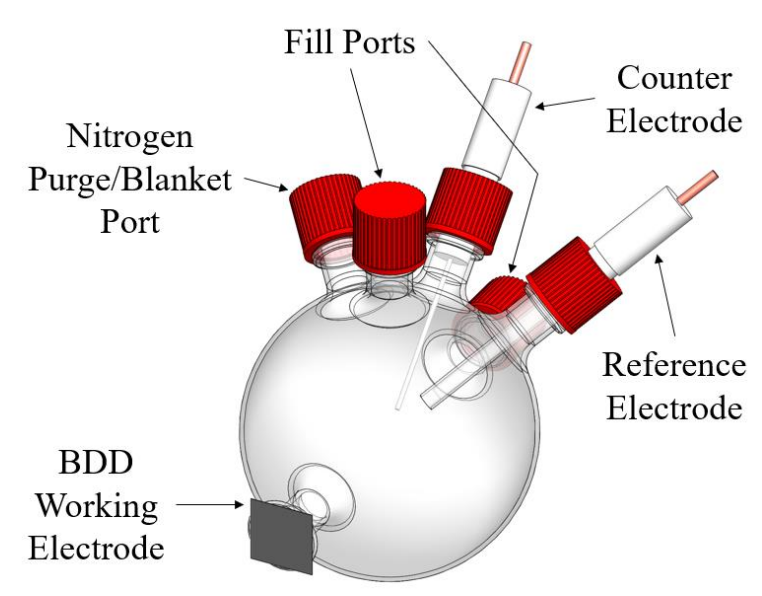

Figure 7. Multi-port boiling flask with 3 ports for reference electrode, counter electrode, and gas flow, 2 ports for filling of electrolyte solution, and one port for the working electrode.

\subsubsection{Full cell}

The full RFB system includes everything that is used in a real RFB system ending at the external source/sink. That is, the full RFB system includes end plates, conduction plates, flow plates, gaskets, electrodes, an ion exchange membrane, storage reservoirs, tubing, and all necessary connections. The full RFB, shown in Figure 8, used for testing in this study was completely designed and fabricated in house. The primary function of the end plates is to compress the cell assembly to ensure good electrical contact between components and to seal the liquid electrolyte so that it does not leak. In this design, the end plates are made of thick PVC. The conduction plate, made of copper here, is used to connect the cell to an external source/sink for charge/discharge. The flow plates are made of graphite, as they contact the electrolyte fluid; they must be inert and conductive. The flow plates route the flow of electrolyte to the electrode and move electrons in/out of the electrolyte from the source/sink. In the design shown in Figure 8, the flow pattern is a 
simple open pocket. Inside the pocket, the electrode, such as graphite felt, is placed. Compression of the electrode leads to good electrical contact with the flow plate. Other common flow plate designs include serpentine and interdigitated channels.

The gaskets in this RFB are Viton, which is compressible and chemically resistant. The storage reservoirs hold the electrolyte after charge/discharge and are simple polypropylene bottles. They come in various volumes and are chemically resistant. Only one storage reservoir is used for the anolyte and one for the catholyte. This means, during charge and discharge, the full volume of the electrolyte is cycled through several times. This is useful as all the ions are not reduced or oxidized during a single pass unless the flow rates are slow enough; however, slow flow rates result in a lower efficiency due to low diffusion. Higher flow rates promote turbulence, which results in more active species reaching reaction sites. A peristaltic pump was employed as the pump components do not contact the fluid, only the tubing.

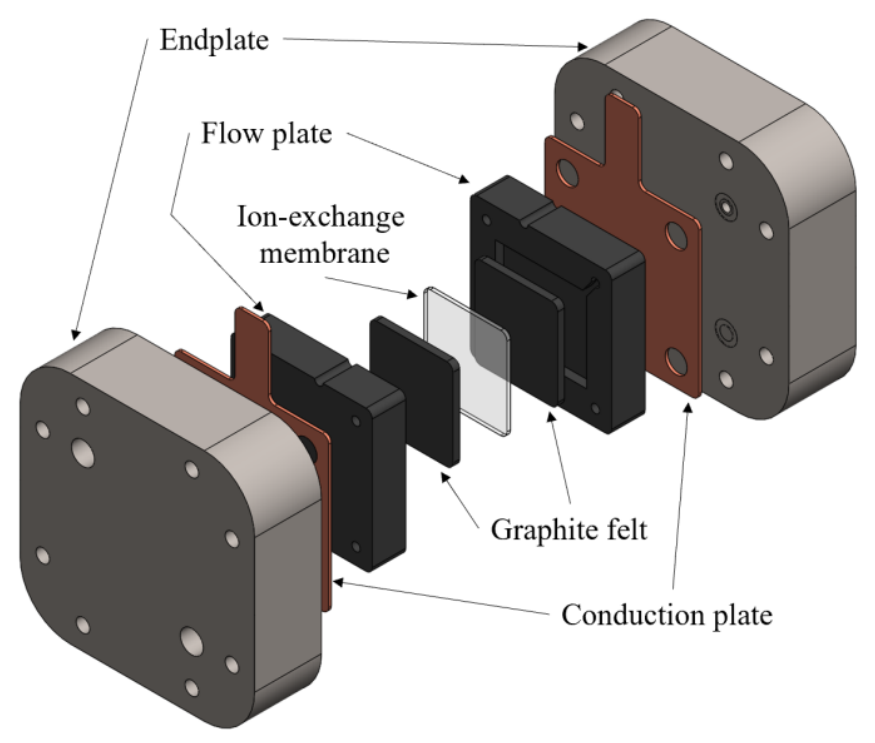

Figure 8. Full RFB designed and fabricated at the University of Louisville. 


\subsection{Materials}

BDD electrodes were prepared by microwave plasma-enhanced chemical vapor deposition (MPCVD) using $\mathrm{H}_{2}, \mathrm{CH}_{4}$, and $\mathrm{B}_{2} \mathrm{H}_{6}$ as the plasma gas, $\mathrm{C}$ source, and $\mathrm{B}$ doping source, respectively. The substrate was p-type, $\langle 100\rangle$, highly conductive $\mathrm{Si}$ with a resistivity of $0.001-0.005 \Omega \mathrm{cm}$. The BDD sample quality was characterized by Raman spectroscopy to verify the Raman shift peak is at the correct wavenumber $\left(1332 \mathrm{~cm}^{-1}\right)$ and scanning electron microscopy (SEM) to verify the diamond crystal morphology. A new BDD sample, each grown using the same pressure, power, flow rates, and duration, was used for each redox couple to prevent possible cross contamination issues. Analysis of randomly chosen samples from different growth batches verified there was no observable change in properties.

The Ce-based electrolytes were prepared by dissolving $\mathrm{Ce}\left(\mathrm{NO}_{3}\right)_{3} \cdot \mathrm{xH}_{2} \mathrm{O}$ (Alfa Aesar, Haverhill, MA, USA, 99.998 \%) in $\mathrm{HNO}_{3}$ (Sigma-Aldrich, St. Louis, MO, USA, ACS reagent) or $\mathrm{H}_{2} \mathrm{SO}_{4}$ (VWR, Radnor, PA, USA, 95-98 \%), and $\mathrm{Ce}_{2}\left(\mathrm{CO}_{3}\right)_{3}$ (Acros Organics, Pittsburgh, PA, USA, $99.9 \%$ ) and $\mathrm{Ce}\left(\mathrm{SO}_{4}\right)_{2}$ (Acros Organics, Pittsburgh, PA, USA, $99 \%$ ) in $\mathrm{H}_{2} \mathrm{SO}_{4}$. The Mn-based electrolytes were prepared by dissolving $\mathrm{MnSO}_{4} \cdot \mathrm{H}_{2} \mathrm{O}$ (Sigma-Aldrich, St. Louis, MO, USA, $\geq 98 \%$ ) in $\mathrm{HNO}_{3}$ or $\mathrm{H}_{2} \mathrm{SO}_{4}$, $\mathrm{Mn}\left(\mathrm{NO}_{3}\right)_{2} \cdot 4 \mathrm{H}_{2} \mathrm{O}$ (Sigma-Aldrich, St. Louis, $\mathrm{MO}$, USA, $\geq 97 \%$ ) in $\mathrm{HNO}_{3}$, and $\mathrm{Mn}\left(\mathrm{ClO}_{4}\right)_{2} \cdot \mathrm{xH}_{2} \mathrm{O}$ (Sigma-Aldrich, St. Louis, MO, USA, $99 \%$ ) in $\mathrm{HClO}_{4}($ Sigma-Aldrich, St. Louis, MO, USA, ACS reagent). The Fe-based electrolyte was prepared by dissolving $\mathrm{Fe}_{2}\left(\mathrm{SO}_{4}\right)_{3} \cdot \mathrm{xH}_{2} \mathrm{O}$ (VWR, Radnor, PA, USA, reagent) in $1 \mathrm{M} \mathrm{H}_{2} \mathrm{SO}_{4}$. The I-based electrolyte was prepared by dissolving KI (Alfa Aesar, Haverhill, MA, USA, $99 \%$ ) in $1 \mathrm{M} \mathrm{KCl}$ (Sigma-Aldrich, St. Louis, MO, USA, $99.99 \%$ ). The Fe(CN)6-based electrolyte was 
prepared by dissolving $\mathrm{K}_{3}\left[\mathrm{Fe}(\mathrm{CN})_{6}\right]$ (Sigma-Aldrich, St. Louis, MO, USA, $99 \%$ ) in $1 \mathrm{M}$ KCl (Sigma-Aldrich, St. Louis, MO, USA, $99.99 \%$ ). The Cu-based electrolyte was prepared by dissolving $\mathrm{CuSO}_{4} \cdot 5 \mathrm{H}_{2} \mathrm{O}$ (VWR, Radnor, PA, USA, ACS reagent) in $3 \mathrm{M}$ $\mathrm{H}_{2} \mathrm{SO}_{4}$. All electrolytes were prepared with DI water $(>18.2 \mathrm{M} \Omega \mathrm{cm})$. All electrolyte solutions were bubbled with $\mathrm{N}_{2}$ for 30 min to remove dissolved $\mathrm{O}_{2}$ prior to measurements.

\subsection{Instruments}

BDD samples were grown using a MPCVD reactor (SEKI Technotron-ASTeX AX5200S-ECR or SEKI Technotron-ASTeX AX5010). Images of the samples were first obtained using an optical microscope (Zeiss Axio Imager.A2m). The samples were then imaged with a scanning electron microscope (SEM) to better determine the morphology, crystal size, and thickness/growth rate (FEI Nova600 FEG-SEM or TESCAN Vega3 SEM). To qualitatively determine the purity and B doping concentration of the BDD samples, they were characterized with Raman (Reinshaw inVia confocal Raman/PL) and FTIR (PerkinElmer Spectrum BX FTIR). Electrochemical testing was done using two different potentiostats (Biologic SP200, PGSTAT 128N) both with electrochemical impedance spectroscopy (EIS) capabilities. After charge/discharge testing of the allvanadium RFB, electrolyte samples were examined using UV-vis (Perkin Elmer Lambda 950). After electrochemical testing of the BDD samples using the $\mathrm{Mn}^{2+} / \mathrm{Mn}^{3+}$, the samples were characterized using energy-dispersive X-ray spectroscopy (EDS) (TESCAN Vega3 SEM with EDAX) to determine the amount and/or presence of $\mathrm{MnO}_{2}$ on the BDD surface. 


\subsection{Fabrication and demonstration of a RFB}

To understand the challenges involved in the operation and optimization of a RFB, it is necessary to build and test a RFB. Therefore, the first objective is to demonstrate the ability to fabricate and operate a RFB. To this end, a RFB was designed and fabricated inhouse. The full RFB testing setup is seen in Figure 9. Achieving published values for the all-vanadium RFB is essential to beginning research on RFBs in general. This accomplishment will give a better understanding of RFBs while building up confidence to continue more detailed analysis and modification of RFB systems.

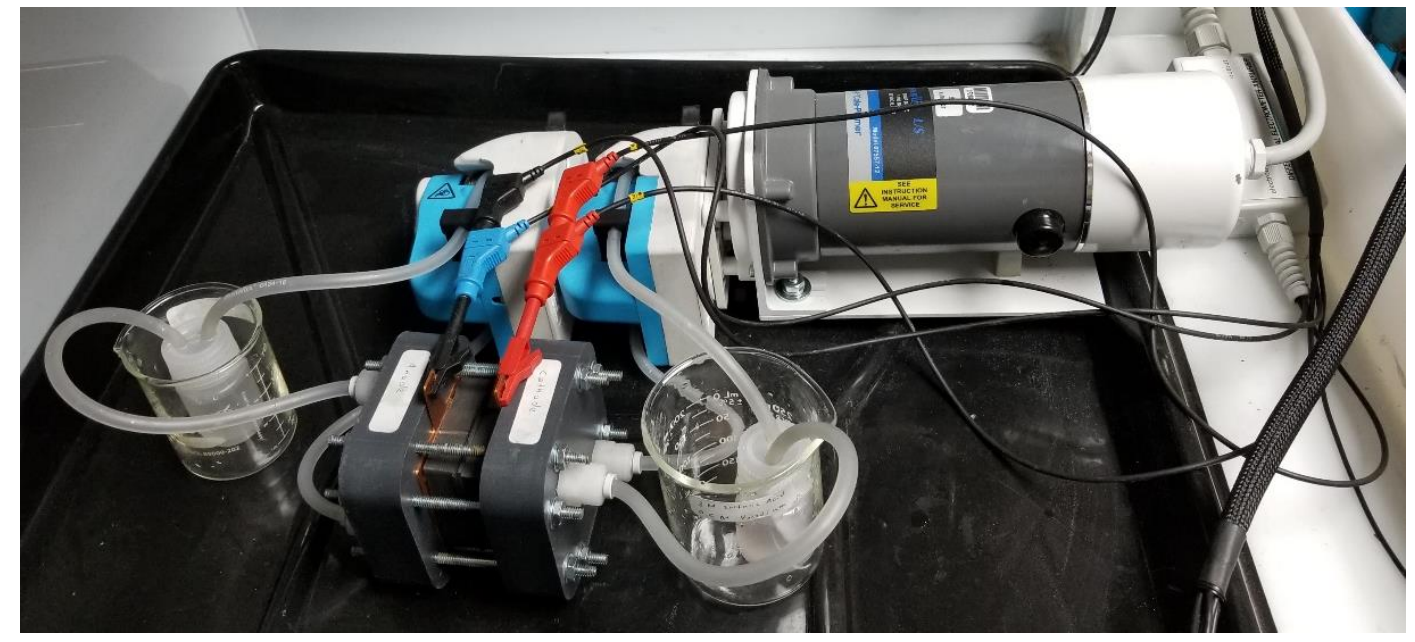

Figure 9. Full RFB testing setup complete with two storage reservoirs and a peristaltic pump.

\subsubsection{All-vanadium}

The all-vanadium chemistry was chosen to explore the full RFB test setup as it is the most published redox chemistry in the RFB field and so, it provides the most data to be utilized for exploration, comparison, and troubleshooting. The overall theoretical potential 
of this system is $1.246 \mathrm{~V}$. This work will lay the groundwork for the achievement of other objectives and provide a basis for comparison using alternate chemistries and electrodes.

The starting material to make the electrolyte for the all-vanadium RFB is typically vanadyl sulfate $\left(\mathrm{VOSO}_{4}\right.$, which provides the $\mathrm{V}^{4+}$ species) hydrate. This is used as it comes in a powder form that readily dissolves in water and does not release any extra chemical species then are already present in the $\mathrm{H}_{2} \mathrm{SO}_{4}$ solution. It is possible to begin with $\mathrm{V}^{5+}$ in the form of $\mathrm{V}_{2} \mathrm{O}_{5}$; however, this requires an additional step using a strong reducing agent as $\mathrm{V}_{2} \mathrm{O}_{5}$ is not readily soluble in water. Reducing $\mathrm{V}_{2} \mathrm{O}_{5}$ may be more economical, as $\mathrm{V}_{2} \mathrm{O}_{5}$ is cheap compared to $\mathrm{VOSO}_{4}$ but, it is not ideal for testing as it requires more chemical species, the reducing agent, to be introduced into the system.

The all-vanadium RFB system works by cycling two vanadium redox couples: $\mathrm{V}^{2+} / \mathrm{V}^{3+}$ in the anolyte and $\mathrm{V}^{4+} / \mathrm{V}^{5+}$ in the catholyte. To obtain $\mathrm{V}^{3+}$ from $\mathrm{V}^{4+}$, electrochemical reduction at the anode, via constant current or constant voltage charging, must be performed. Figure 10 shows electrochemical reduction of $\mathrm{V}^{4+}$ to $\mathrm{V}^{3+}$ and then $\mathrm{V}^{3+}$ to $\mathrm{V}^{2+}$ via constant current charging. The transition point where all $\mathrm{V}^{4+}$ has converted to $\mathrm{V}^{3+}$ and, $\mathrm{V}^{2+}$ begins to be produced, appears as a noticeable bump in charge curve, resulting in an increased potential. At the cathode, during the charging process, $\mathrm{V}^{4+}$ is converting to $\mathrm{V}^{5+}$. Because of that, there must be twice the volume of $\mathrm{V}^{4+}$ in the cathode to start. After this process is performed, the system now contains charged species in the anode, $\mathrm{V}^{2+}$, and cathode, $\mathrm{V}^{5+}$. To bypass this initial charging step, vanadium chloride may be purchased with vanadium in the $\mathrm{V}^{2+}$ or $\mathrm{V}^{3+}$ oxidation state. Vanadium chloride is readily soluble in water; however, it releases chlorines into the solution which may not be ideal. For the 
purposes of this study, all starting solutions were made from vanadyl sulfate and sulfuric acid for use in the full RFB.

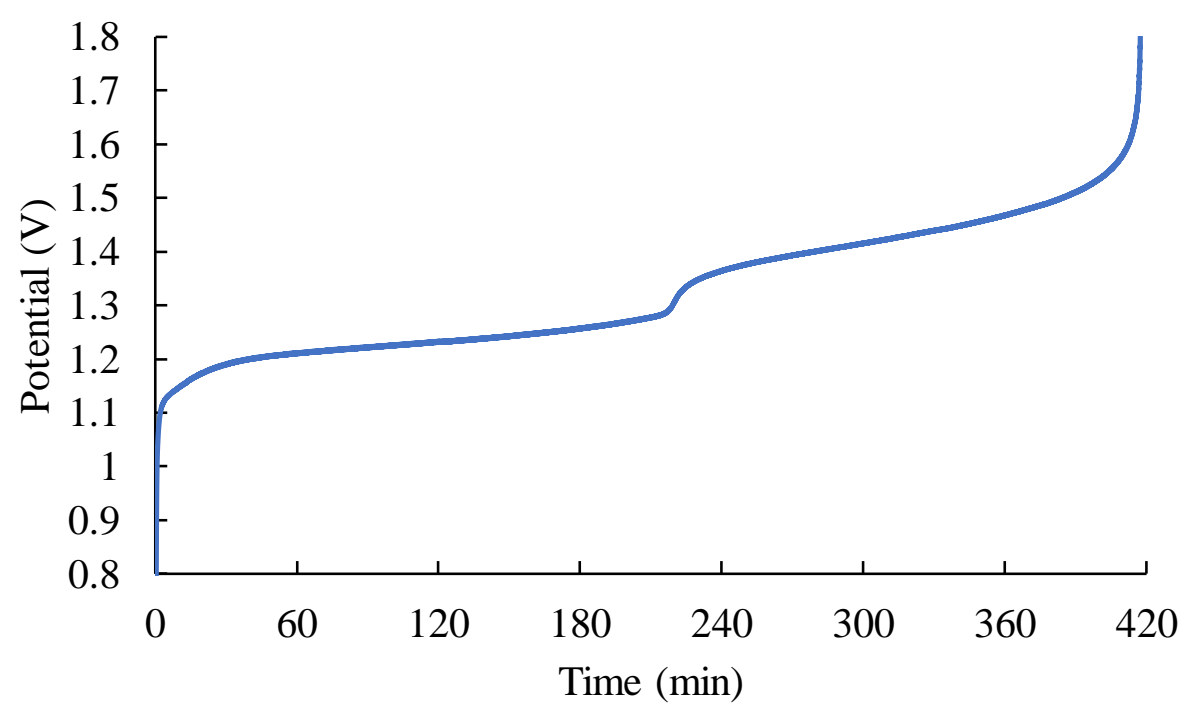

Figure 10. Initial charge curve to convert V(IV) to V(II).

The electrodes used for this testing were AvCarb G100 graphite felt with an area of $10 \mathrm{~cm}^{2}$, and the ion-exchange membrane was Nafion NRE 212. A common metric used to compare energy storage systems is the energy efficiency. Energy efficiency for a RFB is calculated using

$$
\mathrm{EE}=\mathrm{CE} * \mathrm{VE}
$$

where CE is the coulombic efficiency and VE is the voltage efficiency. When using the same charge and discharge current, the coulombic efficiency is calculated using 


$$
\mathrm{CE}=\frac{\mathrm{t}_{\mathrm{d}}}{\mathrm{t}_{\mathrm{c}}}
$$

where $t_{d}$ is the total discharge duration and $t_{c}$ is the total charge duration. Voltage efficiency is calculated using

$$
\mathrm{VE}=\frac{\mathrm{V}_{\mathrm{avg}, \mathrm{d}}}{\mathrm{V}_{\mathrm{avg}, \mathrm{c}}}
$$

where $V_{a v g, d}$ is the average discharge potential and $V_{a v g, c}$ is the average discharge potential. This flow battery exhibited great performance, achieving energy efficiencies as high as $86 \%$ at $20 \mathrm{~mA} \mathrm{~cm}^{-2}$. That is equal to, or better than, results from recent published literature, without optimization. Figure 11 shows the coulombic, voltage, and energy efficiency for the in-house fabricated RFB which underwent 49 cycles. The coulombic efficiency stayed near $94 \%$ for the duration of the cycling. The slight increase in the first few cycles is due to conditioning of the ion-exchange membrane. The voltage efficiency continually declined, losing about $1 \%$ over the duration of 49 cycles. This is a result of decreased ionic conductivity in the ion-exchange membrane, resulting from mass transport via migration of active species at high and low SOC. Active species transport through the ion-exchange membrane blocks transport pathways of $\mathrm{H}^{+}$ions, decreasing the ionic conductivity with each cycle. 


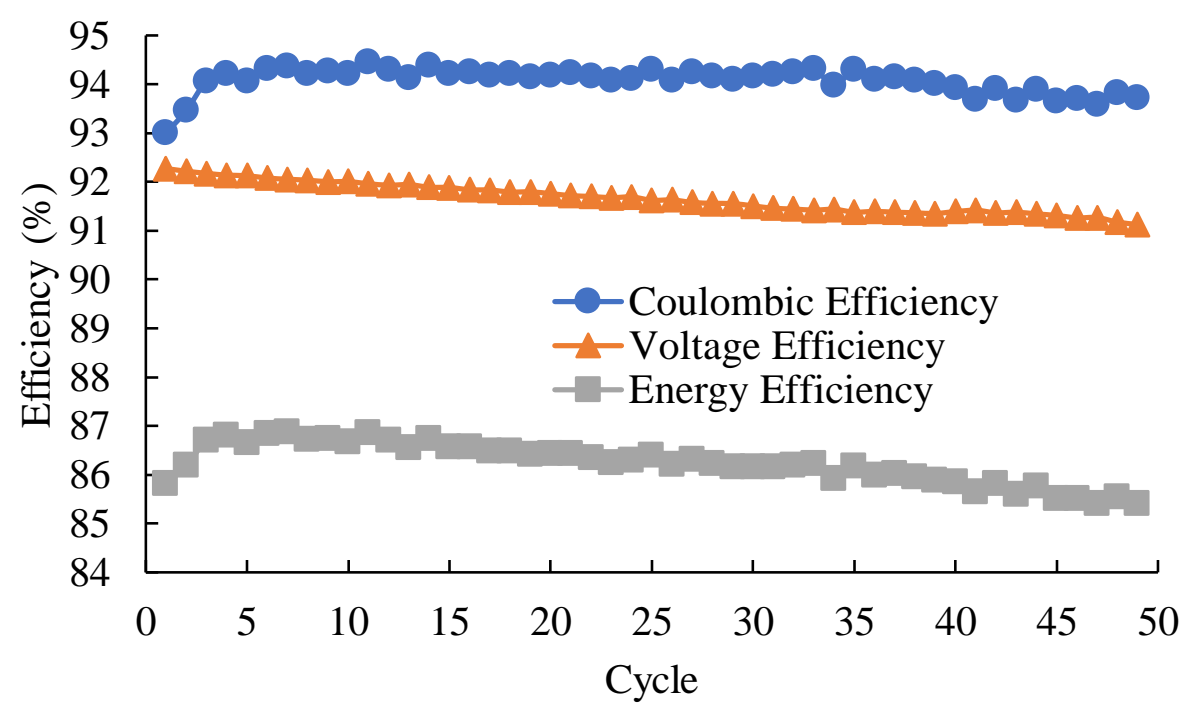

Figure 11. Coulombic, voltage, and energy efficiency of the in-house fabricated flow battery which underwent 49 charge/discharge cycles.

Capacity fade over the duration of 49 cycles for the in-house fabricated RFB is shown in Figure 12. Capacity fade is due to crossover of vanadium species from anolyte to catholyte, or vice versa. Crossover occurs in both directions in a RFB; however, the net flow is from anolyte to catholyte in the all-vanadium RFB due to the smaller particle size of the $\mathrm{V}^{2+}$ and $\mathrm{V}^{3+}$ ions. When an active species crosses the membrane, it will react with particles on the other side to equilibrate. Particles from the anolyte will want to oxidize and give up electrons when they move to the catholyte. This results in either the reduction of catholyte active species or the neutralization of the $\mathrm{H}^{+}$ions back into water molecules. The electrolyte volume for this RFB was $25 \mathrm{~mL}$. The initial capacity is small, and the capacity fade appears exaggerated. 


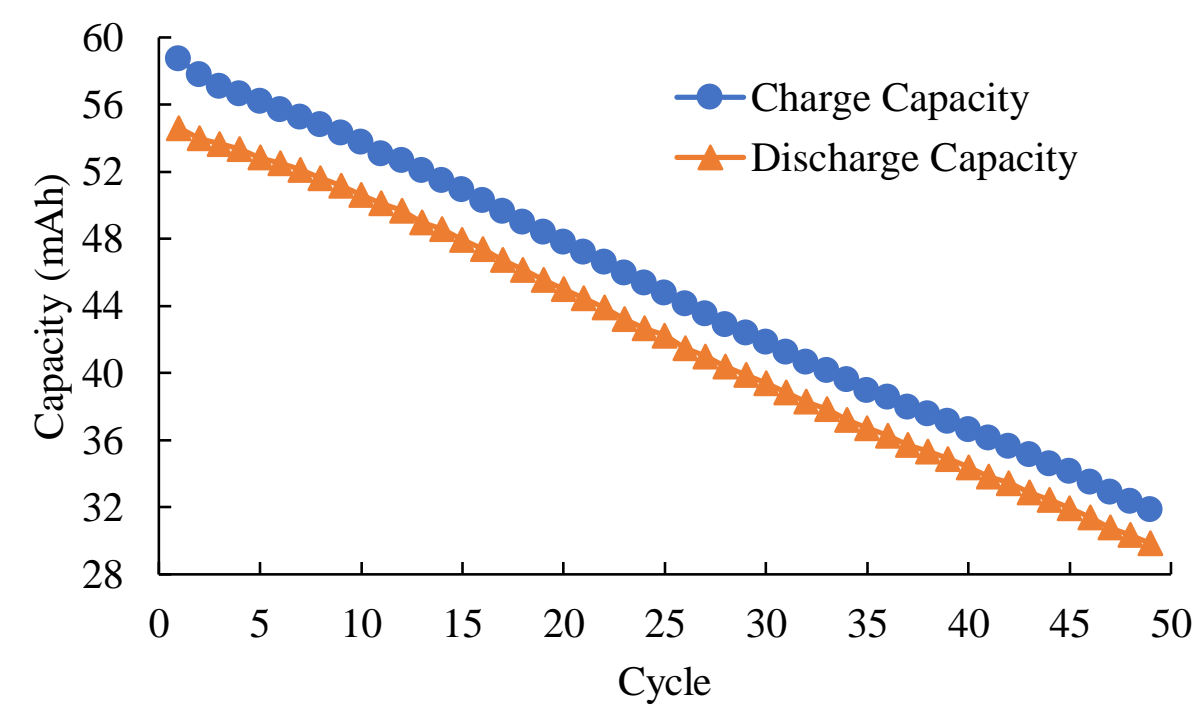

Figure 12. Charge and discharge capacity over 49 cycles using the in-house fabricated all-vanadium RFB.

The following images demonstrate the ability of UV-vis to determine the oxidation state of vanadium present in a sample. With this data the concentration of each species can be determined and, by extension, the state of charge of the electrolyte. Figure 13 shows UV-vis of a discharged anolyte. The anolyte, in its fully discharged state, should contain only $\mathrm{V}^{3+}$ ions. The UV-vis result is compared to a result derived from literature. The UVvis spectra compare well with literature and confirms the discharged state of the anolyte contains primarily the $\mathrm{V}^{3+}$ ion. 


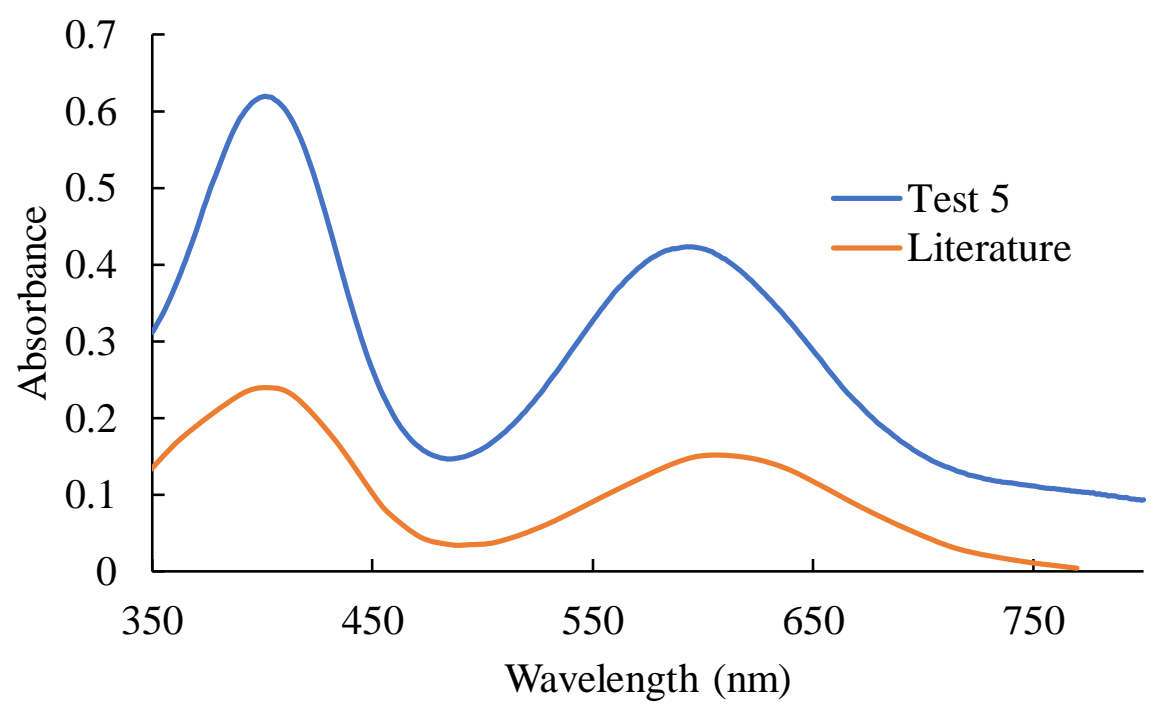

Figure 13. UV-vis of the discharged anode electrolyte. expected to be $\mathrm{V}^{3+}$, compared to literature [12].

The next image demonstrates the use of cyclic voltammetry (CV) in electrolyte analysis. Figure 14 shows five cycles of CV simultaneously, with a scan rate of $5 \mathrm{mV} / \mathrm{s}$, of the vanadium catholyte using graphite felt as the electrode. Any difference in the cycles are nearly imperceptible, indicating good stability while cycling. In this setup the counter electrode is a graphite rod and the reference electrode is $\mathrm{Ag} / \mathrm{AgCl}$. This test was performed with the electrodes suspended in a beaker filled with the electrolyte solution. Each of the five cycles overlaps the others very closely, this is indicative of good cyclability. The average potential of the two peaks gives the potential for the redox couple. For Figure 14, this value is $0.890 \mathrm{~V}$ vs. $\mathrm{Ag} / \mathrm{AgCl}(1.089 \mathrm{~V}$ vs. SHE). Using the potential for the cathode and anode redox couples, the expected potential for the full RFB is determined from CV analysis of the anolyte and catholyte. The peak separation indicates the reversibility of the electrode. The peak separation for the CV in Figure 14 was found to be $0.85 \mathrm{~V}$. The peak 
ratio of a CV curve can qualitatively indicate the coulombic efficiency of the system. Peak ratio is difficult to determine as it depends on the scan rate and the potential at which the curve changes direction. The peak ratio of the $\mathrm{CV}$ was calculated using

$$
\frac{i_{\mathrm{pa}}}{i_{\mathrm{pc}}}=\frac{\left(i_{\mathrm{pa}}\right)_{0}}{i_{\mathrm{pc}}}+\frac{0.485\left(i_{\mathrm{sp}}\right)_{0}}{i_{\mathrm{pc}}}+0.086
$$

where $i_{p a}$ is the anodic current peak and $i_{p c}$ is the cathodic current peak, and $i_{\text {sp }}$ is current at which the CV scan direction changes [9]. The peak ratio for the CV in Figure 14 was found to be 1.97. A peak ratio of unity is desired and indicates a high columbic efficiency can be obtained. The poor performance of the graphite felt in this $\mathrm{CV}$ analysis was a result of poor wetting of the graphite felt (due to a no flow condition). This has since been corrected by applying a vacuum to the graphite felt while it is in the electrolyte solution, which pulls out any air trapped in the porous felt. After this was done, the peak separation and peak ratio significantly reduced. 


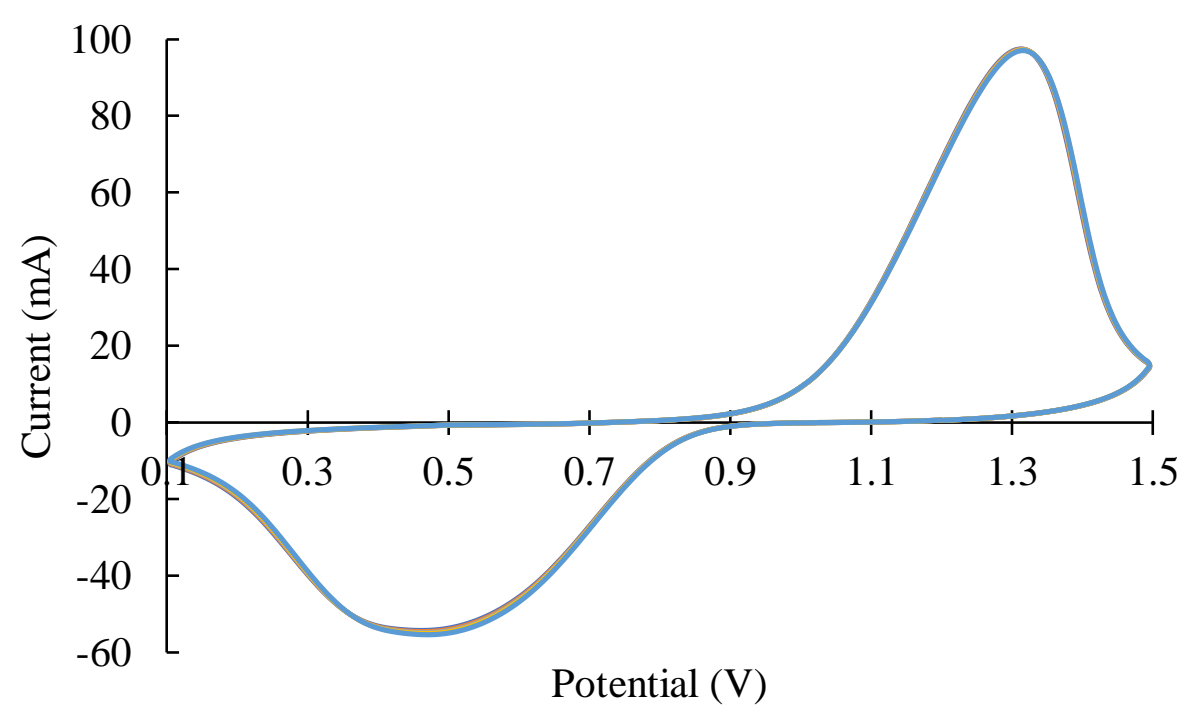

Figure 14. Five cycle $\mathrm{CV}$ of an unmodified graphite felt at a scan rate of $5 \mathrm{mV} \mathrm{s}^{-1}$ with $\mathrm{Ag} / \mathrm{AgCl}$ as the reference electrode.

\subsubsection{Vanadium/manganese}

The $\mathrm{V}^{2+} / \mathrm{V}^{3+}$ and $\mathrm{Mn}^{2+} / \mathrm{Mn}^{3+}$ redox couples will be used in the full RFB test setup to explore manganese as a cathode redox chemistry. Vanadium will be used as the anode redox chemistry because it is a well-known chemistry. Manganese is a favorable redox couple due to its low cost and high potential. The overall theoretical potential of this system is $1.797 \mathrm{~V}$. In preliminary findings it was found that manganese is a promising redox couple on a BDD electrode, warranting its exploration in a full RFB with a graphite electrode. Using the well know vanadium anolyte redox couple allows for a better understanding of the challenges present employing the $\mathrm{Mn}^{2} / \mathrm{Mn}^{3+}$ redox couple as the catholyte. This objective will aid in the understanding and use of manganese as a redox couple for RFBs.

The anolyte of the vanadium/manganese RFB was made up of $0.5 \mathrm{M} \mathrm{VOSO}_{4}$ in $3 \mathrm{M} \mathrm{H}_{2} \mathrm{SO}_{4}$. The manganese catholyte was $0.5 \mathrm{M} \mathrm{MnSO}_{4}$ in $3 \mathrm{M} \mathrm{H}_{2} \mathrm{SO}_{4}$. Charge and 
discharge cycles were performed with a current density of $20 \mathrm{~mA} \mathrm{~cm}^{-2}$. The electrodes and ion-exchange membrane were the same as previously used, graphite felt and Nafion, respectively. Figure 15 shows charge and discharge curves for 4 cycles. It is seen from the cycle data that the capacity reduces significantly with each cycle as the charge and discharge durations get lower. The average charge capacity over the 4 cycles is $163.7 \mathrm{mAh}$ while the average discharge capacity is $83.1 \mathrm{mAh}$. This is attributed to active species crossover and precipitation of $\mathrm{MnO}_{2}$ in the catholyte which decreases the concentration of active species.

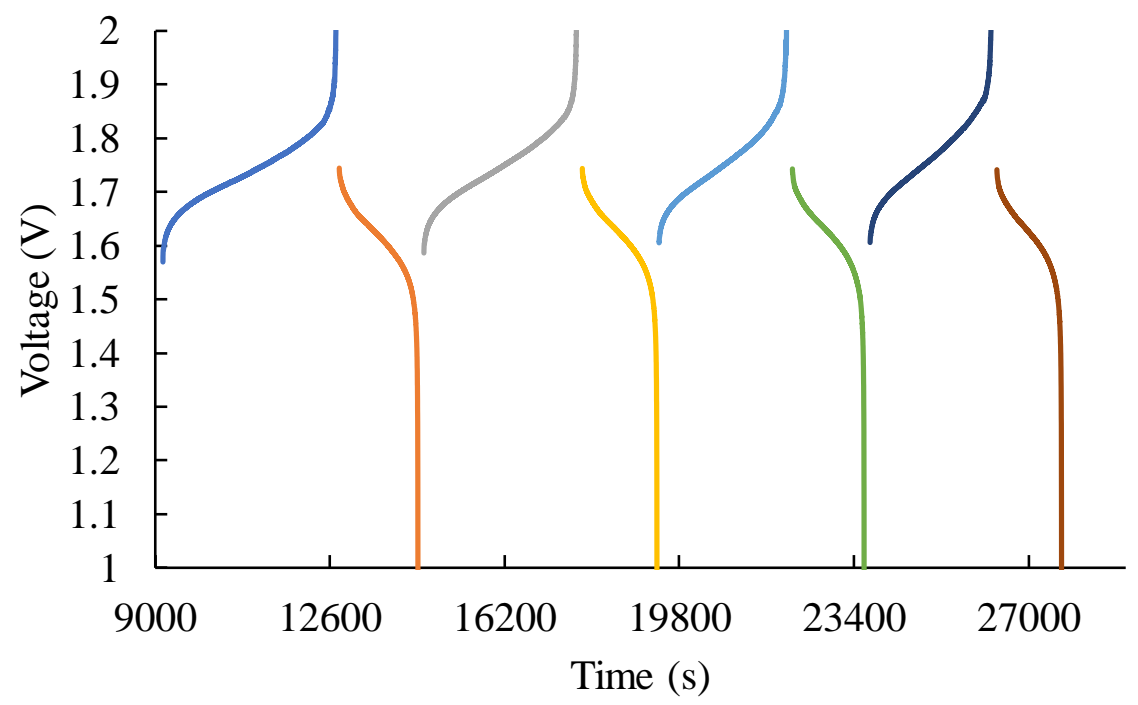

Figure 15. Charge/discharge cycles for the RFB using vanadium as the anolyte and manganese as the catholyte.

The coulombic, voltage, and energy efficiency for the RFB using vanadium as the anolyte and manganese as the catholyte are shown in Figure 16. The average coulombic efficiency was $51.04 \%$, the average voltage efficiency was $92.08 \%$, and the average 
energy efficiency was $46.99 \%$. The efficiencies are relatively stable over four cycles; however, the coulombic efficiency is low. Like capacity fade, this is a result of active species crossover and precipitation of $\mathrm{MnO}_{2}$. The voltage efficiency is reasonably high indicating the system has enough ionic and electrical conductivity.

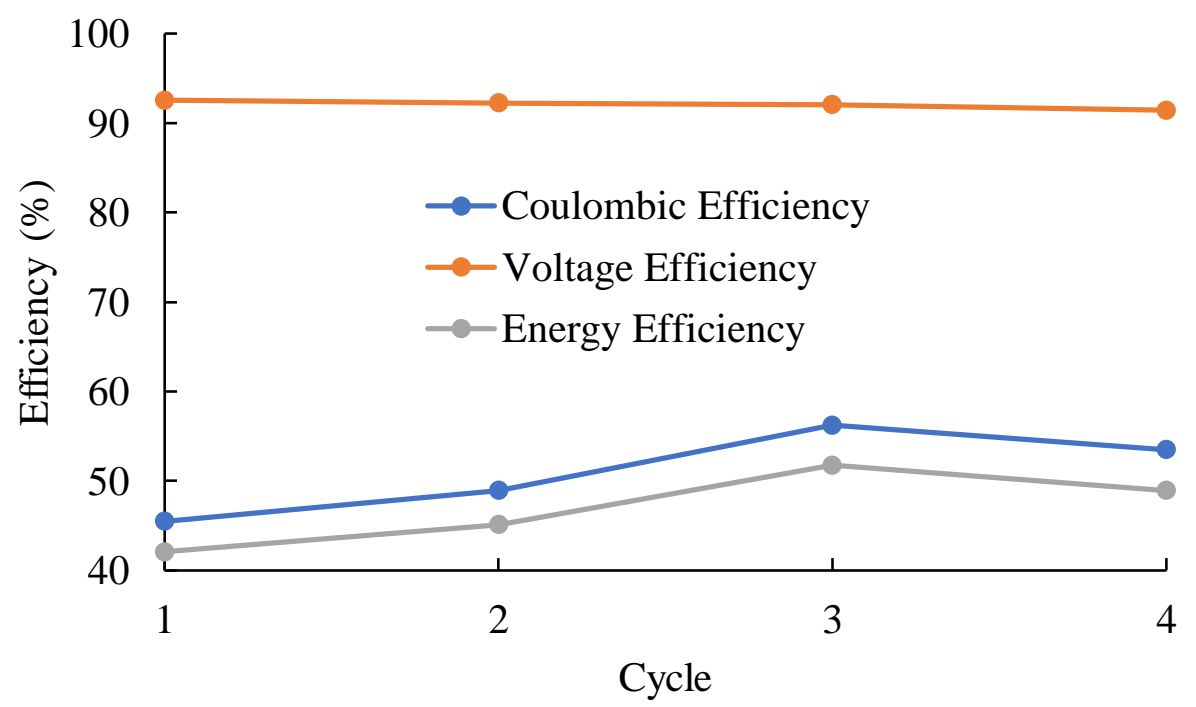

Figure 16. Coulombic, voltage, and energy efficiency for an RFB using vanadium as the anolyte and manganese as the catholyte.

The vanadium anolyte and manganese catholyte, with the same composition as before, was tested again with a current density of $50 \mathrm{~mA} \mathrm{~cm}{ }^{-2}$. Increasing the current density can increase the coulombic efficiency by allowing less time for crossover to occur. Figure 17 shows a 5-cycle charge/discharge test. Capacity loss is seen by the reduced charge and discharge duration as the cycle numbers increase. The average charge capacity was $33.6 \mathrm{mAh}$ and the average discharge capacity was $27.1 \mathrm{mAh}$. This test was performed using the same volume of electrolyte as the previous test. The capacity was already reduced 
after the test cycles shown in Figure 15. Additionally, running at a higher current density results in reduced charge capacity as the cutoff conditions are met before the SOC reaches the same state as a lower current density. The discharge power density was $73 \mathrm{~mW} \mathrm{~cm} \mathrm{c}^{-2}$; over double the previous test, as expected.

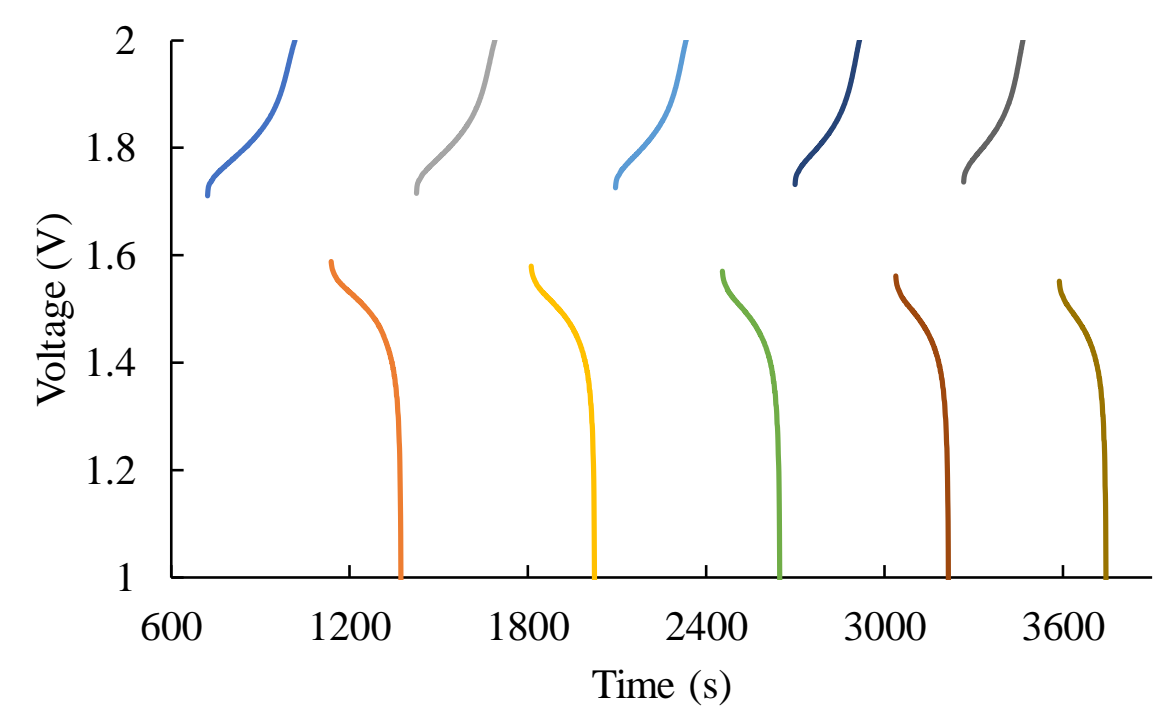

Figure 17. Charge/discharge cycles for an RFB using vanadium as the anolyte and manganese as the catholyte with a charge/discharge current density of $50 \mathrm{~mA} \mathrm{~cm}$.

The coulombic, voltage, and energy efficiency of the vanadium anolyte and manganese catholyte test at $50 \mathrm{~mA} \mathrm{~cm}^{-2}$ are shown in Figure 18. The average coulombic efficiency over the 5 cycles was $80.35 \%$, the average voltage efficiency $79.58 \%$, and the average energy efficiency $63.95 \%$. The efficiencies remain relatively constant over the 5 cycles. Compared to the previous test at $20 \mathrm{~mA} \mathrm{~cm}^{-2}$, the coulombic efficiency increased significantly while the voltage efficiency dropped significantly. The energy efficiency increased by $\sim 17 \%$. The decrease in voltage efficiency is a direct result of increased current 
density. As voltage efficiency is affected by resistance in the cell components, an increase in current intrinsically results in a decrease in voltage efficiency. The gradual decline of voltage efficiency as the cycle number increases are attributed to coating of the graphite felt with the precipitate $\mathrm{MnO}_{2}$, resulting in increased ohmic losses.

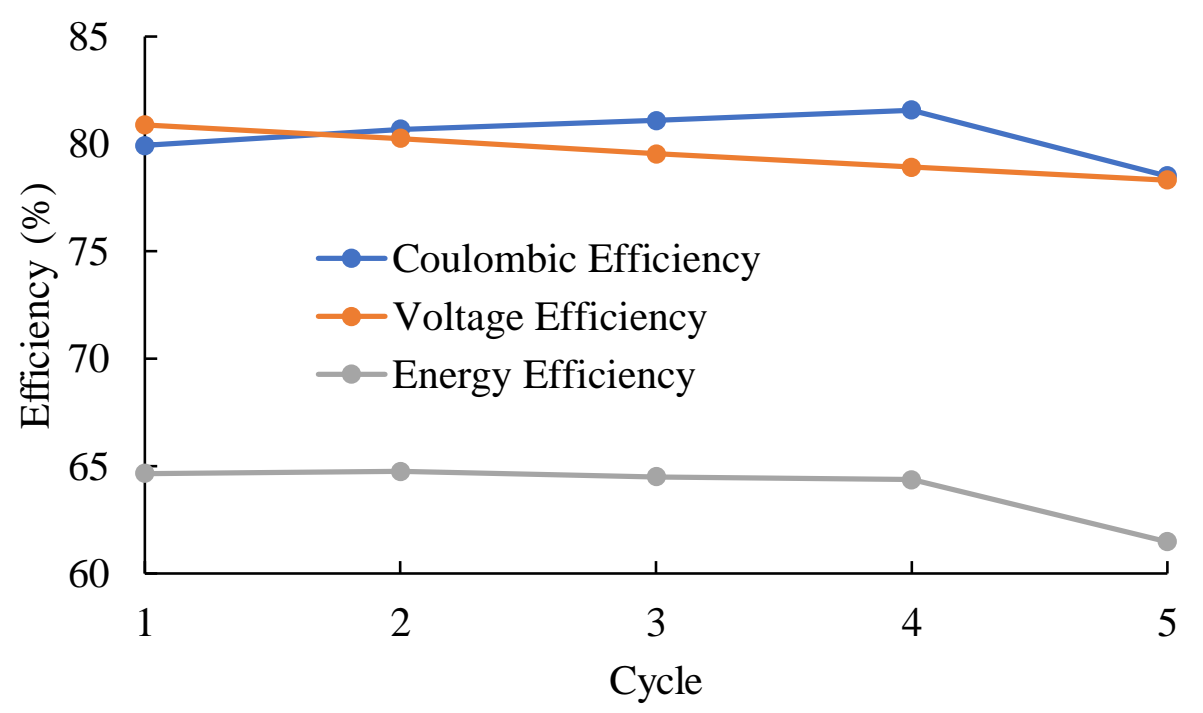

Figure 18. Coulombic, voltage, and energy efficiency for an RFB using vanadium as the anolyte and manganese as the catholyte with a charge/discharge current density of

$$
50 \mathrm{~mA} \mathrm{~cm}^{-2} \text {. }
$$

\subsubsection{Zinc/manganese}

Testing of the zinc and manganese redox couples together is a step into the unknown as these redox couples have never been tested together. The zinc redox couple is

$$
\mathrm{Zn}^{2+}+e^{-} \leftrightarrow \mathrm{Zn} \quad-0.7618 \mathrm{~V}(\text { vs. SHE) (9) }
$$


The overall theoretical potential of this system is $2.303 \mathrm{~V}$. There is no published literature on this system together; however, publications exist on each separately with a large amount of information on the zinc anolyte. Zinc and manganese are cheap compared to vanadium and with such a high theoretical potential, this system could be substantially better than the all-vanadium system in terms of cost, energy density, and power density.

The same catholyte composition as previous testing was used, $0.5 \mathrm{M} \mathrm{MnSO}_{4}$ in $3 \mathrm{M} \mathrm{H}_{2} \mathrm{SO}_{4}$. The anolyte composition was $0.5 \mathrm{M} \mathrm{ZnSO}_{4}$ in $3 \mathrm{M} \mathrm{H}_{2} \mathrm{SO}_{4}$. The electrodes and ion-exchange membrane were the same as previously used, graphite felt and Nafion, respectively. The current density used for charge/discharge testing was $20 \mathrm{~mA} \mathrm{~cm}^{-2}$. The charge/discharge cycle results are shown in Figure 19. It is obvious from the charge/discharge curve durations that the discharge capacity, $10.08 \mathrm{mAh}$, was not close to the charge capacity, $33.11 \mathrm{mAh}$. The cell was taken apart after cycling and no zinc was observable on the graphite felt electrode through an optical microscope. The low discharge capacity is due to poor plating of the zinc on the graphite felt. The discharge power density was $39.2 \mathrm{~mW} \mathrm{~cm}$, which is higher than the vanadium and manganese system at the same current density, due to the high discharge potential. At the onset of discharge the potential difference of the cell was above $2.1 \mathrm{~V}$, in close agreement with the anticipated potential difference based on standard redox potentials. With controlled plating and good kinetics, this RFB could exhibit a very high energy density. 


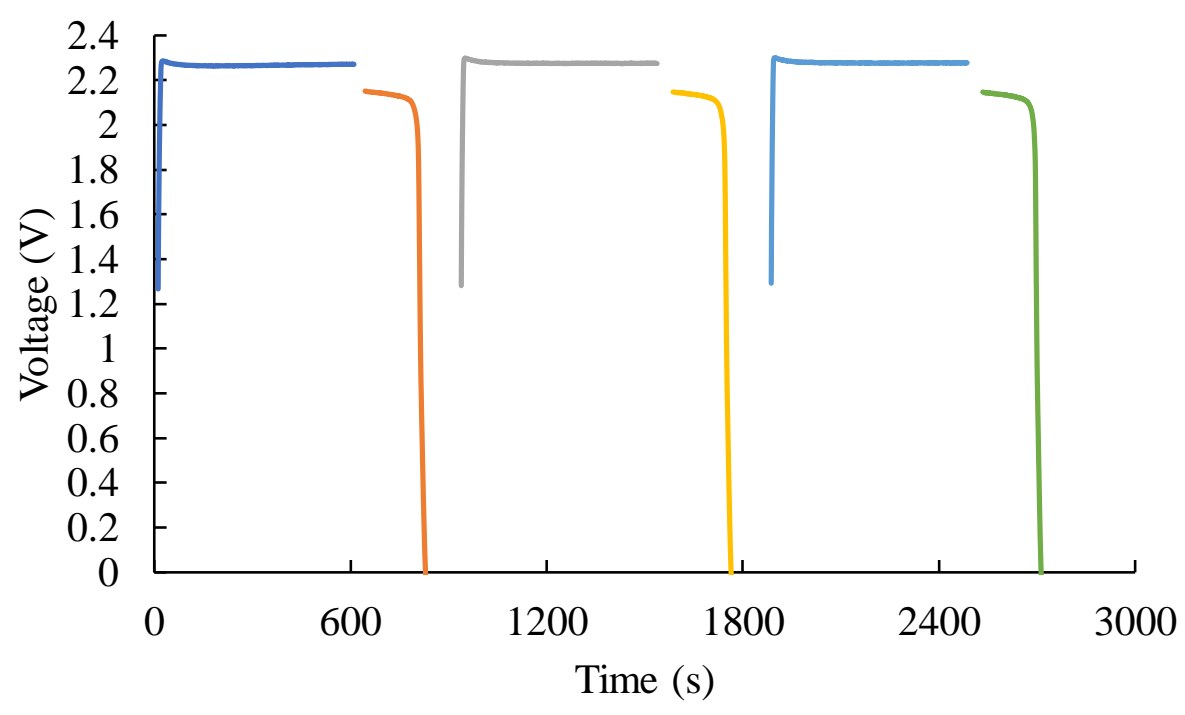

Figure 19. Charge/discharge cycles for an RFB using zinc as the anolyte and manganese as the catholyte with a charge/discharge current density of $20 \mathrm{~mA} \mathrm{~cm}^{-2}$.

The coulombic, voltage, and energy efficiency are shown in Figure 20. The voltage efficiency was a respectable $86.26 \%$; ionic and electrical conductivity are not a large issue. The coulombic efficiency was $30.16 \%$ resulting in an energy efficiency of $26.02 \%$. This is attributed to the poor plating of zinc onto the graphite felt electrode, which means that little zinc is available to take place in discharge reactions. 


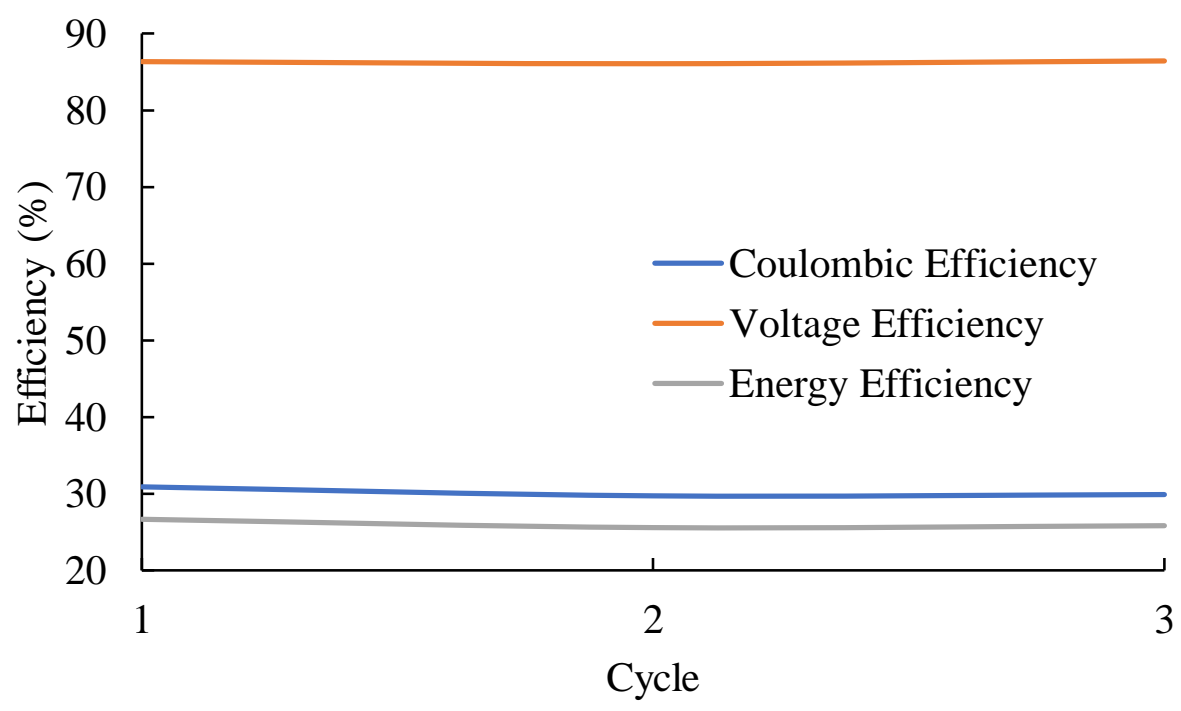

Figure 20. Coulombic, voltage, and energy efficiency for an RFB using zinc as the anolyte and manganese as the catholyte with a charge/discharge current density of

$$
20 \mathrm{~mA} \mathrm{~cm}^{-2} \text {. }
$$

After testing, the vanadium/manganese and zinc/manganese cells were taken apart and the graphite felt was examined using a SEM. Figure 21(a) shows a sample of untested graphite felt. The graphite in graphite felt is structured into fine uniform hairs randomly intertwined throughout the sample with little impurities visible. Figure 21(b) shows the anode of the vanadium/manganese RFB. The vanadium anolyte does not have any chemical reaction avenues to precipitate so, the particles present are a result of manganese ion crossover. After crossover, some of the manganese ions that reached the electrode surface participated in electrochemical reactions until forming the $\mathrm{MnO}_{2}$ precipitate. Figure 21(c) shows the cathode of the zinc and manganese RFB. The precipitated $\mathrm{MnO}_{2}$ has attached to and agglomerated on the hairs of the graphite felt. This is the source of the capacity and coulombic efficiency loss. Figure 21(d) is the same cathode but, magnified further. From 
this image, it is clear the coating of precipitate is thick and will reduce the number of reaction sites on the carbon felt.

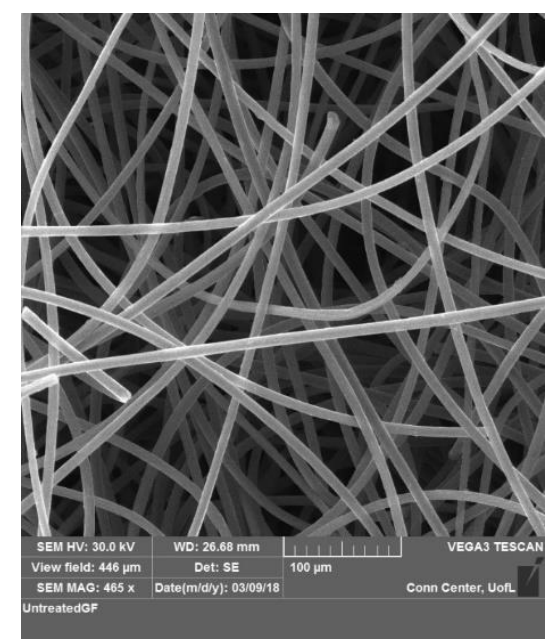

(a)

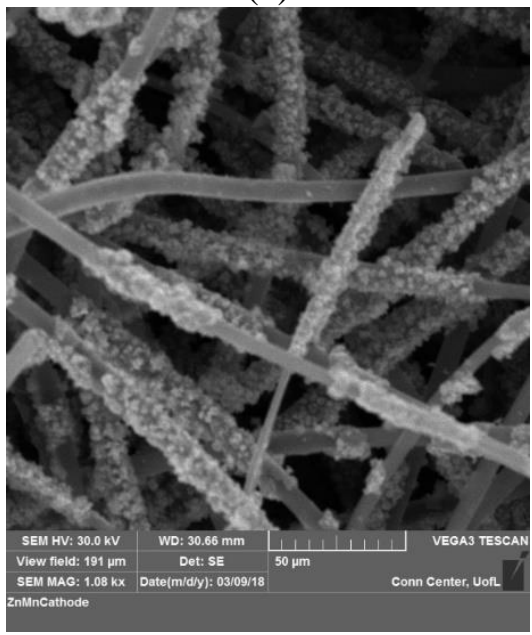

(c)

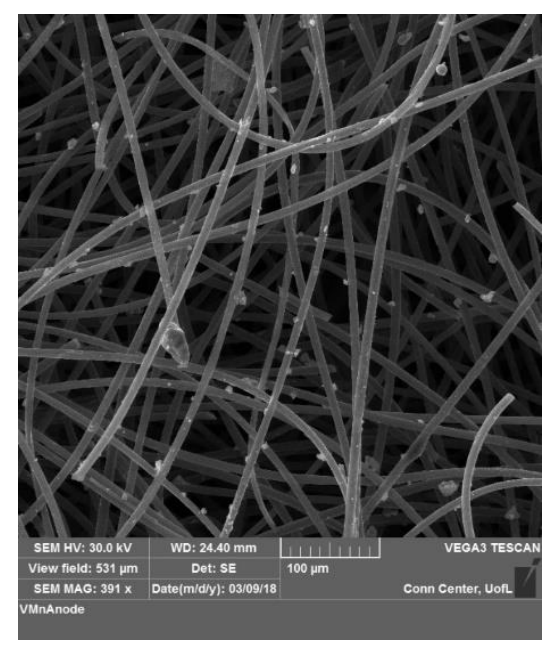

(b)

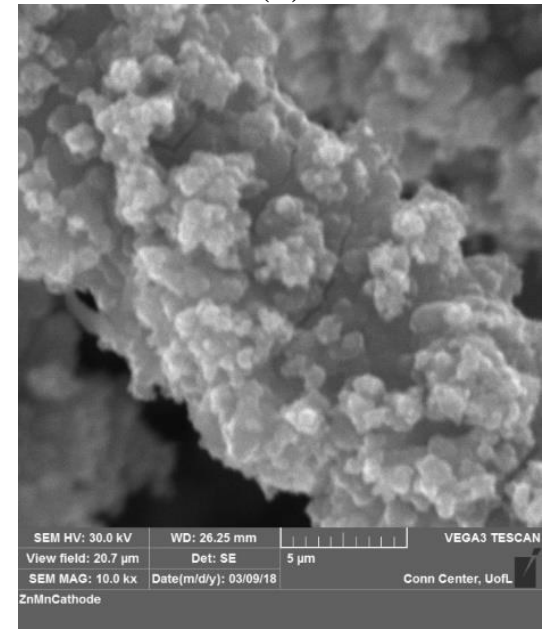

(d)

Figure 21. SEM images of graphite felt (a) before testing, (b) after testing of the anode in the vanadium manganese RFB, (c) after testing of the cathode in the zinc/manganese RFB, and (d) higher magnification after testing of the cathode in the zinc/manganese RFB. 
Additional testing and optimization of the all-vanadium RFB and zinc redox couple is outside of the scope of this research. Although, great results were obtained with the allvanadium RFB. It is desired with this study to push the envelope of RFB research. The zinc anolyte is promising due to its low cost and low potential; however, this is a plating reaction. Plating reactions remove the fundamental advantage of RFB by reconnecting energy and power. Recent research has shown that the separation of energy and power in plating reactions can be achieved by introducing a conductive slurry into the electrolyte and plating onto that slurry [75]. The conductive slurry is flowable so, the charged active species can flow out of the electrochemical cell and into storage tanks. 


\section{CHAPTER 4}

\section{THE $\mathrm{Mn}^{2+} / \mathrm{Mn}^{3+}$ AND Ce $\mathrm{e}^{3+} / \mathrm{Ce}^{4+}$ REDOX COUPLES AS CATHOLYTES FOR RFBs}

\subsection{Background and state-of-the-art}

The use of the manganese redox couple, $\mathrm{Mn}^{2+} / \mathrm{Mn}^{3+}$, has been proposed recently and tested in both aqueous and non-aqueous electrolyte solutions. The manganese redox couple chemical reaction and standard electrode potential is

$$
\mathrm{Mn}^{3+}+e^{-} \leftrightarrow \mathrm{Mn}^{2+}
$$

where this redox couple is used in the catholyte of an aqueous RFB [96]. A primary issue with the manganese redox couple is the spontaneous disproportionation of $\mathrm{Mn}^{3+}$ to the precipitate $\mathrm{MnO}_{2}$ according to the following reaction

$$
2 \mathrm{Mn}^{3+}+2 \mathrm{H}_{2} \mathrm{O} \leftrightarrow \mathrm{Mn}^{2+}+\mathrm{MnO}_{2}+4 \mathrm{H}^{+}
$$

Kaku et al. studied the effect of the $\mathrm{Ti}^{4+}$ ion on the manganese redox couple [51]. They found that by mixing $\mathrm{Ti}^{4+}$ with the manganese redox couple, disproportionation of $\mathrm{Mn}^{3+}$ to $\mathrm{MnO}_{2}$ was suppressed and the morphology of $\mathrm{MnO}_{2}$ improved by maintaining a small particle size that has the ability to flow through the battery without clogging, and reduce back to $\mathrm{Mn}^{2+}$ on the electrode surface [51]. In this study, the $\mathrm{Ti}^{4+} / \mathrm{Ti}^{3+}$ redox couple 
was used as the anolyte. The anolyte and catholyte were combined, forming a mixed electrolyte. The use of a mixed electrolyte has the benefit of reducing crossover effects by decreasing the concentration gradient across the ion-exchange membrane [51]. The drawback to using a mixed electrolyte is a possible decrease in solubility of the active species.

To study the effects of $\mathrm{Ti}^{4+}$ on the disproportionation of $\mathrm{Mn}^{3+}$, the samples of $\mathrm{Kaku}$ et al. contained varying concentrations of titanium $(0,0.25,0.5,0.75$, and $1 \mathrm{M})$ with a fixed concentration of manganese and sulfuric acid, $1 \mathrm{M}$ and $3 \mathrm{M}$, respectively [51]. Each sample was charged to a 50 and $90 \%$ SOC and then stored for 1 week at room temperature. The samples were analyzed by inductively coupled plasma atomic emission spectroscopy (ICP-OES), scanning electron microscopy (SEM) with energy dispersive X-ray spectroscopy (EDX), and X-ray diffraction (XRD). After one week, the precipitate in the stored sample was separated and the SOC of the supernate was obtained by ICP-OES. Comparing this with the original SOC allows for the calculation of $\mathrm{MnO}_{2}$ which has precipitated. With a high $\mathrm{Ti}^{4+}$ concentration and $50 \% \mathrm{SOC}$, the amount of precipitate decreased significantly, close to zero with $1 \mathrm{M}$ titanium [51]. With a $90 \%$ SOC, the decrease in $\mathrm{MnO}_{2}$ precipitation was less dramatic [51]. Kaku et al. found that increasing the $\mathrm{Ti}^{4+}$ concentration, at $90 \% \mathrm{SOC}$, lead to a decrease in $\mathrm{MnO}_{2}$ particle size, from over 1 $\mu \mathrm{m}$ at low titanium concentration to less than $100 \mathrm{~nm}$ at high titanium concentration, shown in Figure 22(a and b) [51]. 


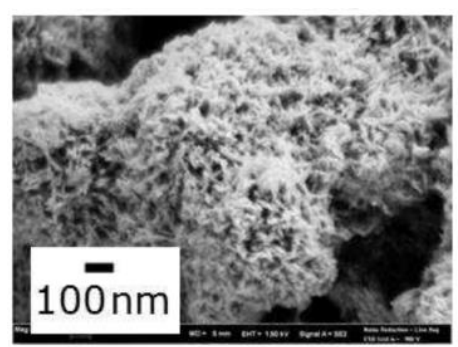

(a)

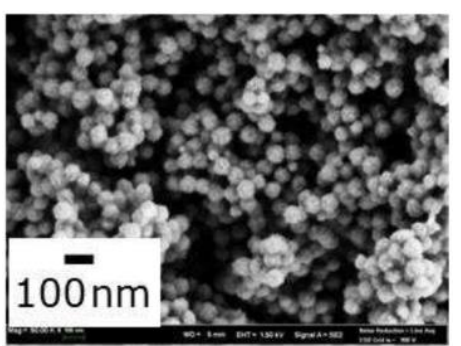

(b)

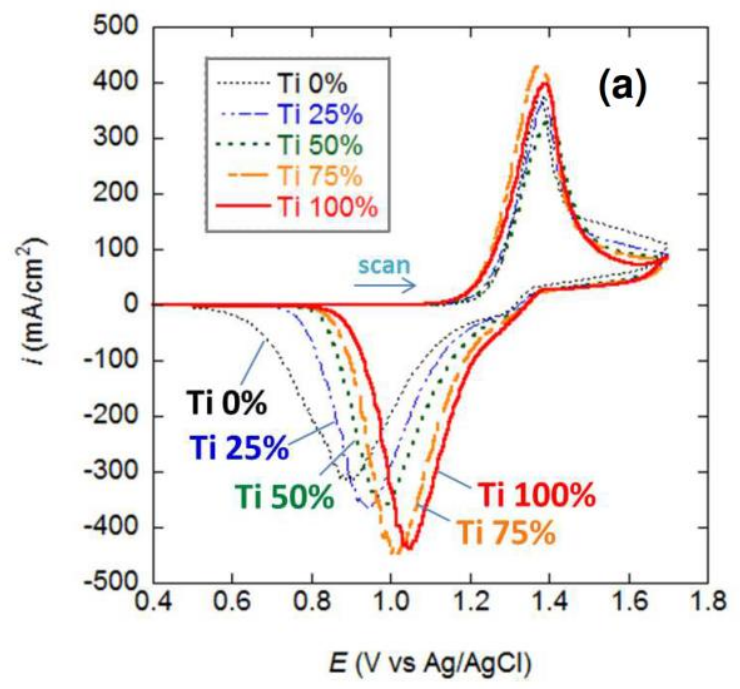

(c)

Figure 22. Morphology of $\mathrm{MnO}_{2}$ precipitates after charging to $90 \% \mathrm{SOC}$ in a $1 \mathrm{M}$ manganese and $3 \mathrm{M}$ sulfuric acid solution with (a) $0 \mathrm{M}$ titanium and (b) $1 \mathrm{M}$ titanium; (c) cyclic voltammetry of a $1 \mathrm{M}$ manganese and $3 \mathrm{M}$ sulfuric acid solution with a varying concentration of titanium (Ti $100 \%$ being $1 \mathrm{M}$ titanium) [51].

With a high concentration of titanium, the main phase found was gamma- $\mathrm{MnO}_{2}$, which is more reactive compared to alpha- $\mathrm{MnO}_{2}$, found at lower titanium concentrations [51]. It was determined that the presence of $\mathrm{Ti}^{4+}$ ions affect the crystal structure of $\mathrm{MnO}_{2}$ resulting in a more reactive phase and lower crystallinity [51]. UV-visible spectroscopy (UV-vis) showed that in the mixed electrolyte, the $\mathrm{Ti}^{4+}$ ion makes a complex with the $\mathrm{Mn}^{3+}$ 
ion [51]. Figure 22(c) shows a decrease in peak separation and asymmetry of the CV curves with increased titanium content.

Dong et al. explored the use of a mixed $\mathrm{Ti}^{4+}$ and $\mathrm{Mn}^{2+}$ aqueous electrolyte in a RFB $[22 ; 23]$. The titanium redox couple chemical reaction and standard electrode potential is

$$
\mathrm{TiO}^{2+}+2 \mathrm{H}^{+}+e^{-} \leftrightarrow \mathrm{Ti}^{3+}+\mathrm{H}_{2} \mathrm{O} \quad 0.1 \mathrm{~V}(\text { vs. SHE) (12) }
$$

where the standard theoretical potential of the titanium and manganese redox reactions is $1.41 \mathrm{~V}$; high compared to other aqueous systems [22; 23]. Dong et al. prepared the mixed electrolyte using up to $1 \mathrm{M} \mathrm{MnSO}_{4}$, up to $1.5 \mathrm{M} \mathrm{TiOSO}_{4}$, and $3 \mathrm{M} \mathrm{H}_{2} \mathrm{SO}_{4}$ [22; 23]. The cell used for electrochemical testing had an electrode area of $0.785 \mathrm{~cm}^{2}$ and used carbon felt for both the anode and cathode $[22 ; 23]$. For the manganese only electrolyte, potential varies with SOC and peaks at a SOC of $40 \%$, after which disproportionation to $\mathrm{MnO} 2$ decreases the concentration of $\mathrm{Mn}^{3+}$ resulting in a lower potential $[22 ; 23]$. With the mixed electrolyte, potential continues to increase up to $90 \%$ SOC, indicating the suppression of $\mathrm{MnO}_{2}$ precipitation $[22 ; 23]$. A flow cell using graphite felt with an active area of $9 \mathrm{~cm}^{2}$ was used for charge/discharge testing, Figure 23(a) [22; 23]. 


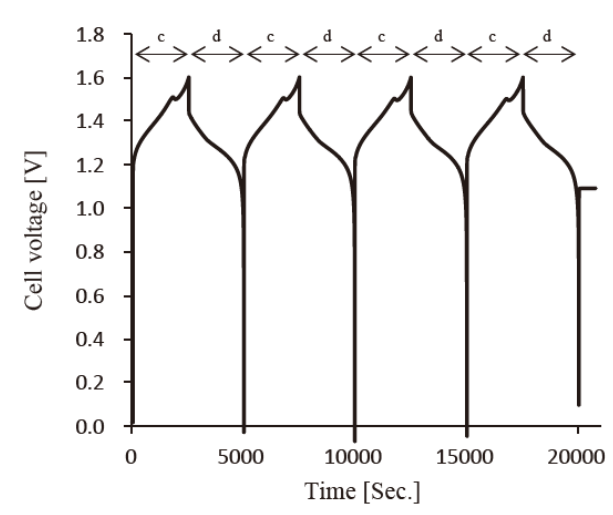

(a)

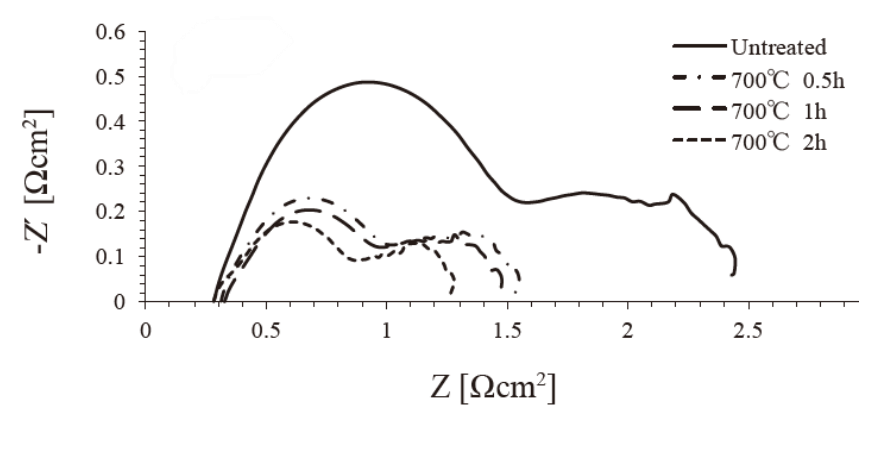

(b)

Figure 23. (a) Charge/discharge testing of a full RFB using a mixed titanium and manganese electrolyte [22]. (b) EIS of a symmetric cell [22].

The cell exhibited an energy efficiency of $88.7 \%$ with a coulombic efficiency of $99.5 \%$ and a voltage efficiency of $89.2 \%$ at a current density of $50 \mathrm{~mA} \mathrm{~cm}{ }^{-2}[22 ; 23]$. Carbon corrosion was observed at the cathode $[22 ; 23]$. To improve durability of the electrode, high-temperature oxidation resistant carbon paper was used [22; 23]. The carbon paper was pretreated by heat treating it in air at $700{ }^{\circ} \mathrm{C}$ for 0.5 to 2 hours $[22 ; 23]$. Dong et al. also used electrochemical impedance spectroscopy (EIS) with a symmetric cell to test the internal resistance when using the mixed electrolyte with different electrodes, Figure 23(b) [23]. The EIS response indicated heat treatment reduces charge transfer resistance. This is believed to be a result of improved wettability [23]. Power density reached a maximum of $357 \mathrm{~mW} \mathrm{~cm}^{-2}$ at $50 \% \mathrm{SOC}$ [22; 23]. At high SOC the concentration of $\mathrm{Mn}^{3+}$ is increased resulting in increased precipitation of $\mathrm{MnO}_{2}$, which disturbs reactant transport [23]. Using carbon felt, the power density increased to $640 \mathrm{~mW} \mathrm{~cm}{ }^{-2}$; however, significant oxidation and corrosion of the electrode occurred [23]. With carbon paper as the cathode and carbon felt as the anode, Dong et al. were able to achieve an energy 
efficiency of $83.2 \%$, coulombic and voltage efficiency of 99.8 and $83.4 \%$, respectively, at a current density of $100 \mathrm{~mA} \mathrm{~cm}^{-2}$ [23].

Hong et al. studied the use of the manganese redox couple $\left(\mathrm{Mn}^{2+} / \mathrm{Mn}^{3+}\right)$ as the catholyte and the vanadium redox couple $\left(\mathrm{V}^{2+} / \mathrm{V}^{3+}\right)$ as the anolyte [41]. The standard theoretical potential of theses redox couples is $1.77 \mathrm{~V}$, high compared to other aqueous systems (all-vanadium 1.26 V, iron-chromium $1.18 \mathrm{~V}$ ) [41]. The electrolyte used for charge/discharge testing was composed of $1.2 \mathrm{M} \mathrm{Mn}^{2+}$ in $3 \mathrm{M} \mathrm{H}_{2} \mathrm{SO}_{4}$ as the catholyte and $1.2 \mathrm{M} \mathrm{V}^{3+}$ in $3 \mathrm{M} \mathrm{H}_{2} \mathrm{SO}_{4}$ as the anolyte [41]. The flow battery cell employed polyacrylonitrile (PAN) based carbon felt electrodes with an electrode area of $8 \mathrm{~cm}^{2}$ [41]. The electrodes were pretreated by immersion in sulfuric acid for $5 \mathrm{~h}$, washed to neutral $\mathrm{pH}$, and then heat treated for $2 \mathrm{~h}$ at $450{ }^{\circ} \mathrm{C}$ [41]. Nafion 117 was used as the membrane and the electrolyte flow rate was $6 \mathrm{~mL} \min ^{-1}$ [41]. During CV with a SCE reference electrode, an oxidation peak was observed at $1.29 \mathrm{~V}$ and two reduction peaks at 1.11 and $0.76 \mathrm{~V}$ [41]. It was found, by using a transient potential-step experiment and the Butler-Volmer equation, that the reduction peak at $1.11 \mathrm{~V}$ is associated with reduction of $\mathrm{Mn}^{3+}$ to $\mathrm{Mn}^{2+}$ and the reduction peak at $0.76 \mathrm{~V}$ is associated with disproportionation of $\mathrm{MnO}_{2}$ to $\mathrm{Mn}^{2+}$ [41].

The diffusion coefficient of $\mathrm{Mn}^{2+}$ was found by Hong et al. to be $0.236 \times 10^{-6} \mathrm{~cm}^{2} \mathrm{~s}^{-1}$ using the Levich equation

$$
i_{d}=0.0620 n F A D^{2 / 3} v^{-1 / 6} C \omega^{1 / 2}
$$

where $i_{D}$ is the limiting current density, $n$ is the number of electrons transferred, $F$ is Faraday's constant, $A$ is the electrode area, $D$ is the diffusion coefficient, $v$ is the kinematic 
viscosity, $C$ is the active species concentration, and $\omega$ is the angular rotation rate of a rotating disc electrode (RDE) [41]. Figure 24(a) shows the linear polarization of a manganese electrolyte at different rotation rates. The limiting currents at different potentials were plotted against the square root of angular velocity, shown in Figure 24(b). Using EIS, Hong et al. determined that a $3 \mathrm{M}$ sulfuric acid concentration was optimum as a higher concentration results in mass transport difficulties due to an increase in viscosity [41]. A degradation in the cycle performance became apparent after 8 cycles; however, the average discharge voltage was $1.66 \mathrm{~V}$, substantially higher than the allvanadium RFB [41]. Hong et al. achieved an energy efficiency of $81.2 \%$ at $80 \mathrm{~mA} \mathrm{~cm}-2$ with a coulombic efficiency of $92.5 \%$ and a voltage efficiency of $85.3 \%$ [41].

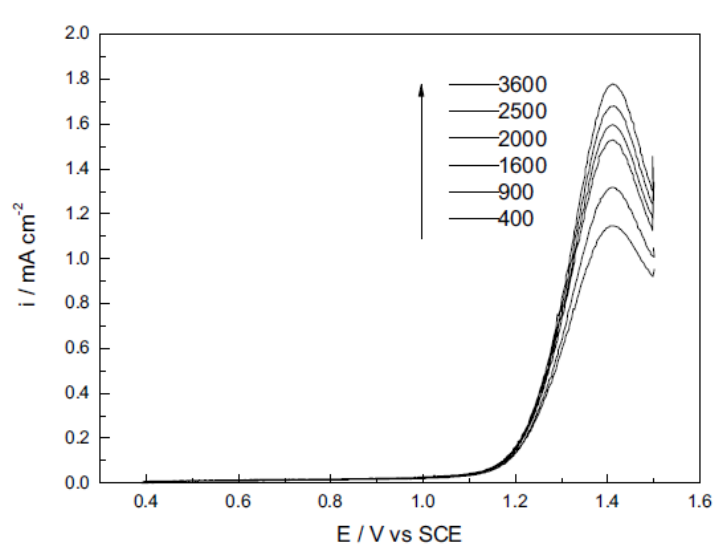

(a)

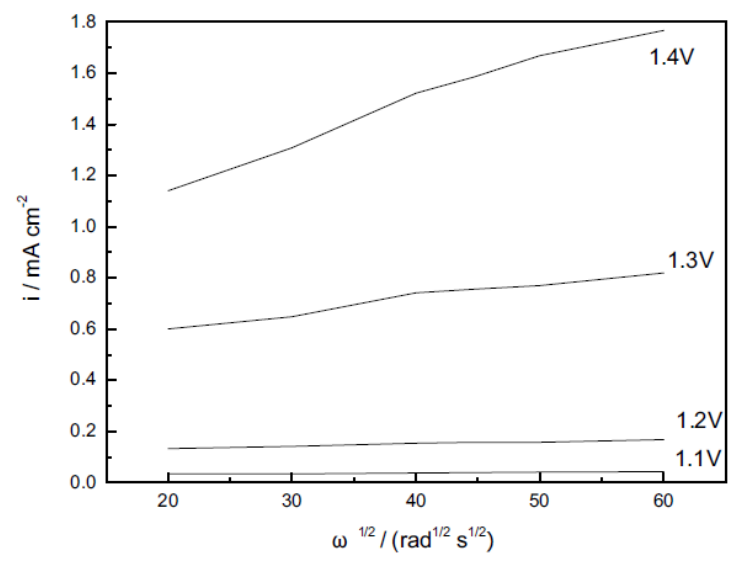

(b)

Figure 24. (a) Linear polarization of a manganese electrolyte at different rotation rates

[41]. (b) Limiting current at different potentials plotted against the square root of the angular velocity for a manganese electrolyte [41].

Xue et al. studied the $\mathrm{Mn}^{2+} / \mathrm{Mn}^{3+}$ redox couple to explore a suitable electrode material, supporting electrolyte, and the electrochemical reaction mechanism [109]. 
Polyacrylonitrile (PAN) based carbon felt and spectral pure graphite were used as the electrode for testing [109]. Xue et al. found that when running charge/discharge cycles with spectral pure graphite, no deposition of $\mathrm{MnO}_{2}$ occurred; however, with carbon felt, some deposition was found and tested using XRD to confirm it was $\mathrm{MnO}_{2}$ [109]. During CV an oxidation peak was seen at $1.29 \mathrm{~V}$ vs. a saturated calomel electrode (SCE) and found to correspond with the oxidation of $\mathrm{Mn}^{2+}$ to $\mathrm{Mn}^{3+}$ [109]. Two reduction peaks were seen at $1.11 \mathrm{~V}$ and $0.76 \mathrm{~V}$ and found to correspond to the reduction of $\mathrm{Mn}^{3+}$ to $\mathrm{Mn}^{2+}$ and disproportionation of $\mathrm{MnO}_{2}$ to $\mathrm{Mn}^{2+}$, respectively [109]. At $5 \mathrm{M}$ sulfuric acid, the ratio of peak current was about 1.036, showing high reversibility [109]. With an increase in sulfuric acid concentration, the solubility of manganous sulfate quickly decreased to $0.17 \mathrm{M}$ at $6 \mathrm{M}$ sulfuric acid [109]. Such a low active species concentration severely limits energy density and is not practical. Using EIS, at $5 \mathrm{M}$ sulfuric acid the electrolyte resistance was found to be $0.240 \Omega$ and the charge transfer resistance was found to be $0.980 \Omega$ [109]. Xue et al. demonstrated an energy efficiency of $62.7 \%$ with a current density of $20 \mathrm{~mA} \mathrm{~cm}^{-2}$ [109]. The coulombic efficiency was $69.4 \%$ and the voltage efficiency was $90.4 \%$ with an average discharge voltage of $1.66 \mathrm{~V}$ [109].

Sleightholme et al. developed a non-aqueous flow battery using 0.05 M manganese acetylacetonate, with the $\mathrm{Mn}^{2+} / \mathrm{Mn}^{3+}$ and $\mathrm{Mn}^{3+} / \mathrm{Mn}^{4+}$ redox couples, in acetonitrile with 0.5 M tetraethylammonium tetrafluoroborate as the supporting electrolyte [89]. The coulombic efficiency of this flow battery was $74 \%$ after 3 cycles with an energy efficiency of $21 \%$ due to a low voltage efficiency [89]. The low voltage efficiency was contributed to the large electrode separation in the $\mathrm{H}-\mathrm{cell}, 10 \mathrm{~cm}$, and the use of an anion-exchange membrane which results in significant ohmic overpotentials [89]. Chen et al. developed a 
manganese-hydrogen hybrid battery using the $\mathrm{Mn}^{2+} / \mathrm{MnO}_{2}$ redox couple at the cathode [15]. The battery exhibited a discharge voltage of $1.3 \mathrm{~V}$ at a current density of $100 \mathrm{~mA} \mathrm{~cm}{ }^{-2}$ with a lifetime of more than 10,000 cycles without decay [15]. With a $4 \mathrm{M} \mathrm{Mn}^{2+}$ solution, the energy density was found to be $210 \mathrm{Wh} \mathrm{L}^{-1}$ [15]. Using $1 \mathrm{M} \mathrm{MnSO}_{4}$ with $0.05 \mathrm{M} \mathrm{H}_{2} \mathrm{SO}_{4}$, an energy efficiency of $71 \%$ was obtained [15]. XRD of the $\mathrm{MnO}_{2}$ coated carbon felt confirmed the gamma phase [15]. Chen et al. fabricated a cylindrical cell to demonstrate scale-up of the Mn-H battery, shown in Figure 25. The cylindrical cell was composed of a cylindrical steel vessel lined with a layer of carbon felt as the cathode with a platinum coated carbon felt anode at the center of the vessel [15]. The chamber was filled with the $\mathrm{Mn}^{2+}$ electrolyte; no membrane was utilized [15]. The cylindrical cell achieved a coulombic efficiency of $96.7 \%$ after 50 cycles and retained $94.2 \%$ of its charge capacity after 1,400 cycles [15].
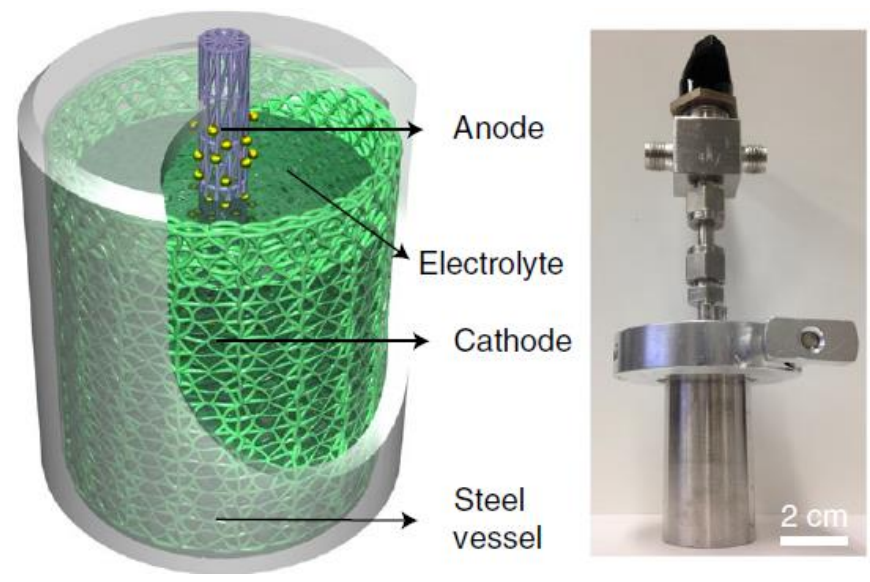

Figure 25. Schematic and photograph of cylindrical cell used to operate a manganesehydrogen hybrid battery. 
Pan et al. demonstrated a non-flowing zinc/manganese battery using the $\mathrm{Zn} / \mathrm{Zn}^{2+}$ redox couple combined with a $\alpha-\mathrm{MnO}_{2}$ nanofiber cathode and zinc metal anode [72]. The system exhibited a capacity retention of $92 \%$ after 5,000 cycles [72]. The prepared $\mathrm{MnO}_{2}$ cathode exhibited a highly crystalline structure seen using a high-resolution transmission electron microscopy (HRTEM) [72]. After the first discharge cycle, the micrometer-long nanofibers changed to short nanorods and nanoparticle aggregates [72]. This was found to be beneficial as it enhanced the structural mechanics and kinetics of the cathode by releasing strain and reducing diffusion distance [72]. Using TEM and XRD, Pan et al. found that, instead of $\mathrm{Zn}^{2+}$ intercalation, $\mathrm{MnO}_{2}$ reacts with water to form $\mathrm{MnOOH}$ and $\mathrm{ZnO}_{2}$ reacts with water to form large flake-like $\mathrm{ZnSO}_{4}\left[\mathrm{Zn}(\mathrm{OH})_{2}\right]_{3}[72]$.

The difficulties in using the $\mathrm{Mn}^{2+} / \mathrm{Mn}^{3+}$ redox couple in a RFB is fouling of the electrode, gas evolution, active species crossover, and spontaneous precipitation of $\mathrm{MnO}_{2}$ that comes from the charged manganese active species $\mathrm{Mn}^{3+}$. The $\mathrm{MnO}_{2}$ precipitate results in reduced capacity of the catholyte, a passivating layer on the electrode surface, and potential clogging of the fluid flow system. Currently, only a handful of studies have been published which report the use of manganese as the cathode redox couple in a RFB $[23 ; 41 ; 51 ; 109]$. Some work has been done to alleviate issues resulting from the precipitation of $\mathrm{MnO}_{2}$, with promising results; however, still little is known about the mechanism of this reaction. The higher potential of manganese results in significant amounts of oxygen evolution and fouling of the standard RFB electrode, graphite, compared to the vanadium catholyte.

The $\mathrm{Ce}^{3+} / \mathrm{Ce}^{4+}$ redox couple shows promise in RFB due to its good reversibility, high kinetics, high solubility in some solvents, high potential, and cost compared to 
vanadium. Upon its inception, platinum-based electrodes were often used for the $\mathrm{Ce}^{3+} / \mathrm{Ce}^{4+}$ redox couple because they allow high current densities without degrading. The cost of platinum-based electrodes; however, is prohibitive. The challenge in selecting an electrode for cerium is activity of the redox couple on its surface, degradation due to corrosion at high potentials, activity toward hydrogen evolution, and cost. Several different electrode materials were tested for use in a Zn/Ce RFB including different graphite felt modifications and platinized titanium mesh [55]. Leung et al. used methanesulfonic acid as a solvent due to the high solubility of cerium ions. Using a platinized titanium mesh electrode, an energy efficiency of $43 \%$ was obtained at a current density of $50 \mathrm{~mA} \mathrm{~cm}^{-2}$ [55]. With a carbon felt electrode the energy efficiency was $63 \%$ at $50 \mathrm{~mA} \mathrm{~cm}^{-2}$ [55]. Leung et al. found no evidence of corrosion using the platinized titanium mesh; however, corrosion was observed on the graphite electrode and carbon particles were observed in the electrolyte solutions after operation [55].

The addition of chloride ions in the methansulfonate anolyte solution was explored by Amini et al. [3]. The electrodes utilized in this iteration of the $\mathrm{Zn} / \mathrm{Ce} \mathrm{RFB}$ were platinized titanium mesh as the cathode and a copper current collector, on which $\mathrm{Zn}$ is deposited, as the anode. The addition of chloride ions increased energy efficiency from 38.8 to $51.9 \%$ under the same conditions and the number of cycles increased from 97 to 166 [3]. The Zn/Ce RFB offers extremely high cell potential; however, the primary challenges it faces are low energy efficiency, low current density, carbon corrosion when using traditional carbon-based electrodes, and high electrode cost when using a platinized electrode. 
$\mathrm{Na}$ et al. developed a cerium/lead flow battery using methanesulfonic acid as the supporting electrolyte and achieved an energy efficiency of $86 \%$ over 800 cycles with a coulombic efficiency of $92 \%$ at a current density of $5 \mathrm{~mA} \mathrm{~cm}^{-2}$ [65]. The cerium-lead RFB utilizes the $\mathrm{Pb} / \mathrm{Pb}^{2+}$ redox couple in the anolyte and the $\mathrm{Ce}^{3+} / \mathrm{Ce}^{4+}$ redox couple in the catholyte. This chemistry offers high redox potential from $\mathrm{Ce}$, which increases power density, and an anolyte that suppresses hydrogen evolution. This is a hybrid RFB as the anolyte undergoes a plating reaction during charge.

Chen et al. demonstrated another hybrid RFB using an aqueous ionic liquid electrolyte, 1-butyl-3-methylimidazolium chloride $(\mathrm{BMImCl})-\mathrm{H}_{2} \mathrm{O}$, with $\mathrm{Zn}$ and cerium redox couples [14]. The zinc-cerium RFB uses the $\mathrm{Zn} / \mathrm{Zn}^{2+}$ redox couple in the anolyte and the $\mathrm{Ce}^{3+} / \mathrm{Ce}^{4+}$ redox couple in the catholyte. In this system, $\mathrm{BMImCl}$ offers high stability against hydrolysis, allowing an expanded electrochemical window of $3 \mathrm{~V}$. In addition, the chlorine ion from $\mathrm{BMImCl}$ can act as the charge carrier with the use of an AEM. Arenas et al. tested different platinum/titanium electrode structures (plate, micromesh, and felt) for use in a zinc/cerium hybrid flow battery [4]. They found that platinized-titanium micromesh is a more effective electrode material and titanium felt with high platinum coverage has the best volumetric mass transport characteristics. Testing was performed at low current densities, 0.025 to $0.075 \mathrm{~mA} \mathrm{~cm}^{-2}$, to reduce the impact of ohmic losses due to the AEM [14].

\subsection{Rationale}

To advance the agenda of RFB technology, it is necessary to develop a low-cost redox chemistry that can compare to the performance metrics of the all-vanadium RFB 
while avoiding the poor performance and longevity of plating reactions. Based on current research, which heavily utilizes traditional carbon-based electrodes, it is clear a new electrode material is needed. Several low-cost redox couples have been explored; however, each suffers from two primary downfalls. One is an insufficient potential, such as the $\mathrm{Fe}^{2+} / \mathrm{Fe}^{3+}$ redox couple with a standard electrode potential of $0.77 \mathrm{~V}$. A low operating potential results in a low power density. This requires more and larger electrochemical cells to meet power requirements, significantly increasing the systems capital cost. The second primary downfall is the existence of carbon corrosion and oxygen evolution at high potentials. The study by Leung et al. makes clear that traditional carbon-based electrodes cannot maintain their integrity in the environment imposed by a $\mathrm{Ce}^{3+} / \mathrm{Ce}^{4+}$ redox couple. Additionally, the low energy efficiency shows evidence of significant efficiency loss due to gas evolution at the cathode electrode.

BDD can answer both challenges presented above through its significantly larger solvent window and extreme resistance to corrosion. With these traits, redox couples such as $\mathrm{Ce}^{3+} / \mathrm{Ce}^{4+}$ and $\mathrm{Mn}^{2+} / \mathrm{Mn}^{3+}$ can be utilized without corrosion and with less gas evolution, increasing the service life of the electrode and the energy efficiency of the system. The aim of Chapter 5 is the demonstration of BDD's viability with high potential, low-cost redox couples in a RFB.

\subsection{Fabrication and characterization of BDD}

To test BDD as an electrode in electrochemical systems, BDD must be obtained. Currently, there are several manufacturers of BDD samples that can be readily purchased and tested. However, the user has very little control over the key characteristics of these 
samples. The ability to fabricate these samples in-house offers extensive benefits. Fabrication allows for control of crystal size, crystal purity, graphitic content, boron doping levels, thickness, and surface functionalization. The quantification and verification of each characteristic allows for intelligent reasoning to be applied to the optimization of BDD for use as an electrode in electrochemical systems. Two reactors are used for the grow of BDD and are referred to as the small Seki and the big Seki; both purchased from Seki Diamond Systems. The big Seki is capable of a higher power and has a heated stage.

Microwave-assisted plasma (MAP) chemical vapor deposition (CVD) was used to grow thin films of BDD onto various substrates. Hydrogen was used as the carrier gas, which also serves the purpose of preferentially etching $\mathrm{sp}$ and $\mathrm{sp}^{2}$ carbon phases, allowing for a more pure crystal phase [61]. Hydrogen plasma also etches $\mathrm{sp}^{3}$ carbon (diamond); however, the etch rate of $\mathrm{sp}^{3}$ is slower than $\mathrm{sp}$ and $\mathrm{sp}^{2}$. As a result, hydrogen supports the growth of $\mathrm{sp}^{3}$ carbon. Methane was used as the carbon source for diamond growth. Operating in a methane starved environment results in larger crystal sizes and less NDC content. Boron powder or diborane gas $\left(\mathrm{B}_{2} \mathrm{H}_{6}\right)$ provides the doping source and is implemented into the diamond crystal structure as it grows producing conductive, P-type, BDD. Figure 26 shows an SEM image of BDD, grown with boron powder as the dopant source, on a conductive silicon substrate. 


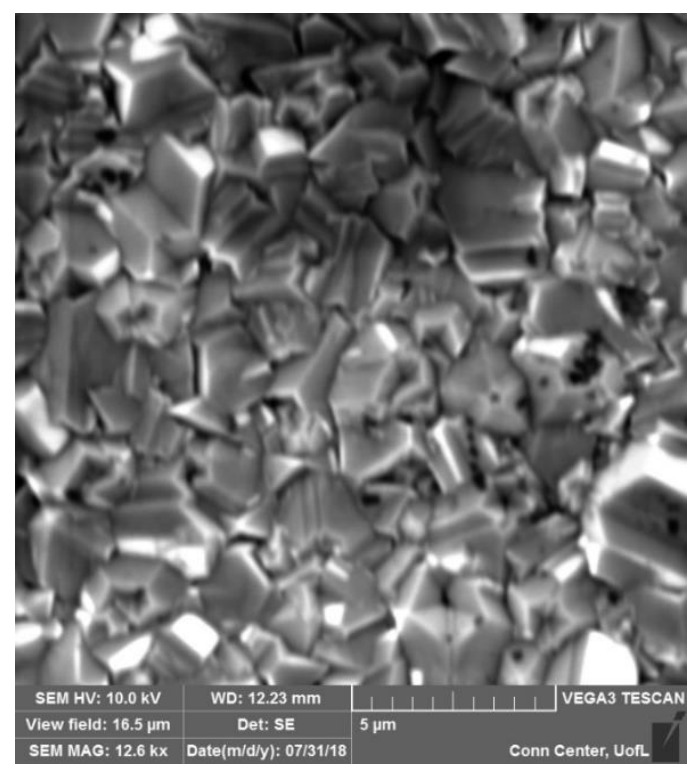

Figure 26. SEM image of a boron doped diamond surface showing continuous crystal growth across a silicon wafer surface.

The crystallinity of the diamond is clear from this image, as is the continuous diamond structure, absent gaps or pinholes. The drawback of using boron powder is the inability to control the boron doping content and difficulty in keeping the substrate surface clear of debris prior to commencement of the plasma.

BDD electrode were also fabricated using tungsten and molybdenum as substrates to determine the difficulty of growing on metal substrates and the resulting advantages and disadvantages. Growth on a metal substrate, which has a higher conductivity than p-type silicon, resulted in a reduction of the peak separation observed by $\mathrm{CV}$; this data is presented in section 5.4. A BDD thin film on a tungsten substrate at different magnifications is show in Figure 27. These images show good coverage of microcrystalline BDD. After repeated use, delamination of the BDD film was observed on the molybdenum and tungsten substrates. This is a result of mechanical strain at the interface of the substrate and BDD 
thin film, which is due to the different thermal expansion coefficient of the contacting materials. During fabrication, temperatures in the plasma have been observed to range from 600 to $1000{ }^{\circ} \mathrm{C}$.

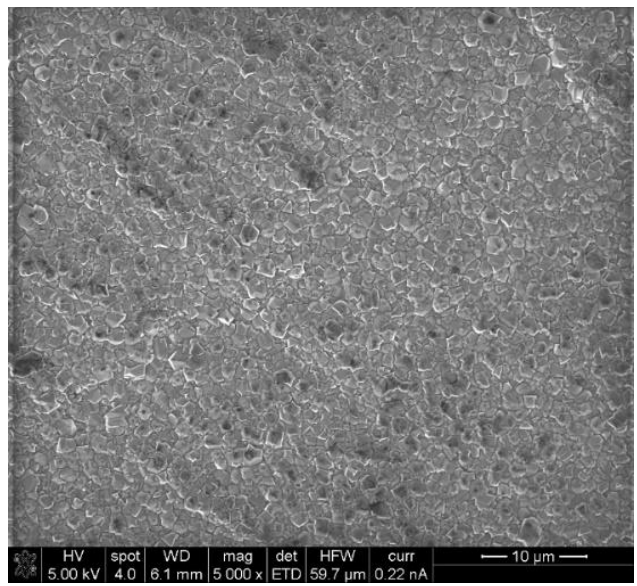

(a)

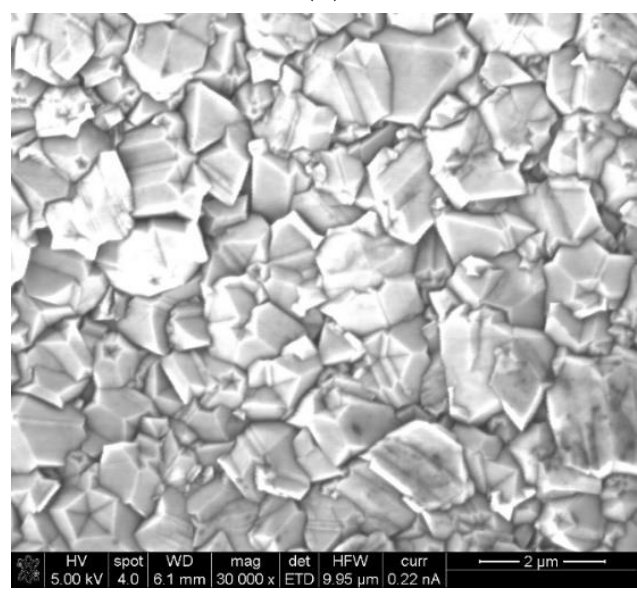

(c)

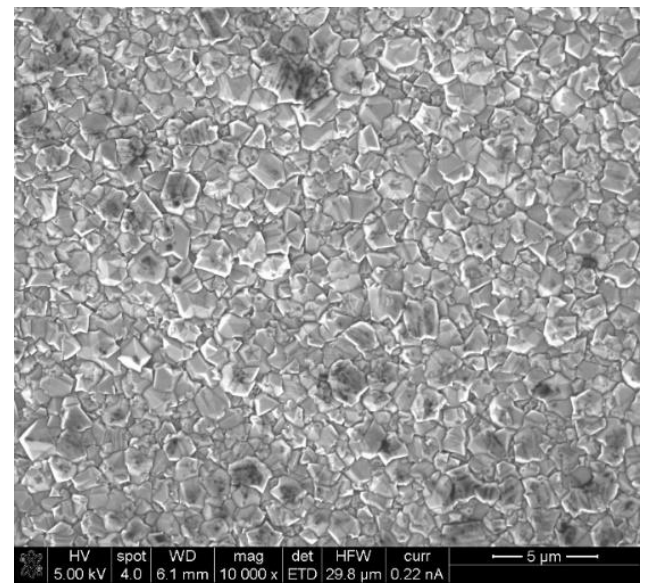

(b)

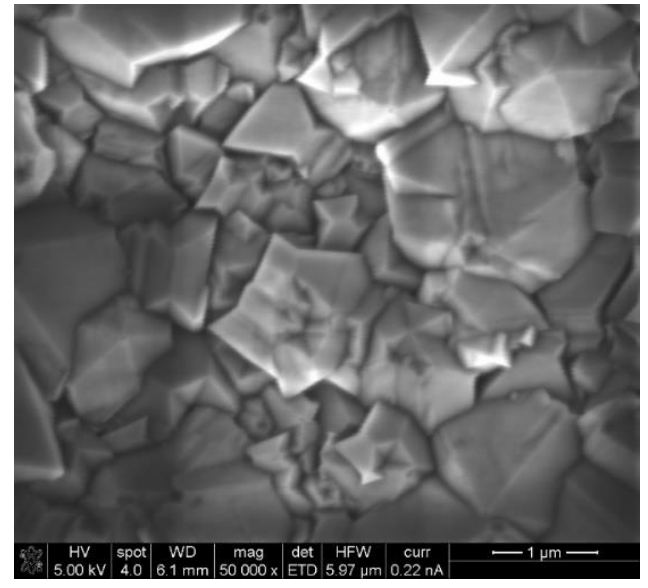

(d)

Figure 27. SEM images of a BDD thin film on a tungsten substrate at (a) 5,000x, (b) 10,000x, (c) 30,000x, and (d) 50,000x magnification.

Fabrication of BDD in the small Seki was done using a microwave power of 1.2 $\mathrm{kW}$ with a chamber pressure of 40 torr. The growth rate of this configuration was found to be $\sim 800 \mathrm{~nm}$ in 40 hours or $20 \mathrm{~nm}_{\text {hour }}{ }^{-1}$, shown in Figure 28 . This is relatively slow 
compared to other growth recipes and conditions. When the BDD films are too thin, pinholes may be present. Pinholes are locations were diamond has not completely grown together, leaving the substrate exposed. Pinholes can become a catalyst to delamination, which is disastrous to the BDD electrode. Additionally, it is not desirable to have the substrate exposed to the electrolyte solution during testing as it can corrode or result in undesired side reaction. To combat the slow growth rate of BDD in the small Seki, alleviate concerns of polluting the big Seki with boron, and remove any possibility of pinholes; nitrogen incorporated growth of diamond was performed. Introducing nitrogen into diamond growth has been shown to substantially increase the growth rate [5]. Nitrogen incorporated diamond on a silicon substrate is shown in Figure 29(a). Growth with nitrogen results in a different morphology, dependent on the amount of nitrogen present. Increased nitrogen content results in the involvement of secondary nucleation sights and an increase in porosity [94]. The growth rate of nitrogen incorporated diamond in the big Seki reactor with a microwave power of $1.6 \mathrm{~kW}$ and chamber pressure of 60 torr is $\sim 24 \mu \mathrm{m}$ in 60 hours of growth or $400 \mathrm{~nm}$ hour $^{-1}$, shown in Figure 29(b). Additionally, the big Seki reactor has a heated stage which was set to $850{ }^{\circ} \mathrm{C}$ and results in a much higher growth temperature. 


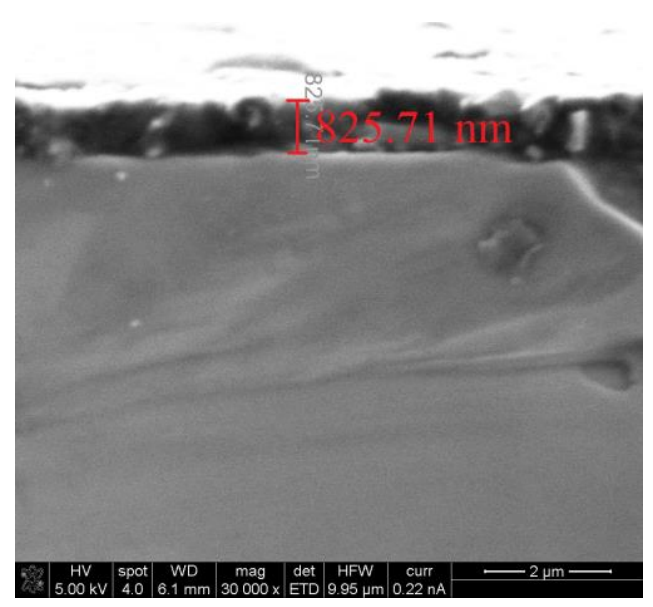

Figure 28. SEM images showing the cross section of a BDD film on silicon substrate grown in the small Seki reactor with a microwave power of $1.2 \mathrm{~kW}$ and chamber pressure of 40 torr.

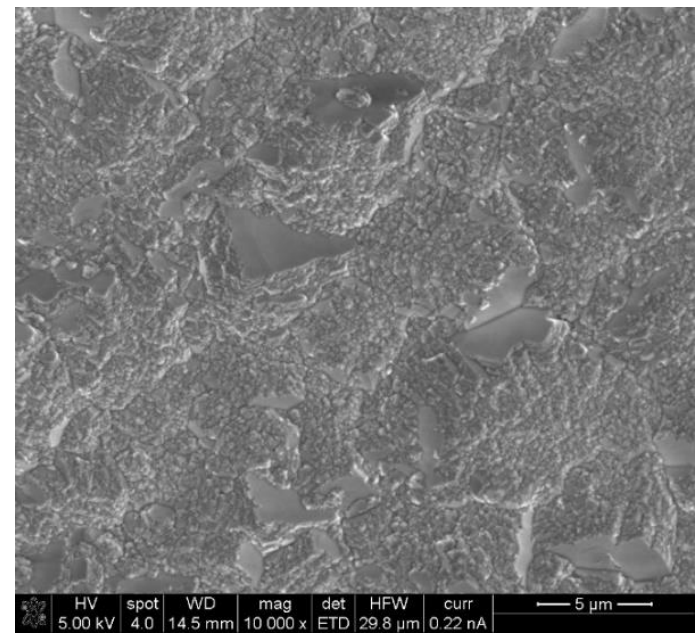

(a)

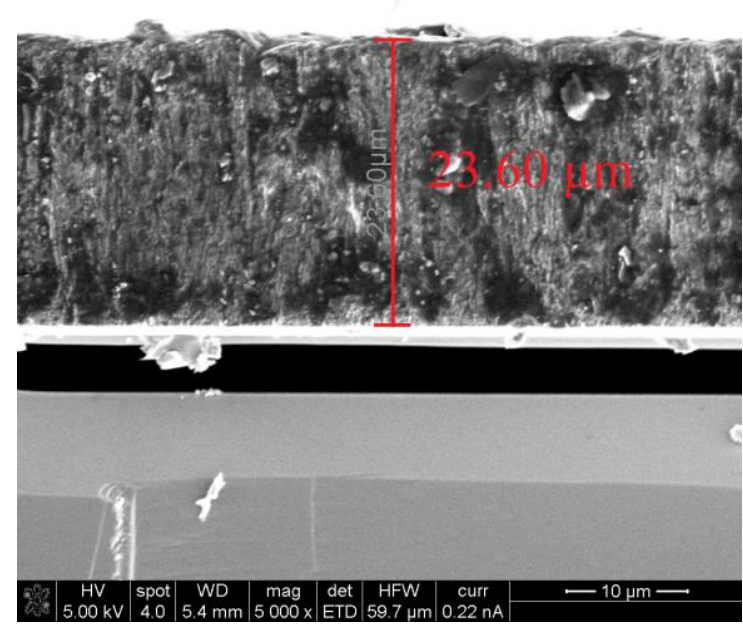

(b)

Figure 29. SEM images showing the (a) surface and (b) cross section of nitrogen incorporated diamond on a silicon substrate grown in the big Seki reactor with a microwave power of $1.6 \mathrm{~kW}$, chamber pressure of 60 torr, and stage temperature of $850{ }^{\circ} \mathrm{C}$. 
To vary the boron doping concentration, the amount of boron entering the reaction chamber must be controllable. This is accomplished by utilizing diborane gas as opposed to boron powder. The flow rate of diborane gas can be controlled, thus controlling the amount of boron entering the reaction chamber. This will allow for variation of the boron content and optimization of the BDD electrode to have the highest conductivity possible while keeping NDC content to a minimum. Figure 30 shows BDD grown on a silicon substrate using diborane gas as opposed to boron powder.

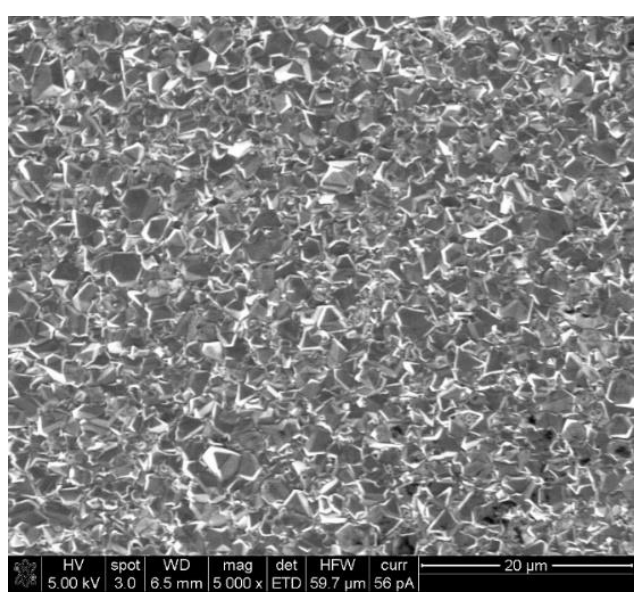

(a)

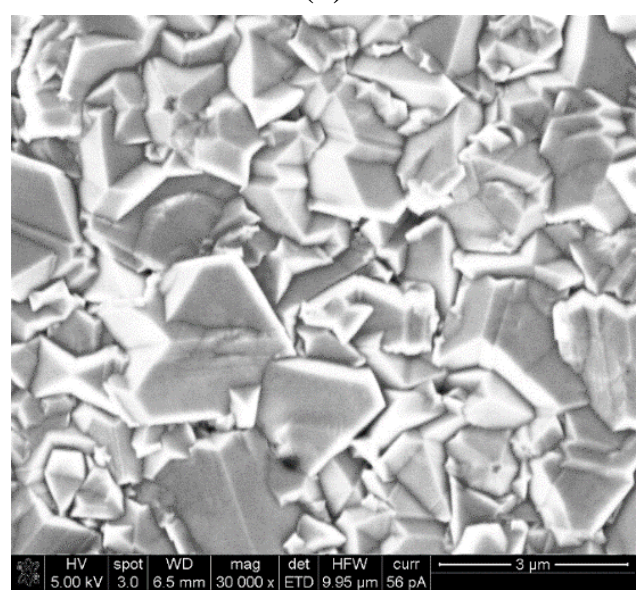

(c)

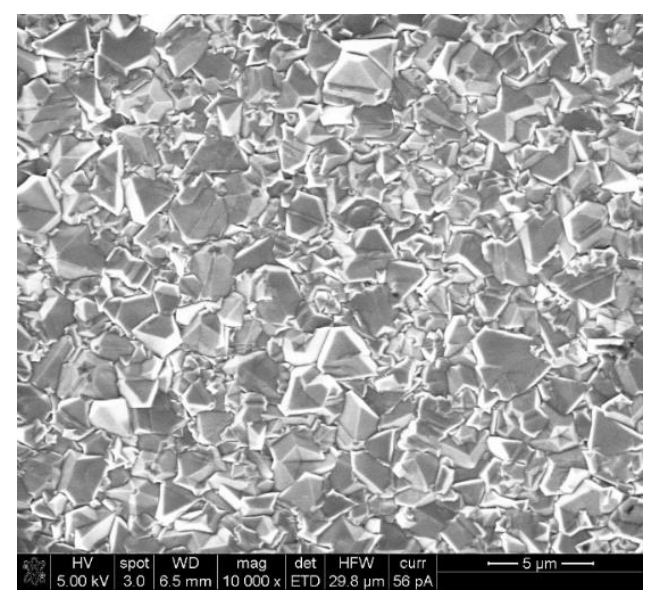

(b)

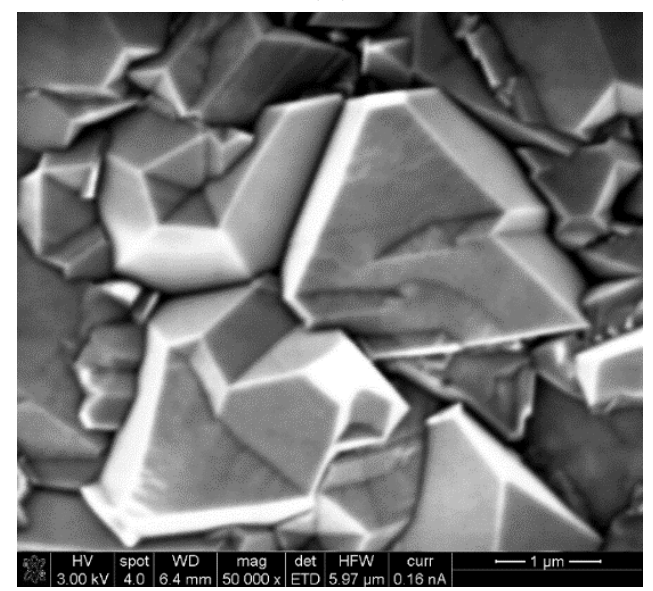

(d)

Figure 30. BDD grown on a silicon substrate using diborane gas at (a) 5,000x, (b)

$$
\text { 10,000x, (c) 30,000x, and (d) 50,000x. }
$$


Additional characterization of BDD was done using Raman spectroscopy. Raman was preformed of a BDD thin film on a tungsten substrate, shown in Figure 31. This figure, showing Raman scans at multiple positions, exhibits a strong peak at $1332 \mathrm{~cm}^{-1}$, which corresponds to the $\mathrm{sp}^{3}$ peak of diamond. There are no other peaks or shoulders evident in these scans, indicating a pristine diamond film with minimal NDC content.

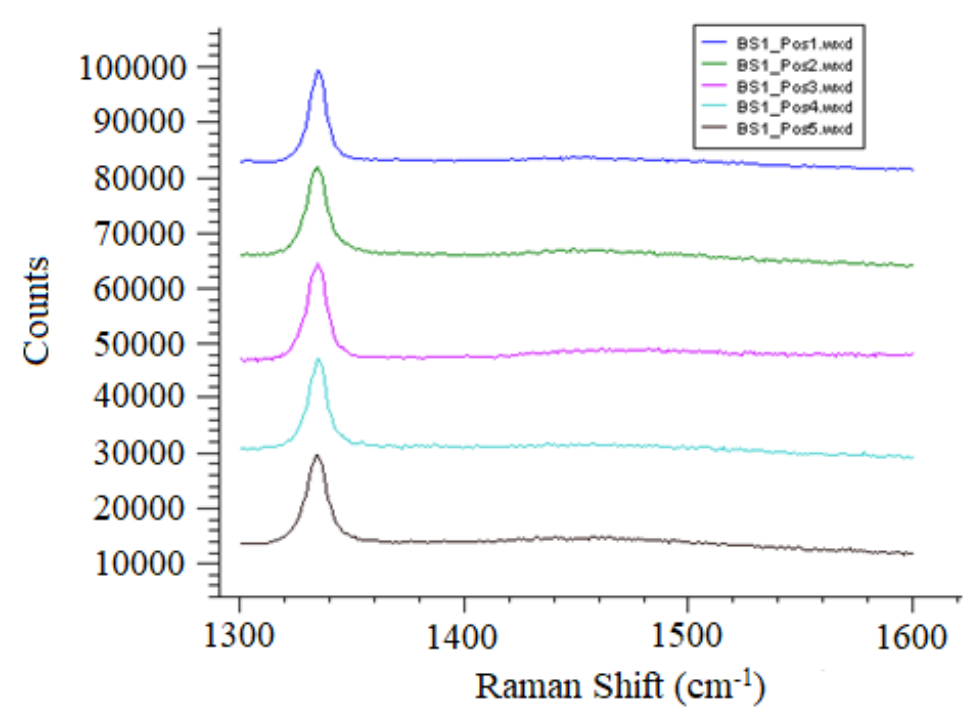

Figure 31. Raman of a BDD thin film on a tungsten substrate showing the diamond, $\mathrm{sp}^{3}$ peak, at random positions on the diamond surface.

Advancing this objective requires repeatability in the fabrication of BDD while using diborane gas as the boron source. Previous fabrication methods employed boron powder as the boron source, which does not allow for control of the boron content in the BDD samples. Mass flow controllers with a sufficient resolution to control the diborane gas flow were recently obtained. Repeatability will be confirmed through SEM, Raman, and electrochemical testing of the solvent window in a sulfuric acid solution. Once 
repeatable fabrication of the BDD electrodes is verified, the modification of the BDD recipe can move forward.

To explore the effects of BDD modification, different samples must be fabricated and tested under the same conditions. Different modifications include varying growth conditions and utilizing nitrogen incorporated diamond as a substrate for BDD. Growth conditions that can be varied include power, temperature, pressure, time, and gas flow rates. These conditions control crystal size, crystal purity, graphitic content, boron doping levels (conductivity), thickness, and surface functionalization. As the primary concern of this objective is the effect of modification on BDD's ability as a RFB electrode, the primary means of testing should be electrochemical; however, non-electrochemical characterization can offer a fast and easy comparison. Electrochemical testing will include $\mathrm{CV}$ to show the solvent window and $\mathrm{CV}$ with an active species in the electrolyte to see if the reversibility and/or reaction kinetics have been affected. SEM will allow comparison of crystal sizes and morphology. Raman will show qualitative differences in NDC content, crystallinity of the diamond itself, and boron doping content. Secondary ion mass spectrometry (SIMS) will be performed on select samples to determine the boron content quantitatively. This will give a deeper meaning to results obtained by Raman. Additionally, XPS may be viable to show changing surface functional groups based on different modifications.

To test BDD as an electrode in electrochemical systems, BDD must first be fabricated. Currently, there are several manufacturers of BDD samples that can be readily purchased and tested. However, the user has very little control over the key characteristics of these samples. The ability to fabricate these samples in-house offers extensive benefits. 
Fabrication allows for control of crystal size, crystal purity, graphitic content, boron doping levels, thickness, and surface functionalization. The quantification and verification of each characteristic allows for intelligent reasoning to be applied to the optimization of BDD for use as an electrode in electrochemical systems. Two reactors are used for the growth of BDD and are referred to as the small Seki and the big Seki; both purchased from Seki Diamond Systems. The big Seki is capable of a higher power and has a heated stage.

FTIR spectra is shown in Figure 32. for an as-grown BDD sample. The strong absorption peaks at 1980, 2030, 2160, and $2360 \mathrm{~cm}^{-1}$ are intrinsic diamond peaks and appear in the spectra of all diamonds [27; 28; 44]. The small peak at $2450 \mathrm{~cm}^{-1}$ represents the ground state to first excited state electronic transition of neutral boron acceptors [16]. A peak at $2800 \mathrm{~cm}^{-1}$, indictive of the ground state to second excited state electronic transition of neutral boron acceptors, is not observed due to hydrogen passivation [16]. The peak at 2850 and 2920 represent $\mathrm{sp}^{3}$ bonded $\mathrm{CH}_{3}$ and $\mathrm{sp}^{3}$ bonded $\mathrm{CH}_{2}$, respectively [26]. Nitrogen impurity peaks would be observed at $1130 \mathrm{~cm}^{-1}$, C-form, and $1344 \mathrm{~cm}^{-1}$, Aform [43]. These peaks are not evident in the FTIR spectra presented in Figure 32 indicating little to no nitrogen impurities in the BDD samples used in this work. 


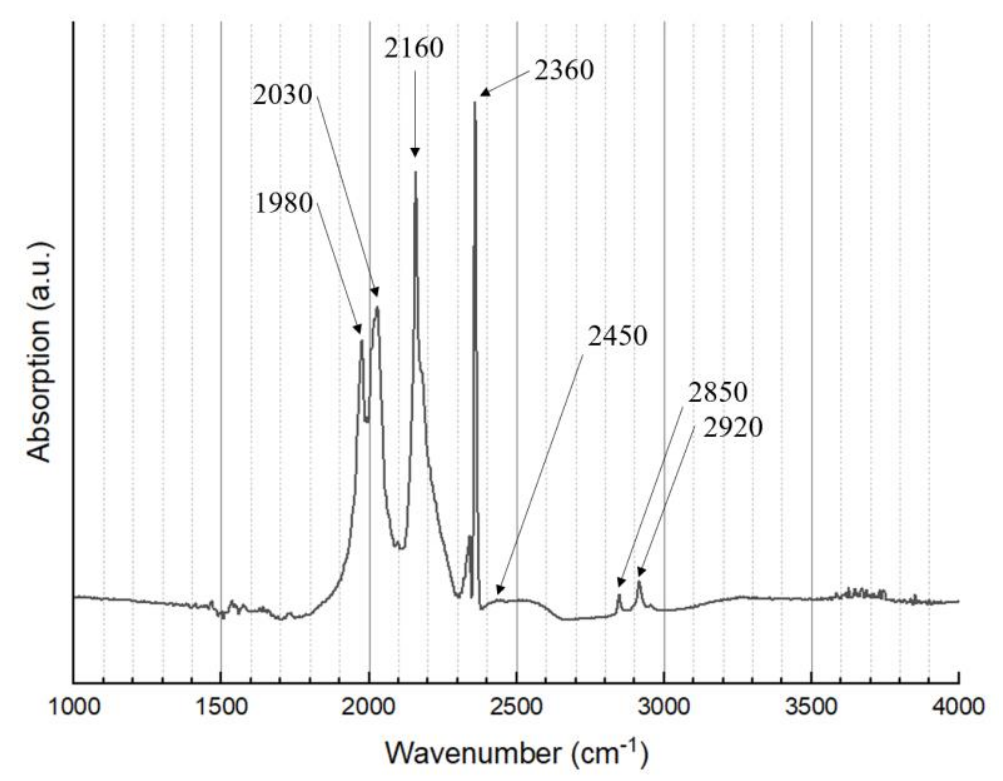

Figure 32. FTIR spectra of as-grown BDD.

\subsection{Performance of the $\mathrm{Mn}^{2+} / \mathrm{Mn}^{3+}$ and $\mathrm{Ce}^{3+} / \mathrm{Ce}^{4+}$ redox couples on BDD}

The manganese redox couple, $\mathrm{Mn}^{2+} / \mathrm{Mn}^{3+}$, has shown promise to replace the allvanadium catholyte in RFBs. The $\mathrm{Mn}^{2+} / \mathrm{Mn}^{3+}$ redox couple has a higher potential then the $\mathrm{V}^{4+} / \mathrm{V}^{5+}$ catholyte with a comparable solubility, which allows for better power and energy density. manganese has been shown to be highly reversible, with a very small peak-to-peak separation in CV curves and a peak height ratio near one [23]. The biggest benefit to manganese is its cost compared to vanadium. Replacing vanadium with manganese will substantially lower the capital cost of RFB systems and make them appealing for grid energy storage from a financial perspective.

Previously, the $\mathrm{Mn}^{2+} / \mathrm{Mn}^{3+}$ redox couple was tested for use in a RFB using graphite felt electrodes [23]. The results of testing were positive in terms of reversibility and energy efficiency; however, at the potentials required for the $\mathrm{Mn}^{2+} / \mathrm{Mn}^{3+}$ redox couple to operate are known to corrode graphite. The corrosion of graphite in a RFB will decrease longevity 
and can lead to catastrophic failure through leaking of the highly corrosive electrolyte. Figure 33 shows $\mathrm{CV}$ curves for the $\mathrm{Mn}^{2+} / \mathrm{Mn}^{3+}$ redox couple on BDD using a silicon and substrate. These CV curves are compared to CV curves using platinum foil as a working electrode. This comparison was done due to the catalytic nature of platinum; reactions typically proceed very fast on its surface making it the ideal case. From Figure 33, it is seen that the $\mathrm{Mn}^{2+} / \mathrm{Mn}^{3+}$ redox couple on BDD compares very well with platinum. The oxidation peak of the BDD electrode is within $10 \mathrm{mV}$ of the platinum electrode. The peak-to-peak separation of $\mathrm{Mn}^{2+} / \mathrm{Mn}^{3+}$ on platinum was found to be $144 \mathrm{mV}$ while the peak-to-peak separation of $\mathrm{Mn}^{2+} / \mathrm{Mn}^{3+}$ on BDD was found to be $180 \mathrm{mV}$. The peak height ratio of $\mathrm{Mn}^{2+} / \mathrm{Mn}^{3+}$ on platinum was calculated to be 2.62 with the peak height ratio on BDD calculated as 1.74. A peak height ratio close to one indicates excellent reversibility and the low peak-to-peak separation indicates low overpotentials required for reactions to proceed. Low overpotentials for reduction and oxidation leads to high energy efficiencies.

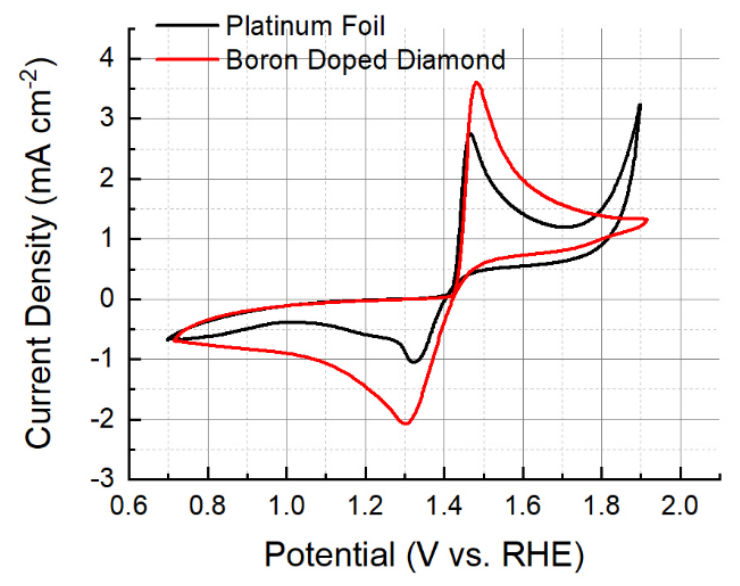

Figure 33. CV of a BDD electrode at a scan rate of $3 \mathrm{mV} \mathrm{s}^{-1}$ in an electrolyte solution consisting of $0.1 \mathrm{M} \mathrm{MnSO}_{4}$ and $0.5 \mathrm{M} \mathrm{H}_{2} \mathrm{SO}_{4}$. 
Figure 34 shows $\mathrm{CV}$ results for the $1^{\text {st }}$ and $180^{\text {th }}$ scan of the $\mathrm{Mn}^{2+} / \mathrm{Mn}^{3+}$ redox couple on a BDD electrode. The oxidation peak height remains nearly the same as the first scan with the peak potential moving in slightly. The reduction peak height is slightly reduced; however, the peak height ratio in the $180^{\text {th }}$ scan is closer to one as compared to the first scan; 1.29 and 1.70, respectively. This indicates good cyclability with no degradation in performance of the $\mathrm{Mn}^{2+} / \mathrm{Mn}^{3+}$ redox couple while operating on BDD.

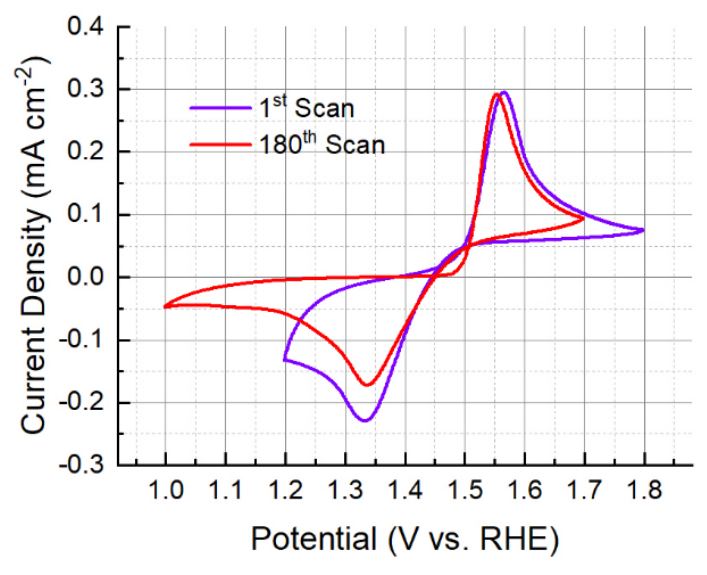

Figure 34. $\mathrm{CV}$ scans of $10 \mathrm{mM} \mathrm{Mn}\left(\mathrm{NO}_{3}\right)_{2}$ in $1 \mathrm{M} \mathrm{HNO}_{3}$ at a scan rate of $1 \mathrm{mV} \mathrm{s}^{-1}$ before and after 180 cycles.

Replacing the catholyte of the all-vanadium RFB with manganese would substantially change the game for RFBs in industry with regard to capital costs. Still, consideration must be taken into which redox couple will be paired with manganese to form the anolyte. A big advantage to the all-vanadium flow battery is low cross over of the active species between the anolyte and catholyte. Because the same chemical species is present, there are no substantial concentration gradients forcing crossover. Some crossover still occurs due to migration; however, it is not severely detrimental to the lifetime of the RFB system and is relatively easily reversed by mixing and redistribution of the anolyte 
and catholyte. A manganese catholyte and vanadium anolyte system is possible, as has been shown in literature but, it may not be practical due to issues arising from crossover concerns [41]. So, it would be beneficial to find an anolyte redox couple, such as $\mathrm{Ti}$, that can operate with manganese as a mixed electrolyte.

The use of the $\mathrm{Ce}^{3+} / \mathrm{Ce}^{4+}$ redox couple in RFBs has been more extensively studied compared to manganese $[55 ; 64 ; 68 ; 98 ; 105]$. The $\mathrm{Ce}^{3+} / \mathrm{Ce}^{4+}$ redox couple is cost-effective relative to $\mathrm{V}$, has a high positive standard potential

$$
\mathrm{Ce}^{4+}+e^{-} \leftrightarrow \mathrm{Ce}^{3+}
$$

and does not undergo a disproportionation reaction like Mn. However, it does suffer from low solubility which restricts the electrolyte energy density [69]. Figure 35 shows CV scans

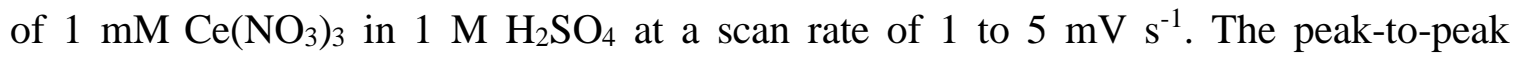
separation was $254.8 \mathrm{mV}$ at a scan rate of $1 \mathrm{mV} \mathrm{s}^{-1}$ with a formal potential of $1.406 \mathrm{~V}$ vs. RHE. The formal potential for the $\mathrm{Ce}^{3+} / \mathrm{Ce}^{4+}$ redox couple agrees well with the standard thermodynamic potential in $\mathrm{H}_{2} \mathrm{SO}_{4}$ [10]. The peak-to-peak separations achieved on BDD are consistent with those reported in literature. $\mathrm{Na}$ et al. examined the $\mathrm{Ce}^{3+} / \mathrm{Ce}^{4+}$ redox couple with $50 \mathrm{mM} \mathrm{Ce}\left(\mathrm{CH}_{3} \mathrm{SO}_{3}\right)_{3}$ in $1 \mathrm{M} \mathrm{CH}_{3} \mathrm{SO}_{3} \mathrm{H}$ using surface-functionalized graphite felt [64]. The peak-to-peak separation obtained by $\mathrm{Na}$ et al. was $\sim 222 \mathrm{mV}$, at a scan rate of $3 \mathrm{mV} \mathrm{s}^{-1}$, for each graphite felt electrode tested [64]. In a study by Nikiforidix et al., the peak-to-peak separation for the $\mathrm{Ce}^{3+} / \mathrm{Ce}^{4+}$ redox couple was found to be $290 \mathrm{mV}$ at room temperature, on a Pt-Ti mesh, in an electrolyte containing $800 \mathrm{mM}$ Ce in $4.4 \mathrm{M} \mathrm{CH}_{3} \mathrm{SO}_{3} \mathrm{H}$ at a scan rate of $40 \mathrm{mV} \mathrm{s}^{-1}$ [68]. 


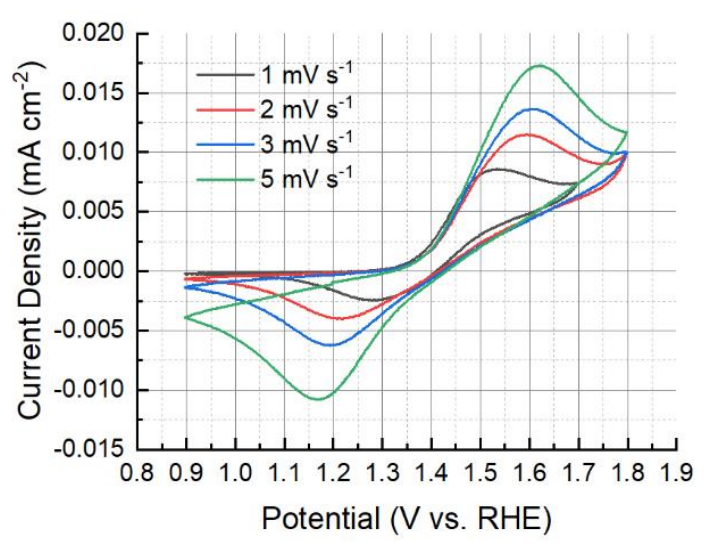

Figure 35. CV scans of BDD working electrodes in $1 \mathrm{mM} \mathrm{Ce}\left(\mathrm{NO}_{3}\right)_{3}$ in $1 \mathrm{M} \mathrm{H}_{2} \mathrm{SO}_{4}$ at scan rates from 1 to $5 \mathrm{mV} \mathrm{s}^{-1}$.

Figure 36 shows $\mathrm{CV}$ scans of $50 \mathrm{mM} \mathrm{Ce} 2\left(\mathrm{CO}_{3}\right)_{3}$ and $50 \mathrm{mM} \mathrm{Ce}\left(\mathrm{SO}_{4}\right)_{2}$ in $1 \mathrm{M}$ $\mathrm{H}_{2} \mathrm{SO}_{4}$, to achieve a 50/50 solution of $\mathrm{Ce}^{3+}$ and $\mathrm{Ce}^{4+}$ ions, enable comparison with a published study by Wang et al., and demonstrate operation with higher active species concentration [98]. The peak-to-peak separation was $350.7 \mathrm{mV}$ at a scan rate of $1 \mathrm{mV} \mathrm{s}^{-1}$ with a formal potential of $1.417 \mathrm{~V}$ vs. RHE. The peak-to-peak separation is consistent with published data. Wang et al. reported a peak-to-peak separation of $476 \mathrm{mV}$ on glassy carbon at a scan rate of $20 \mathrm{mV} \mathrm{s}^{-1}$ in the same electrolyte composition [98]. CV of an electrolyte consisting of $1 \mathrm{mM} \mathrm{Ce}\left(\mathrm{NO}_{3}\right)_{3}$ and $1 \mathrm{M} \mathrm{HNO}_{3}$ on a BDD electrode is shown in Figure 37. In $\mathrm{HNO}_{3}$, the formal potential of the $\mathrm{Ce}^{3+} / \mathrm{Ce}^{4+}$ redox couple shifts to $1.67 \mathrm{~V}$ vs. SHE. The formal potential shift is more than $200 \mathrm{mV}$ compared to cerium in $\mathrm{H}_{2} \mathrm{SO}_{4}$. That represents a large increase in the possible energy and power densities; however, there is a problem of solubility when using $\mathrm{HNO}_{3}$ that must be overcome to take advantage of the larger potential. 


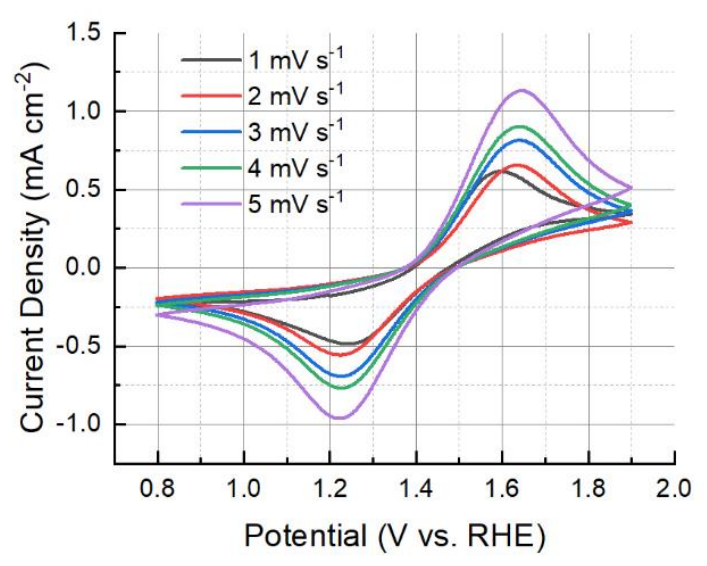

Figure 36. $\mathrm{CVs}$ of $\mathrm{BDD}$ working electrodes in a $50 \mathrm{mM} \mathrm{Ce}_{2}\left(\mathrm{CO}_{3}\right) 3,50 \mathrm{mM} \mathrm{Ce}\left(\mathrm{SO}_{4}\right)_{2}$, and $1 \mathrm{M} \mathrm{H}_{2} \mathrm{SO}_{4}$ electrolyte.

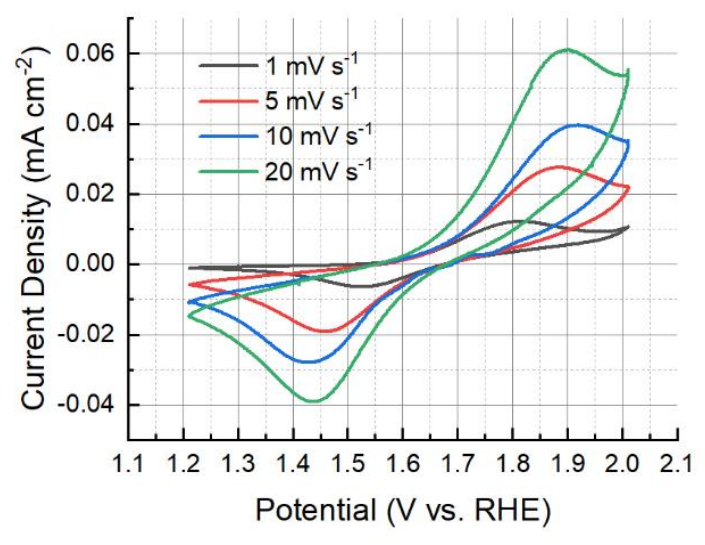

Figure 37. CVs of a BDD working electrode in a $1 \mathrm{mM} \mathrm{Ce}\left(\mathrm{NO}_{3}\right)_{3}$ and $1 \mathrm{M} \mathrm{HNO}_{3}$ electrolyte.

The manganese redox couple, $\mathrm{Mn}^{2+} / \mathrm{Mn}^{3+}$, has shown promise to replace the allvanadium catholyte in RFBs. The manganese redox couple has a higher potential then the vanadium catholyte, which allows for better power density. The solubility of manganese is comparable to vanadium, which means energy density will not suffer. Manganese has been shown to be highly reversible, with a very small peak to peak difference in CV curves and 
a peak height ratio near one [23]. The biggest benefit to manganese is its cost compared to vanadium. Replacing vanadium with manganese will substantially lower the capital cost of RFB systems and make them appealing for grid scale energy storage from a financial perspective.

When beginning this study, it was unknown which, if any, redox couples would be successful on a BDD electrode. Prior to work on BDD electrodes, the manganese redox couple was explored on graphite felt due to the benefits accessible using manganese. So, manganese was one of the first redox couples tested on a BDD electrode and, coincidently, it has been the most successful. Figure 38 shows CV curves for the manganese redox couple on BDD using a silicon and tungsten substrate. These CV curves are compared to CV curves using platinum foil as a working electrode. This comparison was done due to the catalytic nature of platinum; reactions typically proceed very fast on its surface. From Figure 38, it is seen that the manganese redox couple on BDD compares very well with platinum. The oxidation peak of the BDD electrode, on a tungsten substrate, is within $10 \mathrm{mV}$ of the platinum electrode. The sharp peaks and small peak to peak potential difference indicate a reversible reaction. 


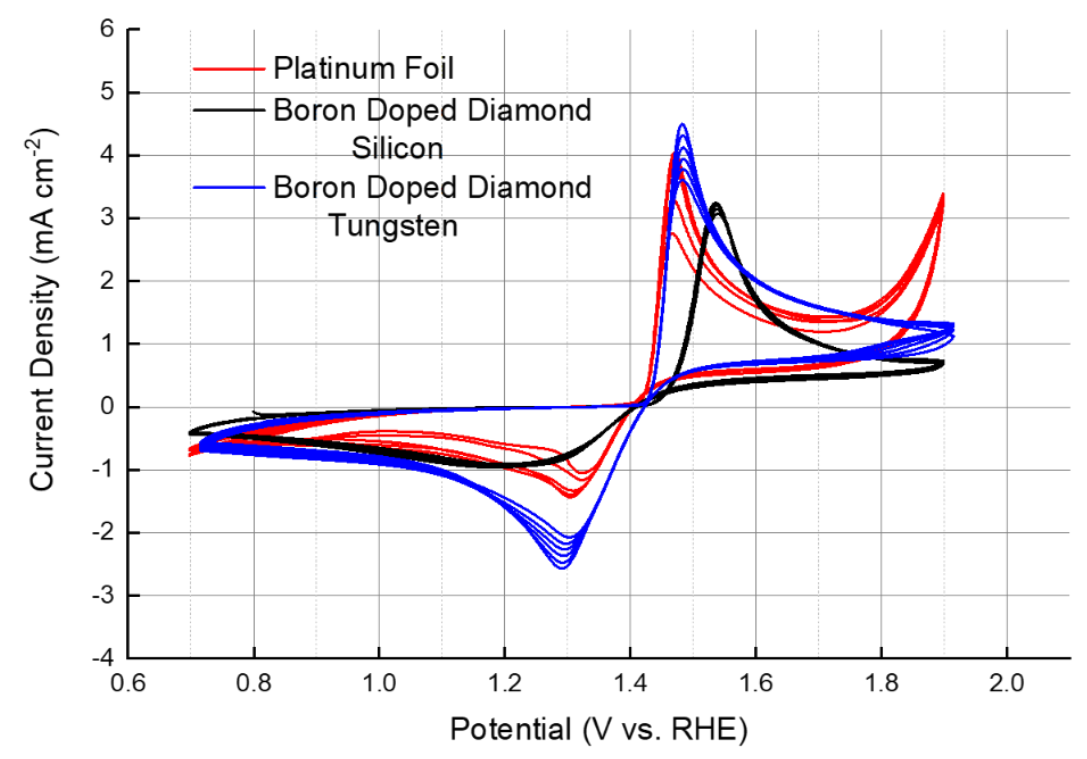

Figure 38. CV of a BDD electrode at a scan rate of $3 \mathrm{mV} \mathrm{s}^{-1}$ in an electrolyte solution consisting of $0.1 \mathrm{M}$ manganese and $0.5 \mathrm{M}$ sulfuric acid. Two BDD substrates, silicon and tungsten, are compared along with platinum foil.

Replacing the catholyte of the all-vanadium RFB with manganese would substantially change the game for RFBs in industry with regard to capital costs. Still, consideration must be taken into which redox couple will be paired with manganese to form the anolyte. A big advantage to the all-vanadium flow battery is low cross over of the active species between the anolyte and catholyte. Because the same chemical species is present, there are no substantial concentration gradients forcing crossover. Some crossover still occurs due to migration; however, it is not severely detrimental to the lifetime of the RFB system and is relatively easily reversed by mixing and redistribution of the anolyte and catholyte. A manganese catholyte and vanadium anolyte system is possible, as has been shown in literature but, it may not be practical due to issues arising from crossover 
concerns [41]. So, it would be beneficial to find an anolyte redox couple that better matches with manganese as the catholyte.

Although some success has been seen in using the manganese redox couple on $\mathrm{BDD}$, this reaction must be more thoroughly examined. This includes exploration into why it was successful and ways to improve on its function. A combination of electrochemical testing techniques, including the rotating disk electrode, $\mathrm{CV}$, and EIS, will be utilized to gain insight on the reaction mechanism of the manganese redox couple on the BDD electrode. The amount of $\mathrm{MnO}_{2}$ precipitate that occurs on the BDD electrode should be quantified and compared to the amount that occurs on a graphite electrode. This will determine if there is any benefit to the precipitation reaction from using BDD as an electrode. Also, the precipitate will be dried and tested using XRD to determine its phase and compare that to the published literature. The anolyte redox couple has not been chosen; however, it will undergo testing similar to the catholyte redox couple presented above. It is desired to understand the reaction mechanism on the BDD electrode surface to facilitate optimization of the RFB. Additional testing of the anolyte will include determination of the solubility in varying concentrations of acid and optimization of the active species and supporting electrolyte ratios.

\subsection{Durability of BDD as an electrode in RFBs}

Graphite based electrodes are commonly used in aqueous electrochemical systems, especially RFBs, as they work great in low potential settings. They are conductive, inert, and resist fouling in most corrosive environments. However, when potentials increase beyond a point, fouling becomes very apparent as the graphite surface is oxidized. This 
causes graphite to corrode resulting in severe pitting and eventual degradation of the entire surface. Within the potential window of the all-vanadium RFB, graphite is reasonably stable; however, venturing outside of those potentials is detrimental to the lifetime of graphite, which in turn is devastating to the RFBs' use as a grid scale energy storage device. Maintenance costs for replacing electrodes in a RFB are substantial, as the entire RFB would need to cease operation and be disassembled. Also, contamination of the electrolyte would need to be dealt with. Degradation of graphite used as flow fields would result in chemical leaks which is dangerous to human life as well as the surrounding environment. Other electrodes have been tested for use in RFBs but, none have exhibited the abilities of graphite. BDD has been shown to resist fouling in high temperature, corrosive, and high potential environments [61]. Because BDD has previously not been used as an electrode for RFBs, its fouling resistance must be examined in the environments utilized by RFBs.

A BDD electrode on a silicon substrate was used to preform CV in a manganese electrolyte. SEM showed a significant amount of material on the electrode surface, which was verified to be manganese through EDX, shown in Figure 39. Figure 39 ( $a$ and b) were taken at a location with only diamond observable in the SEM. As a result, the main EDX peak corresponds to carbon which makes up $89.84 \%$ by weight of the locations composition. Another $8.17 \%$ by weight consists of oxygen and $1.29 \%$ by weight silicon. The oxygen and silicon are believed to be mainly artifacts of the silicon substrate, which EDX can partially see, underneath the BDD thin film. Figure 39 (c and d) were taken at a location with no observable diamond crystals and a large amount of some other substance. EDX results found this substance to be mainly manganese, with $45.88 \%$ by weight. Carbon and oxygen are also present with $33.59 \%$ and $19.62 \%$ by weight, respectively. The form 
of the manganese present is expected to be $\mathrm{MnO}_{2}$, which correlates well with the increase in oxygen content at this location. The carbon content is believed to be a result of the diamond layer underneath this material deposit.

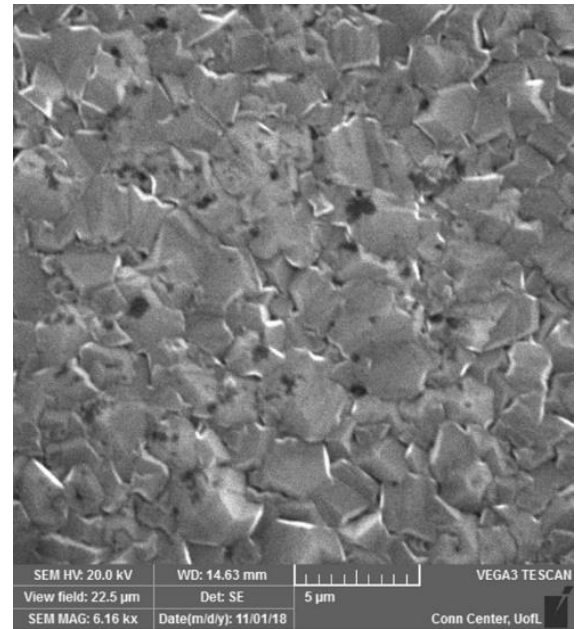

(a)

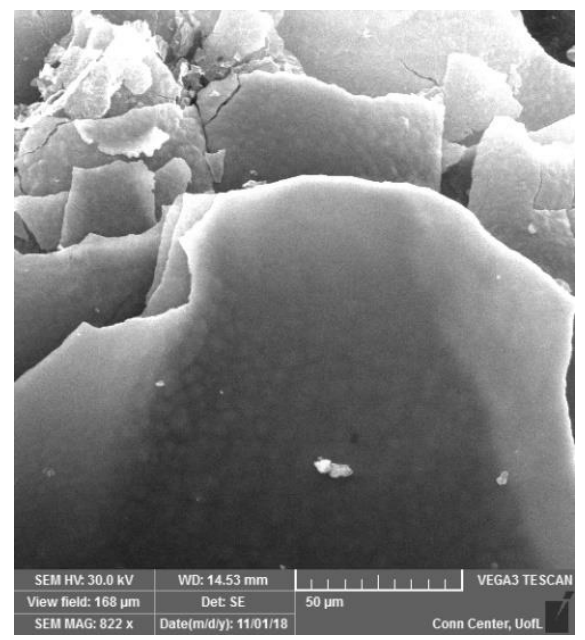

(c)

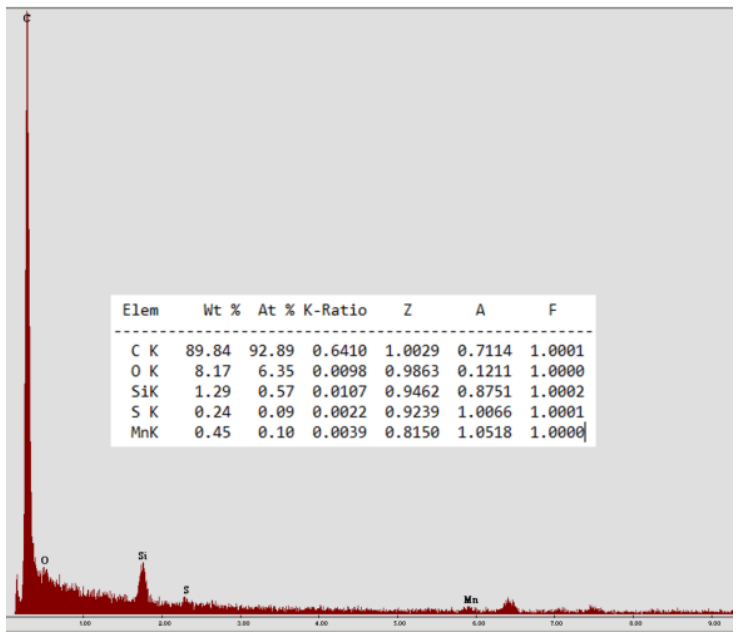

(b)

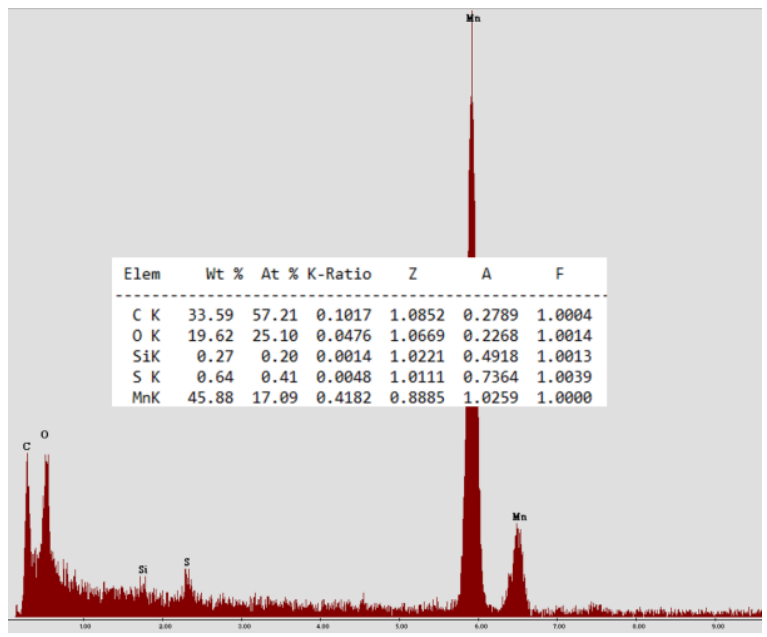

(d)

Figure 39. SEM and EDX of a BDD thin film on a silicon substrate after CV cycling in a manganese electrolyte. ( $a$ and $b$ ) were taken at a location with only diamond observable on the electrode surface. (c and d) were taken at a location with significant deposits of non-diamond material. 
A Raman spectrum, after CV cycling in a manganese electrolyte, was taken of the same electrode shown in Figure 40. The Raman spectrum, shown in Figure 40, was taken at multiple locations on the electrode surface, including locations which might include possible fouling. The Raman spectra exhibit a large sharp peak at the $1332 \mathrm{~cm}^{-1}$, with a small broad peak around $1500 \mathrm{~cm}^{-1}$. The broad peak indicates the possibility of NDC content; however, compared to published data, this sample is mostly pristine BDD [107]. After a thorough cleaning, it is expected that this Raman spectra will improve.

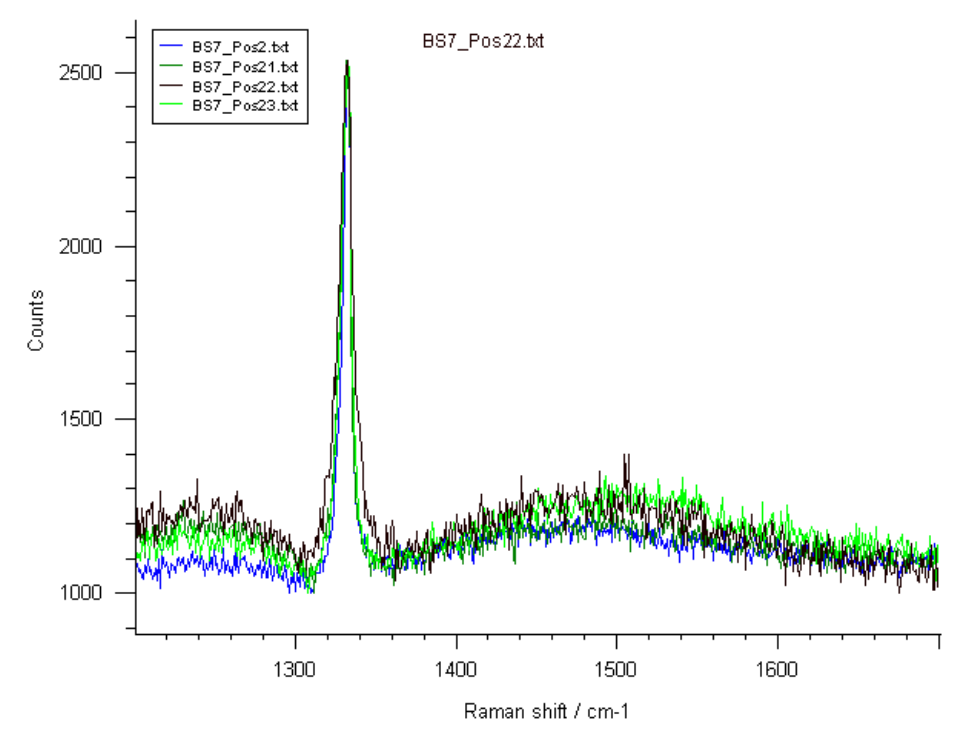

Figure 40. Raman of a BDD thin film on a silicon substrate.

BDD electrodes have been tested using $\mathrm{CV}$ for many cycles from $-2 \mathrm{~V}$ to $3 \mathrm{~V}$ (vs. $\mathrm{Ag} / \mathrm{AgCl}$ ) in $0.5 \mathrm{M}$ sulfuric acid and shown negligible fouling based on little degradation of peak magnitudes and position in CV results, as well as visual inspection of the electrodes. Moreover, the degradation of a graphite electrode has been observed after CV cycling in a manganese electrolyte. The electrolyte solution color turned black and 
after removing the graphite working electrode, an observable amount of material has been removed. The graphite electrode appears to have pitting corrosion.

To demonstrate the fouling of graphite, a before and after test should be performed in the manganese electrolyte. Characterization of morphology changes in the graphite by SEM will be sufficient to demonstrate pitting corrosion. The graphite sample will also be weighed before and after testing. Weight loss of the graphite rod represents material lost to the electrolyte during electrochemical testing. For the BDD, CV will be performed for a number of cycles and the sample will be characterized before and after. Morphology changes can be observed by SEM. Proper cleaning of the BDD surface is required to remove any precipitated materials so that the morphology of the diamond can be obtained. Raman spectra will give insight into the presence of NDC. Care must be taken; however, not to confuse results with those of changing surface functionality. To that end, the BDD electrode should be characterized between large sets of cycles in only sulfuric acid to determine if any stabilization of the electrode occurs. Once stabilized, the above stated methodology should be applied in a manganese electrolyte.

Graphite based electrodes are commonly used in aqueous electrochemical systems, especially RFBs, as they work great in low potential settings. They are conductive, inert, and resist fouling in most corrosive environments. However, when potentials increase beyond a point, carbon corrosion becomes very apparent as the graphite surface is oxidized. This results in severe pitting and eventual degradation of the entire surface. Within the potential window of the all-vanadium RFB, graphite is reasonably stable; however, venturing outside of those potentials is detrimental to the lifetime of graphite, which in turn is devastating to the RFBs' use as a grid scale energy storage device. Maintenance costs 
for replacing electrodes in a RFB are substantial, as the entire RFB would need to cease operation and be disassembled. Also, contamination of the electrolyte by carbon particles would need to be dealt with. Degradation of graphite used as flow fields can result in chemical leaks which are dangerous to human life as well as the surrounding environment. Other electrodes have been tested for use in RFBs but, none have exhibited the abilities of graphite. BDD has been shown to resist fouling in high temperature, corrosive, and high potential environments [61]. Because BDD has previously not been used as an electrode for RFBs, its corrosion and fouling resistance must be examined in the environments utilized by RFBs.

To compare the corrosion resistance of BDD in a high potential and highly corrosive environment, chronoamperometry at $1.8 \mathrm{~V}$ vs. $\mathrm{Ag} / \mathrm{AgCl}$ was performed using fuel cell grade graphite as the electrode. The voltage was maintained for 10 days in an electrolyte solution consisting of $1 \mathrm{mM} \mathrm{Ce} 2\left(\mathrm{CO}_{3}\right)_{3}$ and $1 \mathrm{M} \mathrm{H}_{2} \mathrm{SO}_{4}$. The results are shown in Figure 41. Clearly, the graphite did not hold up well. After 10 days, a hole was etched straight through the graphite material. The exact same test was performed on a BDD sample. After 10 days, the BDD sample retained its integrity, shown in Figure 42. any difference in the two images are a result of a surface termination shift from the as-grown hydrogen to oxygen as well as a reduction in non-diamond carbon at the grain boundaries. It is important to note that a substantial reduction in gas evolution was observed on the BDD surface compared to the graphite surface. This indicates a significantly improved faradaic efficiency can be achieved with BDD. Figure 43 shows Raman spectroscopy testing results before and after the previously described electrochemical test. There is no significant shift in the Raman spectra before and after long-term testing, indicating the 
BDD retained its integrity. Additionally, there is no peak associated with non-diamond carbon, or $\mathrm{sp}^{2}$, before or after testing.

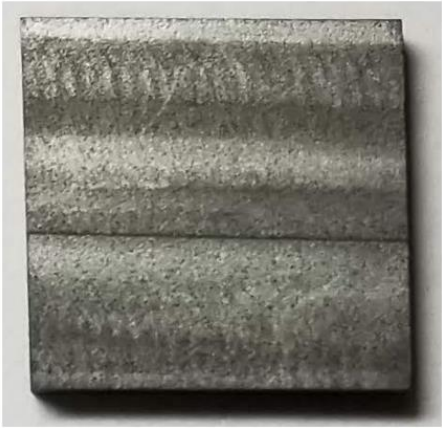

Before

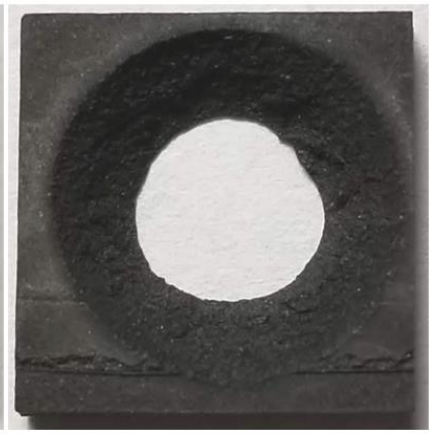

After

Figure 41. Before and after images of fuel cell grade graphite showing a hole electrochemically etched through the graphite.
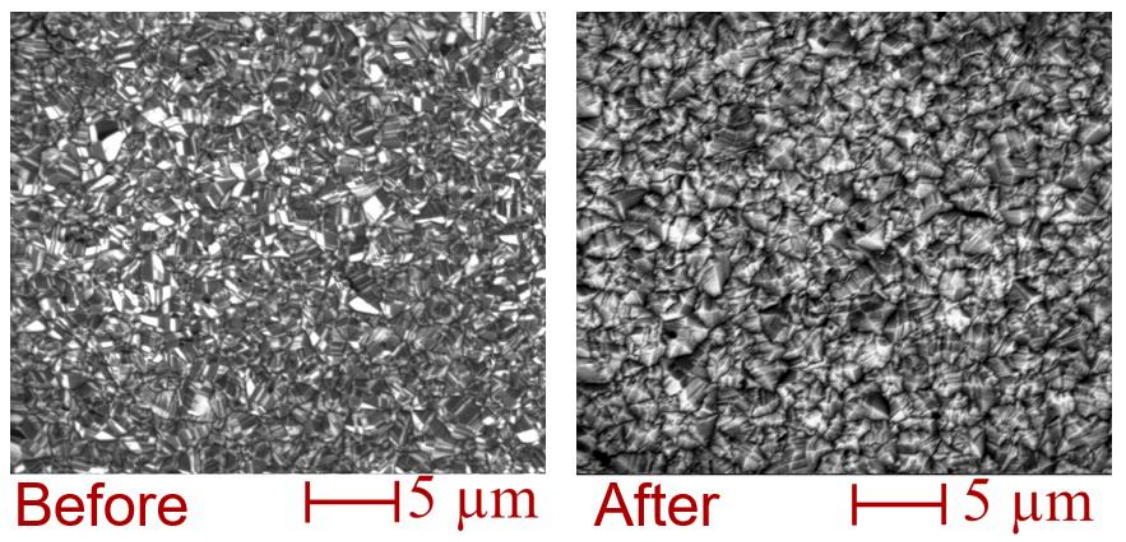

Figure 42. SEM images before and after long-term charging at high potential on BDD. 


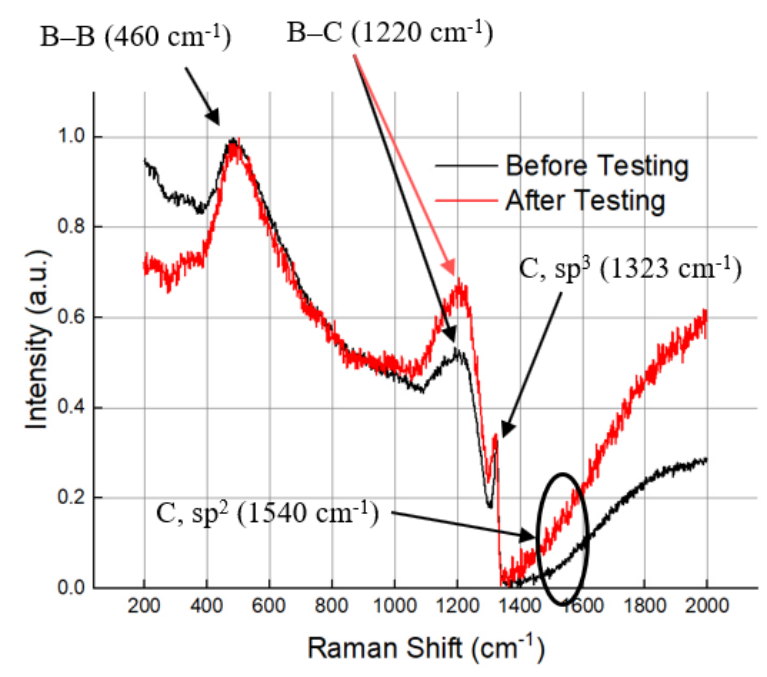

Figure 43. Raman spectra before and after long-term charging at high potential.

Cyclic voltammetry was performed in a $1 \mathrm{mM} \mathrm{Ce(NO3)3} \mathrm{and} 1 \mathrm{M}$ HNO3 solution to demonstrate the ability of BDD compared to graphite with the $\mathrm{Ce} 3+/ \mathrm{Ce} 4+$ redox couple. The oxidation and reduction peaks of this redox couple are clear, Figure 44(a), on the BDD sample. On the graphite sample, the reduction peak can be seen; however, with a higher overpotential compared to BDD (shifted more negative in potential). The oxidation peak on graphite is not clearly observable as it overlaps with $\mathrm{C}$ corrosion and gas evolution. The current density on graphite is higher compared to BDD. This is believed to be, at least in part, a result of a higher surface area, due to the surface morphology inherent in graphite. It may also indicate the reaction rate is faster on graphite compared to BDD.

Figure 44(b) shows potentiodynamic anodic polarization of BDD and graphite. There is no clear oxidation peak, expected around $1.8 \mathrm{~V}$. Instead, the current density sharply rises, beginning near $1.2 \mathrm{~V}$, because of $\mathrm{C}$ corrosion and gas evolution. The current density achieved on graphite, combined with the liberation of $\mathrm{C}$ particles observed in the electrolyte solution, indicates a significant amount of $\mathrm{C}$ corrosion and gas evolution 
occurred. The potentiodynamic anodic polarization of BDD is quite different. A clear oxidation peak is seen at about $1.7 \mathrm{~V}$ and the current density does not sharply increase until nearly $2 \mathrm{~V}$. Clearly the BDD electrode is superior in terms of corrosion and gas evolution resistance. Figure 44(c) demonstrates the increasing current density of the oxidation and reduction peaks resulting from increased concentration of active species.

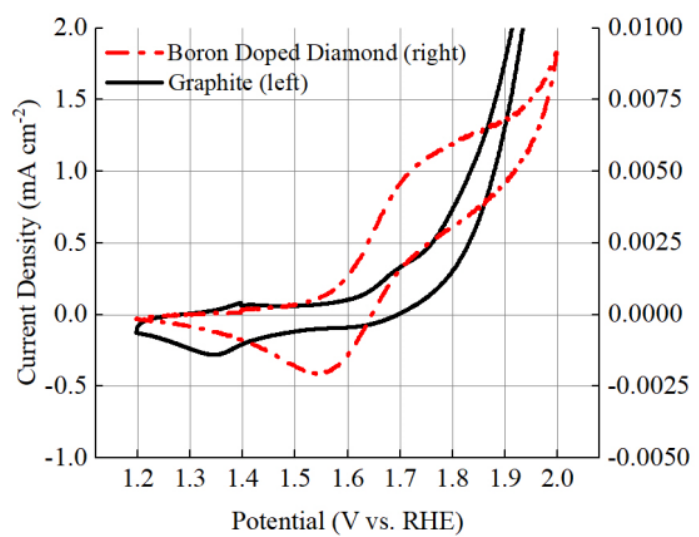

(a)

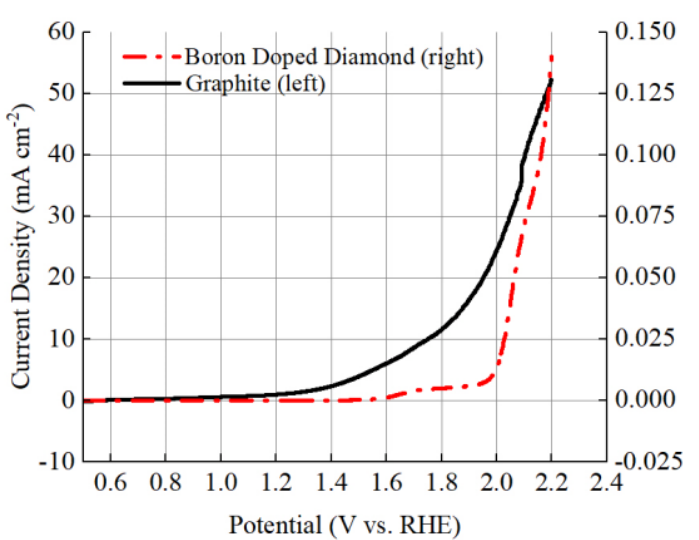

(b)

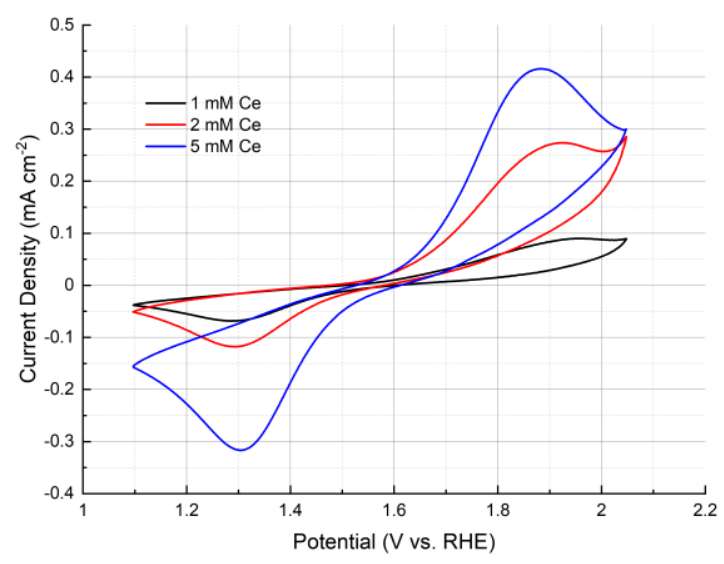

(c)

Figure $44.1 \mathrm{mM} \mathrm{Ce}\left(\mathrm{NO}_{3}\right)_{3}$ in $1 \mathrm{M} \mathrm{HNO}_{3}$ on fuel cell grade graphite and $\mathrm{BDD}$ compared using (a) cyclic voltammetry at $1 \mathrm{mV} \mathrm{s}^{-1}$, (b) potentiodynamic anodic polarization, and (c) cyclic voltammetry at different active species concentration. 
The fouling resistance of BDD is demonstrated with testing of the $\mathrm{Mn}^{2+} / \mathrm{Mn}^{3}$ redox couple. The $\mathrm{Mn}^{3+}$ ion is known to disproportionate to $\mathrm{MnO}_{2}$ spontaneously in solution. This can lead to a buildup of $\mathrm{MnO}_{2}$ on the electrode surface, clogging the electrode and preventing further reaction. Figure 45 shows CV scan of the $1^{\text {st }}, 200^{\text {th }}$, and $320^{\text {th }}$ scan of $100 \mathrm{mM} \mathrm{MnSO}{ }_{4}$ and $500 \mathrm{mM} \mathrm{H}_{2} \mathrm{SO}_{4}$ on a BDD electrode. From Figure 45, it is observed that the peak height of the $200^{\text {th }}$ scan is significantly reduced compared to the $1^{\text {st }}$ scan. This is a result of $\mathrm{MnO}_{2}$ precipitation buildup on the electrode surface. After this, the electrochemical experiment was paused, and a pipette was used to gently spray fluid across the electrode surface. The electrochemical experiment was resumed and the $320^{\text {th }}$ scan is shown in Figure 45. The peak height of oxidation and reduction not only returned to their initial position but, exceeded them indicating better reaction kinetics.

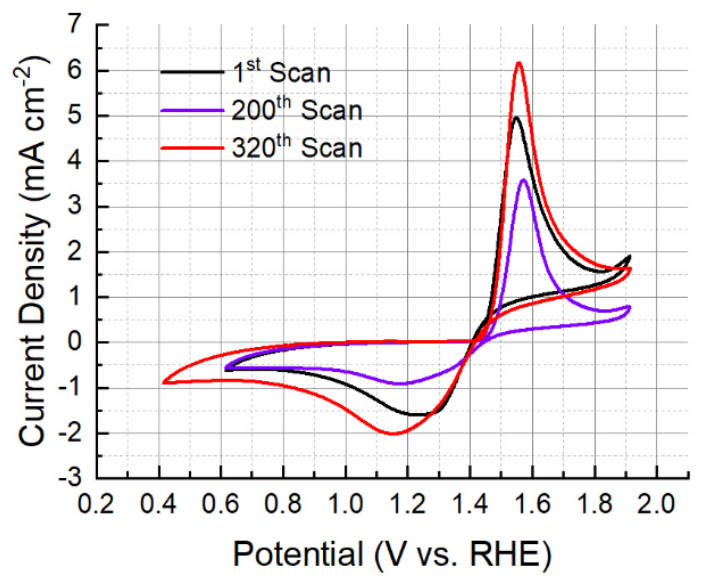

Figure 45. CV scans of $100 \mathrm{mM} \mathrm{MnSO}_{4}$ in $500 \mathrm{mM} \mathrm{H}_{2} \mathrm{SO}_{4}$ on a BDD electrode.

\subsection{Conclusion}

In this chapter, the fabrication and characterization methods for BDD are demonstrated. SEM images show highly crystalline BDD with crystal sizes on the order of 
a micron, Figure 30. SEM images indicate continuous growth of BDD thin films without the existence of pin holes which may compromise the substrate material. SEM imaging of cleaved samples indicate BDD thicknesses of hundreds of $\mathrm{nm}$ to tens of microns is easily achievable with the demonstrated fabrication technique, Figures 28 and 29. Good adherence of the BDD to silicon substrates is also demonstrated as the cleaved samples do not simply delaminate from the silicon. Instead, the silicon underneath the diamond tends to crack first, leaving some of the silicon substrate attached to the BDD layer.

Characterization by Raman indicates high quality diamond with little observance of non-diamond carbon (NDC) content. Comparison of the Raman spectra with published literature, for which the B content was quantified, shows the BDD fabricated for this dissertation is highly doped resulting in metal-like conductivity. After testing in a high potential and highly corrosive environment, Raman shows little to no diamond peak shift and the presence of NDC content is still absent, Figure 43. This illustrates the extreme corrosion resistance of BDD. In fact, SEM images before and after testing with the $\mathrm{Ce}^{2+} / \mathrm{Ce}^{3+}$ redox couples suggest the removal of any NDC content that may have been present, Figure 42. Additionally, FTIR data, Figure 32, confirms the existence of pristine BDD samples. FTIR data also shows little to no $\mathrm{N}$ impurities.

Chapter 4 demonstrates the electrochemical ability of BDD to function as an electrode using the $\mathrm{Ce}^{3+} / \mathrm{Ce}^{4+}$ and $\mathrm{Mn}^{2+} / \mathrm{Mn}^{3+}$ redox couples. The kinetics of the redox couples on BDD are shown to be comparable to the ideal catalyst, platinum. Figure 38 shows a direct comparison of BDD and platinum using the $\mathrm{Mn}^{2+} / \mathrm{Mn}^{3+}$ redox couple. The peak heights and peak separations of the BDD electrodes and platinum are very close. The peak height and peak separation are good indicators of kinetics and reversibility. Good 
reaction kinetics tends to result in higher current density at the peak and lower overpotentials, or lower peak separation.

The cycling ability of BDD is shown in Figure 45. After 320 cycles using the $\mathrm{Mn}^{2+} / \mathrm{Mn}^{3+}$ redox couple, the BDD electrode demonstrates improved performance. Additional testing further demonstrates the cycling ability of BDD and exemplifies the corrosion resistance of BDD through a long term, constant voltage charging regime. In Figure 41, the extreme degradation of a traditional carbon-based electrodes is shown by the etching of a hole completely through the material. BDD remains unaffected under the same conditions.

In this chapter, it is made clear that BDD may be applied as an electrode material in RFBs to good effect. The wide solvent window results in less observed gas evolution with no indication of corrosion. In addition, good reaction kinetics and reversibility were demonstrated. Together, the outcome of the research demonstrates an electrode with a long lifetime, extreme corrosion resistance, good reversibility and kinetics to low-cost, high potential redox couples, and high efficiency resulting from a decrease in gas evolution. 


\section{CHAPTER 5}

\section{EXPLORATION OF OTHER ECONOMICAL REDOX COUPLES ON A BDD ELECTRODE}

\subsection{Background and state-of-the-art}

The $\mathrm{I}^{-} / \mathrm{I}_{3}^{-}$redox couple is promising due to its low cost compared to vanadium and its two-electron transfer process, allowing for a higher energy density at similar solubilities. Li et al. fabricated a polysulfide/iodide redox flow battery to achieve a high energy density (43.1 $\mathrm{W} \mathrm{h} \mathrm{L}^{-1}$, based on catholyte and anolyte volume) [57]. This battery employs the $\mathrm{S}^{2-} / \mathrm{S}_{2}{ }^{2-}$ couple in the anolyte and the $\mathrm{I}^{-} / \mathrm{I}_{3}{ }^{-}$couple in the catholyte. The RFB exhibited a coulombic efficiency of $90 \%$ at a current density of $25 \mathrm{~mA} \mathrm{~cm}^{-2}$ and offers cost savings ( $\$ 85.4 \mathrm{~kW} \mathrm{~h}^{-1}$ active materials cost) when compared to traditional vanadium systems (\$152.0 $\mathrm{kW} \mathrm{h}^{-1}$ active materials cost) [57]. A Zn/I RFB was developed and tested by Zhang et al. [50]. This RFB achieved an energy density of $330.5 \mathrm{Wh} \mathrm{L}^{-1}$, far higher than any other aqueous RFB to date [50]. The standard potential for the Zn/I RFB is $1.796 \mathrm{~V}$. At $20 \mathrm{~mA} \mathrm{~cm}{ }^{-2}$, an energy efficiency of $\sim 70 \%$ was achieved [50]. The energy efficiency significantly fluctuated during operation due to dendrite formation and electrode shape change. An energy density of $200 \mathrm{Wh} \mathrm{L}^{-1}$ was maintained after $200 \mathrm{~h}$ of testing [50].

Fe redox couples, including the all-iron RFB, have been explored due to the

extreme low cost of $\mathrm{Fe}$. One of the major challenges facing the $\mathrm{Fe}^{2+} / \mathrm{Fe}^{0}$ redox couple at the anode is the evolution of hydrogen, a parasitic reaction. Zn metal is innately resistant 
to hydrogen evolution. As Zn plates at the anode, hydrogen evolution is not as much of a concern, even at the low standard potential of the $\mathrm{Zn}^{2+} / \mathrm{Zn}^{0}$ redox couple. Fe does not have this advantage. As the Fe plates, creating a solid Fe anode regardless of the starting anode material, hydrogen evolution significantly reduces coulombic efficiency. Jayathilake et al. found that by controlling the temperature and $\mathrm{pH}$ of the anolyte, the kinetics of the $\mathrm{Fe}$ deposition reaction could be improved so that it is favored over hydrogen evolution [48]. This discovery allowed for a coulombic efficiency of $97.9 \%$ at $20 \mathrm{~mA} \mathrm{~cm}{ }^{-2}$ [48]. The research also found that the codeposition of cadmium, which is immiscible with Fe, can improve coulombic efficiency due to its high overpotential to hydrogen evolution [48].

An aqueous all-iron RFB was tested by Wei et al. and achieved an energy density of $76 \%$ at $2 \mathrm{~mA} \mathrm{~cm}$ cm $^{-2}$ [100]. The expected active material cost was found to be $22 \$ \mathrm{kWh}^{-1}$, significantly less than that of vanadium at $118 \$ \mathrm{kWh}^{-1}[100]$. The anode was composed of a $\mathrm{Fe}_{3} \mathrm{O}_{4}$ nanoparticle and carbon black slurry pasted onto a nickel foam [100]. The cathode was graphite felt and both electrolyte solutions contained $3 \mathrm{M} \mathrm{KOH}$ as the supporting electrolyte [100]. Another all-iron RFB, using a non-aqueous solvent, was tested by Zhen et al. [115]. The OCV was determined to be $1.34 \mathrm{~V}$ and an energy efficiency of $83.4 \%$ was obtained at $10 \mathrm{~mA} \mathrm{~cm}{ }^{-2}$ [115]. Graphite felt electrodes were used in both half-cells [115]. Many more iterations of RFBs utilizing one or both Fe redox couples exist due to the low-cost and many oxidation states of Fe.

An all-copper RFB has been proposed and tested by Sanz et al. due to the many oxidation states of $\mathrm{Cu}$ and its reduced cost, $50 \$ \mathrm{kWh}^{-1}$ compared to vanadium [83]. The hybrid all-copper RFB achieved an energy efficiency of $93 \%$ at $5 \mathrm{~mA} \mathrm{~cm}{ }^{-2}$, reducing to $74 \%$ at $20 \mathrm{~mA} \mathrm{~cm}^{-2}$ [84]. The high solubility of copper allows for an energy density of 
$20 \mathrm{Wh} \mathrm{L}^{-1}$, comparable to the all-vanadium RFB system [84]. The supporting electrolyte was $\mathrm{HCl}$ with calcium chloride added to increase the number of free $\mathrm{Cl}$ ions [84]. Graphite electrodes were used in both half-cells [84]. A non-aqueous all-copper RFB was demonstrated by Li et al. utilizing the same redox couples as the previous examples [56]. This RFB achieved an energy efficiency of $44 \%$ at $5 \mathrm{~mA} \mathrm{~cm}^{-2}$ [56]. The challenges facing the all-copper RFB include dendrite formation, electrode shape change, low power density, and low efficiency at higher current densities.

\subsection{Rationale}

The additional redox couples explored in this chapter, each having their own set of unique problems from high gas evolution rates to low kinetics, are promising examples of low-cost redox actives species. Many of the problems inherent to each system may be solved by introducing a new electrode material. Use of a BDD electrode with these additional redox couples may expose a redox reaction with high kinetics on BDD that is low-cost. This will set the path for another avenue of exploration in RFB technology. In addition, as BDD is a new electrode to RFBs, additional information on the function of BDD is necessary to discern a method of progress. This chapter will also exhibit the superiority of BDD as a RFB in terms of its wide solvent window compared to platinum and a traditional carbon-based electrode. This further solidifies the usefulness of BDD as an electrode for RFBs. 


\subsection{Testing of various redox couples on a BDD electrode}

There is an endless supply of redox couples in the world of chemistry to explore; however, they will not all work on any electrode surface. That is to say, the reaction kinetics of some redox couples on a given electrode surface may be so small that it is regarded as non-existent. If reaction kinetics are slow, the maximum operating current density will be low. A low current density requires a larger RFB cell to produce a set amount of power output. Additionally, if the reaction is not facile on the electrode surface, large overpotentials will be required for the reaction to proceed. For a RFB to be competitive in the grid scale energy storage market, it must have a high energy efficiency. It is impossible to have a high efficiency battery when large overpotentials are present. These criteria significantly limit the field of available redox couples.

The BDD electrode provides a wide solvent window to work with. It achieves this by inhibiting the oxygen and hydrogen evolution reactions. Evolution reactions are inhibited by making it difficult for molecules to adsorb on the surface of BDD [61]. Water molecules must adsorb to the surface of an electrode for the necessary steps involved in gas evolution to take place. Similarly, many redox species must also adsorb to the surface for reaction to take place. Unfortunately, what BDD does to prevent gas evolution, it also does to other chemical species. As a result, significant reaction kinetics may only be found with redox couples that exhibit outer sphere reactions. In these reactions, the chemical species does not have to specifically adsorb to the electrode surface, electron transfer can take place over a finite distance. The key then, is to find highly reversible redox couples that can react on the BDD surface with sufficient reaction kinetics. BDD electrodes have 
never been tested on redox chemistries used for RFBs. Because of this, it is not known which redox couples will work and which will not.

Iodine has shown promise in RFB technology due to its high energy density resulting from its high solubility and two electron transfer reaction. The iodine redox couple is

$$
\mathrm{I}_{3}^{-}+2 e^{-} \rightleftharpoons 3 \mathrm{I}^{-}
$$

The electrolyte was made by dissolving $0.1 \mathrm{M}$ potassium iodide (KI) in DI water. In this way, potassium ions can act as the mobile ions, passing through the ion-exchange membrane, to balance charge in the RFB. As a result, the electrolyte solution remains neutral in $\mathrm{pH}$. This is a benefit as special materials are not required and safety and environmental concerns are lower. Figure 46 shows CV results of the iodine electrolyte on two BDD samples and a graphite rod. One BDD sample was previously used in testing with manganese and so, had been partially fouled. This resulted in much larger overpotential as compared to the graphite rod. When a fresh BDD sample was employed, BS13, the CV results compared closely with the graphite rod. 


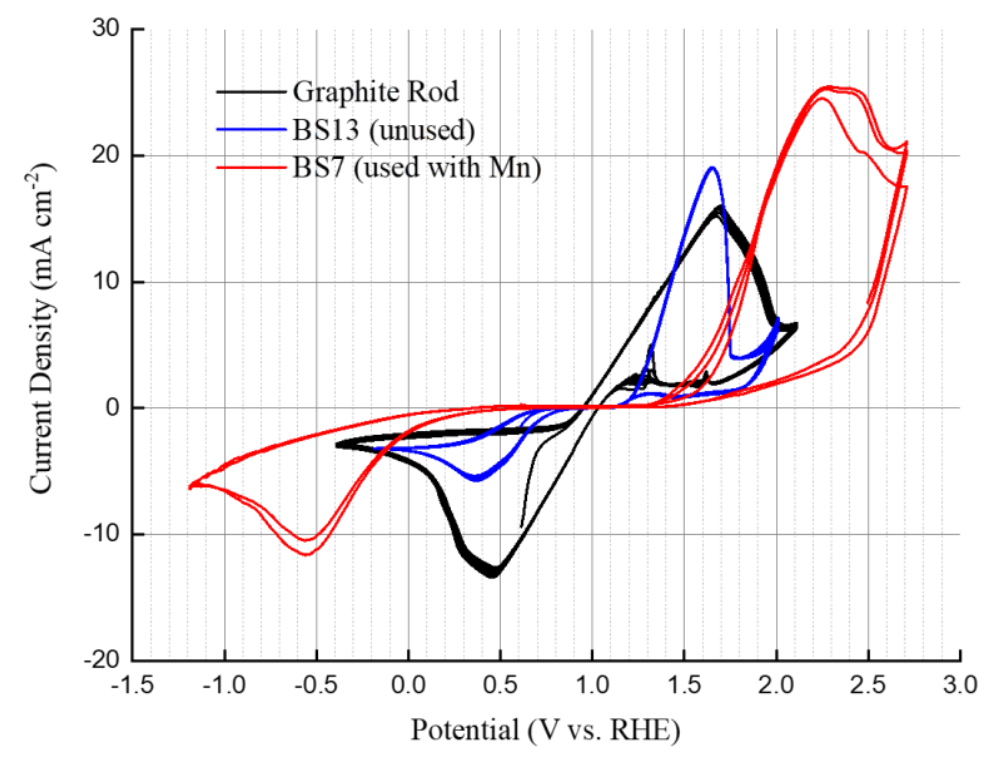

Figure 46. CV of a BDD electrode at a scan rate of $20 \mathrm{mV} \mathrm{s}^{-1}$ in an electrolyte solution consisting of $0.1 \mathrm{M}$ potassium iodide. Two BDD samples are compared, one used and the other new, along with a graphite rod.

Iron is the most abundant element on earth by mass [29]. This makes it attractive for use in energy storage as it will be cheaper than most competing options. An iron redox couple which is inside the solvent window of water is

$$
\mathrm{Fe}^{3+}+e^{-} \rightleftharpoons \mathrm{Fe}^{2+} \quad 0.771 \mathrm{~V} \text { (vs. SHE) (16) }
$$

The results of CV testing with a BDD sample on a silicon substrate is shown in Figure 47. The iron redox couple is observable through the $\mathrm{CV}$ on $\mathrm{BDD}$; however, large overpotentials are present compared to the iron redox couple on platinum wire. 


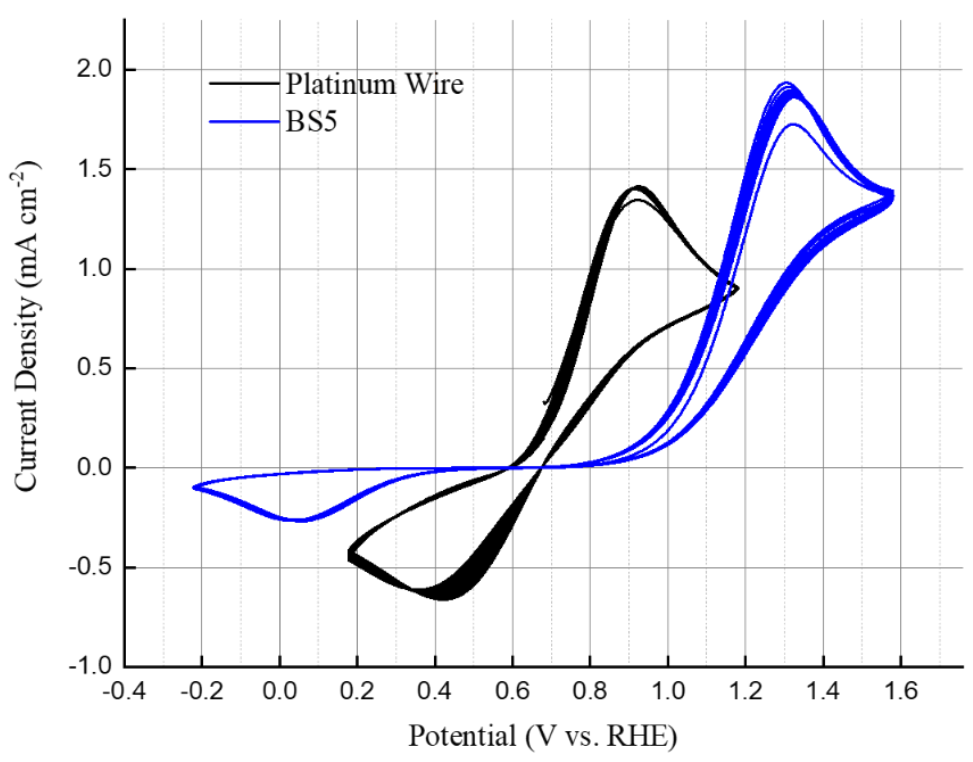

Figure 47. CV of a BDD electrode at a scan rate of $20 \mathrm{mV} \mathrm{s}^{-1}$ for platinum and $5 \mathrm{mV} \mathrm{s} \mathrm{s}^{-1}$ for the BDD electrode in an electrolyte solution consisting of $0.1 \mathrm{M}$ iron and $2 \mathrm{M}$ sulfuric acid. A BDD electrode is compare to platinum wire.

The copper redox couple

$$
\mathrm{Cu}^{2+}+e^{-} \rightleftharpoons \mathrm{Cu}^{+}
$$

potential is comparable to the vanadium anolyte redox couple. With the price of copper lower than vanadium, the copper redox couple is attractive. Additionally, there is another copper redox couple

$$
\mathrm{Cu}^{3+}+e^{-} \rightleftharpoons \mathrm{Cu}^{2+}
$$


which may be used as a catholyte redox couple and paired with the copper anolyte redox couple. The overall potential of these redox couples, based on standard potentials, is 2.247 V, much higher than the all-vanadium RFB. Additionally, the use of copper in both anolyte and catholyte offers the benefits to crossover present in the all-vanadium RFB. This make an all-copper RFB a promising endeavor to pursue. CV of the copper anolyte redox couple is shown in Figure 48. A significant shift in the CV curve exists as the cycle number increases; however, the overpotentials on BDD are reasonable considering other results presented in this study. $\mathrm{CV}$ of the copper redox couple using BDD on a silicon substrate at different scan rates is shown in Figure 49.

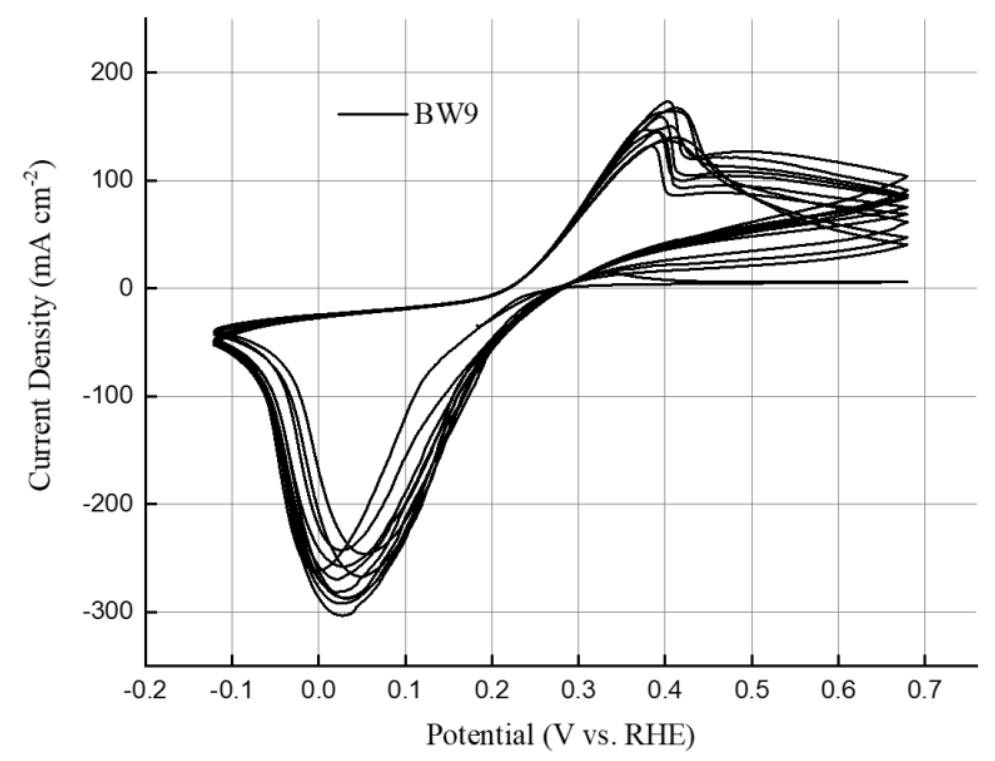

Figure 48. CV of a BDD electrode on a tungsten substrate at a scan rate of $20 \mathrm{mV} \mathrm{s}^{-1}$ in an electrolyte solution consisting of $0.1 \mathrm{M}$ copper and $2 \mathrm{M} \mathrm{H}_{2} \mathrm{SO}_{4}$. 


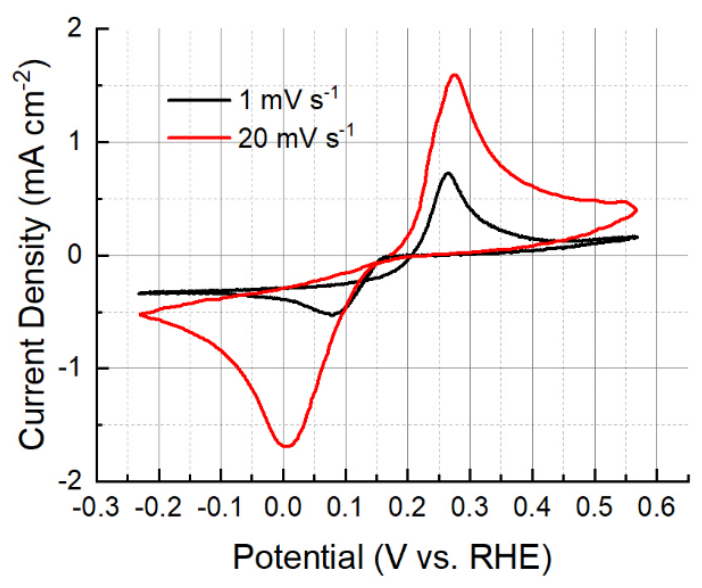

Figure 49. $\mathrm{CV}$ scans of $10 \mathrm{mM} \mathrm{CuSO}_{4}$ and $3 \mathrm{M} \mathrm{H}_{2} \mathrm{SO}_{4}$ on a BDD electrode.

In electrochemistry, the most used standard for research is the ferri/ferrocyanide redox couple

$$
\left[\mathrm{Fe}(\mathrm{CN})_{6}\right]^{3-}+e^{-} \rightleftharpoons\left[\mathrm{Fe}(\mathrm{CN})_{6}\right]^{4-} \quad 0.358 \mathrm{~V}(\text { vs. SHE) (19) }
$$

The potential of this redox couple is not desirable for RFBs but, it is a stable and highly reversible couple making it a good candidate for comparison and testing. Figure 50 shows $\mathrm{CV}$ of 500 cycles of the ferri/ferrocyanide redox couple, after performing over 1,000 cycles. The CV curves have good symmetry, low peak potential difference, and good repeatability. 


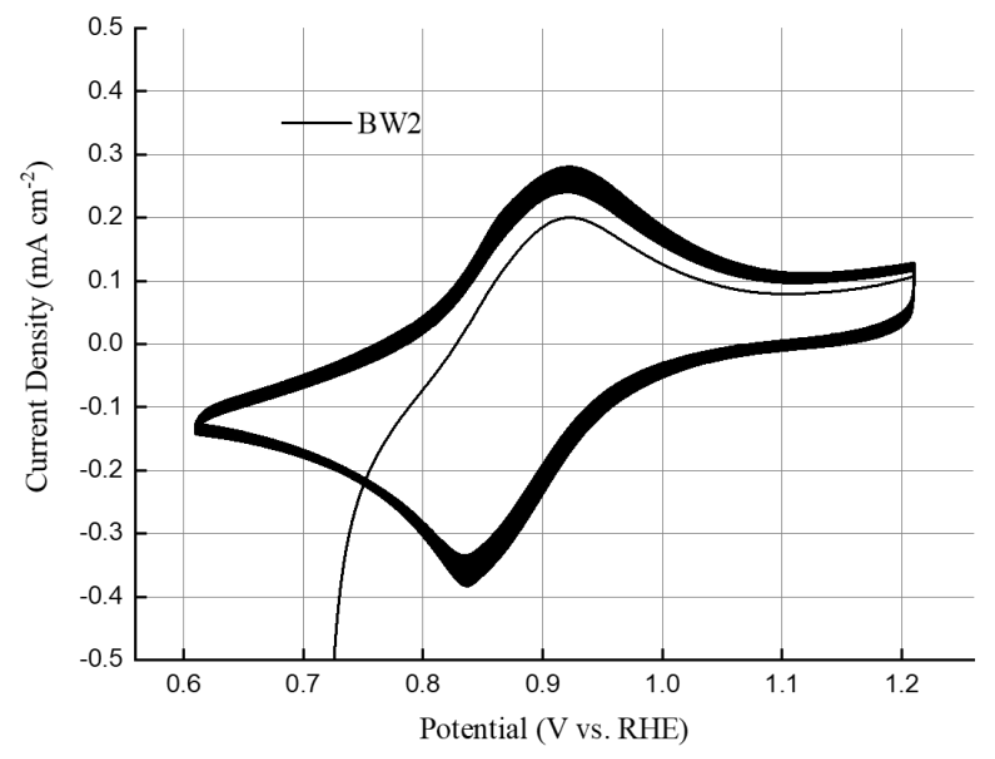

Figure 50. CV of a BDD electrode on a tungsten substrate at a scan rate of $10 \mathrm{mV} \mathrm{s}^{-1}$ in an electrolyte solution consisting of $3 \mathrm{mM}$ ferricyanide and $1 \mathrm{M}$ potassium chloride.

Tests will be continued in the same manner as previously reported in section 2.3.1. while also varying the concentration of the active species and the supporting electrolyte to determine if this plays an important role. Varying the $\mathrm{pH}$ of the electrolyte may stabilize certain redox couples, allowing for reaction to take place and be sustained; this aspect must be further examined. Reaction kinetics are highly dependent on the quality and surface functionality of the BDD electrodes. Although some success has been achieved, especially on the manganese redox couple, with a recent bad run of sample fabrication, it is difficult to strongly claim a redox couple was not effective. Retesting of redox couples will be done after better BDD electrode samples are fabricated. Additionally, there are many more redox couples available for testing than presented in this study; so, more will be tested. The efficacy of the redox couples will be determined by $\mathrm{CV}$. The peak ratio and peak difference of the CV data will show the reversibility of the redox couples on BDD. Any peak shift 
and/or introduction of new peaks during repeated CV runs will show irreversibility in the chemical species. Finally, variable scan rate $\mathrm{CV}$ will be used to determine the rate constant of viable redox couples.

There is an endless supply of redox couples in the world of chemistry to explore; however, they will not all work on any electrode surface. That is to say, the reaction kinetics of some redox couples on a given electrode surface may be so small that it can be regarded as non-existent. If reaction kinetics are slow, the maximum operating current density will be low. A low current density requires a larger RFB cell to produce a set amount of power output. Additionally, if the reaction is not facile on the electrode surface, large overpotentials will be required for the reaction to proceed. For a RFB to be competitive in the grid energy storage market, it must have a high energy efficiency. It is impossible to have a high efficiency battery when large overpotentials are present. These criteria significantly limit the field of available redox couples.

The BDD electrode provides a wide solvent window to work with. It achieves this by inhibiting the oxygen and hydrogen evolution reactions. Evolution reactions are inhibited by making it difficult for molecules to adsorb on the surface of BDD [61]. Water molecules must adsorb to the surface of an electrode for the necessary steps involved in gas evolution to take place. Similarly, many redox species must also adsorb to the surface for reaction to take place. Unfortunately, what BDD does to prevent gas evolution, it also does to other chemical species. As a result, significant reaction kinetics may only be found with redox couples that exhibit outer sphere reactions. In these reactions, the chemical species does not have to specifically adsorb to the electrode surface, electron transfer can 
take place over a finite distance. The key then, is to find highly reversible redox couples that can react on the BDD surface with enough reaction kinetics.

Figure 51 shows $\mathrm{CV}$ results of the $\mathrm{I}_{3}^{-} / \mathrm{I}^{-}$redox couple on BDD. For the $1 \mathrm{mV} \mathrm{s}^{-1}$ scan rate, the peak-to-peak separation is only $102.9 \mathrm{mV}$, approaching the ideal peak-topeak separation of $57 \mathrm{mV}$ [9]. This indicates a highly reversible redox couple.

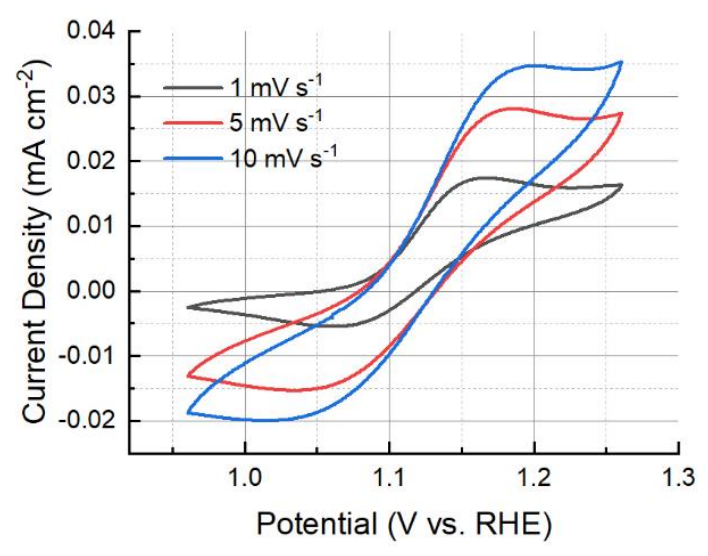

Figure 51. CV scans of $1 \mathrm{mM} \mathrm{KI}$ and $1 \mathrm{M} \mathrm{KCl}$ on a BDD electrode.

Fe is the most abundant element on earth by mass [29]. This makes it attractive for use in energy storage as it will be cheaper than most competing options. The results of CV testing with a BDD sample on a silicon substrate in a $1 \mathrm{mM} \mathrm{Fe}_{2}\left(\mathrm{SO}_{4}\right)_{3}$ and $1 \mathrm{M} \mathrm{H}_{2} \mathrm{SO}_{4}$ is shown in Figure 52. At a scan rate of $5 \mathrm{mV} \mathrm{s}^{-1}$, the peak-to-peak separation of the $\mathrm{Fe}^{3+} / \mathrm{Fe}^{2+}$ redox couple was found to be $487 \mathrm{mV}$. This peak separation is relatively high. It is likely a catalyst would need to be found to make the $\mathrm{Fe}^{3+} / \mathrm{Fe}^{2+}$ redox couple more efficiency on BDD. 


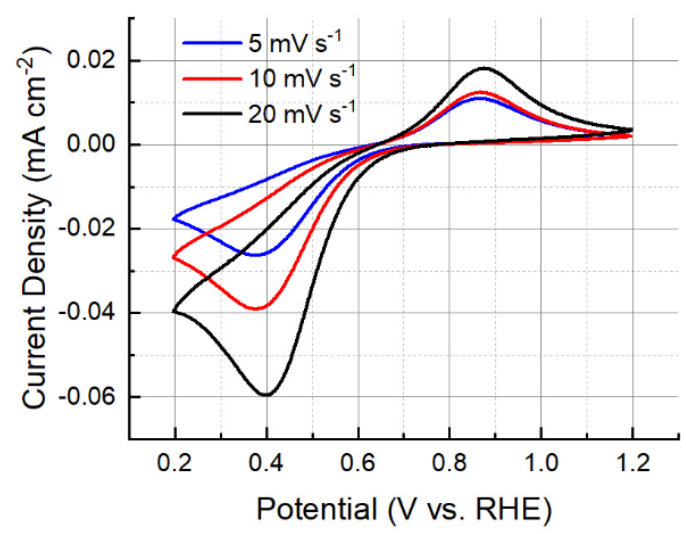

Figure 52. $\mathrm{CV}$ scans of $1 \mathrm{mM} \mathrm{Fe} 2\left(\mathrm{SO}_{4}\right)_{3}$ and $1 \mathrm{M} \mathrm{H}_{2} \mathrm{SO}_{4}$ on a BDD electrode.

Table 3 gives a summary of the values found during electrochemical experimentation on BDD compared to published literature for traditional carbon-based electrodes. 
Table 3. Comparison of electrochemical values obtained from this work and those from published literature along with operating conditions.

\begin{tabular}{|c|c|c|c|c|c|c|c|c|c|}
\hline $\begin{array}{l}\text { Redox } \\
\text { Couple }\end{array}$ & Ref. & $\begin{array}{l}\text { Working } \\
\text { Electrode }\end{array}$ & $\begin{array}{l}\text { Thermo. } \\
\text { Potential } \\
\text { (V vs. SHE) }\end{array}$ & $\begin{array}{l}\text { Formal } \\
\text { Potential } \\
(\mathrm{V} \text { vs. SHE) }\end{array}$ & $\begin{array}{l}\text { Supporting } \\
\text { Electrolyte }\end{array}$ & $\begin{array}{l}\text { Active } \\
\text { Species } \\
\text { Conc. } \\
(\mathrm{mM})\end{array}$ & $\begin{array}{l}\text { Scan Rate } \\
\left(\mathrm{mV} \mathrm{s}^{-1}\right)\end{array}$ & $\begin{array}{l}\text { Peak-to- } \\
\text { peak } \\
\text { Separation } \\
(\mathrm{mV})\end{array}$ & $\begin{array}{l}\text { Peak } \\
\text { Current } \\
\text { Ratio }\end{array}$ \\
\hline \multirow{4}{*}{$\mathrm{Mn}^{2+} / \mathrm{Mn}^{3+}$} & $\mathrm{a}$ & BDD & \multirow{4}{*}{1.51} & 1.374 & $\mathrm{H}_{2} \mathrm{SO}_{4}$ & 100 & 3 & 180.2 & 1.401 \\
\hline & $\mathrm{a}$ & $\mathrm{BDD}$ & & 1.449 & $\mathrm{HNO}_{3}$ & 10 & 1 & 232.2 & 1.123 \\
\hline & [109] & Graphite Disc & & $\sim 1.323$ & $\mathrm{H}_{2} \mathrm{SO}_{4}$ & 300 & 5 & 140 & 1.465 \\
\hline & [51] & Carbon Felt & & $\sim 1.337$ & $\mathrm{H}_{2} \mathrm{SO}_{4}$ & 1,000 & 3 & $\sim 488.8$ & $\sim 0.840$ \\
\hline \multirow{7}{*}{$\mathrm{Ce}^{3+} / \mathrm{Ce}^{4+}$} & a & BDD & \multirow{7}{*}{1.61} & 1.406 & $\mathrm{H}_{2} \mathrm{SO}_{4}$ & 1 & 1 & 254.8 & 3.671 \\
\hline & $\mathrm{a}$ & $\mathrm{BDD}$ & & 1.395 & $\mathrm{H}_{2} \mathrm{SO}_{4}$ & 10 & 1 & 308.1 & 1.864 \\
\hline & $\mathrm{a}$ & $\mathrm{BDD}$ & & 1.417 & $\mathrm{H}_{2} \mathrm{SO}_{4}$ & 100 & 1 & 350.7 & 1.173 \\
\hline & $\mathrm{a}$ & $\mathrm{BDD}$ & & 1.670 & $\mathrm{HNO}_{3}$ & 1 & 1 & 288.2 & 1.875 \\
\hline & [64] & Graphite Felt & & $\sim 1.619$ & $\mathrm{CH}_{3} \mathrm{SO}_{3} \mathrm{H}$ & 50 & 3 & $\sim 222.2$ & $\sim 0.810$ \\
\hline & [98] & Glassy Carbon & & $\sim 1.355$ & $\mathrm{H}_{2} \mathrm{SO}_{4}$ & 100 & 20 & 476 & $\sim 1.450$ \\
\hline & [68] & Pt-Ti Mesh & & $\sim 1.589$ & $\mathrm{CH}_{3} \mathrm{SO}_{3} \mathrm{H}$ & 800 & 40 & 290 & $\sim 1.821$ \\
\hline \multirow{2}{*}{$\mathrm{Cu}^{+} / \mathrm{Cu}^{2+}$} & & BDD & \multirow{2}{*}{0.153} & 0.199 & $\mathrm{H}_{2} \mathrm{SO}_{4}$ & 10 & 1 & 187.1 & 1.844 \\
\hline & [83] & Graphite Rod & & $\sim 0.570$ & $\mathrm{HCl}$ & 2,000 & 10 & $\sim 281.6$ & $\sim 0.526$ \\
\hline \multirow{2}{*}{$\mathrm{Fe}^{2+} / \mathrm{Fe}^{3+}$} & & BDD & \multirow{2}{*}{0.771} & 0.619 & $\mathrm{H}_{2} \mathrm{SO}_{4}$ & 1 & 5 & 486.9 & 0.415 \\
\hline & [116] & Glassy Carbon & & $\sim 0.678$ & $\mathrm{HCl}$ & 25 & 10 & $\sim 86.3$ & $\sim 1.555$ \\
\hline \multirow{2}{*}{$\mathrm{I}^{-} / \mathrm{I}_{3}^{-}$} & & BDD & \multirow{2}{*}{0.536} & 0.701 & $\mathrm{KCl}$ & 1 & 1 & 102.9 & 0.316 \\
\hline & [114] & Glassy Carbon & & $\sim 0.487$ & None & 100 & 50 & $\sim 1,232$ & $\sim 0.747$ \\
\hline \multirow{3}{*}{$\begin{array}{l}\mathrm{Fe}(\mathrm{CN})_{6}^{4-} \\
/ \mathrm{Fe}(\mathrm{CN})_{6}^{3-}\end{array}$} & a & $\mathrm{BDD}$ & \multirow{3}{*}{0.358} & 0.462 & $\mathrm{KCl}$ & 1 & 1 & 73.6 & 1.074 \\
\hline & $\mathrm{a}$ & BDD & & 0.478 & $\mathrm{KCl}$ & 10 & 5 & 81.3 & 0.967 \\
\hline & [33] & BDD & & $\sim 0.439$ & $\mathrm{KOH}$ & 25 & 8 & $\sim 132.9$ & $\sim 1.008$ \\
\hline
\end{tabular}

${ }^{a}$ This work 


\subsection{Electrochemical properties of BDD}

The surface functional groups present on BDD are very important to the functionality of BDD as an electrode. The surface functional groups are the bonds that attach to the dangling carbon bonds on the BDD surface, typically hydrogen and/or oxygen. A hydrogen terminated surface is hydrophobic and unstable in air. An oxygen terminated surface is hydrophilic and stable in air. Literature has also shown surface termination of BDD with nitrates and hydroxyls $[30 ; 70]$. Currently surface termination is known to significantly affect the functionally of BDD as an electrode; however, some contradiction exists among publications, and the causes and effects are not clearly understood. Because BDD has not been tested for use in RFBs, there is little known about the effects of surface termination on redox couples used in RFBs. This must be further explored to advance the utilization of BDD as a RFB electrode.

Differences in surface functionality have been observed electrochemically through changes in cyclic voltammetry from a freshly fabricated electrode to a used electrode. Also, there exist methods of electrochemical cleaning and functionalization of BDD electrodes. One of which that has been examined is, maintaining a fixed potential above the OER potential or below the HER potential for a fixed amount of time. This was observed to have a significant effect on the resulting CV scans of the solvent window.

Of the beneficial BDD characteristics presented previously, the wide solvent window is outstanding when considering its use in RFBs. Some redox chemistries tested for RFBs resulted in low energy efficiencies due to high rates of gas evolution; BDD electrodes make the reexamination of those redox chemistries possible. The wide solvent window also allows for consideration of redox couples previously untested for use in RFBs 
due to their unacceptably high or low potential. A comparison of the solvent window for BDD (fabricated at the University of Louisville), glassy carbon (similar in function to graphite), and platinum electrodes is shown in Figure 53. The comparison was made by running several $\mathrm{CV}$ cycles with each electrode in $0.5 \mathrm{M} \mathrm{H}_{2} \mathrm{SO}_{4}$. It is clear from Figure 53 that BDD outperforms glassy carbon and platinum foil in terms of overpotentials to gas evolution.

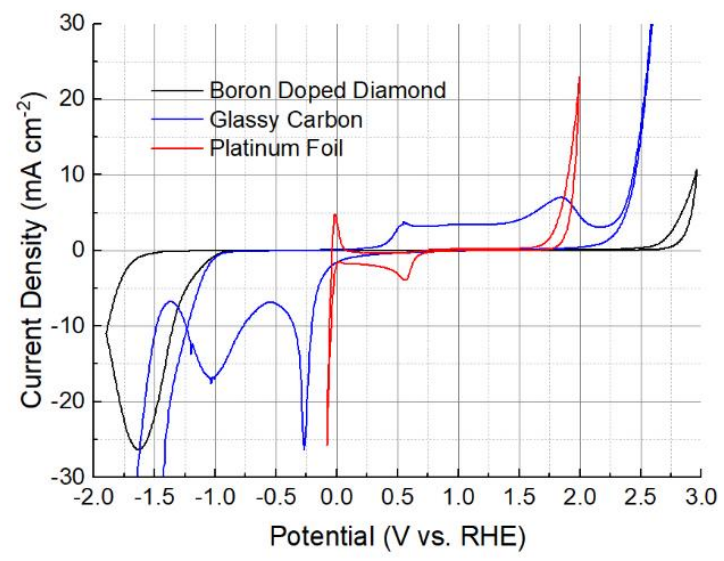

Figure 53. Solvent window comparison of BDD, glassy carbon, and platinum electrodes by $\mathrm{CV}$ in $0.5 \mathrm{M} \mathrm{H}_{2} \mathrm{SO}_{4}$.

In addition to the solvent window, electrochemical testing can determine efficiencies, reaction kinetics, reversibility, and resistances. Coulombic, voltage, and energy efficiency will be determined through charge/discharge testing of a full cell setup; a test involving both half-cells of the RFB. Electrochemical impedance spectroscopy (EIS) will be used to obtain ohmic and charge-transfer resistance. Reversibility of each redox couple was explored through $\mathrm{CV}$ by evaluating the oxidation and reduction peak difference and peak ratio. The peak difference is a measure of overpotentials present as a result of the 
electrode and active species interface. The peak ratio establishes the reversibility of the system, with a peak ratio of one being desired.

Figure 54 shows the Randles-Sevcik plot for $50 \mathrm{~mm} \mathrm{Ce}\left(\mathrm{CO}_{3}\right)_{3}$ and $50 \mathrm{~mm}$ $\mathrm{Ce}\left(\mathrm{SO}_{4}\right)_{2}$ in $1 \mathrm{M} \mathrm{H}_{2} \mathrm{SO}_{4}$. The data follows a linear trend for anodic and cathodic peak currents. This linear relationship indicates the CV scans are undergoing a diffusioncontrolled process. Diffusion values obtained using the Randles-Sevcik equation are shown in Table 4 and are consistent with published literature [98]. Diffusion coefficients for other electrolyte compositions involving the $\mathrm{Ce}^{3+} / \mathrm{Ce}^{4+}$ redox couple and the $\left[\mathrm{Fe}(\mathrm{CN})_{6}\right]^{4-} /\left[\mathrm{Fe}(\mathrm{CN})_{6}\right]^{3-}$ redox couple are also shown in Table 4.

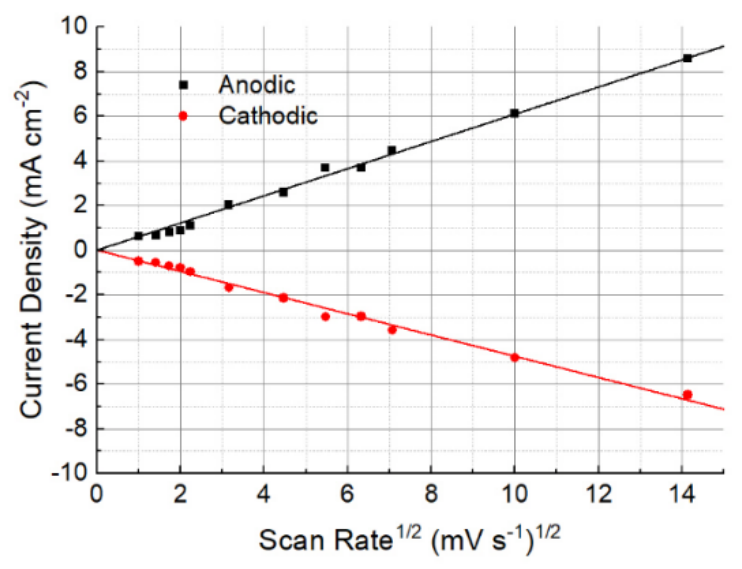

Figure 54. Current density versus the square root of scan rate for $50 \mathrm{mM} \mathrm{Ce}_{2}\left(\mathrm{CO}_{3}\right)_{3}$ and $50 \mathrm{mM} \mathrm{Ce}\left(\mathrm{SO}_{4}\right)_{2}$ in $1 \mathrm{M} \mathrm{H}_{2} \mathrm{SO}_{4}$. 
Table 4. Diffusion coefficients for cerium and $\mathrm{Fe}(\mathrm{CN})_{6}$.

\begin{tabular}{ccc}
\hline Electrolyte Configuration & Reaction Type & Diffusion Coefficient $\left(\mathrm{cm}^{2} / \mathrm{s}\right)$ \\
\hline $1 \mathrm{mM} \mathrm{Ce}\left(\mathrm{NO}_{3}\right)_{3}$ in $1 \mathrm{M} \mathrm{H}_{2} \mathrm{SO}_{4}$ & Oxidation & $8.22 \mathrm{E}-07$ \\
& Reduction & $4.01 \mathrm{E}-07$ \\
$10 \mathrm{mM} \mathrm{Ce}\left(\mathrm{NO}_{3}\right)_{3}$ in $1 \mathrm{M} \mathrm{H}_{2} \mathrm{SO}_{4}$ & Oxidation & $6.48 \mathrm{E}-07$ \\
$50 \mathrm{mM} \mathrm{Ce}_{2}\left(\mathrm{CO}_{3}\right)_{3}$ and & Reduction & $3.56 \mathrm{E}-07$ \\
$50 \mathrm{mM} \mathrm{Ce}\left(\mathrm{SO}_{4}\right)_{2}$ in $1 \mathrm{M} \mathrm{H}_{2} \mathrm{SO}_{4}$ & Oxidation & $5.14 \mathrm{E}-07$ \\
$1 \mathrm{mM} \mathrm{Ce}\left(\mathrm{NO}_{3}\right)_{3}$ in $1 \mathrm{M} \mathrm{HNO}_{3}$ & Reduction & $3.13 \mathrm{E}-07$ \\
& Oxidation & $2.14 \mathrm{E}-06$ \\
$1 \mathrm{mM} \mathrm{Fe}(\mathrm{CN})_{6}$ in $1 \mathrm{M} \mathrm{KCl}^{2}$ & Oxidation & $1.03 \mathrm{E}-06$ \\
$10 \mathrm{mM} \mathrm{Fe}(\mathrm{CN})_{6}$ in $1 \mathrm{M} \mathrm{KCl}$ & Reduction & $1.12 \mathrm{E}-06$ \\
& Oxidation & $1.70 \mathrm{E}-06$ \\
\hline
\end{tabular}

\subsection{Conclusion}

This chapter demonstrates the ability of BDD as an electrode for RFBs based on its wide solvent window and reaction kinetics for various redox couples. The wide solvent window was determined electrochemically and shown in Figure 52. Glassy carbon was chosen as it represents the widest solvent window capability of traditional carbon-based electrodes. BDD demonstrated a $>0.5 \mathrm{~V}$ increase in the positive potential of oxygen evolution and significantly reduced side reactions compared to glassy carbon. This represents the potential for high efficiency based on low gas evolution and low parasitic currents.

The ability of BDD as an electrode was demonstrated with various redox couples. Testing with the $\left[\mathrm{Fe}(\mathrm{CN})_{6}\right]^{4-} /\left[\mathrm{Fe}(\mathrm{CN})_{6}\right]^{3-}$ redox couple shows that BDD can achieve an almost ideal reversibility with very low overpotentials. With this redox couple, BDD 
showed a peak-to-peak separation of only $73.6 \mathrm{mV}$ with a peak current ratio of 1.074. These results suggest BDD may be an ideal electrode in terms of reaction kinetics and reversibility for other redox couples.

BDD also showed low peak-to-peak separation compared to literature values for the $\mathrm{Cu}^{+} / \mathrm{Cu}^{2+}, \mathrm{Fe}^{2+} / \mathrm{Fe}^{3+}$, and $\mathrm{I}^{-} / \mathrm{I}_{3}{ }^{-}$redox couples as shown in Table 3. Of particular interest is the $\mathrm{I}^{-} / \mathrm{I}_{3}{ }^{-}$redox couple. With this redox couple, $\mathrm{BDD}$ resulted in a peak-to-peak separation of only $102.9 \mathrm{mV}$ where the published literature value was $1,232 \mathrm{mV}$ on glassy carbon [114]. This result indicates the reaction is facile for the $\mathrm{I}^{-} / \mathrm{I}_{3}{ }^{-}$redox couple on the $\mathrm{BDD}$ surface. Given the high energy density of I due to its superior solubility and two electron transfer, this active species may exceed the performance of other aqueous redox couples when used in conjunction with BDD. 


\section{CHAPTER 6}

\section{SCALABILITY DEMONSTRATION OF BDD AS AN ELECTRODE FOR RFBS}

\subsection{Background and state-of-the-art}

RFBs require high surface area electrodes to enable high operating current density which results in high power density. High power density reduces the required system size and thus, capital cost. There are several methods to increase the surface area of BDD including deposition on high surface area substrates and etching of BDD films. Glesenar et al. deposited BDD on a tungsten mesh, with a thickness of 35 to $40 \mu \mathrm{m}$, using hot filament CVD [32]. The BDD quality was verified by SEM and Raman. Almeida et al. coated carbon fibers with BDD, using hot filament CVD [1]. Carbon cloths were coated with BDD by Silva et al. using hot filament CVD, resulting in capacitance values 180 times higher than on a 2D Si substrate [86]. Shi et al. increased BDD surface area by catalytic etching using electrodeposited Ni nanoparticles and a hydrogen-argon plasma [85]. This method resulted in capacitance values 22 times higher than BDD on a 2D Si substrate [85].

\subsection{Rationale}

For BDD to be utilized in RFBs for grid energy storage, a high-power density must be achieved. This requires a high current density. $\mathrm{BDD}$ is primarily grown on 2D structures 
resulting in a limited surface area; however, a high surface area is necessary to allow for high current densities. Previous methods have shown the ability of BDD to be grown on 3D substrates such as a tungsten mesh or carbon cloth. This significantly increases the electrodes surface area; however, under compression, some of the BDD layer my break, exposing the substrate. Current research involving growth of BDD on a 3D substrate, utilizes a conductive substrate. If this substrate is exposed in a RFB, the electrochemical reaction my preferentially occur there, limiting the corrosion resistance capabilities of the BDD layer. Etching methods may be preferable in this regard; however, they do not produce the high surface area of growth on a 3D substrate. As a result, etching methods may not allow for enough current density. In addition, etching methods will likely be substantially more expensive due to the growth thickness required and the additional fabrication steps.

BDD has never been tested for use in a RFB so, a novel testing apparatus must be fabricated that can employ the BDD samples for full cell testing. With full cell testing, determination of voltage, coulombic, and energy efficiency are enabled.

\subsection{Utilize a full cell testing setup to demonstrate the capability of BDD}

The next logical step in the demonstration of BDD as an electrode for RFBs is its utilization in a full flow battery configuration. To achieve that, an electrochemical cell was designed, shown in Figure 55, to contain two electrodes, pockets for fluid flow, and separation by an ion-exchange membrane. The flow battery consists of two PVC endplates and two PVC flow plates. In the flow plates, a hole is cut through the thickness allowing for fluid flow in and out. An additional pocket is cut in the center of the flow plate allowing 
for a region of fluid to accumulate and flow. At the center of the flow plate is a hole which will contain the BDD surface to act as the RFB electrode. Viton gaskets will be used to seal the electrodes as well as the membrane. Copper current collectors will contact the electrodes on the outside. This configuration will allow for an $\mathrm{In} / \mathrm{Ga}$ eutectic to reside between the BDD electrode and copper current collector, forming ohmic contact with the conductive silicon substrate.
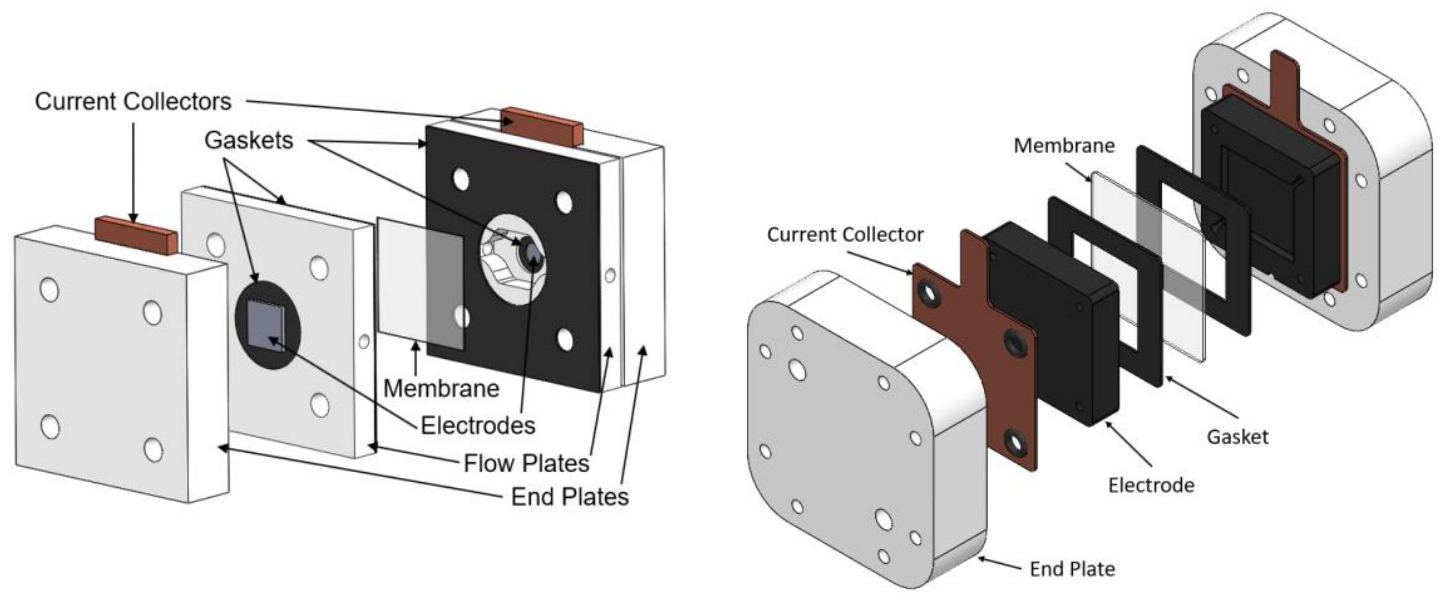

(a)

(b)

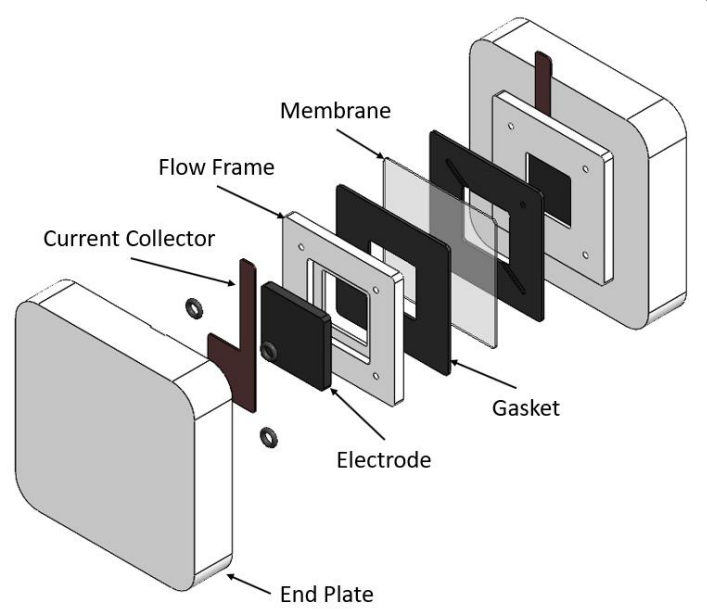

(c)

Figure 55. Evolution of fuel cell testing configurations using a (a) 2D BDD sample on a silicon substrate, (b) BDD grown on a porous graphite substrate with a CNC milled pocket or channels, and (c) BDD grown on a 2D but, porous graphite substrate. 
Testing of the BDD in the full RFB configuration will allow for the attainment of coulombic, voltage, and energy efficiencies. Energy efficiency is of key importance in RFBs as it tends to determine the cost effectiveness of the RFB system. Additionally, the full RFB setup will allow for demonstration of the corrosion resistance of BDD when cycled with a high potential redox couple such as $\mathrm{Mn}^{2+} / \mathrm{Mn}^{3+}$ or $\mathrm{Ce}^{3+} / \mathrm{Ce}^{4+}$. One of the electrodes can easily be replaced with a $2 \mathrm{D}$ graphite electrode surface so that a direct comparison can be made between BDD and a traditional graphite electrode.

\subsection{Modify the BDD electrode to increase surface area}

One of the benefits of the use of graphite as an electrode material is its ability to be fabricated with any thickness and high porosity, providing high surface area. A high surface area results in many sites for reaction to take place, allowing for charge and discharge at higher current densities. With a limited surface area, current densities will remain low and cell sizes must increase substantially to achieve a desired power output. As a result, exploration of methods to increase the surface area of BDD electrodes is desired. There are many possible methods; however, three methods, which are within the capabilities available at the University of Louisville, include growth of BDD on silicon nanowires, growth on a porous substrate such as nickel foam, and selective etching of the BDD surface. Growth of BDD thin films on silicon nanowires offers a substantial increase in surface area and this growth has been demonstrated in previous publications [74]. Growth of BDD on nickel foam is promising as this can mimic the current functionality of graphite felt electrodes [91]. Etching of BDD has been shown in create porosity and increase surface area [85]. Etching is an encouraging method because with it, complete coverage of BDD 
is insured. With growth of BDD on silicon nanowires or nickel foam, obtaining coverage on all surfaces is difficult. If only partial coverage is achieved, the acidic electrolyte in an electrochemically charged environment will attack the substrate resulting in a damaged electrode. Exploration of methods to increase the surface area of BDD is a necessary step in the use of BDD as an electrode for electrochemical battery systems.

There are many possible methods; however, three methods which are within the capabilities of the University of Louisville include growth of BDD on silicon nanowires, growth on a porous substrate such as nickel foam, and selective etching of the BDD surface. Growth of BDD thin films on silicon nanowires offers a substantial increase in surface area while maintaining a similar volume and this growth has been demonstrated in previous publications [74]. Growth of BDD on nickel foam is promising as this can mimic the current functionality of graphite felt electrodes [91]. Etching of BDD has been shown in create porosity and increase surface area [85]. This is a promising method because with it, complete coverage of BDD is insured. With growth of BDD on silicon nanowires or nickel foam, complete coverage will be difficult. If only partial coverage is achieved, the acidic electrolyte in an electrochemically charged environment will attack the substrate resulting in a damaged electrode. Sample morphology and the presence of pin holes will be determined by SEM. Brunauer-Emmett-Teller (BET) theory will be used to determine the specific surface area of samples. Finally, electrochemical methods will be used to determine the solvent window and ability of the electrode with a give redox couple.

One of the benefits of the use of graphite as an electrode material is its ability to be fabricated with any thickness and high porosity, providing high surface area. A high surface area results in many sites for reaction to take place, allowing for charge and discharge at 
higher current densities. With a limited surface area, current densities will remain low and cell sizes must increase substantially to achieve a desired power output. As a result, exploration of methods to increase the surface area of BDD electrodes is desired. There are many possible methods; however, one method that will be explored in this work is the growth of BDD on silicon on a porous graphite substrate. BDD growth on a porous graphite substrate with serpentine channels is shown in Figure 56. This plate can be assembled in a full RFB assembly to test its ability as a RFB electrode. An identical electrode can be made without BDD to be used as a standard for comparison. Figure 57 shows SEM images of the graphite flow plate with BDD grown on top. It is seen that the BDD growth is continuous on the tops of the ribs, inside the channels, and deep in the pores that reside inside the channels. Complete coverage of the graphite substrate with BDD allows for the avoidance of carbon corrosion that results in graphite at high potentials. The growth of BDD on the porous graphite surface with serpentine channels significant increases the available number of reaction sites compared to the $2 \mathrm{D}$ surface that exists on a silicon substrate. Exploration of methods to increase the surface area of BDD is a necessary step in the scale-up of BDD as an electrode for electrochemical battery systems. 


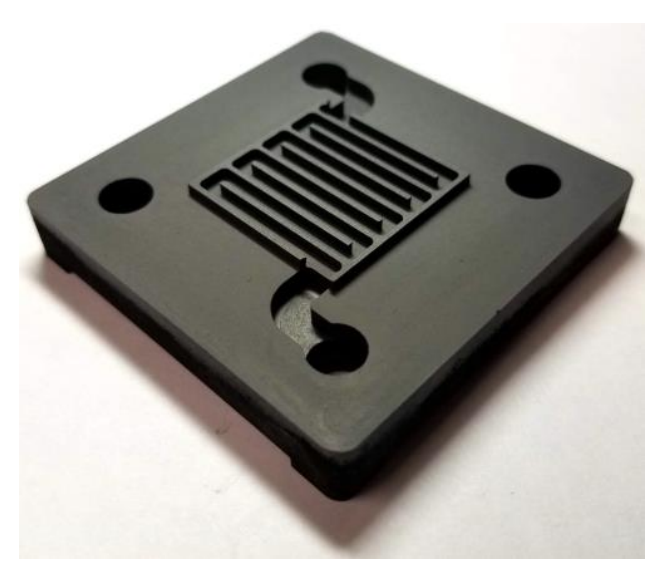

Figure 56. BDD grown on a porous graphite substrate with a serpentine channel.

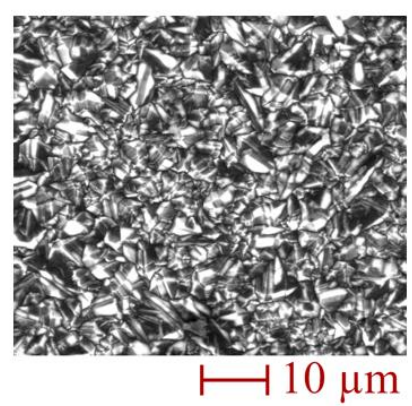

Channel ribs

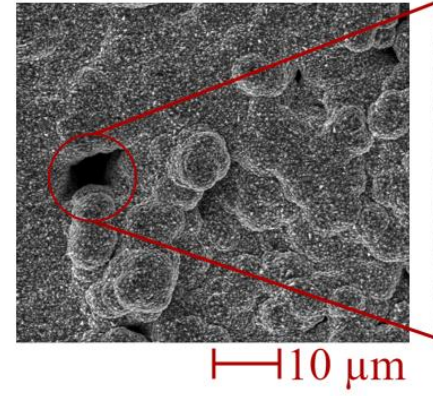

Inside channels

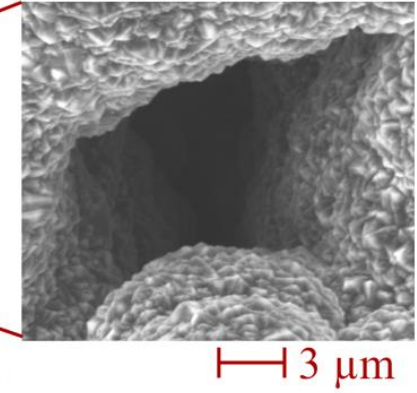

Inside a pore, inside a channel

Figure 57. SEM images of BDD grown on a porous graphite plate showing growth of diamond on the top of the serpentine channels, inside the channels, and inside a pore in a channel.

Another method for increasing the porosity of a BDD surface is changing the growth recipe. It was found, using a high-pressure recipe might promote ball-like structures of BDD, shown in Figure 58. These structures have a higher surface area then a 2D flat surface and result in a porous surface. In addition, this recipe promotes the growth of the diamond 100 plane, indicated by the square crystals in the middle image of Figure 58. Currently, it is unknown if the diamond plane orientation influences electrochemistry. This 
growth recipe must be further explored to determine its effectiveness. There is a balance between promoting porous structures and allowing for leakage pathways. Also, the recipe used in this study resulted in non-uniform growth. As seen in the right image of Figure 58, growth at a different location on the same substrate resulted in non-porous, continuous 111 diamond.
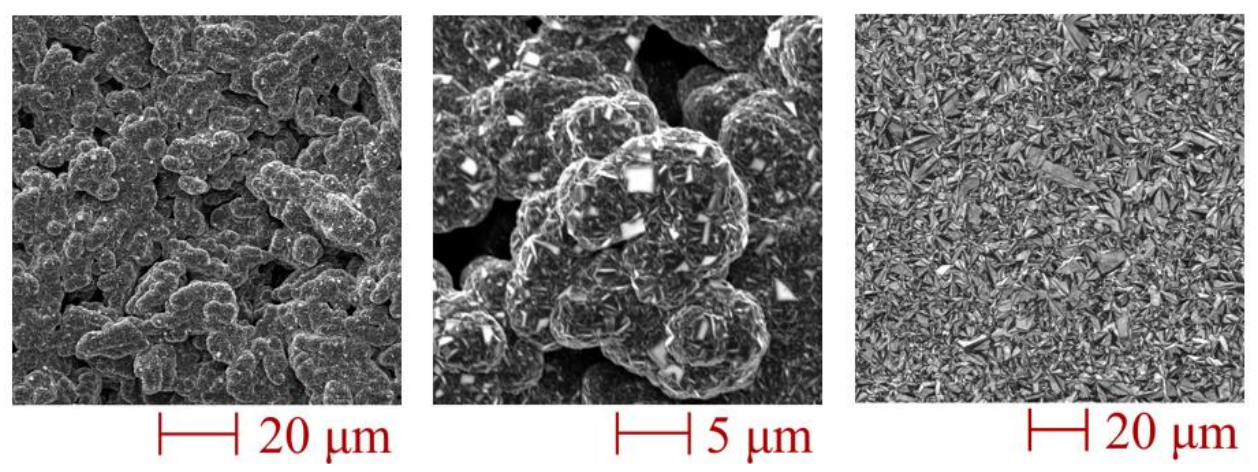

Figure 58. Growth of BDD on a porous graphite substrate using a high-pressure growth recipe.

Following in the vein of recent research, BDD growth on quartz fibers was attempted. Figure 59 shows SEM images of BDD growth on quartz wool. The left image is of bare quartz wool and the middle image of quartz wool after BDD growth, at the same magnification. It is clear, BDD has grown and significantly increased the diameter of the quartz fibers. The diamond crystal sizes remain small. Testing with a multimeter indicates low resistance in the BDD coated fibers, in the 100s of ohms range. Growing BDD on fibers with plasma is complicated by the concentration variation of radicals penetrating the fiber matrix. A systemic growth regime must be developed to promote continuous and uniform growth of BDD on a network of fibers. Other research has shown growth on fibers 
of carbon or graphite. This has the drawback of allowing exposed non-diamond carbon to react with the active species present, as opposed to reaction on BDD. This negates the benefits of the BDD. As the quartz fibers are non-conductive, reaction should be isolated to only the BDD surface.
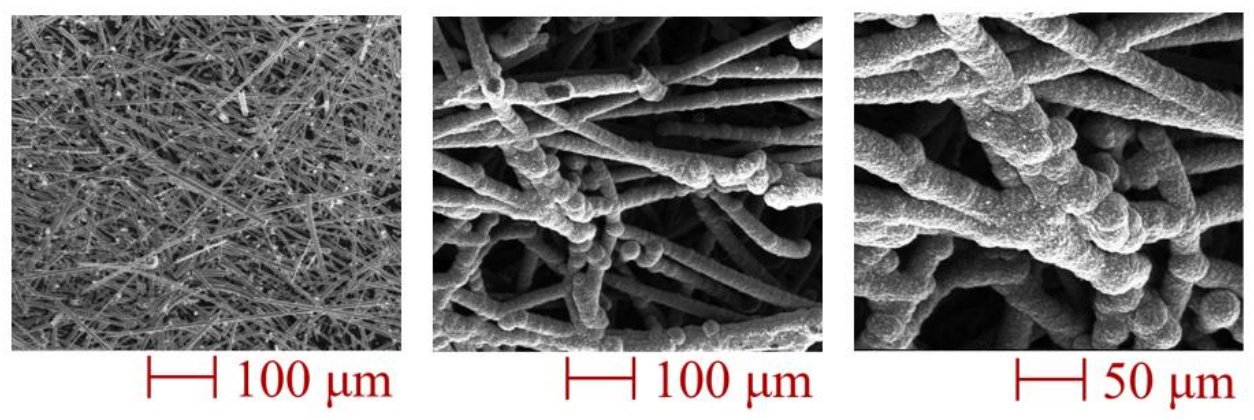

Figure 59. BDD growth on quartz wool. The first image is quartz wool without any diamond growth. The middle and right images have significant diamond growth.

BDD was also grown on quartz felt. Quartz felt and quartz wool are nearly the same thing; however, quartz felt includes a starch binder to stabilize the sheet structure, making it easier to form and cut. As Figure 60 indicates, this binder effects the morphology of the BDD growth. Figure 61(a) shows a Raman spectrum of the BDD grown on quartz felt, Figure 60. The Raman spectrum shows a diamond, $\mathrm{sp}^{3}$ peak at $1332 \mathrm{~cm}^{-1}$, along with small peaks at 460 and $1220 \mathrm{~cm}^{-1}$ representing boron doping. A sharp peak also exists at $1580 \mathrm{~cm}^{-1}$, corresponding the $\mathrm{sp}^{2}$ or non-diamond carbon. The presence of non-diamond carbon is not ideal. To improve this, adjustment of the growth recipe and/or growth regime. $\mathrm{CV}$ was performed on the BDD coated quartz felt and compared to a platinum wire in $1 \mathrm{mM} \mathrm{Ce}\left(\mathrm{NO}_{3}\right)_{3}$ and $2 \mathrm{M} \mathrm{HNO}_{3}$ electrolyte solution at $1 \mathrm{mV}^{-1}$. A significant amount of gas evolution occurred on both structures. The key feature in this CV plot is the comparison 
of current density. BDD on quartz has a much higher current density than the platinum wire, indicating the presence of significantly more surface area. No peak is observed for the $\mathrm{Ce}^{3+} / \mathrm{Ce}^{4+}$ redox couple, a result of the non-diamond carbon present. The $\mathrm{Ce}^{3+} / \mathrm{Ce}^{4+}$ redox reaction is in competition with gas evolution and carbon corrosion so, the peak is hidden.
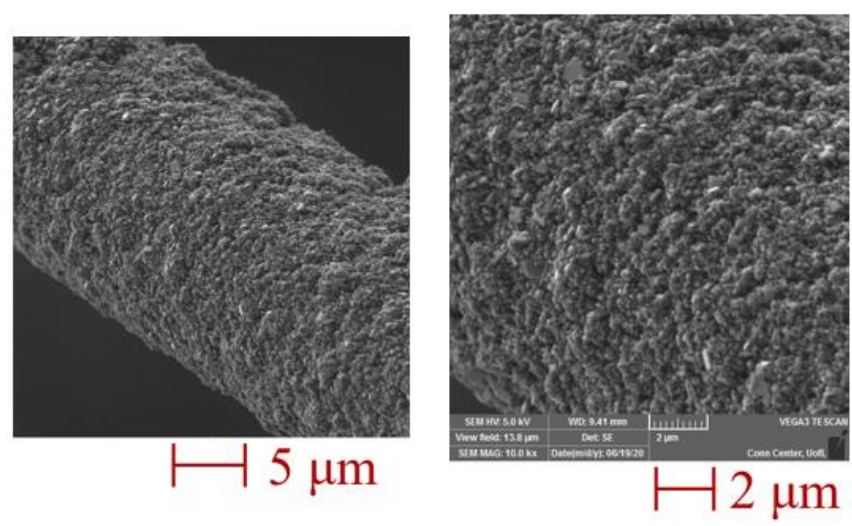

Figure 60. BDD growth on quartz felt.

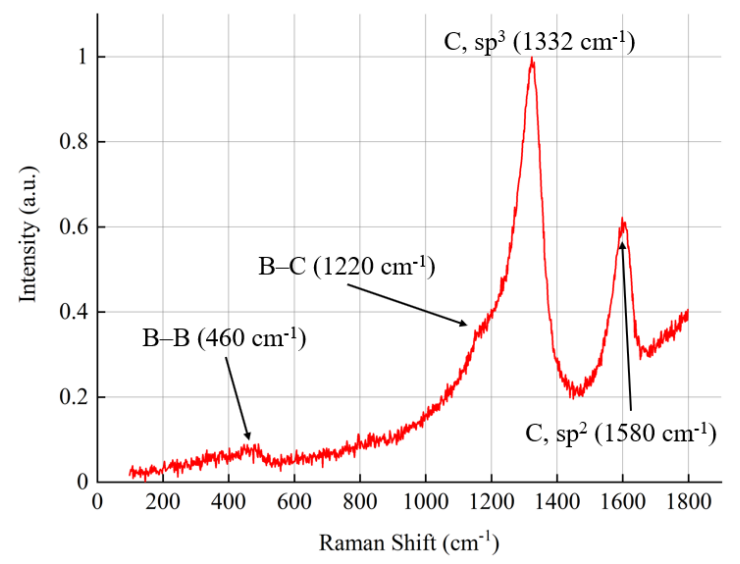

(a)

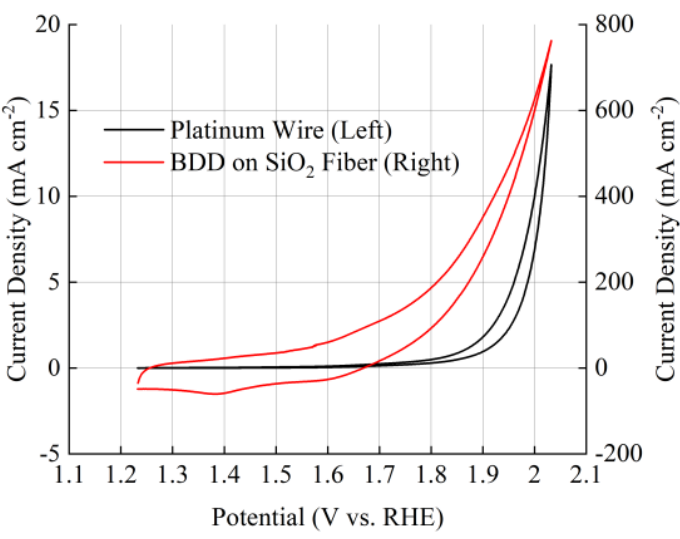

(b)

Figure 61. (a) Raman spectra of BDD grown on quartz felt and (b) CV of $1 \mathrm{mM} \mathrm{Ce}\left(\mathrm{NO}_{3}\right)_{3}$ in $2 \mathrm{M} \mathrm{HNO}_{3}$ at $1 \mathrm{mV} \mathrm{s}^{-1}$ on platinum wire and BDD on quartz felt. 


\subsection{Conclusion}

Increased surface area was achieved by growth of BDD on a porous substrate with CNC milled channels. Channels have been shown to increase RFB efficiency and operating current density by better distributing the electrolyte solution across the BDD surface. The ability to grow BDD on graphite is promising as graphite has good mechanical strength in compression and is easy to machine. Graphite plates can also be fabricated in a mold under high pressure. This method of fabrication for graphite plates combined with hot filament CVD of BDD suggests cost effective manufacturing is possible.

Growth of 3D structures was also shown to be possible using a specific growth recipe and by growth on quartz fibers. These 3D structures can result in increased surface area, which is necessary to confirm the viability of BDD as an electrode for RFBs. Increased surface area was demonstrated with BDD on quartz felt compared to a platinum wire. 


\section{CHAPTER 7}

\section{CONCLUSION}

The research presented in this dissertation demonstrates that BDD is a viable electrode in a RFB environment with several advantages over traditional carbon-based electrodes when operating at high potentials. This is important because there exist several high potential redox couples, that are low-cost compared to vanadium but are not practical on traditional carbon-based electrodes. At high potentials, BDD does not show signs of corrosion and exhibits significant reduction in gas evolution. Reduced corrosion leads to increased longevity and coulombic efficiency. Reduction in gas evolution also leads to coulombic efficiency gains, as this reaction is in kinetic competition with the redox reactions of the active species. Operating at higher potentials is beneficial as it can result in higher energy and/or power densities. Increasing either energy or power density leads to reduced system costs through a reduction in the required electrolyte volume and/or electrochemical cell size/number.

Other redox active species were demonstrated on BDD. The extremely low overpotential and high reversibility of ferri/ferrocyanide on BDD indicates there exists redox couples with great performance characteristics on BDD. The results using iodine area also intriguing as the overpotential for those redox reactions on BDD are low compared to a traditional carbon-based electrode. This shows improvement over traditional carbon-based electrodes may be realized even without a high potential redox couple. Iodine 
is a high energy density alternative active species that can operate in a neutral solution, leading to additional cost reduction in system material constraints.

Potential pathways to scale up of the BDD to a full RFB were demonstrated. To utilize BDD in a full cell RFB, as indicated by findings herein, surface area must be increased. Surface area can be increased through specific adjustment of the growth recipe, as shown, such as creating 3D structural morphologies. Growth of BDD on quartz fiber substrates was also demonstrated. Quartz offers advantages are carbon fiber substrates because it is non-conductive. In a full cell RFB configuration, due to compression, it is inevitable that the substrate will be exposed. If a carbon substrate is exposed, reaction may preferentially occur at those locations, negating the benefits of BDD. Using the methods presented in this work, it is shown that a significant increase in surface area of BDD is possible.

BDD was fabricated and characterized by SEM, Raman spectroscopy, FTIR, and CV. SEM indicated a microcrystalline morphology that is continuous and uniform. Raman spectroscopy showed the presence of a high boron doping concentration and the absence of significant non-diamond carbon content. The absence of any significant nitrogen impurities is evident based on FTIR. CV was utilized with a wide variety of redox couples, indicating the usefulness of BDD in an aqueous electrolyte, RFB setting. Peak-to-peak separation and peak ratio of these redox couples on BDD is comparable to, and in some cases better than, traditional carbon-based electrodes. The durability of BDD in terms of corrosion was demonstrated through a long-term testing comparison with fuel cell grade graphite. Fouling resistance was shown with operation in a manganese electrolyte, which 
results in a manganese oxide precipitate, by undergoing a high number of cycles before and after the surface is flushed.

The electrochemical properties of the in-house grown BDD were presented, including solvent window and diffusion coefficients, determined through CV. These values compare well with published literature, indicating the electrochemical cell utilized for testing is appropriate. The difficulties of scale-up to a full cell RFB are demonstrated through several iterations, and the implementation of, several full cell designs. As a result, it was determined that a significant increase in surface area is required to actualize scaleup. Several pathways to increased surface area were demonstrated through SEM and electrochemical testing. $\mathrm{CV}$ showed that BDD grown on quartz fiber and result in extremely high current densities compared to a 2D BDD electrode. 


\section{CHAPTER 8}

\section{FUTURE RECOMMENDATIONS}

With the implementation of BDD as an electrode in a RFB, several directions can be taken to advance current research. The most obvious is to pick up where this work left off, continue the exploration of techniques to increase surface area and, ultimately, demonstrate a full cell RFB using BDD in one or both half-cells. With this demonstration comes the ability to directly compare key characteristics of RFBs. That is coulombic, voltage, and energy efficiency, energy density, and power density. Increasing the surface area of BDD can be done using several techniques. Some techniques include some type of etching or takeaway of BDD material after growth. This has been proven to increase surface area; however, the increase does not compare well with that of traditional carbonbased electrodes, such as graphite felt.

Some methods, such as presented in this work, include growth on some type of porous substrate. This can significantly increase surface area. The primary difficulty is in promoting BDD growth penetration into the porous material. Typically, after 10s of microns, radicals resulting from plasma have difficulty penetrating, and growth slows or stops. Improved fabrication techniques or growth recipes are necessary to improve the thickness of BDD grown on porous media and/or increase the penetration depth of plasma radicals. Another promising method of increasing surface area, also shown in this work, is growth of BDD on fiber structures. Fibers, such are quartz wool, come in a variety of 
materials. The difficulty in growing on fibers tends to be the concentration of plasma on fibers that stick out from the surface. This results in poor growth uniformity. Unlike some porous structures, fibers can be grown in thin layers and stacked to negate issues of plasma penetration.

Another promising research area is the exploration of other redox couples. This work has demonstrated several redox couples exist with low overpotentials to the active species and high reversibility. This indicates many more may exist. Based on the results from ferri/ferrocyanide, there may exist a redox couple that operates better than the cerium and manganese redox couples presented in this work. Some high potential redox couples not presented in this work include a series of multielectron transfer iron redox couples and cobalt. The achievement of any one of the high potential iron redox couples would be significant as iron is extremely low-cost and, it can be coupled with a low potential iron redox couple that has previously been demonstrated in other studies to be highly efficiency and reversible.

There exists a nearly infinite amount of non-aqueous redox couples. Many of which can operate at more extreme potentials then exhibited in this study, due to the utilization of non-aqueous solvents. It may be prudent to examine some of the promising non-aqueous metallic or organic redox couples on BDD. These redox couples can have benefits rivaling those of aqueous redox couples, such as tunability, increased solubility, low rates of crossover, etc. With the inclusion of non-aqueous redox couples, research on BDD as an electrode for RFBs is nearly infinite for the foreseeable future. 


\section{REFERENCES}

[1] Almeida, E. C., Diniz, A. V., Trava-Airoldi, V. J., \& Ferreira, N. G. (2005). Electrochemical characterization of doped diamond-coated carbon fibers at different boron concentrations. Thin Solid Films, 485(1), 241-246. doi:https://doi.org/10.1016/j.tsf.2005.03.053

[2] Alotto, P., Guarnieri, M., \& Moro, F. (2014). Redox flow batteries for the storage of renewable energy: A review. Renewable and Sustainable Energy Reviews, 29, 325335. doi:https://doi.org/10.1016/j.rser.2013.08.001

[3] Amini, K., \& Pritzker, M. D. (2019). Improvement of zinc-cerium redox flow batteries using mixed methanesulfonate-chloride negative electrolyte. Applied Energy, 255. doi:10.1016/j.apenergy.2019.113894

[4] Arenas, L. F., León, C. P. d., \& Walsh, F. C. (2016). Mass transport and active area of porous $\mathrm{Pt} / \mathrm{Ti}$ electrodes for the $\mathrm{Zn}$-Ce redox flow battery determined from limiting current measurements. Electrochimica Acta, 221(Supplement C), 154-166. doi:https://doi.org/10.1016/j.electacta.2016.10.097

[5] Ashfold, M. N. R., Mahoney, E. J. D., Mushtaq, S., Truscott, B. S., \& Mankelevich, Y. A. (2017). What [plasma used for growing] diamond can shine like flame? Chemical Communications, 53(76), 10482-10495. doi:10.1039/C7CC05568D

[6] Ashimura, S., \& Miyake, Y. (1971). Denki Kagaku, 39, 977.

[7] Ashimura, S., \& Miyake, Y. (1975). Denki Kagaku, 43, 214.

[8] Ashimura, S., \& Miyake, Y. (1976). Denki Kagaku, 44, 50.

[9] Bard, A. J., \& Faulkner, L. R. (2001). Electrochemical methods : fundamentals and applications (2nd ed. ed.). New York: Wiley.

[10] Bard, A. J., Parsons, R., Jordan, J., International Union of, P., \& Applied, C. (1985). Standard potentials in aqueous solution (1st ed. ed.). New York: M. Dekker.

[11] Beaudin, M., Zareipour, H., Schellenberglabe, A., \& Rosehart, W. (2010). Energy storage for mitigating the variability of renewable electricity sources: An updated review. Energy for Sustainable Development, 14(4), 302-314. doi:https://doi.org/10.1016/j.esd.2010.09.007

[12] Brooker, R. P., Bell, C. J., Bonville, L. J., Kunz, H. R., \& Fenton, J. M. (2015). Determining Vanadium Concentrations Using the UV-Vis Response Method. Journal of The Electrochemical Society, 162(4), 608-613. doi:10.1149/2.0371504jes

[13] Chen, H., Cong, T. N., Yang, W., Tan, C., Li, Y., \& Ding, Y. (2009). Progress in electrical energy storage system: A critical review. Progress in Natural Science, 19(3), 291-312. doi:https://doi.org/10.1016/j.pnsc.2008.07.014 
[14] Chen, R., \& Hempelmann, R. (2016). Ionic liquid-mediated aqueous redox flow batteries for high voltage applications. Electrochemistry Communications, 70(Supplement C), 56-59. doi:https://doi.org/10.1016/j.elecom.2016.07.003

[15] Chen, W., Li, G., Pei, A., Li, Y., Liao, L., Wang, H., .. Cui, Y. (2018). A manganesehydrogen battery with potential for grid-scale energy storage. Nature Energy, 3(5), 428-435. doi:10.1038/s41560-018-0147-7

[16] Chevallier, J., Lusson, A., Ballutaud, D., Theys, B., Jomard, F., Deneuville, A., . . . Bustarret, E. (2001). Hydrogen-acceptor interactions in diamond. Diamond and Related Materials, 10(3), 399-404. doi:https://doi.org/10.1016/S09259635(00)00432-5

[17] Ciprios, G., Jr., W. E., \& Grimes, P. G. (1977). NASA contract Rep. No. NAS3-135$206,1 \& 2$.

[18] Cobb, S. J., Ayres, Z. J., \& Macpherson, J. V. (2018). Boron Doped Diamond: A Designer Electrode Material for the Twenty-First Century. Annual Review of Analytical Chemistry, 11(1), 463-484. doi:10.1146/annurev-anchem-061417010107

[19] Council, W. E. (2016). E-storage: Shifting from cost to value

Wind and solar applications In. London: World Energy Council.

[20] Crawford, A., Viswanathan, V., Stephenson, D., Wang, W., Thomsen, E., Reed, D., . . . Sprenkle, V. (2015). Comparative analysis for various redox flow batteries chemistries using a cost performance model. Journal of Power Sources, 293, 388399. doi:https://doi.org/10.1016/j.jpowsour.2015.05.066

[21] Darling, R. M., Gallagher, K. G., Kowalski, J. A., Ha, S., \& Brushett, F. R. (2014). Pathways to low-cost electrochemical energy storage: a comparison of aqueous and nonaqueous flow batteries. Energy \& Environmental Science, 7(11), 3459-3477. doi:10.1039/C4EE02158D

[22] Dong-Jun, P., Kwang-Sun, J., Cheol-Hwi, R., \& Gab-Jin, H. (2017). Performance of the all-vanadium redox flow battery stack. Journal of Industrial and Engineering Chemistry, 45, 387-390. doi:10.1016/j.jiec.2016.10.007

[23] Dong, Y.-R., Kaku, H., Hanafusa, K., Moriuchi, K., \& Shigematsu, T. (2015). A Novel Titanium/Manganese Redox Flow Battery. ECS Transactions, 69(18), 59-67. doi:10.1149/06918.0059ecst

[24] Dunn, B., Kamath, H., \& Tarascon, J.-M. (2011). Electrical Energy Storage for the Grid: A Battery of Choices. Science, 334(6058), 928-935. doi:10.1126/science.1212741

[25] EnergyAustralia. (2018). Clean energy fact sheet. In. energyaustralia.com.au.

[26] Fang, C., Jia, X., Chen, N., Li, Y., Guo, L., Chen, L., . . Liu, X. (2016). HPHT synthesis of $\mathrm{N}-\mathrm{H}$ co-doped diamond single crystals. Journal of Crystal Growth, 436, 34-39. doi:https://doi.org/10.1016/j.jcrysgro.2015.11.042

[27] Fang, C., Zhang, Y., Zhang, Z., Shan, C., Shen, W., \& Jia, X. (2018). Preparation of "natural" diamonds by HPHT annealing of synthetic diamonds. CrystEngComm, 20(4), 505-511. doi:10.1039/C7CE02013A

[28] Ferrer, N., \& Nogués-Carulla, J. M. (1996). Characterisation study of cut gem diamond by IR spectroscopy. Diamond and Related Materials, 5(6), 598-602. doi:https://doi.org/10.1016/0925-9635(95)00479-3 
[29] Frey, P. A., \& Reed, G. H. (2012). The Ubiquity of Iron. ACS Chemical Biology, 7(9), 1477-1481. doi:10.1021/cb300323q

[30] Geis, M. W., Wade, T. C., Wuorio, C. H., Fedynyshyn, T. H., Duncan, B., Plaut, M. E., . . Hollis, M. A. (2018). Progress Toward Diamond Power Field-Effect Transistors. physica status solidi (a), 215(22), 1800681. doi:10.1002/pssa.201800681

[31] Giner, J., Swette, L., \& Cahill, K. (1976). NASA Contract NAS3-19760, NASA CR134705.

[32] Glesener, J. W., Natishan, P. M., O'Grady, W. E., Aiken, J., \& Morrish, A. A. (1998). Fabrication of a high surface area boron-doped diamond coated tungsten mesh for electrochemical applications. Materials Letters, 37(3), 138-142. doi:https://doi.org/10.1016/S0167-577X(98)00083-4

[33] Gnamba, C. Q.-M., Appia, F. T. r. A., Loba, E. M. H. 1. n., Sanogo, I., \& Ouattara, L. (2015). Electrochemical oxidation of amoxicillin in its pharmaceutical formulation at boron doped diamond (BDD) electrode. Journal of Electrochemical Science and Engineering, 5(2). doi:10.5599/jese. 186

[34] Gong, K., Xu, F., Lehrich, M. G., Ma, X., Gu, S., \& Yan, Y. (2017). Exploiting Immiscible Aqueous-Nonaqueous Electrolyte Interface toward a Membraneless Redox-Flow Battery Concept. Journal of The Electrochemical Society, 164(12), A2590-A2593. doi:10.1149/2.1241712jes

[35] González, Z., Flox, C., Blanco, C., Granda, M., Morante, J. R., Menéndez, R., \& Santamaría, R. (2017). Outstanding electrochemical performance of a graphenemodified graphite felt for vanadium redox flow battery application. Journal of Power Sources, 338, 155-162. doi:https://doi.org/10.1016/j.jpowsour.2016.10.069

[36] Gubler, L. (2019). Membranes and separators for redox flow batteries. Current Opinion in Electrochemistry, 18, 31-36. doi:https://doi.org/10.1016/j.coelec.2019.08.007

[37] Gür, T. M. (2018). Review of electrical energy storage technologies, materials and systems: challenges and prospects for large-scale grid storage. Energy \& Environmental Science, 11(10), 2696-2767. doi:10.1039/C8EE01419A

[38] Gyuk, I., Johnson, M., Vetrano, J., Lynn, K., Parks, W., Handa, R., . . Braccio, R. (2013). Grid Energy Storage. Retrieved from

[39] Hannan, M. A., Hoque, M. M., Mohamed, A., \& Ayob, A. (2017). Review of energy storage systems for electric vehicle applications: Issues and challenges. Renewable and Sustainable Energy Reviews, 69, 771-789. doi:https://doi.org/10.1016/j.rser.2016.11.171

[40] He, Y., Lin, H., Guo, Z., Zhang, W., Li, H., \& Huang, W. (2019). Recent developments and advances in boron-doped diamond electrodes for electrochemical oxidation of organic pollutants. Separation and Purification Technology, 212, 802-821. doi:https://doi.org/10.1016/j.seppur.2018.11.056

[41] Hong, T., \& Xue, F. (2009, 27-31 March 2009). Investigation on Manganese $\left(\mathrm{Mn}^{2}+/ \mathrm{Mn}^{3}+\right)$ - Vanadium $\left(\mathrm{V}^{2}+\mathrm{V}^{3}+\right)$ Redox Flow Battery. Paper presented at the 2009 Asia-Pacific Power and Energy Engineering Conference.

[42] Howitt, M. (2020). Long duration CAES and the affordable energy transition.

[43] Hu, M., Bi, N., Li, S., Su, T., Hu, Q., Ma, H., \& Jia, X. (2017). Synthesis and characterization of boron and nitrogen co-doped diamond crystals under high 
pressure and high temperature conditions. CrystEngComm, 19(31), 4571-4575. doi:10.1039/C7CE00709D

[44] Huang, G., Zheng, Y., Peng, L., Li, Z., Jia, X., \& Ma, H. (2015). Crystallization of HPHT diamond crystals in a floatage system under the influence of nitrogen and hydrogen simultaneously. CrystEngComm, 17(34), 6504-6511. doi:10.1039/C5CE01225B

[45] Huang, Y., Gu, S., Yan, Y., \& Li, S. F. Y. (2015). Nonaqueous redox-flow batteries: features, challenges, and prospects. Current Opinion in Chemical Engineering, 8, 105-113. doi:https://doi.org/10.1016/j.coche.2015.04.001

[46] Hutton, L. A., Iacobini, J. G., Bitziou, E., Channon, R. B., Newton, M. E., \& Macpherson, J. V. (2013). Examination of the Factors Affecting the Electrochemical Performance of Oxygen-Terminated Polycrystalline Boron-Doped Diamond Electrodes. Analytical Chemistry, 85(15), 7230-7240. doi:10.1021/ac401042t

[47] Jae-Hun, K., Jin-Hyun, L., Maurya, S., Sung-Hee, S., Jae-Young, L., In Seop, C., \& Seung-Hyeon, M. (2016). Proof-of-concept experiments of an acid-base junction flow battery by reverse bipolar electrodialysis for an energy conversion system. Electrochemistry Communications, $\quad 72, \quad 157-161$. doi:10.1016/j.elecom.2016.09.025

[48] Jayathilake, B. S., Plichta, E. J., Hendrickson, M. A., \& Narayanan, S. R. (2018). Improvements to the Coulombic Efficiency of the Iron Electrode for an All-Iron Redox-Flow Battery. Journal of The Electrochemical Society, 165(9), A1630A1638. doi:10.1149/2.0451809jes

[49] Jevtić, S., Stefanović, A., Stanković, D. M., Pergal, M. V., Ivanović, A. T., Jokić, A., \& Petković, B. B. (2018). Boron-doped diamond electrode - A prestigious unmodified carbon electrode for simple and fast determination of bentazone in river water samples. Diamond and Related Materials, 81, 133-137. doi:https://doi.org/10.1016/j.diamond.2017.12.009

[50] Jing, Z., Gaopeng, J., Pan, X., Kashkooli, A. G., Mousavi, M., Aiping, Y., \& Zhongwei, C. (2018). An all-aqueous redox flow battery with unprecedented energy density. Energy \&amp; Environmental Science, 11(8), 2010-2015. doi:10.1039/c8ee00686e

[51] Kaku, H., Dong, Y.-R., Hanafusa, K., Moriuchi, K., \& Shigematsu, T. (2016). Effect of Ti(IV) Ion on Mn(III) Stability in Ti/Mn Electrolyte for Redox Flow Battery. ECS Transactions, 72(10), 1-9. doi:10.1149/07210.0001ecst

[52] Kangro, W. (1954). Germany Patent No. Deutsches Patentamt.

[53] Laboratories, S. N. (2018). DOE Global Energy Storage Database. from Sandia Coorporation

[54] Lambert, F. (2018, 9/24/2018). Tesla's massive Powerpack battery in Australia cost $\$ 66$ million and already made up to $\sim \$ 17$ million. Retrieved from https://electrek.co/2018/09/24/tesla-powerpack-battery-australia-cost-revenue/

[55] Leung, P. K., Ponce-de-León, C., Low, C. T. J., Shah, A. A., \& Walsh, F. C. (2011). Characterization of a zinc-cerium flow battery. Journal of Power Sources, 196(11), 5174-5185. doi:https://doi.org/10.1016/j.jpowsour.2011.01.095

[56] Li, Y., Sniekers, J., Malaquias, J., Li, X., Schaltin, S., Stappers, L., . . V Vankelecom, I. F. J. (2017). A non-aqueous all-copper redox flow battery with highly soluble 
active species. Electrochimica Acta, 236, 116-121. doi:https://doi.org/10.1016/i.electacta.2017.03.039

[57] Li, Z., Weng, G., Zou, Q., Cong, G., \& Lu, Y.-C. (2016). A high-energy and low-cost polysulfide/iodide redox flow battery. Nano Energy, 30(Supplement C), 283-292. doi:https://doi.org/10.1016/j.nanoen.2016.09.043

[58] Lips, S., \& Waldvogel, S. R. (2019). Use of Boron-Doped Diamond Electrodes in Electro-Organic Synthesis. ChemElectroChem, 6(6), 1649-1660. doi:10.1002/celc. 201801620

[59] Liu, C., Neale, Z. G., \& Cao, G. (2016). Understanding electrochemical potentials of cathode materials in rechargeable batteries. Materials Today, 19(2), 109-123. doi:https://doi.org/10.1016/j.mattod.2015.10.009

[60] Luo, X., Wang, J., Dooner, M., \& Clarke, J. (2015). Overview of current development in electrical energy storage technologies and the application potential in power system operation. Applied Energy, 137, 511-536. doi:https://doi.org/10.1016/j.apenergy.2014.09.081

[61] Macpherson, J. V. (2015). A practical guide to using boron doped diamond in electrochemical research. Physical Chemistry Chemical Physics, 17(5), 2935-2949. doi:10.1039/C4CP04022H

[62] May, G. J., Davidson, A., \& Monahov, B. (2018). Lead batteries for utility energy storage: A review. Journal of Energy Storage, 15, 145-157. doi:https://doi.org/10.1016/j.est.2017.11.008

[63] Mongird, K., Viswanathan, V., Balducci, P., Alam, J., Fotedar, V., Koritarov, V., \& Hadjerioua, B. (2019). Energy Storage Technology and Cost Characterization Report. Retrieved from

[64] Na, Z., Sun, X., \& Wang, L. (2018). Surface-functionalized graphite felts: Enhanced performance in cerium-based redox flow batteries. Carbon, 138, 363-368. doi:https://doi.org/10.1016/j.carbon.2018.07.052

[65] Na, Z., Xu, S., Yin, D., \& Wang, L. (2015). A cerium-lead redox flow battery system employing supporting electrolyte of methanesulfonic acid. Journal of Power Sources, 295(Supplement $\quad$ C), 28-32. doi:https://doi.org/10.1016/j.jpowsour.2015.06.115

[66] Narins, T. P. (2017). The battery business: Lithium availability and the growth of the global electric car industry. The Extractive Industries and Society, 4(2), 321-328. doi:https://doi.org/10.1016/j.exis.2017.01.013

[67] Nebel, C. E., Rezek, B., Shin, D., Uetsuka, H., \& Yang, N. (2007). Diamond for biosensor applications. Journal of Physics D: Applied Physics, 40(20), 6443-6466. doi:10.1088/0022-3727/40/20/s21

[68] Nikiforidis, G., Berlouis, L., Hall, D., \& Hodgson, D. (2013). Impact of electrolyte composition on the performance of the zinc-cerium redox flow battery system. Journal of Power Sources, 243, 691-698. doi:https://doi.org/10.1016/j.jpowsour.2013.06.045

[69] Nikiforidis, G., Berlouis, L., Hall, D., \& Hodgson, D. (2014). Charge/discharge cycles on Pt and Pt-Ir based electrodes for the positive side of the Zinc-Cerium hybrid redox flow battery. Electrochimica Acta, 125, 176-182. doi:https://doi.org/10.1016/j.electacta.2014.01.075 
[70] Notsu, H., Fukazawa, T., Tatsuma, T., Tryk, D. A., \& Fujishima, A. (2001). Hydroxyl Groups on Boron-Doped Diamond Electrodes and Their Modification with a Silane Coupling Agent. Electrochemical and Solid-State Letters, 4(3), H1-H3. doi:10.1149/1.1346556

[71] O'Donnell, P. M., Gahn, R. F., \& Pfeiffer, W. (1976). Paper presented at the Twelfth Photovoltaic Specialists Conf., Baton Rouge, Louisiana.

[72] Pan, H., Shao, Y., Yan, P., Cheng, Y., Han, K. S., Nie, Z., . . Liu, J. (2016). Reversible aqueous zinc/manganese oxide energy storage from conversion reactions. Nature Energy, 1, 16039. doi:10.1038/nenergy.2016.39

https://www.nature.com/articles/nenergy201639\#supplementary-information

[73] Panizza, M. (2018). Boron-Doped Diamond Electrodes. In K. Wandelt (Ed.), Encyclopedia of Interfacial Chemistry (pp. 393-400). Oxford: Elsevier.

[74] Peng, X., Chu, J., Wang, L., Duan, S., \& Feng, P. (2017). Boron-doped diamond nanowires for CO gas sensing application. Sensors and Actuators B: Chemical, 241, 383-389. doi:https://doi.org/10.1016/j.snb.2016.10.009

[75] Petek, T. J., Hoyt, N. C., Savinell, R. F., \& Wainright, J. S. (2015). Slurry electrodes for iron plating in an all-iron flow battery. Journal of Power Sources, 294(Supplement C), 620-626. doi:https://doi.org/10.1016/j.jpowsour.2015.06.050

[76] Petek, T. J., Hoyt, N. C., Savinell, R. F., \& Wainright, J. S. (2015). Slurry electrodes for iron plating in an all-iron flow battery. Journal of Power Sources, 294, 620-626. doi:https://doi.org/10.1016/j.jpowsour.2015.06.050

[77] Pissoort, P. A. (1933). France Patent No.: M. D. C. E. D. L'INDUSTRIE.

[78] Posner, A. (1955). Redox fuel cell. Fuel, 34(3), 330-338.

[79] Rastler, D. (2010). Electricity Energy Storage Technology Options: A White Paper Primer on Applications, Costs, and Benefits In. Palo Alto, CA: EPRI.

[80] Reynard, D., Dennison, C. R., Battistel, A., \& Girault, H. H. (2018). Efficiency improvement of an all-vanadium redox flow battery by harvesting low-grade heat. Journal of Power Sources, 390, 37. doi:https://doi.org/10.1016/j.jpowsour.2018.03.074

[81] Rosewater, D., \& Williams, A. (2015). Analyzing system safety in lithium-ion grid energy storage. Journal of Power Sources, 300, 460-471. doi:https://doi.org/10.1016/j.jpowsour.2015.09.068

[82] Roy, N., Shibano, Y., Terashima, C., Katsumata, K. I., Nakata, K., Kondo, T., . . . Fujishima, A. (2016). Ionic-liquid-assisted selective and controlled electrochemical $\mathrm{CO} 2$ reduction at $\mathrm{Cu}$-modified boron-doped diamond electrode. ChemElectroChem, 3(7), 1044-1047. doi:10.1002/celc.201600105

[83] Sanz, L., Lloyd, D., Magdalena, E., Palma, J., Anderson, M., \& Kontturi, K. (2015). Study and characterization of positive electrolytes for application in the aqueous all-copper redox flow battery. Journal of Power Sources, 278, 175-182. doi:https://doi.org/10.1016/j.jpowsour.2014.12.034

[84] Sanz, L., Lloyd, D., Magdalena, E., Palma, J., \& Kontturi, K. (2014). Description and performance of a novel aqueous all-copper redox flow battery. Journal of Power Sources, 268, 121-128. doi:https://doi.org/10.1016/j.jpowsour.2014.06.008

[85] Shi, C., Li, C., Li, M., Li, H., Dai, W., Wu, Y., \& Yang, B. (2016). Fabrication of porous boron-doped diamond electrodes by catalytic etching under hydrogen- 
argon plasma. Applied Surface Science, 360, 315-322. doi:https://doi.org/10.1016/j.apsusc.2015.11.028

[86] Silva, L. L. G., Ferreira, N. G., \& Corat, E. J. (2008). Morphological and electrochemical properties of boron-doped diamond films on carbon cloths with enhanced surface area. Thin Solid Films, 516(15), 4934-4939. doi:https://doi.org/10.1016/j.tsf.2007.09.035

[87] Siuzdak, K., Niedziałkowski, P., Sobaszek, M., Łęga, T., Sawczak, M., Czaczyk, E., .. . Bogdanowicz, R. (2019). Biomolecular influenza virus detection based on the electrochemical impedance spectroscopy using the nanocrystalline boron-doped diamond electrodes with covalently bound antibodies. Sensors and Actuators B: Chemical, 280, 263-271. doi:https://doi.org/10.1016/j.snb.2018.10.005

[88] Skyllas-Kazacos, M., Rychcik, M., Robins, R. G., Fane, A. G., \& Green, M. A. (1986). New All-Vanadium Redox Flow Cell. Journal of The Electrochemical Society, 133(5), 1057-1058. doi:10.1149/1.2108706

[89] Sleightholme, A. E. S., Shinkle, A. A., Liu, Q., Li, Y., Monroe, C. W., \& Thompson, L. T. (2011). Non-aqueous manganese acetylacetonate electrolyte for redox flow batteries. Journal of Power Sources, 196(13), 5742-5745. doi:https://doi.org/10.1016/j.jpowsour.2011.02.020

[90] Soloveichik, G. L. (2015). Flow Batteries: Current Status and Trends. Chemical Reviews, 115(20), 11533-11558. doi:10.1021/cr500720t

[91] Suo, N., Huang, H., Wu, A., Cao, G., Hou, X., \& Zhang, G. (2018). Porous boron doped diamonds as metal-free catalysts for the oxygen reduction reaction in alkaline solution. Applied Surface Science, 439, 329-335. doi:https://doi.org/10.1016/j.apsusc.2017.12.198

[92] Suresh, S., Ulaganathan, M., Venkatesan, N., Periasamy, P., \& Ragupathy, P. (2018). High performance zinc-bromine redox flow batteries: Role of various carbon felts and cell configurations. Journal of Energy Storage, 20, 134-139. doi:https://doi.org/10.1016/j.est.2018.09.006

[93] Thaller, L. H. (1974). Paper presented at the Ninth Intersoc. Energy Conv. Eng. Conf., San Francisco, CA.

[94] Ullah, M., Ahmed, E., Welch, K., Majdi, S., Khalid, N. R., \& Ahmad, M. (2013). Growth of Nitrogen-Incorporated Diamond Films Using Hot-Filament Chemical Vapor Deposition Technique (Vol. 19).

[95] van Egmond, W. J., Saakes, M., Porada, S., Meuwissen, T., Buisman, C. J. N., \& Hamelers, H. V. M. (2016). The concentration gradient flow battery as electricity storage system: Technology potential and energy dissipation. Journal of Power Sources, 325, 129-139. doi:https://doi.org/10.1016/j.jpowsour.2016.05.130

[96] Vanýsek, P. (1996). Modern techniques in electroanalysis. New York: Wiley.

[97] Vertes, M. A., Katsoulis, E. G., Oxley, J. E., \& Alfredson, K. (1966). Zinc/air high energy density rechargeable energy storage system. Ft. Belvoir: Defense Technical Information Center.

[98] Wang, Q., \& Daoud, W. A. (2019). Reaction kinetics of cerium on glassy carbon with in situ electrochemical treatment for cerium-based flow battery. Electrochimica Acta, 315, 1-8. doi:https://doi.org/10.1016/j.electacta.2019.05.083

[99] Watanabe, T., Shibano, S., Maeda, H., Sugitani, A., Katayama, M., Matsumoto, Y., \& Einaga, Y. (2016). Fabrication of a Microfluidic Device with Boron-doped 
Diamond Electrodes for Electrochemical Analysis. Electrochimica Acta, 197, 159166. doi:https://doi.org/10.1016/j.electacta.2015.11.035

[100] Wei, L., Wu, M. C., Zhao, T. S., Zeng, Y. K., \& Ren, Y. X. (2018). An aqueous alkaline battery consisting of inexpensive all-iron redox chemistries for large-scale energy storage. Applied Energy, 215, 98-105. doi:https://doi.org/10.1016/j.apenergy.2018.01.080

[101] Wei, L., Zhao, T. S., Zeng, L., Zhou, X. L., \& Zeng, Y. K. (2016). Copper nanoparticle-deposited graphite felt electrodes for all vanadium redox flow batteries. Applied Energy, 180, 386-391. doi:https://doi.org/10.1016/j.apenergy.2016.07.134

[102] Wesoff, E. (2011). Exploding Sodium Sulfur Batteries From NGK Energy Storage. Greenech Media.

[103] Winsberg, J., Hagemann, T., Janoschka, T., Hager, M. D., \& Schubert, U. S. (2017). Redox-Flow Batteries: From Metals to Organic Redox-Active Materials. Angewandte Chemie International Edition, 56(3), 686-711. doi:10.1002/anie.201604925

[104] Wu, M. C., Zhao, T. S., Jiang, H. R., Zeng, Y. K., \& Ren, Y. X. (2017). Highperformance zinc bromine flow battery via improved design of electrolyte and electrode. Journal of Power Sources, 355, 62-68. doi:https://doi.org/10.1016/j.jpowsour.2017.04.058

[105] Xie, Z., Zhou, D., Xiong, F., Zhang, S., \& Huang, K. (2011). Cerium-zinc redox flow battery: Positive half-cell electrolyte studies. Journal of Rare Earths, 29(6), 567573. doi:https://doi.org/10.1016/S1002-0721(10)60499-1

[106] Xing, X., Liu, Q., Wang, B., Lemmon, J. P., \& Xu, W. Q. (2020). A low potential solvent-miscible 3-methylbenzophenone anolyte material for high voltage and energy density all-organic flow battery. Journal of Power Sources, 445, 227330. doi:https://doi.org/10.1016/j.jpowsour.2019.227330

[107] Xu, J., Natsui, K., Naoi, S., Nakata, K., \& Einaga, Y. (2018). Effect of doping level on the electrochemical reduction of $\mathrm{CO} 2$ on boron-doped diamond electrodes. Diamond and Related Materials, 86, 167-172. doi:https://doi.org/10.1016/j.diamond.2018.04.028

[108] Xu, Q., Ji, Y. N., Qin, L. Y., Leung, P. K., Qiao, F., Li, Y. S., \& Su, H. N. (2018). Evaluation of redox flow batteries goes beyond round-trip efficiency: A technical review. Journal of Energy Storage, 16, 108-115. doi:https://doi.org/10.1016/j.est.2018.01.005

[109] Xue, F.-Q., Wang, Y.-L., Wang, W.-H., \& Wang, X.-D. (2008). Investigation on the electrode process of the $\mathrm{Mn}(\mathrm{II}) / \mathrm{Mn}(\mathrm{III})$ couple in redox flow battery. Electrochimica Acta, 53(22), 6636-6642. doi:https://doi.org/10.1016/j.electacta.2008.04.040

[110] Yang, B., Hoober-Burkhardt, L., Krishnamoorthy, S., Murali, A., Prakash, G. K. S., \& Narayanan, S. R. (2016). High-Performance Aqueous Organic Flow Battery with Quinone-Based Redox Couples at Both Electrodes. Journal of The Electrochemical Society, 163(7), A1442-A1449. doi:10.1149/2.1371607jes

[111] Yang, N., Foord, J. S., \& Jiang, X. (2016). Diamond electrochemistry at the $\begin{array}{lllll}\text { nanoscale: } & \text { A review. }\end{array}$ doi:https://doi.org/10.1016/j.carbon.2015.11.061 
[112] Yang, Z. G. (2017). It's Big and Long-Lived, and It Won't Catch Fire: The Vanadium Redox-Flow Battery. Retrieved from https://spectrum.ieee.org/green-tech/fuelcells/its-big-and-longlived-and-it-wont-catch-fire-the-vanadium-redoxflowbattery

[113] Yu, D., Yu, Z., Yutao, L., Goodenough, J. B., \& Guihua, Y. (2017). A highperformance all-metallocene-based, non-aqueous redox flow battery. Energy \&amp; Environmental Science, 10(2), 491-497. doi:10.1039/c6ee02057g

[114] Zhang, J., Jiang, G., Xu, P., Ghorbani Kashkooli, A., Mousavi, M., Yu, A., \& Chen, Z. (2018). An all-aqueous redox flow battery with unprecedented energy density. Energy and Environmental Science, 11(8), 2010-2015. doi:10.1039/c8ee00686e

[115] Zhen, Y., Zhang, C., Yuan, J., Zhao, Y., \& Li, Y. (2020). A high-performance alliron non-aqueous redox flow battery. Journal of Power Sources, 445, 227331. doi:https://doi.org/10.1016/j.jpowsour.2019.227331

[116] Zhou, X., Lin, L., Lv, Y., Zhang, X., \& Wu, Q. (2018). A Sn-Fe flow battery with excellent rate and cycle performance. Journal of Power Sources, 404, 89-95. doi:https://doi.org/10.1016/j.jpowsour.2018.10.011 


\section{APPENDIX}

The colors corresponding with the different oxidation states of vanadium are shown in Figure A1. This solution consisted of $\mathrm{V}_{2} \mathrm{O}_{5}$ and DI water with glycerol as an oxidant. The amount of glycerol can be controlled to control the number of ions reduced. With that, the overall oxidation state of the vanadium ions in solution can be controlled. The solution was continuously stirred with a magnetic stirrer and a nitrogen blanket was used so that the solution did not oxidize with the air. Yellow corresponds with $\mathrm{VO}_{2}{ }^{+}$, blue with $\mathrm{VO}^{2+}$, green with $\mathrm{V}^{3+}$, and purple with $\mathrm{V}^{2+}$.

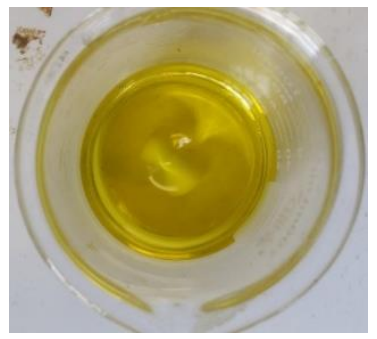

(a)

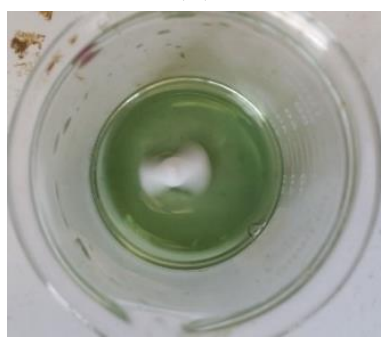

(c)

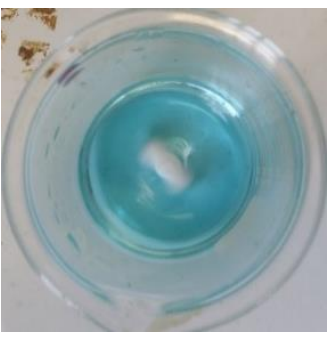

(b)

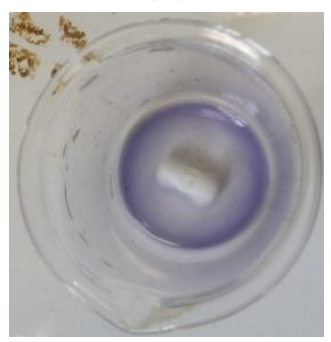

(d)

Figure A1. Reduction of $\mathrm{V}_{2} \mathrm{O}_{5}$ in $\mathrm{H}_{2} \mathrm{SO}_{4}$ using glycerol as a reducing agent to exhibit the four oxidation states of vanadium (a) $\mathrm{VO}_{2}{ }^{+}$, (b) $\mathrm{VO}^{2+}$, (c) $\mathrm{V}^{3+}$, and (d) $\mathrm{V}^{2+}$. 
The initial electrochemical cell setup is shown in Figure A2. An electrochemical cell designed for fuel cells was used. The tubes in with the electrolyte flows is transparent so the oxidation state can be qualitatively observed. The beginning solution is shown in Figure $\mathrm{A} 2(\mathrm{a})$, that is $\mathrm{VO}^{2+}$ in both half-cells. $\mathrm{VO}^{2+}$ must be reduced twice electrochemically to achieve $\mathrm{V}^{2+}$, purple, which is observed in Figure A2(b and c). Figure A2(d) shows a green color (combination of yellow, $\mathrm{VO}_{2}{ }^{+}$, and green, $\mathrm{VO}^{2+}$ ) which is transition to yellow while the other electrolyte is transitioning from blue to purple.

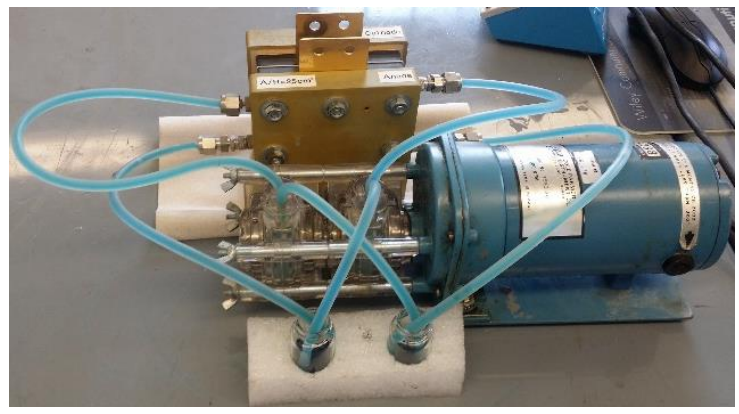

(a)

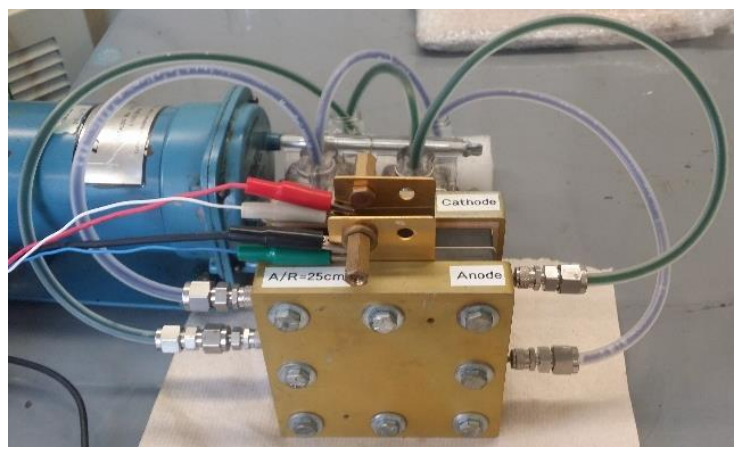

(c)

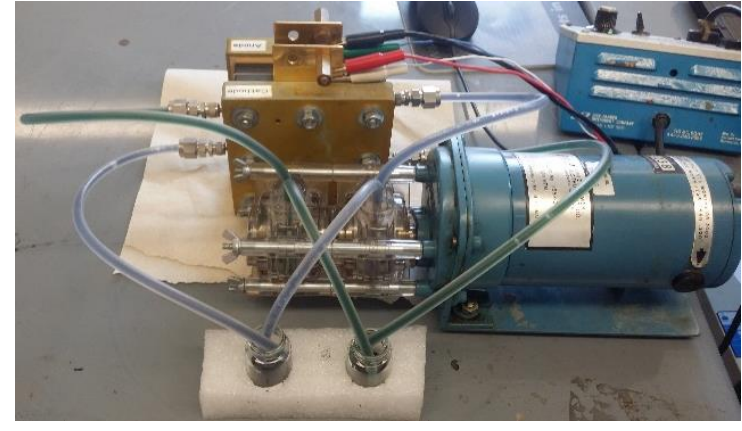

(b)

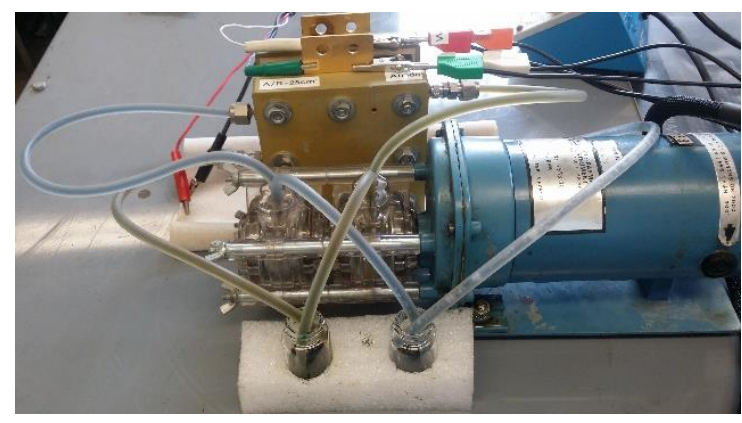

(d)

Figure A2. Electrochemical reduction of vanadium starting with $\mathrm{V}_{2} \mathrm{O}_{5}$ in a sulfuric acid solution partially reduced to (a) $\mathrm{VO}^{2+}$, using a measured amount of glycerol, in both half cells, (b and c) $\mathrm{V}^{2+}$ and mixed $\mathrm{VO}_{2}{ }^{+}$and $\mathrm{VO}^{2+}$, and (d) $\mathrm{VO}_{2}{ }^{+}$and mixed $\mathrm{V}^{3}$ and $\mathrm{V}^{2+}$. 
At the beginning, alternative redox couples were explored using graphite rods. A titanium redox couple, $\mathrm{TiO}_{2}{ }^{+} / \mathrm{Ti}^{3+}$, was tested, shown in Figure A3(a). This redox couple showed good reversibility, low overpotential, and high kinetics on a graphite rod. An iodine redox couple $\mathrm{I}_{3}^{-} / \mathrm{I}^{-}$was also tested, Figure $\mathrm{A} 3(\mathrm{~b})$. This redox couple shows good reversibility but, high overpotential and lower kinetics compared to the titanium redox couple.

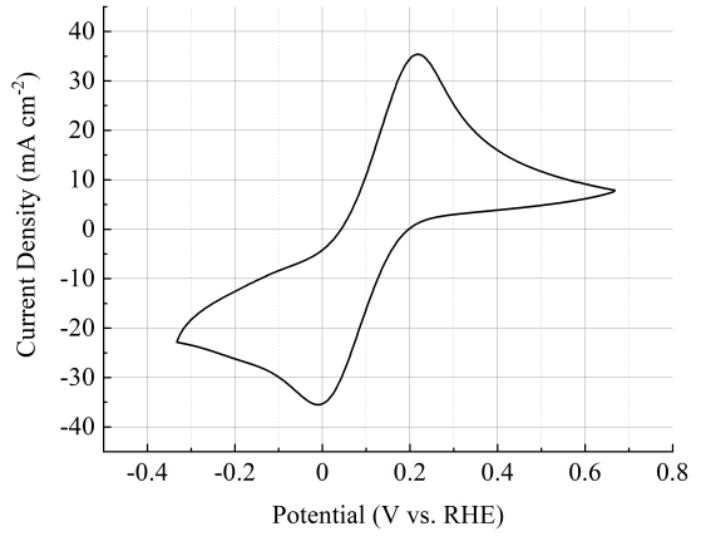

(a)

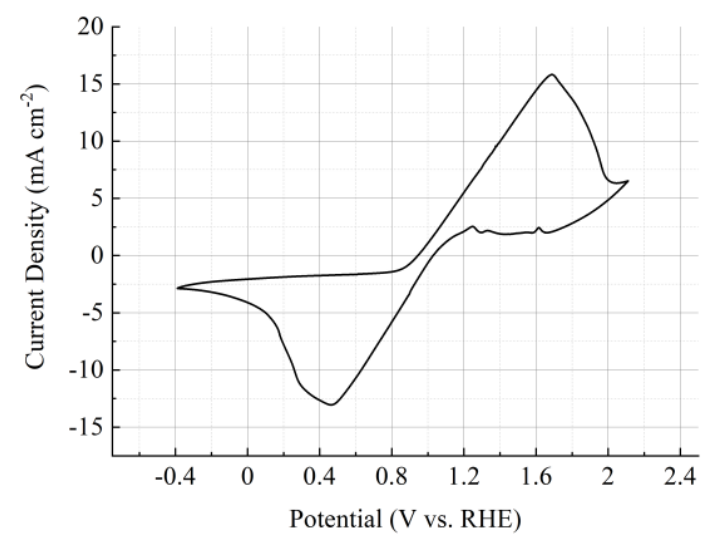

(b)

Figure A3. Examination of alternative redox couples on a graphite rod using CV at 20 $\mathrm{mV} \mathrm{s}^{-1}$ (a) $1 \mathrm{M} \mathrm{TiO}\left(\mathrm{SO}_{4}\right)$ in $3 \mathrm{M} \mathrm{H}_{2} \mathrm{SO}_{4}$ and (b) $100 \mathrm{mM} \mathrm{KI}$ without a supporting electrolyte.

The three-electrode setup for testing graphite felt is shown in Figure A4. This electrochemical cell consists of a beaker and a rubber stopper with holes for electrodes. The graphite felt was connected to the potentiostat by running a platinum wire through the middle of the structure. The platinum wire was fitted to a plastic cylinder with connected, embedded electric connector on which the alligator clips of the potentiostat clip. The 
counter electrode was also graphite felt in Figure A4 and, the reference electrode was $\mathrm{Ag} / \mathrm{AgCl}$ in saturated $\mathrm{KCl}$.

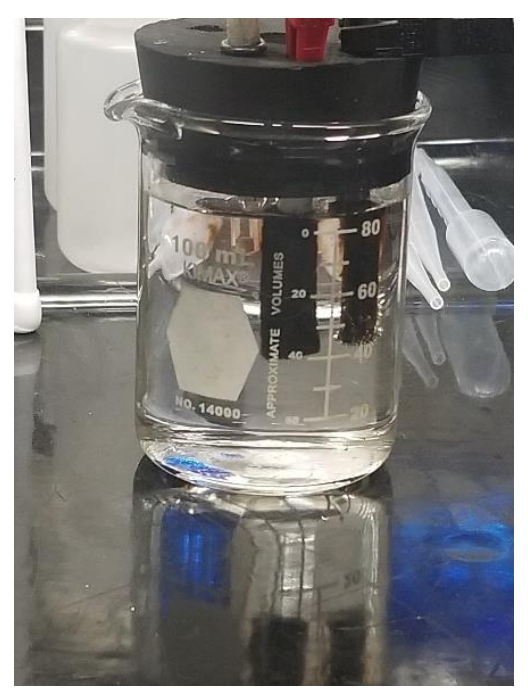

Figure A4. Three-electrode setup using a beaker, rubber stopper, graphite felt working and counter electrode, and $\mathrm{Ag} / \mathrm{AgCl}$ reference electrode.

BDD layers were grown using microwave plasma-assisted chemical vapor deposition in the "small" Seki reactor ("small" refers to the power limit which is less than the "big" Seki reactor). The following steps were taken during fabrication:

1. Substrates were prepared into roughly $1 \mathrm{~cm}$ squares. Metal substrates, tungsten, and molybdenum were cut with either scissor or snips, with a focus on maintaining a flat structure. Silicon substrates were broken into $1 \mathrm{~cm}$ squares by utilizing a diamond scribe to initiate a crack that then propagates along a crystal plane ( silicon conveniently has crystal planes at a 90 degree angle which can be taken advantage off to form squares). 
2. The metal substates were sanded with 400 grit sandpaper to remove any high points, improving the flatness, and provided shallow crevices for nano-diamond seed powder to reside. Silicon substrates were not sanded.

3. The substrates were cleaned with methanol.

4. Nano-diamond powder, which acts as a seed for diamond growth, was applied to the substrates. In all instances, a cotton swab was dipped into a solution of nanodiamond powder and methanol, then forcefully rubbed against the surface of the substrate. This creates additional scratches on the surface while deposited the nano-diamond seeds. In some instances, primarily with silicon substrates, two substrates, with growth surfaces facing each other, and after applying the nanodiamond powder, were rubbed together.

5. The substrates were placed in the container with methanol and nano-diamond powder and ultrasonicated for 1 hour. The substrates were placed with the growth surface facing up.

6. The substrates were rinsed with methanol.

7. The substrates were ultrasonicated for 1 hour in fresh methanol.

8. The substrates were dried and then placed on the growth stage (a graphite or molybdenum disk)

9. The growth stage was placed into the reactor and the reactor closed. Before closing, the gasket and mating surface were wiped clean with methanol to provide a good seal.

10. A vacuum was applied to the reaction chamber using the small valve.

11. Once below 20 torr, the large valve was opened. 
12. After reaching full vacuum, both valves were closed to ensure there is no leak in the reaction chamber.

13. Gases are turned on and the system is purged 3 times. The flow rates are dependent on the desired recipe. A standard for starting flow rates in this reactor is $250 \mathrm{sccm} \mathrm{H}_{2}, 10 \mathrm{sccm} \mathrm{CH}_{4}$, and $0.2 \mathrm{~B}_{2} \mathrm{H}_{6}$. Purging is done by close the vacuum valves, allowing the chamber to reach $\sim 100$ torr, and then vacuum is again applied. The purpose of purging is to remove a significant portion of contaminant gas particles from the reaction chamber.

14. The chamber is increased to the pressure at which the plasma will be initiated (typically 24 torr).

15. The power of the microwave source is set to $300 \mathrm{~W}$. While adjusting the reflected watts to zero. This step requires training from an experienced user.

16. Once the system stabilizes, the main vacuum valve is briefly opened to initiate the plasma.

17. The reflected watts are again adjusted to zero while the plasma position and size is checked. Any irregularity should be followed by shutdown of the reaction (power off and vacuum applied).

18. The power, pressure, and gas flow rates were adjusted slowly, while maintaining close to zero reflected watts, until the desired recipe is achieved. A standard recipe used is $300 \mathrm{sccm} \mathrm{H}_{2}, 5 \mathrm{sccm} \mathrm{CH} 4,0.2 \mathrm{sccm} \mathrm{B} \mathrm{B}_{6}, 40$ torr, and $1200 \mathrm{~W}$.

19. After the desired growth time (typically around 24 hours), the reactor is shutdown. Shutdown procedures can vary. In general, all gases other the $\mathrm{H}_{2}$ are stopped and the microwave power shutoff followed by application of a vacuum. 
The substrate was then allowed to cool under hydrogen flow for a minimum of 1 hour.

20. The vacuum valves are closed, and the chamber is slowly vented to atmosphere pressure.

21. The reaction chamber is opened and the growth stage with samples are removed. The first series of BDD samples were tested in the same configuration shown in Figure A4. To do this, the samples were encased in epoxy and affixed to a glass tube inside which a wire connects to the external connections. The wire is connected to the back of the sample and covered in epoxy. Figure A5(a) shows a platinum foil prepared in this way. The surface area of the platinum foil is well defined and so, it serves as a good comparison for the BDD samples. BDD samples were prepared by the following steps:

1. The $\mathrm{SiO}_{2}$ layer on the surface without BDD was scratched off using a diamond scribe.

2. This was cleaned with dry air and/or methanol.

3. A small amount of indium/gallium eutectic was placed on the exposed $\mathrm{Si}$, where the $\mathrm{SiO}_{2}$ layer was scratched off. This forms ohmic contact with the sample.

4. A coiled (to increase surface area) wire was set in the indium/gallium eutectic.

5. A layer of silver paste was placed on the indium/gallium eutectic to keep the eutectic in place and secure the wire. The paste dries hard.

6. The silver paste was allowed to dry for a minimum of 30 minutes.

7. Epoxy, Hysol 1C, was then coated over the silver paste, back of the sample, and sample edges with the wire running through a glass tube. The epoxy also connected the sample with the glass tube. 
Figure A5(b) is BDD on a silicon substrate, Figure A5(c) is BDD on a tungsten substrate, and Figure A5(d) is BDD on a molybdenum substrate. The downfall of this method is that the electrode is permanently affixed with the epoxy and cannot be removed to be characterized after electrochemical testing. An epoxied electrode in an electrochemical cell is shown in Figure A6. The counter electrode is a platinum wire and the reference electrode $\mathrm{Ag} / \mathrm{AgCl}$.

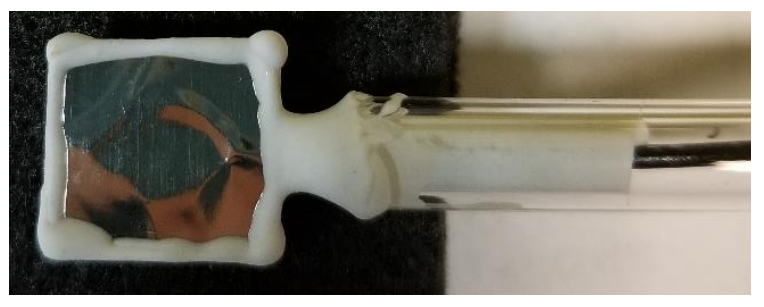

(a)

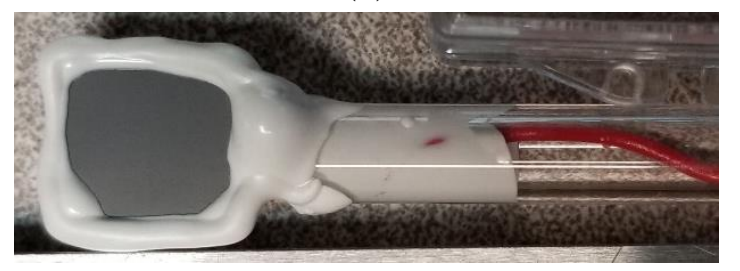

(c)

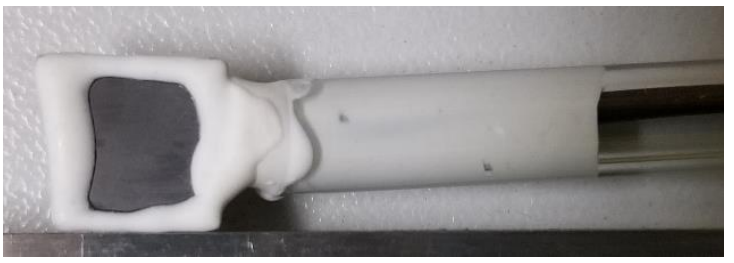

(b)

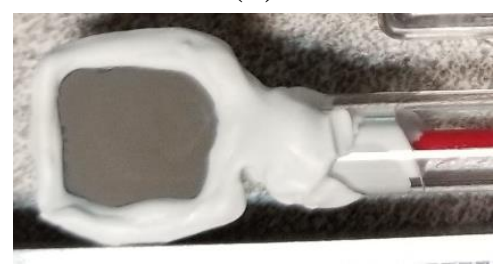

(d)

Figure A5. Electrodes encased in epoxy for three electrode testing (a) platinum foil, (b) BDD on a silicon substrate, (c) BDD on a tungsten substrate, and (d) BDD on a molybdenum substrate. 


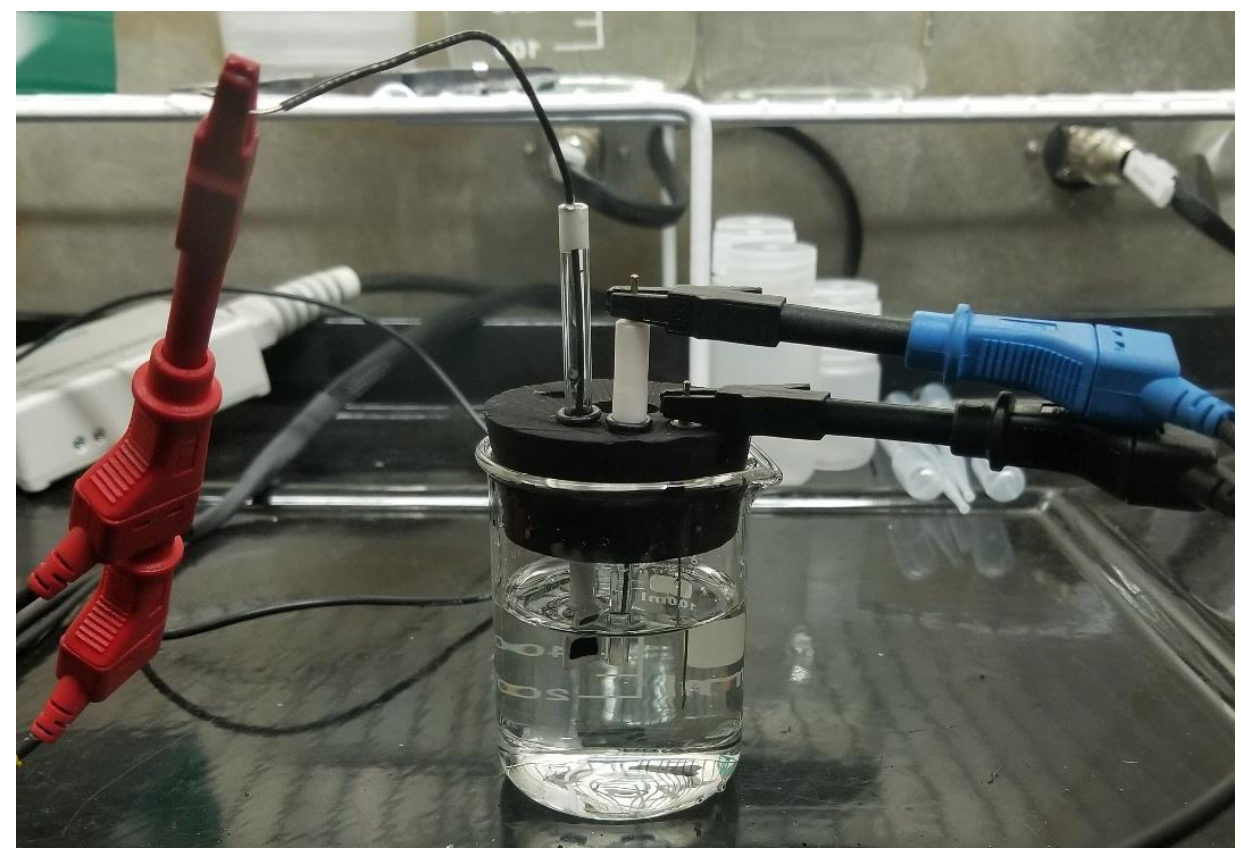

Figure A6. Three-electrode setup using a beaker and rubber stopper with a BDD working electrode, platinum wire counter electrode, and $\mathrm{Ag} / \mathrm{AgCl}$ reference electrode.

BDD grown on a metal substrate appeared well adhered before electrochemical testing. After electrochemical testing delamination was observed, shown in Figure A7.

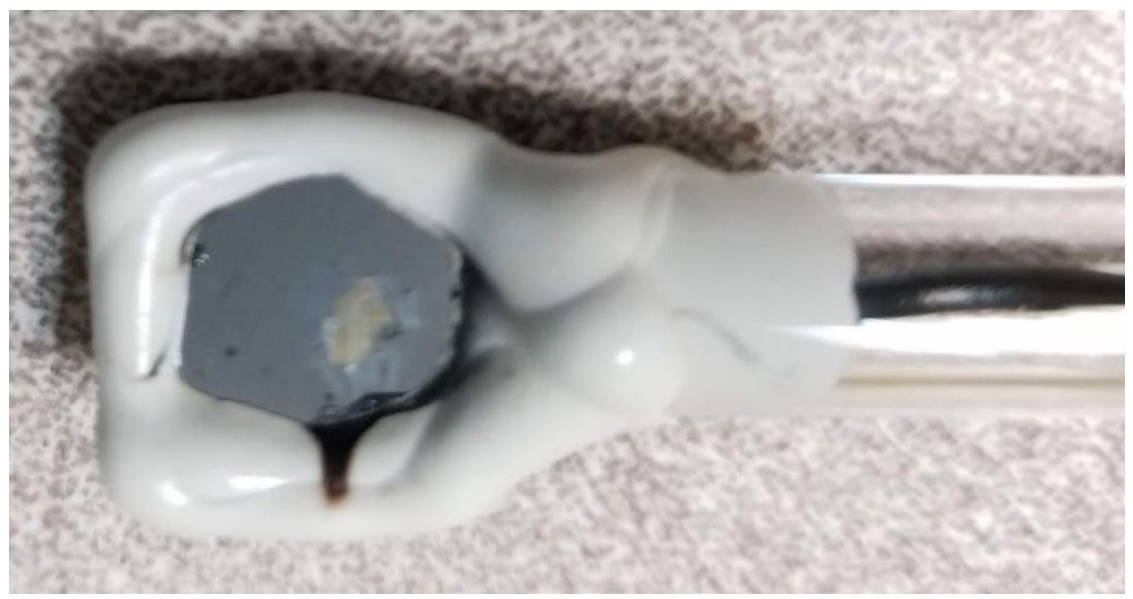

Figure A7. Delamination of BDD film on a tungsten substrate after extended use. 
BDD electrodes operated in different supporting electrolytes, $\mathrm{H}_{2} \mathrm{SO}_{4}$ and $\mathrm{HNO}_{3}$, were characterized using XPS and compared to an as-grown sample. This was done in an effort to observe the surface functionality of BDD. There was no significant different in the XPS spectra of these samples. Likely, any change in surface functionality corresponds with a change in a hydrogen group, which cannot be observed by XPS.

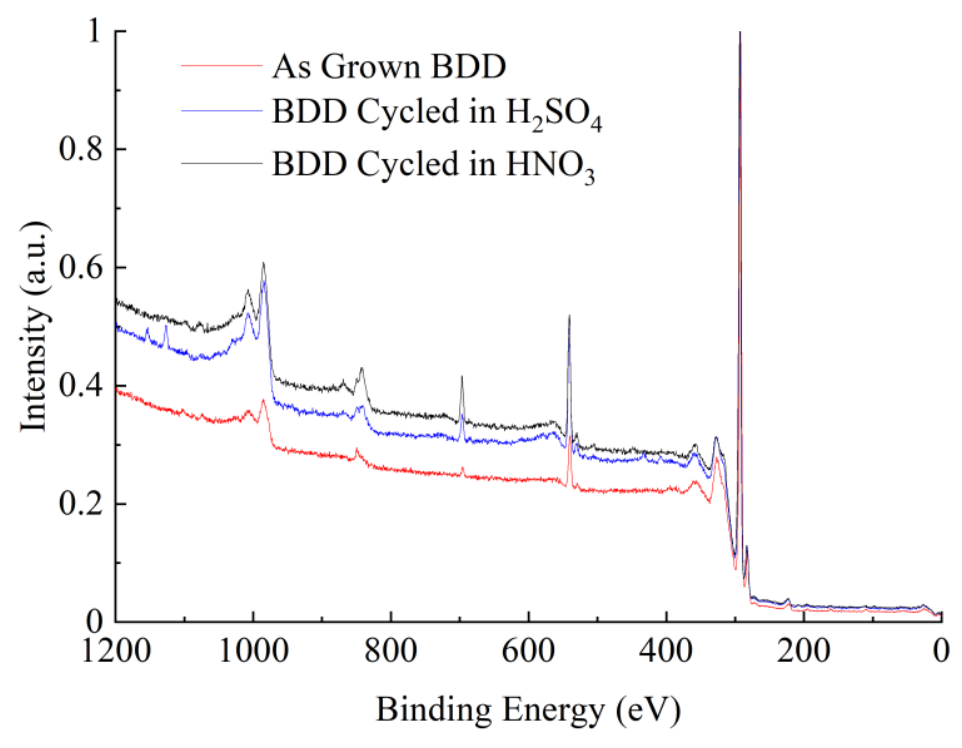

Figure A8. XPS spectra of an as-grown BDD sample, a BDD sample cycled in a sulfuric acid solution, and a BDD sample cycled in a nitric acid solution.

Nitrogen incorporated diamond was grown using the "Big Seki" reactor, shown in Figure A9. Nitrogen incorporated diamond is fundamentally different then BDD but, conductive, making it a potential electrode. These samples were tested in an electrochemical cell. Unfortunately, no reasonable data was obtained. 


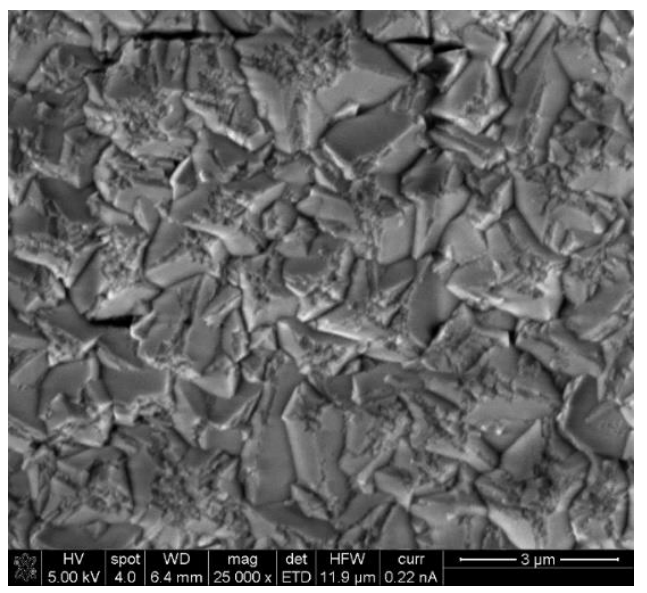

Figure A9. Nitrogen incorporated diamond grown on a silicon substrate.

Figure A10 shows the significant amount of $\mathrm{MnO}_{2}$ precipitation that occurs when using the $\mathrm{Mn}^{2+} / \mathrm{Mn}^{3+}$ redox couple. Initially this solution is clear, overtime it becomes cloudy, obtaining a rust like color. The precipitate coats the surface of the storage vessel and flow tubes. In Figure A10, there is no solution flowing, the color of the flow tubes is a result of the precipitate color alone.

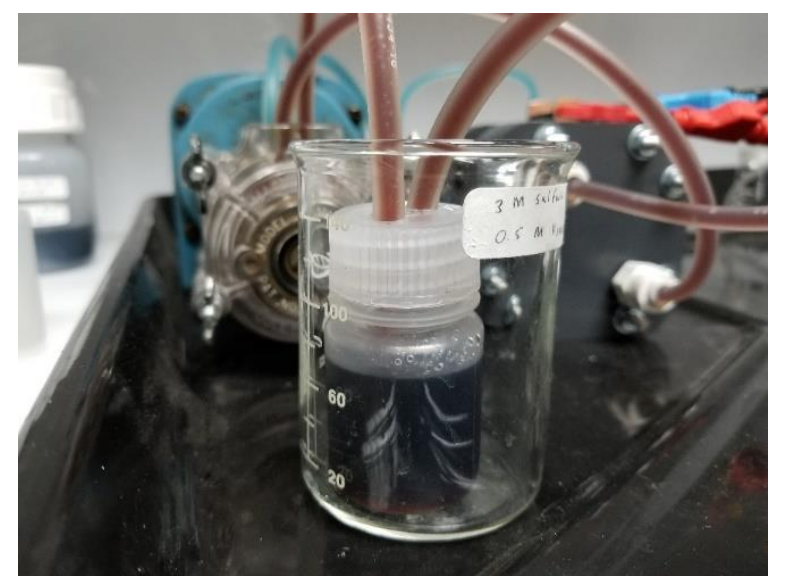

Figure A10. $\mathrm{MnO}_{2}$ precipitation coating tubes and turning electrolyte solution a rusty color. 
Redox couples not included in the main body of this manuscript due to their poor performance are shown in figure A11. Figure A11(a) is a BDD electrode with a Co active species electrolyte. The presence of the $\mathrm{Co}^{2+} / \mathrm{Co}^{3+}$ redox couple is observed; however, the overpotentials to the oxidation and reduction reactions are large. Figure A11(b) is a BDD electrode with a $\mathrm{Pb}$ active species electrolyte. The $\mathrm{Pb}^{2+} / \mathrm{Pb}^{4+}$ redox couple is observed. The overpotentials present are quite high and the CV curves are highly asymmetric, indicating the presence of side reactions. Figure A11(c) is a BDD electrode with a Ag active species electrolyte. This was in an attempt to obtain the $\mathrm{Ag}^{2+} / \mathrm{Ag}^{3+}$ redox couple. A reduction peak is observed but, no oxidation peak. The oxidation peak may be concealed by oxygen evolution. Figure 11(d) is BDD with a $\mathrm{Ti}$ active species. $\mathrm{The}^{\mathrm{TiO}}{ }_{2}{ }^{+} / \mathrm{Ti}^{3+}$ redox couple is observed. High overpotentials to the oxidation and reduction reactions are present along with overlap in hydrogen evolution. 


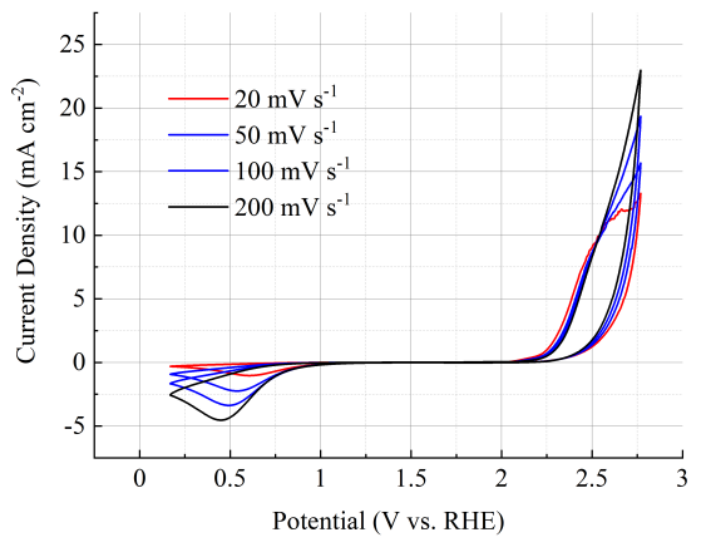

(a)

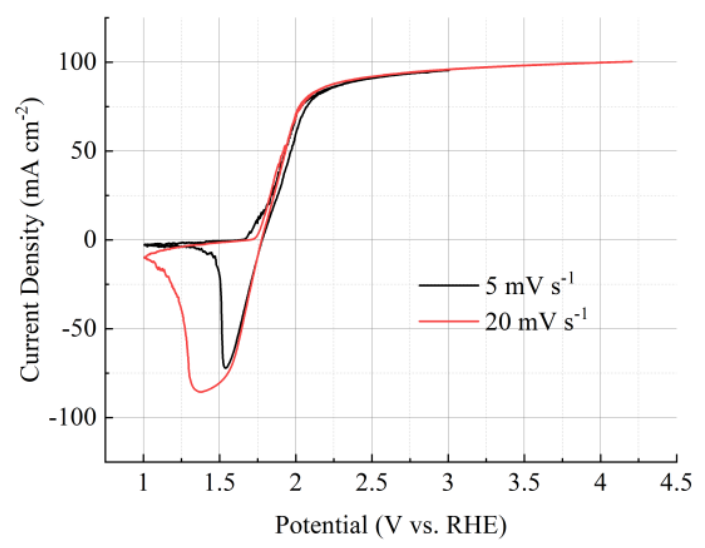

(c)

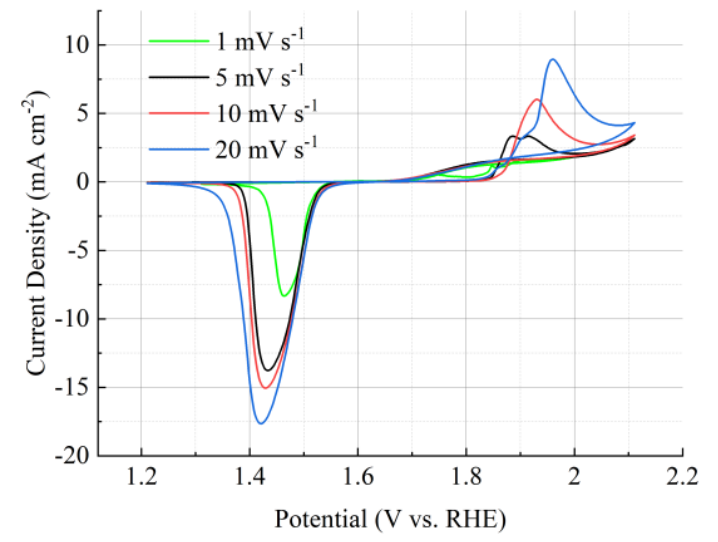

(b)

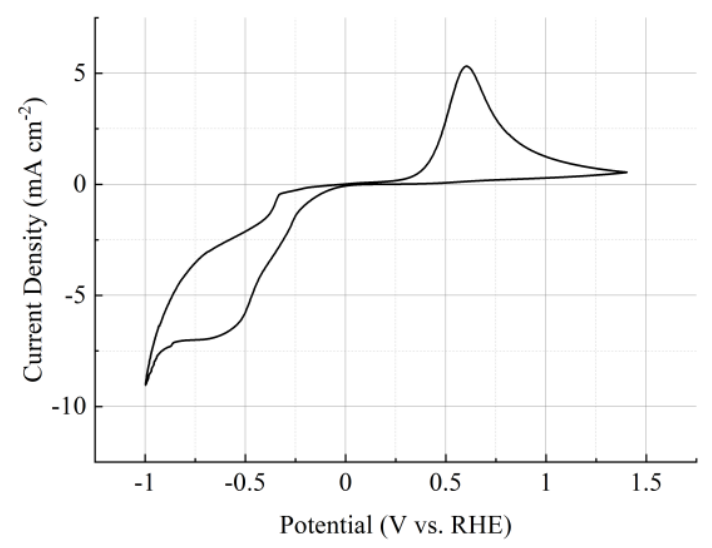

(d)

Figure A11. Alternative redox active species tested on a BDD electrode (a) $100 \mathrm{mM}$ $\mathrm{CoSO}_{4}$ in $5 \mathrm{M} \mathrm{H}_{2} \mathrm{SO}_{4}$, (b) $10 \mathrm{mM} \mathrm{Pb}\left(\mathrm{NO}_{3}\right)_{2}$ in $1 \mathrm{M} \mathrm{HNO}_{3}$, (c) $100 \mathrm{mM} \mathrm{AgNO} 3$ in $1 \mathrm{M}$ $\mathrm{HNO}_{3}$, and (d) $\mathrm{TiO}(\mathrm{SO})_{4}$ in $\mathrm{H}_{2} \mathrm{SO}_{4}$.

A plating reaction, $\mathrm{Zn} / \mathrm{Zn}^{2+}$, was attempted on the BDD surface, shown in Figure A12. After some time, a pellet of zinc formed. The zinc easily detached from the BDD surface, lending to the extreme fouling resistance of BDD. For a plating reaction, this is not desirable. In addition, the bottom of pellet was concave. It appears the pellet began growth around the edge of the Viton gasket. Had this not occurred, it is unlikely in solid deposition would adhere to the BDD surface. 


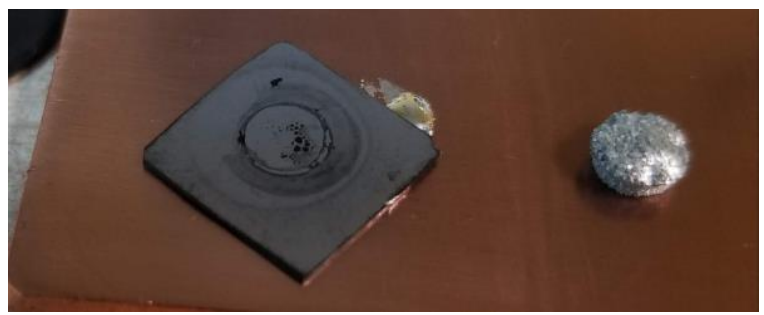

Figure A12. A zinc pellet formed as a result of electrodeposition on a BDD electrode.

Based on other research, it was thought a Lewis acid may catalyze some reactions on the BDD surface. Transition metal oxides tend to act as Lewis acids in an aqueous solution. So, many transition metal oxide powder suspensions were tested. Figure A13(a) shows the effect of $\mathrm{TiO}_{2}$ on the $\mathrm{Pb}^{2+} / \mathrm{Pb}^{4+}$ redox couple. The concentration of $\mathrm{TiO}_{2}$ appears to have an effect but, not in a beneficial way. Different concentrations of $\mathrm{TiO}_{2}$ was also tested with the $\mathrm{Mn}^{2+} / \mathrm{Mn}^{3+}$ redox couple on $\mathrm{BDD}$. $\mathrm{TiO}_{2}$ appears to be somewhat beneficial in reducing overpotential and increasing current density. This was expected to a degree, based on published literature. 


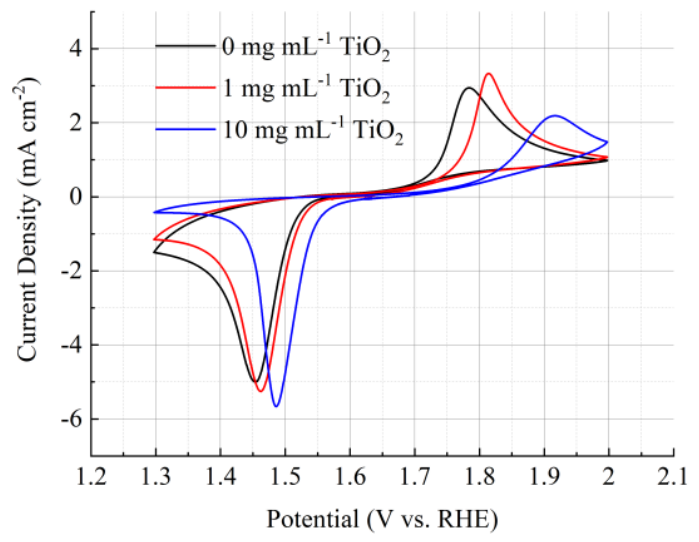

(a)

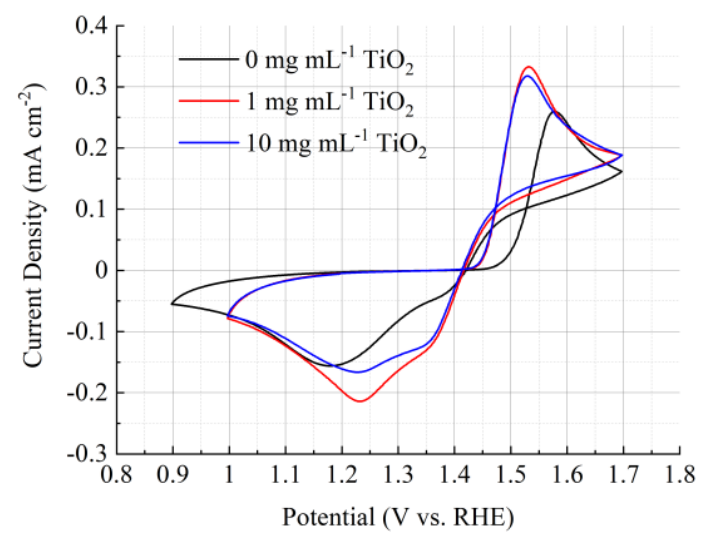

(b)

Figure A13. Increasing amounts of $\mathrm{TiO}_{2}$ as a potential catalyst in (a) $10 \mathrm{mM} \mathrm{Pb}\left(\mathrm{NO}_{3}\right)_{2}$ in $1 \mathrm{M} \mathrm{HNO}_{3}$ at $20 \mathrm{mV} \mathrm{s}^{-1}$, and (b) $10 \mathrm{mM} \mathrm{MnSO}_{4}$ in $1 \mathrm{M} \mathrm{H}_{2} \mathrm{SO}_{4}$ at $1 \mathrm{mV} \mathrm{s}^{-1}$.

The $\mathrm{Ce}^{3+} / \mathrm{Ce}^{4+}$ redox couple was observed to have potential in the main body of this manuscript. So, this redox couple was tested with various transition metal oxides in an effort to reduce overpotentials and/or increasing current density at the peak of oxidation and reduction. Figure A14(a) shows the $\mathrm{Ce}^{3+} / \mathrm{Ce}^{4+}$ redox couple with $\mathrm{TiO}_{2}$, which resulted in reduced performance. Figure A14(b) shows the $\mathrm{Ce}^{3+} / \mathrm{Ce}^{4+}$ redox couple with $\mathrm{SnO}_{2}$. There is no significant shift in performance. Figure A14(ac) shows the $\mathrm{Ce}^{3+} / \mathrm{Ce}^{4+}$ redox couple with $\mathrm{Al}_{2} \mathrm{O}_{3}$, resulting in reduced performance. Figure $\mathrm{A} 14(\mathrm{~d})$ shows the $\mathrm{Ce}^{3+} / \mathrm{Ce}^{4+}$ redox couple with $\mathrm{ZrO}_{2}$. There is no significant change in performance. 


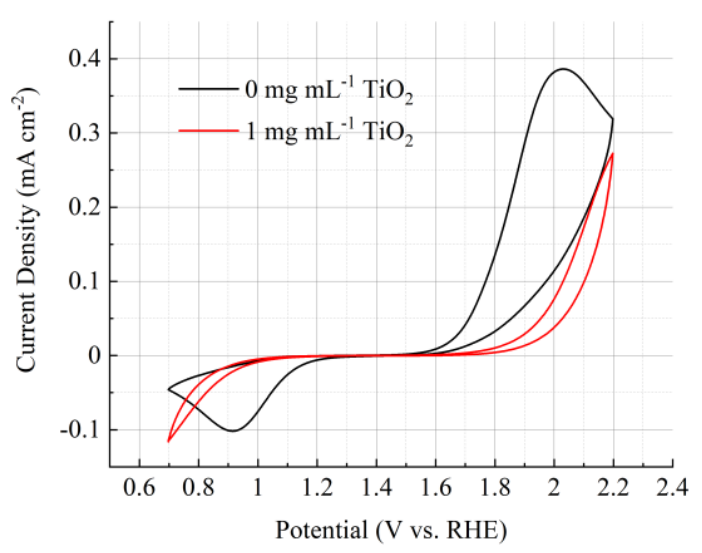

(a)

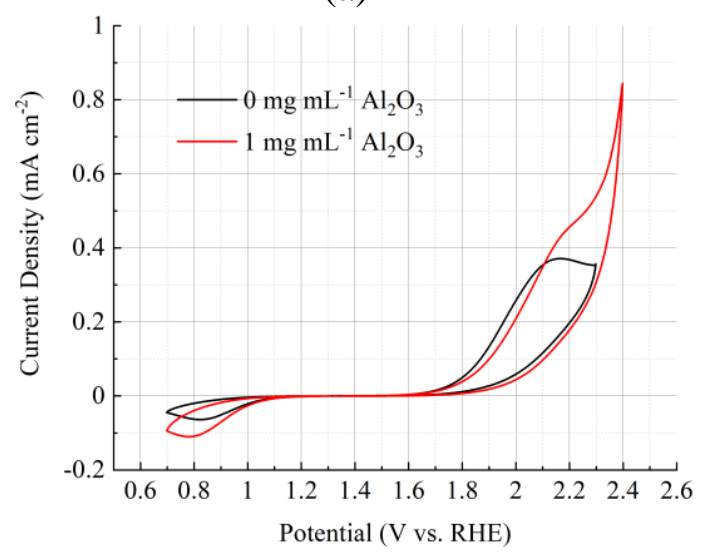

(c)

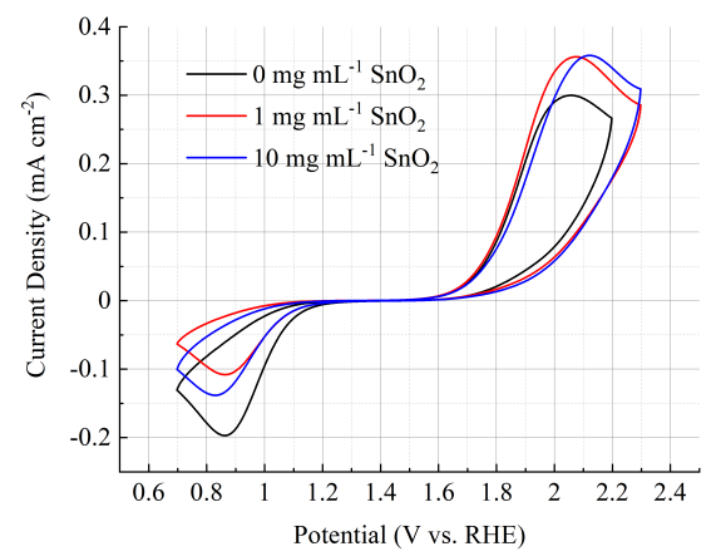

(b)

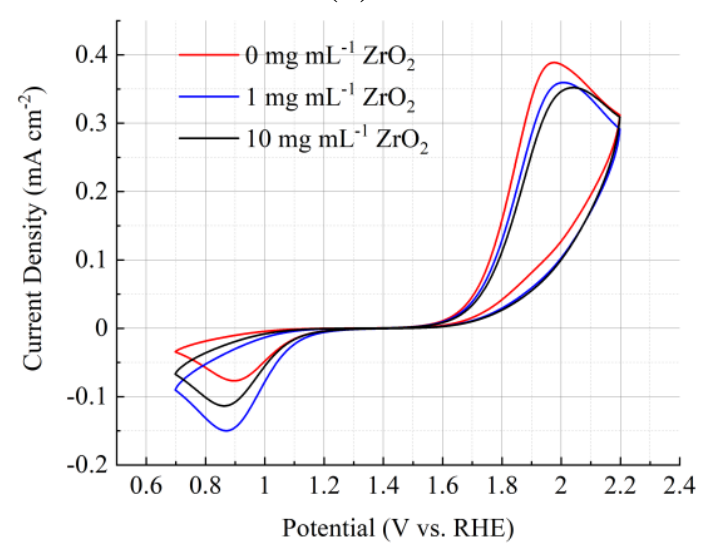

(d)

Figure A14. $10 \mathrm{mM} \mathrm{Ce}\left(\mathrm{NO}_{3}\right)_{3}$ in $1 \mathrm{M} \mathrm{H}_{2} \mathrm{SO}_{4}$ at $20 \mathrm{mV} \mathrm{s}^{-1}$ using increasing amounts of possible catalysts (a) $\mathrm{TiO}_{2}$, (b) $\mathrm{SnO}_{2}$, (c) $\mathrm{Al}_{2} \mathrm{O}_{3}$, and (d) $\mathrm{ZrO}_{2}$. 


\title{
CURRICULUM VITA
}

\author{
Alex M. Bates \\ 3611 Windward Way, Louisville, KY, 40209 \\ Cell: (502)888-4391・Email: alex.bates@louisville.edu
}

\section{Professional Profile}

Mechanical engineer with over 9 years of experience in fuel cell and redox flow battery design, fabrication, and testing, and 5 years of experience in microwave plasma-assisted chemical vapor deposition (MPCVD). My experience with MPCVD includes recipe modification, operation, troubleshooting, and reactor design for the growth of single and polycrystalline diamond, including boron doping and nitrogen incorporation, on various substrates. My core competencies include analyzing and interpreting experimental and characterization data to resolve issues, adjust experimental procedures, and discern future goals. I am a firm believer in continuous learning, teamwork, and the value of detail-oriented research.

\section{Education}

Doctor of Philosophy in Mechanical Engineering - GPA: 3.7/4.0

August 2020

University of Louisville, J.B. Speed School of Engineering Louisville, KY

Dissertation title: Analysis of Boron Doped Diamond as An Electrode for Aqueous Redox Flow Batteries

Master of Engineering in Mechanical Engineering - GPA: 3.6/4.0

University of Louisville, J.B. Speed School of Engineering

May 2015 Fuel Cell

Bachelor of Science in Mechanical Engineering - GPA: 3.7/4.0

University of Louisville, J.B. Speed School of Engineering

August 2014

Louisville, KY

\section{Experience}

PhD Student Researcher | University of Louisville

August 2015 - Present

- Identified a novel electrode material for utilization in redox flow batteries (RFBs), boron doped diamond (BDD)

- Demonstrated extreme corrosion resistance, high efficiency, and low fouling when using BDD as an electrode for RFBs with high potential redox couples including $\mathrm{Mn}^{2+} / \mathrm{Mn}^{3+}$ and $\mathrm{Ce}^{3+} / \mathrm{Ce}^{4+}$

- Designed a full cell RFB testing setup to demonstrate scaleup and optimization of BDD

- Lead the establishment, through purchasing and hands on setup, of a successful redox flow battery facility

$\circ \quad$ Designed and fabricated all components of an all-vanadium RFB with a greater than 80

$\%$ energy efficiency, meeting or exceeding current industry standards

- Developed a novel electrochemical cell for half-cell testing, which allows for complete before and after characterization of electrode materials

- Operated a microwave plasma-assisted chemical vapor deposition (MPCVD) reactor

- Grew and characterized BDD and nitrogen doped diamond samples on multiple substrates (silicon, molybdenum, graphite, etc.) with various growth recipes 
MEng Student Researcher | University of Louisville

August 2014 - August 2015

- Reduced fuel cell stack weight by more than $50 \%$ through the design of a novel flow plate

- Implemented novel flow plate designs into 3D simulation for flow field optimization while considering heat generation due to electrochemical reaction, heat transfer, and two-phase fluid flow including porous media

BSc Student Researcher | University of Louisville

May 2011 - August 2014

- Determined a method to optimize clamping pressure, which correlates with efficiency, in fuel cells through 3D simulation, validated experimentally

- Designed and simulated two different fuel cell flow plates which offer improved thermal distribution utilizing air cooling

Contract Engineer | Kentucky Advanced Materials Manufacturing Company

April 2020 - Present

- Comprehensive 3D modeling of a $30 \mathrm{~kW}$ microwave plasma-assisted chemical vapor deposition reactor designed to grow single crystal, gem quality diamond

- Disassembly and reassembly of an existing reactor system

- Cataloging all components, materials, surface finishes, etc.

Teaching Experience

University of Louisville | Mechanical Engineering

Summers 2016, 2018, 2020

Thermodynamics I and II (ME251 and ME310)

Louisville, KY

- Taught undergraduate courses of 50-70 students (official instructor)

University of Louisville | Mechanical Engineering

Graduate Teaching Assistant

May 2013 - April 2014

Louisville, KY

- Lectured and facilitated laboratory experiments for three required undergraduate mechanical engineering labs ( 120 students each semester) including Fluid Mechanics, Mechanics of Materials, and Mechanical Engineering

- Lead the development and fabrication of several undergraduate experiments

\section{Technical Skills}

Fabrication:

- Microwave plasma-assisted chemical vapor deposition (MPCVD)

- Diamond, boron doped diamond, nitrogen incorporated diamond on various substrates (silicon, molybdenum, tungsten, and graphite)

- Reactor design, modification, recipe identification, and troubleshooting

- Significant experience with Seki reactors, familiar with various other designs

- Computer aided design (CAD), computer numerical control (CNC) milling, and familiarity with 3D printing

\section{Characterization:}

- Electrochemical methods: Cyclic voltammetry (CV), Electrical impedance spectroscopy (EIS)

- Working knowledge with some experience: X-ray photoelectron spectroscopy (XPS), Scanning electron microscopy (SEM), Raman spectroscopy, Fourier-transform infrared spectroscopy (FTIR), Ultraviolet-visible spectroscopy (UV-vis)

FEA/CAD, Data Analysis, Electrochemistry:

- COMSOL Multiphysics, SolidWorks, HSMWorks,

- OriginPro, MS Office

- BioLogic EC-Lab, Autolab Nova 2.1, Gamry Framework, Arbin Console, Pine Research Aftermath

\section{Patents}

- Primary inventor on a provisional patent filed June 10, 2019, "Redox Flow Batteries Employing Diamond"

- Primary inventor on a patent purchased in South Korea, design of a low weight fuel cell flow plate 


\section{Selected Publications}

Total publications: 18, First author publications: 6, Total citations: 579

Bates, A. M., Paxton, W. F., Spurgeon, J. M., Park, S., Sunkara, M. K., Low-Cost Redox Flow Battery Chemistries Using Boron Doped Diamond Electrodes (In Preperation)

Bates, A. M., Paxton, W. F., Sunkara, M. K., Park, S., Three-dimensional Boron Doped Diamond Electrodes for Extreme Corrosion Resistance in High Potetnial Redox Flow Batteries (In Preperation)

Bates, A. M., Park, S., Recent Advances in Aqueous Redox Flow Battery Research (In Preperation)

Bates, A. M., Zickel, B., Krebs, S., Mukherjee, S., Schuppert, N. D., Choi, M., \& Park, S. (2017). Analytical Study and Experimental Validation of Copper II Sulfate and Potassium Ferri/Ferrocyanide Thermocells Using Onsager Flux Equations. Journal of Energy Resources Technology, 139(4). https://doi.org/10.1115/1.4036045

Bates, A., Mukherjee, S., Schuppert, N., Son, B., Kim, J. G., \& Park, S. (2015). Modeling and simulation of 2D lithium-ion solid state battery. International Journal of Energy Research, 39(11), 1505-1518. https://doi.org/10.1002/er.3344

Bates, A., Mukerjee, S., Lee, S. C., Lee, D., \& Park, S. (2014). An analytical study of a lead-acid flow battery as an energy storage system. Journal of Power Sources, 249, 207-218. https://doi.org/10.1016/j.jpowsour.2013.10.090

Bates, A., Hwang, S., Mukherjee, S., Lee, S. C., Kwon, O., Choi, G., \& Park, S. (2013). Simulation of an innovative polymer electrolyte membrane fuel cell design for self-control thermal management. International Journal of Hydrogen Energy, 38(20), 8422-8436. https://doi.org/10.1016/j.ijhydene.2013.04.114

Bates, A., Mukherjee, S., Hwang, S., Lee, S. C., Kwon, O., Choi, G., \& Park, S. (2013). Simulation and experimental analysis of the clamping pressure distribution in a PEM fuel cell stack. International Journal of Hydrogen Energy, 38(15), 6481-6493. https://doi.org/10.1016/j.ijhydene.2013.03.049

Bates, A., Mukherjee, S., Lee, S. C., Kwon, O., Ha, S., Thomas, S., Lee, D., Park, S. (2013). A High Energy Density System by Thin Metallic Bipolar Plates. ECS Transactions, 58(1), 223-227. http://doi.org/10.1149/05801.0223ecst

\section{Selected Presentations}

Enabling Efficient, Economical Grid-scale Energy Storage Through Diamond, 2019 MRS Fall Meeting \& Exhibit, Bates, A. M., Paxton, W. F., Spurgeon, J. M., Park, S., Sunkara, M. K.

Flow Batteries Using Manganese, Cerium and Related Redox Couples, 2019 MRS Fall Meeting \& Exhibit, Bates, A. M., Paxton, W. F., Spurgeon, J. M., Park, S., Sunkara, M. K.

\section{Honors and Awards}

- $\quad$ Four-year graduate fellowship recipient, University of Louisville, August 2015 - July 2019

- Hsing Chuang Award for Excellence in Graduate Study, University of Louisville, April 2015

- Mechanical Engineering Department Academic Achievement Awards, University of Louisville, April 2015 